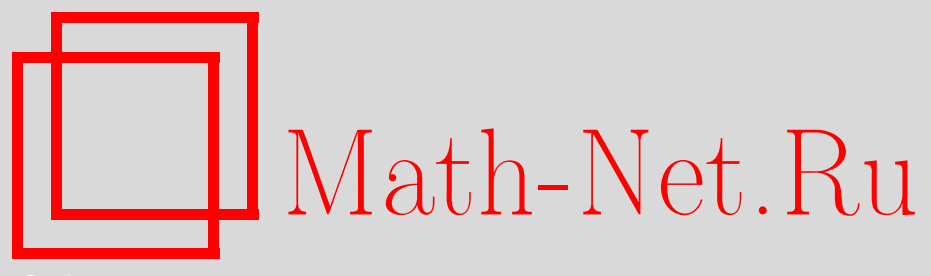

В. И. Богачев, Н. В. Крылов, М. Рёкнер, Эллиптические и параболические уравнения для мер, УМН, 2009, том 64, выпуск 6, 5-116

DOI: https://doi.org/10.4213/rm9326

Использование Общероссийского математического портала Math-Net.Ru подразумевает, что вы прочитали и согласны с пользовательским соглашением http://www . mathnet.ru/rus/agreement

Параметры загрузки:

IP : 18.209 .158 .208

26 апреля 2023 г., 18:06:26

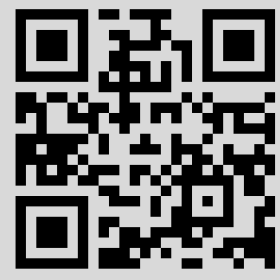




\section{Эллиптические и параболические уравнения для мер}

\section{В. И. Богачев, Н. В. Крылов, М. Рёкнер}

В статье дается обзор недавних исследований слабых эллиптических и параболических уравнений для мер с неограниченными и, возможно, сингулярными коэффициентами. Изучаются существование и дифференцируемость плотностей, обсуждаются нижние и верхние оценки для них. Исследуются полугруппы, связанные с эллиптическими операторами второго порядка в пространствах $L^{p}$ относительно инфинитезимально инвариантных мер.

Библиография: 181 название.

Ключевые слова: эллиптическое уравнение, параболическое уравнение, стационарное распределение диффузионного процесса, переходная вероятность.

\section{СОДЕРЖАНИЕ}

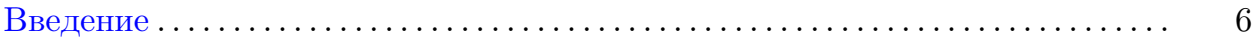

1. Инвариантные и инфинитезимально инвариантные меры .......... 10

1.1. Эллиптические уравнения для мер и существование плотностей 10

1.2. Локальные свойства решений ....................... 14

1.3. Некоторые априорные оценки.......................... 25

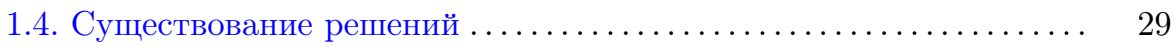

1.5. Ассоциированные $L^{1}$-полугруппы . . . . . . . . . . . . . . . . . . 32

1.6. О неединственности решений ........................ 43

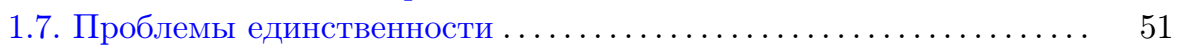

1.8. Глобальные свойства плотностей. . . . . . . . . . . . . . . . . . . 57

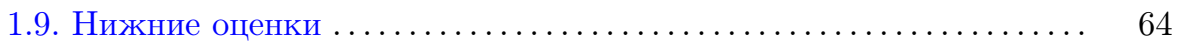

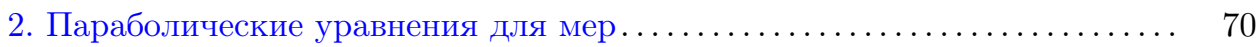

2.1. Априорные оценки . ............................... 70

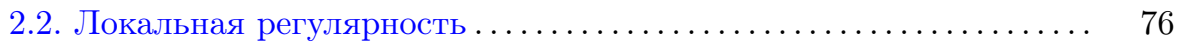

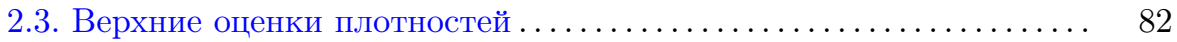

2.4. Неравенство Харнака для параболических уравнений ......... 86

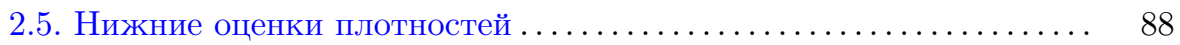

2.6. Уравнения Фоккера-Планка-Колмогорова ................ 93

Работа выполнена при поддержке РФФИ (гранты № 07-01-00536, 08-01-91205-ЯФ, 08-01-90431-Укр, 09-01-12180-офи-м) и программы SFB 701 при университете Билефельда.

(С) В. И. БоГАчев, Н. В. КРЫЛов, М. РЁкнеР, 2009 


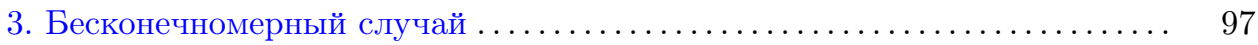

3.1. Уравнения для мер на бесконечномерных пространствах ...... 97

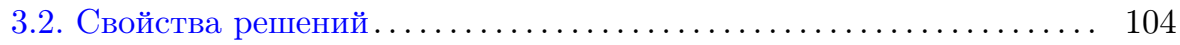

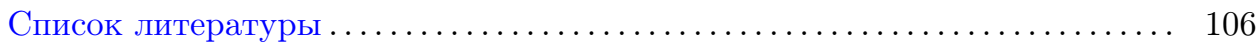

\section{Введение}

Цель этого обзора - систематическое изложение результатов, полученных за последние 10-15 лет для эллиптических и параболических уравнений для мер, типичными примерами которых являются уравнения Фоккера-Планка и Колмогорова. Это направление восходит к работам А. Н. Колмогорова [1], [2]. Одним из наших основных объектов является эллиптический оператор второго порядка

$$
L_{A, b} f=\operatorname{trace}\left(A f^{\prime \prime}\right)+(b, \nabla f), \quad f \in C_{0}^{\infty}(\Omega),
$$

где $A=\left(a^{i j}\right)$ - отображение на области $\Omega \subset \mathbb{R}^{d}$ со значениями в пространстве неотрицательных симметричных линейных операторов на $\mathbb{R}^{d}$ и $b=\left(b^{i}\right)-$ векторное поле на $\Omega$. В координатной форме $L_{A, b}$ задается выражением

$$
L_{A, b} f=a^{i j} \partial_{x_{i}} \partial_{x_{j}} f+b^{i} \partial_{x_{i}} f,
$$

где мы всегда подразумеваем суммирование по всем повторяющимся индексам.

С этим оператором $L_{A, b}$ мы связываем слабое эллиптическое уравнение

$$
L_{A, b}^{*} \mu=0
$$

для борелевских мер на $\Omega$, которое понимается в следующем слабом смысле:

$$
\int_{\Omega} L_{A, b} f d \mu=0 \quad \forall f \in C_{0}^{\infty}(\Omega),
$$

где предполагается, что $|b|, a^{i j} \in L_{\text {loc }}^{1}(\mu)$. Если мера $\mu$ имеет плотность $\varrho$, то $\varrho$ иногда называется "сопряженным решением", а уравнение называют "уравнением в двойном дивергентном виде".

Аналогично рассматриваются параболические операторы и параболические уравнения для мер на $(0, T) \times \mathbb{R}^{d}$.

Типичной чертой направления, обсуждаемого в этом обзоре, является то, что уравнение (1) имеет смысл при весьма широких предположениях относительно $A$ и $b$ : нужна лишь их локальная интегрируемость относительно решения $\mu$. Эти коэффициенты могут быть довольно сингулярны относительно меры Лебега, даже если решение допускает гладкую плотность. Например, для произвольной бесконечно дифференцируемой вероятностной плотности $\varrho$ на $\mathbb{R}^{d}$ мера $\mu=\varrho d x$ удовлетворяет уравнению (1) с $A=\mathrm{I}$ и $b=\nabla \varrho / \varrho$, где мы полагаем $\nabla \varrho(x) / \varrho(x)=0$ при $\varrho(x)=0$. Это очевидно из формулы интегрирования по частям

$$
\int_{\mathbb{R}^{d}}\left[\Delta f+\left\langle\frac{\nabla \varrho}{\varrho}, \nabla f\right\rangle\right] \varrho d x=\int_{\mathbb{R}^{d}} \varrho \Delta f d x+\int_{\mathbb{R}^{d}}\langle\nabla \varrho, \nabla f\rangle d x=0 .
$$

Поскольку $\varrho$ может обращаться в нуль на произвольном собственном замкнутом подмножестве $\mathbb{R}^{d}$, то векторное поле $b$ может быть локально не интегрируемым относительно меры Лебега, но оно локально интегрируемо относительно $\mu$. 
Отметим также, что в общем случае наши решения не могут быть более регулярными, чем коэффициенты (в отличие от случая обычных эллиптических уравнений). Например, если $d=1$ и $b=0$, то для произвольной положительной вероятностной плотности $\varrho$ мера $\mu=\varrho d x$ удовлетворяет уравнению $L_{A, 0}^{*} \mu=0$ с $A=\varrho^{-1} ;$ в частности, для гёльдерова $A$ наше решение может быть лишь гёльдеровым и не более того (а для разрывного $A$ может быть разрывным).

В этой общей постановке изучение слабых эллиптических уравнений для мер на конечномерных и бесконечномерных пространствах было предпринято в [3]-[12]. В действительности бесконечномерный случай был даже исходным пунктом, что мотивировалось исследованием бесконечномерных диффузионных процессов и другими применениями в бесконечномерном стохастическом анализе; например, близкие проблемы возникли в стохастическом квантовании в подходе, развитом А.И. Кирилловым [13]-[16], и в теории форм Дирихле (см. [17]). В ходе этих исследований было осознано, что даже для бесконечномерных уравнений с очень хорошими коэффициентами часто требуются результаты по конечномерным уравнениям с довольно общими коэффициентами. Например, мы увидим в п. 3.2, что конечномерные проекции $\mu_{n}$ меры $\mu$, удовлетворяющей эллиптическому уравнению на бесконечномерном пространстве, удовлетворяют эллиптическим уравнениям, коэффициенты которых представляют собой условные математические ожидания исходных коэффициентов относительно $\sigma$-алгебр, порожденных рассматриваемыми проектированиями. В результате даже для гладких бесконечномерных коэффициентов единственная информация об их условных математических ожиданиях связана с их интегрируемостью относительно $\mu_{n}$, а не относительно меры Лебега; в частности, не дано никакой локальной ограниченности. В настоящее время теория эллиптических и параболических уравнений для мер является быстро растущей областью с глубокими и интересными связями со многими направлениями в действительном анализе, теории уравнений с частными производными и стохастическом анализе.

Предположим, что $\xi_{t}$ - диффузионный процесс в $\mathbb{R}^{d}$, описываемый следующим стохастическим уравнением:

$$
d \xi_{t}=\sqrt{2 A\left(\xi_{t}\right)} d W_{t}+b\left(\xi_{t}\right) d t .
$$

Тогда всякая инвариантная мера $\mu$ процесса $\xi_{t}$ удовлетворяет $(1)$, а переходные вероятности процесса $\xi_{t}$ удовлетворяют соответствующему параболическому уравнению. Обратим внимание на то, что для диффузии, заданной стохастическим уравнением $d \xi_{t}=\sigma\left(\xi_{t}\right) d W_{t}+b\left(\xi_{t}\right) d t$, генератор переходной полугруппы имеет вид $L_{A, b}$, где $A=\sigma \sigma^{*} / 2$. Векторное поле $b$ называется коэфбициентом сноса или сносом. Матрицу $A=\left(a^{i j}\right)$ в операторе $L_{A, b}$ будем называть матрищей диффузии или коэффищиентом диффузии; это отличается от стандартной записи генератора диффузии отсутствием множителя $1 / 2$ при производных второго порядка.

Меры, удовлетворяющие (1), называются инфинитезимально инвариантнъмми, поскольку это уравнение имеет глубокие связи с инвариантностью относительно соответствующих операторных полугрупп.

Пусть $(X, \mathscr{B})$ - измеримое пространство и $B(X)$ - пространство всех ограниченных $\mathscr{B}$-измеримых функций на $X$, наделенное sup-нормой. Напомним, что 
если $\left(T_{t}\right)_{t \geqslant 0}$ - полугруппа ограниченных линейных операторов на пространстве $B(X)$, то ограниченная мера $\mu$ на $\mathscr{B}$ называется инвариантной для $\left(T_{t}\right)_{t \geqslant 0}$ (или $\left(T_{t}\right)_{t \geqslant 0}$-инвариантной), если

$$
\int_{X} T_{t} f d \mu=\int_{X} f d \mu \quad \forall f \in B(X) .
$$

Такие полугруппы естественно продолжаются на $L^{1}(\mu)$ и сильно непрерывны там во многих случаях, например, когда они задаются переходными вероятностями решений стохастических уравнений. Пусть $L$ - соответствующий генератор с областью определения $D(L)$. Тогда (3) равносильно равенству

$$
\int_{X} L f d \mu=0 \quad \forall f \in D(L) .
$$

При разумных предположениях относительно $A$ и $b$ генератор полугруппы, ассоциированной с диффузией, заданной указанным стохастическим уравнением, совпадает с $L_{A, b}$ на $C_{0}^{\infty}\left(\mathbb{R}^{d}\right)$. Как мы увидим, инвариантность меры в смысле $(3)$ - не то же самое, что (2). Дело в том, что класс $C_{0}^{\infty}\left(\mathbb{R}^{d}\right)$ может быть гораздо уже, чем $D(L)$. Важно то, что уравнение имеет смысл и может обладать решениями при предположениях, значительно более слабых, чем требуемые для существования диффузии, так что это уравнение может исследоваться без всяких предположений относительно существования полугрупп. С другой стороны, имеются очень интересные и плодотворные связи между обоими уравнениями; такие связи будут одной из центральных тем этой работы.

В двух первых разделах мы рассмотрим следующие проблемы на $\mathbb{R}^{d}$ (или, более общим образом, на конечномерных римановых многообразиях).

1) Регулярность решений уравнения (2), например, существование плотностей относительно меры Лебега, непрерывность и гладкость этих плотностей, а также некоторые связанные с ними оценки (такие как $L^{2}$-оценки для логарифмических градиентов решений). В частности, мы увидим в пп. 1.1, 1.2, что мера $\mu$ всегда абсолютно непрерывна на множестве $\{\operatorname{det} A>0\}$ и имеет непрерывную плотность из класса Соболева $W_{\text {loc }}^{p, 1}$ с $p>d$ при условии, что коэффициенты $a^{i j}$ входят в этот класс, $|b| \in L_{\text {loc }}^{p}(d x)$ или $|b| \in L_{\text {loc }}^{p}(\mu)$, причем матрица $A$ положительна. Условия на снос $|b| \in L_{\mathrm{loc}}^{p}(d x)$ или $|b| \in L_{\mathrm{loc}}^{p}(\mu)$ отнюдь не всегда равносильны. Например, первое из них приводит к положительности плотностей неотрицательных решений, а второе может быть выполнено и тогда, когда плотность имеет нули (случай сингулярного сноса); интересно, однако, что локальная $\mu$-интегрируемость $\exp (c|b|)$ уже обеспечивает положительность плотности. Глобальные свойства решений уравнений с неограниченными коэффициентами изучаются в пп. 1.8, 1.9, где получены некоторые глобальные верхние и нижние оценки для плотностей. Мы получим также аналогичные результаты для параболических уравнений; применительно к диффузионным процессам это означает регулярность переходных вероятностей. Результаты об априорной регулярности решений играют важную роль во многих других вопросах, например, о единственности и существовании решений. Поскольку нас 
интересуют сингулярные коэффициенты или коэффициенты низкой регулярности, мы совершенно не обсуждаем проблемы регулярности для вырожденных операторов с гладкими коэффициентами.

2) Существование решений эллиптического уравнения (2) и существование инвариантных мер в смысле (3), а также связи между обоими понятиями. В частности, мы увидим в пп. 1.4, 1.5, что при довольно общих предположениях для заданной вероятностной меры $\mu$, удовлетворяющей нашему эллиптическому уравнению (2), можно построить такую сильно непрерывную марковскую полугруппу $\left(T_{t}^{\mu}\right)_{t \geqslant 0}$ на $L^{1}(\mu)$, что $\mu$ является $\left(T_{t}^{\mu}\right)_{t \geqslant 0}$-инвариантной и генератор $\left(T_{t}^{\mu}\right)_{t \geqslant 0}$ совпадает с $L_{A, b}$ на $C_{0}^{\infty}\left(\mathbb{R}^{n}\right)$. Простое для проверки условие для этого - существование функции Ляпунова для $L_{A, b}$. В общем случае (без таких дополнительных предположений) верно немного меньше, а именно, $\mu$ лишь субинвариантна для $\left(T_{t}\right)_{t \geqslant 0}$. Мы увидим примеры, где такое действительно происходит, т. е. где $\mu$ не инвариантна.

3) Различные проблемы единственности; в частности, единственность инвариантных мер в смысле (3) и единственность решений уравнения (2) в классе всех вероятностных мер. Интересные родственные задачи касаются единственности ассоциированных полугрупп $\left(T_{t}^{\mu}\right)_{t \geqslant 0}$ и существенной самосопряженности оператора $L_{A, b}$ на $C_{0}^{\infty}\left(\mathbb{R}^{d}\right)$ в случае, когда он симметричен. Эти темы рассмотрены в пп. 1.5-1.7.

Сначала мы сконцентрируемся на эллиптическом случае, которому посвящен весь первый раздел. Во втором разделе похожие проблемы изучаются для параболических уравнений. Последний раздел посвящен краткому обсуждению бесконечномерных аналогов задач 1)-3). В отличие от конечномерного случая, где большинство представленных результатов получены в большой (иногда в максимальной) общности, не существует (и, возможно, не может существовать) никакой общей бесконечномерной теории. Результаты, полученные пока в бесконечномерном случае, применимы к различным частным ситуациям, но очень важно то, что они охватывают многие конкретные примеры, возникающие в приложениях, таких как стохастические дифференциальные уравнения с частными производными, бесконечночастичные системы, гиббсовские меры и т.д. Некоторые из конечномерных результатов неверны в бесконечной размерности, справедливость некоторых других (подходящим образом сформулированных) все еще неизвестна. Бесконечномерный случай будет предметом отдельного обзора; единственной целью последнего раздела было прокомментировать некоторое развитие бесконечномерных методов, результатов и техники.

В обзоре использованы как наши работы (включая совместные работы с рядом других авторов, в частности, [18]-[25]), так и работы многих других авторов, причем из-за ограниченности объема статьи в список литературы было включено менее четверти собранной нами библиографии. Ряд новых результатов приведен с доказательствами. Мы благодарны за полезные обсуждения С. Альбеверио, Ф. Ю. Вангу, А.Ю. Веретенникову, Дж. Да Прато, Б. Голдису, В.В. Жикову, Дж. Забчику, М. Закаю, Т.Ш. Зангу, А. И. Кириллову, В. А. Кондратьеву, Ю. Г. Кондратьеву, Г. Лехе, В. А. Лискевичу, П. Маллявэну, Дж. Метафуне, Д. Палларе, Э. Парду, А. Ранди, Г. Риттеру, Н. С. Трудингеру, С. В. Шапошникову, И. Шигекаве, В. Штаннату, А. Эберле, Д. Элворти. 


\section{1. Инвариантные и инфинитезимально инвариантные меры}

\section{1. Эллиптические уравнения для мер и существование плотно-} стей. Всюду ниже мы будем использовать следующие стандартные обозначения. Класс всех гладких функций с компактным носителем в открытом множестве $U \subset \mathbb{R}^{d}$ обозначается через $C_{0}^{\infty}(U)$. Класс всех ограниченных функций на $U$ с ограниченными производными всех порядков обозначается через $C_{b}^{\infty}(U)$; классы типа $C_{b}^{2}(U), C_{0}^{2}(U)$ и т. п. определяются аналогичным образом.

Термин “борелевская мера $\mu$ " будет обычно означать конечную (возможно, знакопеременную) счетноаддитивную меру на $\sigma$-алгебре борелевских множеств; случаи, когда рассматриваются бесконечные меры (скажем, локально конечные меры), будут всегда оговариваться за исключением меры Лебега. Интегрируемость функции относительно такой меры понимается как ее интегрируемость относительно полной вариации $|\mu|$ меры $\mu$; соответствующие классы будут обозначаться символами $L^{p}(\mu)$ или $L^{p}(\Omega, \mu)$ - в случае, когда $\mu$ рассматривается на фиксированном множестве $\Omega$. Символ $L^{p}(U)$ всегда указывает на меру Лебега; иногда с целью подчеркнуть это обстоятельство мы используем символ $L^{p}(U, d x)$. Как обычно, для $p \in[1,+\infty)$ положим $p^{\prime}:=p /(p-1)$.

Класс всех борелевских вероятностных мер на $\Omega$ будет обозначаться чеpeз $\mathscr{P}(\Omega)$.

Для заданного открытого множества $U \subset \mathbb{R}^{d}$ и $p \in[1,+\infty)$ обозначим через $W^{p, 1}(U)$ или $H^{p, 1}(U)$ класс Соболева функций $f \in L^{p}(U)$, у которых обобщенные частные производные $\partial_{x_{i}} f$ входят в $L^{p}(U)$. Это пространство наделено соболевской нормой

$$
\|f\|_{p, 1}:=\|f\|_{L^{p}}+\sum_{i=1}^{d}\left\|\partial_{x_{i}} f\right\|_{L^{p}} .
$$

Иногда мы используем также высшие классы Соболева $W^{p, k}(U)=H^{p, k}$ с $k \in \mathbb{N}$, состоящие из функций, у которых частные производные до порядка $k$ входят в $L^{p}(U)$, а также дробные пространства Соболева $H^{p, r}(U)$; обозначения с $H$ в основном будут использоваться в случае дробных или параболических классов Соболева. В немногих местах используются аналогично определяемые пространства Соболева $W^{p, 1}(\mu)$ относительно мер $\mu$ на $\mathbb{R}^{d}$ (в таких случаях мера $\mu$ имеет некоторые дополнительные свойства, например, обладает непрерывной положительной плотностью или слабо дифференцируемой плотностью, так что весовые классы Соболева корректно определены, см., например, [26]).

Символы типа $W_{\mathrm{loc}}^{p, 1}\left(\mathbb{R}^{d}\right), W_{\mathrm{loc}}^{p, 1}(U), L_{\mathrm{loc}}^{p}(U, \mu)$ и т. п. обозначают классы таких функций $f$, что $\zeta f$ входит в соответствующий класс без индекса loc для каждой функции $\zeta \in C_{0}^{\infty}(U)$.

В выражениях типа $a^{i j} b_{i}$ подразумевается стандартное правило суммирования по повторяющимся индексам. Скалярное произведение и норма в $\mathbb{R}^{d}$ обозначаются через $\langle\cdot, \cdot\rangle$ и $|\cdot|$ соответственно. Единичная матрица обозначается через I.

Предположим, что даны локально конечная борелевская (возможно, знакопеременная) мера $\mu$ на открытом множестве $\Omega \subset \mathbb{R}^{d}$, борелевское векторное поле $b$ на $\Omega$ и матричнозначное отображение $A=\left(a^{i j}\right)_{i, j \leqslant d}$ на $\Omega$ такие, что 
функции $a^{i j}$ измеримы по Борелю. Положим

$$
L_{A, b} \varphi:=\sum_{i, j \leqslant d} a^{i j} \partial_{x_{i}} \partial_{x_{j}} \varphi+\sum_{i \leqslant d} b^{i} \partial_{x_{i}} \varphi, \quad \varphi \in C_{0}^{\infty}(\Omega) .
$$

Для данной функции $c$ на $\Omega$ положим

$$
L_{A, b, c} \varphi=L_{A, b} \varphi+c \varphi .
$$

Мы будем рассматривать также операторы дивергентного вида

$$
\mathscr{L}_{A, b} \varphi:=\sum_{i, j \leqslant d} \partial_{x_{i}}\left(a^{i j} \partial_{x_{j}} \varphi\right)+\sum_{i \leqslant d} b^{i} \partial_{x_{i}} \varphi, \quad \varphi \in C_{0}^{\infty}(\Omega),
$$

и соответственно определенный оператор $\mathscr{L}_{A, b, c}$.

ОПРЕДЕЛЕНИЕ 1.1.1. Будем говорить, что мера $\mu$ удовлетворяет уравнению

$$
L_{A, b}^{*} \mu=0
$$

в $\Omega$, если $a^{i j}, b^{i} \in L_{\mathrm{loc}}^{1}(|\mu|)$, причем

$$
\int_{\Omega} L_{A, b} \varphi(x) \mu(d x)=0 \quad \forall \varphi \in C_{0}^{\infty}(\Omega) .
$$

Для заданной меры $\nu$ на $\Omega$ уравнение

$$
L_{A, b}^{*} \mu=\nu
$$

определяется аналогично. Наконец, для заданной функции $c \in L_{\text {loc }}^{1}(|\mu|)$ уравнение $L_{A, b, c}^{*} \mu=\nu$ определяется в таком же смысле.

Уравнение

$$
\mathscr{L}_{A, b}^{*} \mu=0
$$

определяется аналогично, но требует дополнительных предположений либо относительно $a^{i j}$, либо относительно $\mu$ (эти предположения будут делаться в подходящих местах).

Для фиксированной области $\Omega$ положим

$$
\mathscr{M}_{\mathrm{ell}}^{A, b}:=\left\{\mu \in \mathscr{P}(\Omega): L_{A, b}^{*} \mu=0\right\} .
$$

Ниже мы будем иметь дело со случаем, когда матрица $A$ симметрична и неотрицательна, но это не является необходимым для определений (в отличие от большинства результатов).

В общем случае уравнение (1.1.1) может не иметь ненулевых решений в классе ограниченных мер (возьмем $\Omega=\mathbb{R}^{1}, A=1, b=0$ ), может иметь много решений даже в классе вероятностных мер, а его решения могут быть довольно сингулярны (например, если $A=0$ и $b=0$, то всякая мера есть решение). Однако даже в рассмотренной общности имеется некоторая положительная информация.

Одномерный случай гораздо проще многомерного. 
ПреДЛОЖЕНИЕ 1.1.2. Пусть $d=1$ u $\Omega$ - интервал. Предположим, что $A>0$ на $\Omega$. Тогда всякая мера $\mu$, удовлетворяющая уравнению $L_{A, b, c}^{*} \mu=\nu$, абсолютно непрерывна относительно меры Лебега и имеет плотность $\varrho$ вида $\varrho=\varrho_{0} / A$, где $\varrho_{0}$ абсолютно непрерывна на каждом отрезке в $\Omega$.

Если $A=1, c=0, \nu=0, \Omega=(-1,1)$ и функиия $b$ локально интегрируема по Лебегу на $(-1,1)$, то

$$
\varrho(x)=\left(k_{1}+k_{2} \int_{0}^{x} \exp \left(-\int_{0}^{s} b(t) d t\right) d s\right) \exp \left(\int_{0}^{x} b(t) d t\right),
$$

где $k_{1}$ и $k_{2}$ - постояннье.

ДокАЗАТЕЛЬСТво. Мы имеем тождество

$$
\int_{\Omega}\left(A \varphi^{\prime \prime}+b \varphi^{\prime}+c \varphi\right) d \mu=\int_{\Omega} \varphi d \nu \quad \forall \varphi \in C_{0}^{\infty}(\Omega),
$$

которое можно записать как равенство

$$
(A \mu)^{\prime \prime}-(b \mu)^{\prime}+c \mu=\nu
$$

в смысле обобщенных функций. Значит, обобщенная производная от $(A \mu)^{\prime}-b \mu$ есть локально ограниченная мера. Это показывает, что обобщенная производная от $A \mu$ есть также локально ограниченная мера. Значит, мера $A \mu$ абсолютно непрерывна и имеет плотность $\varrho_{0}$. Следовательно, мера $\mu$ абсолютно непрерывна. Теперь из нашего рассуждения видно, что $(A \mu)^{\prime}-b \mu$ есть функция локально ограниченной вариации, значит, обобщенная производная от $A \mu$ есть локально интегрируемая функция, так что $\varrho_{0}$ допускает локально абсолютно непрерывную версию. В случае $A=1, c=0, \nu=0$ приходим к уравнению $\mu^{\prime \prime}-(b \mu)^{\prime}=0$, откуда $\mu^{\prime}-b \mu=k_{2}$ для некоторой постоянной $k_{2}$. Если функция $b$ локально интегрируема по Лебегу, то это уравнение явно решается. Предложение доказано.

Даже в этом простейшем одномерном случае мы замечаем, что решение $\mu$ может не иметь непрерывной плотности, если функция $A$ положительна, но не является непрерывной. На самом деле мы видим, что в случае невырожденного $A$ (т. е. $\operatorname{det} A \neq 0$ ) регулярность решения по существу такова, как регулярность $A$. Ниже мы увидим, что в высших размерностях картина похожа, хотя доказательства привлекают гораздо более тонкую технику. Другое простое наблюдение состоит в том, что без каких-либо предположений невырожденности $A$ мы получаем, что мера $A \cdot \mu$ абсолютно непрерывна. В высшей степени нетривиальный аналог этого справедлив также в многомерном случае.

Рассмотрим еще один поучительный пример.

ПримеР 1.1.3. Пусть $\varrho \in W_{\mathrm{loc}}^{1,1}\left(\mathbb{R}^{d}\right)$ и $\mu=\varrho d x$. Тогда $\mu$ удовлетворяет уравнению $L_{\mathrm{I}, b}^{*} \mu=0$, в котором

$$
b:=\frac{\nabla \varrho}{\varrho}, \quad \text { где } b(x):=0 \text { при } \varrho(x)=0 .
$$

В самом деле, функция $|b|$ локально $|\mu|$-интегрируема. Для всякого $\varphi \in C_{0}^{\infty}\left(\mathbb{R}^{d}\right)$ по формуле интегрирования по частям имеем

$$
\int[\Delta \varphi+\langle b, \nabla \varphi\rangle] \varrho d x=\int[-\langle\nabla \varphi, \nabla \varrho\rangle+\langle b, \nabla \varphi\rangle \varrho] d x=0,
$$


поскольку $b \varrho=\nabla \varrho$ почти всюду ввиду того факта, что $\nabla \varrho$ обращается в нуль почти всюду на множестве $\{\varrho=0\}$.

Отображение $\nabla \varrho / \varrho$ называется логарифмическим градиентом меры $\mu$ или плотности $\varrho$; оно определено, если $\varrho \in W_{\mathrm{loc}}^{1,1}\left(\mathbb{R}^{d}\right)$.

В этом примере можно взять $\varrho$ даже бесконечно дифференцируемой, но $b$ может быть довольно сингулярным относительно меры Лебега. Например, для заданного собственного замкнутого подмножества $Z \subset \mathbb{R}^{d}$ найдется вероятностная плотность $\varrho \in C^{\infty}\left(\mathbb{R}^{d}\right)$ с $Z=\{\varrho=0\}$; таким способом можно даже получить $b$, не являющееся локально интегрируемым по Лебегу на замкнутом множестве положительной меры Лебега. Простейший пример сингулярности таков:

$$
\varrho(x)=\frac{x^{2} \exp \left(-x^{2} / 2\right)}{\sqrt{2 \pi}}, \quad b(x)=x+\frac{2}{x} .
$$

Теперь сформулируем основные результаты о существовании плотностей; доказательства см. в [9].

Теорема 1.1.4. Предположим, что матрицы $A(x)$ симметричны и неотрицательны. Пусть $\mu$ - локально конечная борелевская мера на $\Omega$, причем $a^{i j} \in L_{\mathrm{loc}}^{1}(\Omega, \mu)$ и для некоторого $C>0$ имеем

$$
\int_{\Omega} a^{i j} \partial_{i} \partial_{j} \varphi d \mu \leqslant C\left(\sup _{\Omega}|\varphi|+\sup _{\Omega}|\nabla \varphi|\right)
$$

для всех неотрицательных $\varphi \in C_{0}^{\infty}(\Omega)$. Тогда справедливы следуюшие утверждения.

(i) Если н неотрицательна, то $(\operatorname{det} A)^{1 / d} \mu$ имеет плотность в $L_{\text {loc }}^{d^{\prime}}(\Omega, d x)$.

(ii) Если $A$ локально гёльдерово $u \operatorname{det} A>0$, то $\mu$ имеет плотность, которая входит в $L_{\mathrm{loc}}^{r}(\Omega, d x)$ для каждого $r \in\left[1, d^{\prime}\right)$.

Мы не знаем, остается ли верным утверждение (i) для знакопеременных мер.

СлеДСТвИЕ 1.1.5. Пусть $\mu$ - локально конечная знакопеременная борелевская мера на $\Omega$ и $a^{i j}, b^{i}, c \in L_{\mathrm{loc}}^{1}(\Omega, \mu)$. Предположим, что

$$
\int_{\Omega}\left(L_{A, b} \varphi+c \varphi\right) d \mu \leqslant 0 \quad \text { для всех неотрицательнъх } \varphi \in C_{0}^{\infty}(\Omega) .
$$

Тогда справедливы следующие утверждения.

(i) Если мера $\mu$ неотрицательна, то $(\operatorname{det} A)^{1 / d} \mu$ имеет плотность из класca $L_{\text {loc }}^{d^{\prime}}(\Omega, d x)$.

(ii) Если $A$ локально гёльдерово $u \operatorname{det} A>0$, то $\mu$ имеет плотность, которая входит в $L_{\mathrm{loc}}^{r}(\Omega, d x)$ для каждого $r \in\left[1, d^{\prime}\right)$.

В частности, эти утверждения верны, если выполнено (1.1.1).

В утверждении (ii) этого следствия нельзя ожидать, что плотность $\mu$ будет гёльдерова, поскольку для $d=1$ и $A=1$ можно взять

$$
\mu(d x)=\exp \left(\int_{0}^{x} b(t) d t\right) d x
$$

с подходящей функцией $b$.

Предыдущее следствие имеет следующее важное обобщение. 
СлЕДСТвИЕ 1.1.6. Пусть $\mu$ и -две локально конечные знакопеременные борелевские меры на $\Omega$ и $a^{i j}, b^{i}, c \in L_{\mathrm{loc}}^{1}(\Omega, \mu)$. Предположим, что

$$
\int_{\Omega}\left[L_{A, b} \varphi+c \varphi\right] d \mu=\int_{\Omega} \varphi d \nu \quad \text { для всех неотрицателъных } \varphi \in C_{0}^{\infty}(\Omega) .
$$

Тогда справедливы следующие утверждения.

(i) Если мера $\mu$ неотрицательна, то $(\operatorname{det} A)^{1 / d} \mu$ имеет плотность из класca $L_{\text {loc }}^{d^{\prime}}(\Omega, d x)$.

(ii) Если $A$ локально гёлъдерово $u \operatorname{det} A>0$, то $\mu$ имеет плотность, которая входит в $L_{\mathrm{loc}}^{r}(\Omega, d x)$ для каждого $r \in\left[1, d^{\prime}\right)$.

ЗАмЕчАниЕ 1.1.7. (i) Утверждение (i) теоремы 1.1.4, следствие 1.1.5 и следствие 1.1.6 для неотрицательных мер распространяются на случай, когда $\mu$ является $\sigma$-конечной неотрицательной борелевской мерой на $\Omega$ (не обязательно локально ограниченной). Действительно, (1.1.3), (1.1.4) и (1.1.5) имеют смысл также для $\sigma$-конечной меры $\mu$ при условии, что $a^{i j}, b^{i}, c \in L_{\mathrm{loc}}^{1}(\Omega, \mu)$. Найдем такую вероятностную меру $\mu_{0}$, что $\mu=f \mu_{0}$, где $f$ - положительная борелевская функция. Пусть

$$
a_{0}^{i j}:=f a^{i j}, \quad b_{0}^{i}:=f b^{i}, \quad c_{0}:=f c, \quad A_{0}=\left(a_{0}^{i j}\right)_{i, j \leqslant d}, \quad b_{0}=\left(b_{0}^{i}\right)_{i \leqslant d} .
$$

Ясно, что $a_{0}^{i j}, b_{0}^{i}, c_{0} \in L_{\text {loc }}^{1}\left(\mu_{0}\right)$ и $\mu_{0}$ удовлетворяет предположениям упомянутых выше утверждений с $A_{0}, b_{0}$ и $c_{0}$ вместо $A, b$ и $c$. Значит, мера $\left(\operatorname{det} A_{0}\right)^{1 / d} \mu_{0}$ имеет плотность $\varrho \in L_{\text {loc }}^{d^{\prime}}(\Omega, d x)$. Поскольку $\left(\operatorname{det} A_{0}\right)^{1 / d}=f(\operatorname{det} A)^{1 / d}$, это означает, что $(\operatorname{det} A)^{1 / d} \mu$ имеет ту же самую плотность.

(ii) Пусть выполнены условия следствия 1.1 .5 (ii) и $B_{R_{1}}\left(x_{0}\right)$ - шар в $\Omega$ радиуса $R_{1}>0$ с центром в некотором $x_{0}$. Тогда для всяких $R<R_{1}$ и $r<d^{\prime}$ существует такое $N$, зависящее только от $R_{1}, R, r, d, \inf _{B_{R_{1}}} \operatorname{det} A, \sup _{i, j} \sup _{B_{R_{1}}}\left|a^{i j}\right|$ и гёльдеровой нормы $A$ на $B_{R_{1}}$, что для плотности $\varrho$ меры $\mu$ имеем

$$
\|\varrho\|_{L^{r}\left(B_{R}\right)} \leqslant N\|1+|b|+|c|\|_{L^{1}\left(B_{R_{1}}, \mu\right)} .
$$

Кроме того, при фиксированном $d$ число $N$ является локально ограниченной функцией от указанных выше величин. Это вытекает из доказательства теоремы 1.1.4 в [9].

1.2. Локальные свойства решений. Перейдем к результатам о регулярности решений. До конца этого пункта будем предполагать, что матрицы $A(x)$ симметричны и положительны и $A(x)$ непрерывно по $x$. По теореме вложения Соболева условие непрерывности заведомо выполнено для некоторой версии $A$, если $a^{i j} \in W_{\text {loc }}^{p, 1}$, где $p>d$. Мы не обсуждаем здесь случая гладких коэффициентов и, возможно, вырожденного $A$ при условии Хёрмандера и его аналогов в исчислении Маллявэна (см. ссылки в [26]).

Прежде всего напомним один классический результат.

Теорема 1.2.1. Предположим, что функции $a^{i j}, b^{i}$, с бесконечно дифберенцируемы $u \operatorname{det} A>0$ в $\Omega$. Если $\nu$ имеет бесконечно дифберенцируемую плотность, то всякое решение уравнения $L_{A, b, c}^{*} \mu=\nu$ обладает бесконечно дифберениируемой плотностъю.

Далее, рассмотрим случай, когда коэффициенты лишь гёльдеровы. Следующий результат доказан в [27]. 
ТеОРема 1.2.2. Предположим, что коэффициенты $a^{i j}, b^{i}$, с локально гёльдеровы в $\Omega$ u $\operatorname{det} A>0$. Тогда всякое решение $\mu$ уравнения $L_{A, b, c}^{*} \mu=0$ имеет локально гёльдерову плотность.

Заметим, что решения в [27] были по предположению локально интегрируемыми функциями, но ввиду приведенных выше результатов теорема остается справедливой и для мер. Было бы интересно изучить случай, когда лишь коэффициенты $a^{i j}$ гёльдеровы. Непрерывность всех коэффициентов не гарантирует гёльдеровость решения даже при $d=1$ и $A>0$. Однако неясно, непрерывны ли плотности решений в случае, когда коэффициенты лишь непрерывны и $A$ невырождено.

Перейдем к наиболее трудному случаю, когда коэффициент диффузии несколько лучше, чем гёльдеров, но не является гладким, а мы желаем иметь некоторую соболевскую регулярность плотности решений. Одна из причин, почему это важно, состоит в том, что, установив соболевскую регулярность наших решений, мы можем переписать уравнение $L_{A, b, c}^{*} \mu=0$ для меры $\mu$ как классическое уравнение на ее плотность $\varrho$ в смысле слабых решений: действительно, интегрируя по частям, находим

$$
\int_{\Omega}\left[a^{i j} \partial_{x_{i}} \varrho \partial_{x_{j}} \varphi+\partial_{x_{i}} a^{i j} \partial_{x_{j}} \varphi \varrho+b^{i} \partial_{x_{i}} \varphi \varrho+c \varrho\right] d x=0
$$

для всех $\varphi \in C_{0}^{\infty}(\Omega)$.

Tеорема 1.2.3. Пусть $d \geqslant 2, p \geqslant d, q \in(1, \infty), R_{1}>0, a^{i j} \in W^{p, 1}\left(B_{R_{1}}\right)$ и $A \geqslant \lambda \mathrm{I}$, где $\lambda>0$. Тогда найдутся числа $R_{0}>0$ и $N_{0}>0$ со следуюшими свойствами. Пусть $R<R_{0}$ и мера $\mu$ ограниченной вариачии на $B_{R}$ такова, что для всякого $\varphi \in C_{0}^{2}\left(B_{R}\right):=C^{2}\left(\overline{B_{R}}\right) \cap\left\{u:\left.u\right|_{\partial B_{R}}=0\right\}$ мы имеем

$$
\left|\int_{B_{R}} a^{i j} \partial_{i} \partial_{j} \varphi d \mu\right| \leqslant N\|\nabla \varphi\|_{L^{q}\left(B_{R}\right)},
$$

где $N$ не зависит от $\varphi$. Предположим далее, что выполнено одно из следующих условии:

a) $p>d u л u$

b) $p=d>q^{\prime} u \mu \in \bigcup_{r>1} L^{r}\left(B_{R}\right)$.

Тогда $\mu \in W_{0}^{q^{\prime} \wedge p, 1}\left(B_{R}\right)$ (где мъ отождествляем $\mu$ с ее плотностъю) $u$

$$
\|\mu\|_{W_{0}^{q^{\prime} \wedge p, 1}\left(B_{R}\right)} \leqslant N_{0} .
$$

Кроме того, радиус $R_{0}$ зависит толъко от $p, q, d, \lambda, R_{1},\left\|a^{i j}\right\|_{W^{p, 1}\left(B_{R_{1}}\right)}$ и скорости убывания $\left\|\nabla a^{i j}\right\|_{L^{d}\left(B_{R}\right)}$ при $R \rightarrow 0$, а число $N_{0}$ зависит от тех же величин и $N$.

ЗАмечАниЕ 1.2.4. Доказательство этой теоремы фактически показывает, что если $\mu$ имеет компактный носитель в $B_{R_{1}}$ и (1.2.1) выполнено для всех $\varphi \in C_{0}^{\infty}\left(B_{R_{1}}\right)$, то $\mu \in W_{0}^{q^{\prime} \wedge p, 1}\left(B_{R}\right)$ для некоторого $R<R_{1}$. Более того, даже без предположения о компактности носителя можно показать, что $\mu \in$ $W_{\text {loc }}^{q^{\prime} \wedge p, 1}\left(B_{R}\right)$, но это требует некоторой дополнительной работы.

Эта теорема сразу дает некоторую низкую регулярность решений эллиптических уравнений. 
СледСтвие 1.2.5. Предположим, что $p>d \geqslant 2, a^{i j} \in W_{\mathrm{loc}}^{p, 1}(\Omega), \operatorname{det} A>0$ и н удовлетворяет уравнению $L_{A, b}^{*} \mu=0$, где $b \in L_{\mathrm{loc}}^{r}(\mu)$ для некоторого $r>1$. Тогда $\mu$ имеет плотность из класса $W_{\mathrm{loc}}^{\alpha, 1}(\Omega)$ для каждого $\alpha<d r /(d r-r+1)$.

Можно получить больше, если $b$ лучше интегрируемо.

TEOPEMA 1.2.6. Пусть $p>d, r \in\left(p^{\prime}, \infty\right), \mu=\varrho d x, \varrho \in L_{\mathrm{loc}}^{r}(\Omega, d x)$, $a^{i j} \in W_{\mathrm{loc}}^{p, 1}(\Omega)$, и пусть либо $\beta \in L_{\mathrm{loc}}^{p}(\Omega, d x)$, либо $\beta \in L_{\mathrm{loc}}^{p}(\Omega, \mu)$. Пусть $A^{-1}$ локально равномерно ограничено. Предположим, что для каждого $\varphi \in C_{0}^{\infty}(\Omega)$ мы имеем

$$
\left|\int_{\Omega} a^{i j} \partial_{i} \partial_{j} \varphi \mu(d x)\right| \leqslant \int_{\Omega}(|\varphi|+|\nabla \varphi|)|\beta||\mu|(d x) .
$$

Тогда $\varrho \in W_{\mathrm{loc}}^{p, 1}(\Omega)$.

ЗАмечАниЕ 1.2.7. Следует отметить, что заключение теоремы 1.2 .6 справедливо при следующих альтернативных предположениях относительно $a^{i j}$, $\beta, \mu: \mu$ - локально ограниченная борелевская мера на $\Omega$ (без условий на плотность), отображение $A^{-1}$ локально равномерно ограничено, $\beta \in L_{\mathrm{loc}}^{p}(\Omega, \mu)$ или $\beta \in L_{\mathrm{loc}}^{1}(\Omega, \mu) \cap L_{\mathrm{loc}}^{p}(\Omega, d x)$. Это вытекает из теоремы 1.1.4.

СлЕДСТВИЕ 1.2.8. Пусть $\mu$ - локально конечная борелевская мера на $\Omega$ u $A^{-1}$ локально равномерно ограничено в $\Omega$, причем $a^{i j} \in W_{\mathrm{loc}}^{p, 1}(\Omega)$, где $p>d$, и пусть либо (i) $b^{i}, c \in L_{\mathrm{loc}}^{p}(\Omega, d x)$, либо (ii) $b^{i}, c \in L_{\mathrm{loc}}^{p}(\Omega, \mu)$. Предположим, что для каждого $\varphi \in C_{0}^{\infty}(\Omega)$ мъи имеем

$$
\int_{\Omega}\left[a^{i j} \partial_{i} \partial_{j} \varphi+b^{i} \partial_{i} \varphi+c \varphi\right] d \mu=0
$$

где в случае (i) дополнительно предполагается, что функиии $b^{i}$, с локально $\mu$-интегрируемы. Тогда $\mu$ имеет плотность из $W_{\mathrm{loc}}^{p, 1}(\Omega)$, которая локально гёльдерова.

Следующий результат, доказанный в [23], является полезной модификацией предыдущей теоремы.

TеОРема 1.2.9. Пусть $p>d, r \in\left(p^{\prime}, \infty\right)$ и $\mu$-мера на $B_{R}$ с плотностью $\varrho \in L_{\mathrm{loc}}^{r}\left(B_{R}\right)$. Пyсть $a^{i j} \in W_{\mathrm{loc}}^{p, 1}\left(B_{R}\right), \beta_{1} \in L_{\mathrm{loc}}^{p}\left(B_{R}\right), \beta_{2} \in L_{\mathrm{loc}}^{p}(\mu)$, где $A^{-1}$ локально равномерно ограничено на $B_{R}$. Предположим, что для каждого $\varphi \in$ $C_{0}^{\infty}\left(B_{R}\right)$ миц имеем

$$
\left|\int_{B_{R}} a^{i j} \partial_{x_{i}} \partial_{x_{j}} \varphi d \mu\right| \leqslant \int_{B_{R}}\left(|\varphi|+\left|\nabla_{x} \varphi\right|\right)\left(\left|\beta_{1} \varrho\right|+\left|\beta_{2} \varrho\right|\right) d x .
$$

Тогда $\varrho \in W_{\mathrm{loc}}^{p, 1}\left(B_{R}\right)$, а значит, $\varrho$ обладает локалъно гёлъдеровой версией.

СлеДСтвиЕ 1.2.10. Пусть $\mu$ - локально конечная борелевская мера на $B_{R}$, $A^{-1}$ локально ограничено на $B_{R}, a^{i j} \in W_{\mathrm{loc}}^{p, 1}\left(B_{R}\right)$, где $p>d, \partial_{x_{i}} a^{i j} \in L_{\mathrm{loc}}^{p}(\mu)$, $b^{i}, c \in L_{\mathrm{loc}}^{p}(\mu)$. Предположим, что

$$
\int_{B_{R}}\left[a^{i j} \partial_{x_{i}} \partial_{x_{j}} \varphi+\partial_{x_{i}} a^{i j} \partial_{x_{j}} \varphi+b^{i} \partial_{x_{i}} \varphi+c \varphi\right] d \mu=0 \quad \forall \varphi \in C_{0}^{\infty}\left(B_{R}\right) .
$$

Тогда $\mu$ имеет плотность из $W_{\mathrm{loc}}^{p, 1}\left(B_{R}\right)$, которая локально гёльдерова. 
А. Бенсусан [28; гл. II, теорема 5.5] доказал $W_{\text {loc }}^{p, 1}$-регулярность для вероятностных мер на $\mathbb{R}^{d}$, удовлетворяющих уравнению $L_{\mathrm{I}, b}^{*} \mu=0$ с $b=b_{1}+b_{2}$, где $b_{1}$ липшицево и $b_{2}$ ограничено, при дополнительном предположении о существовании некоторой функции Ляпунова.

Классическое неравенство Харнака дает следующее утверждение.

СлеДСтвиЕ 1.2.11. В ситуации следствия 1.2 .8 пусть $b^{i} \in L_{\mathrm{loc}}^{p}(\Omega, d x)$, мера $\mu$ неотрицательна и $\varrho$ - ее непрерывная плотность. Тогда для кажсдого компактного множества $K$, содержащегося в связном открытом множестве $U$ с компактным замыканием в $\Omega$, мы имеем

$$
\sup _{K} \varrho \leqslant C \inf _{K} \varrho
$$

где постоянная $C$ зависит только от $\left\|a^{i j}\right\|_{W^{p, 1}(U)},\|b\|_{L^{p}(U)}, \inf _{U} \operatorname{det} A u K$ (если $K$ - иар, то зависимость от $K$ осуществляется через расстояние от $K$ до $\partial U)$. В частности, функция $\varrho$ не обращается в нуль в $U$, если она не равна нулю тождественно в $U$.

Зависимость $C$ от указанных величин будет изучена ниже в п. 1.9.

СлеДСтвиЕ 1.2.12. Предположим, что в ситуации следствия 1.2 .8 мъ имеем $b^{i} \in L_{\mathrm{loc}}^{p}(\Omega, d x)$ u $c \leqslant 0$. Предположим также, что непрерьвная плот-

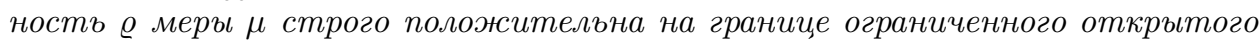
множества $U \subset \bar{U} \subset \Omega$. Тогда @ строго положительна на $U$.

Ясно, что условие $b^{i} \in L_{\mathrm{loc}}^{p}(\Omega, d x)$ в следствии 1.2.11 и следствии 1.2.12 нельзя заменить на альтернативное предположение $b^{i} \in L_{\mathrm{loc}}^{p}(\Omega, \mu)$ из следствия 1.2.8. Действительно, достаточно взять $b=\nabla \varrho / \varrho$ так, что $\varrho-$ вероятностная плотность, имеющая нули, но $|b| \in L^{p}(\mu)$ (например, можно взять плотность $\varrho$, которая ведет себя как $\exp \left(-x^{-2}\right)$ в окрестности нуля).

Следствие 1.2.8 можно обобщить следующим образом.

СЛЕДСТвИЕ 1.2.13. Пусть $p>d, a^{i j} \in W_{\mathrm{loc}}^{p, 1}(\Omega), b^{i}, f^{i}, c \in L_{\mathrm{loc}}^{p}(\Omega), i, j=$ $1, \ldots, d$, и пусть $A^{-1}$ локально равномерно ограничено в $\Omega$. Предположим, что $\mu$ - локально конечная борелевская мера на $\Omega, b^{i}, c \in L_{\mathrm{loc}}^{1}(\Omega, \mu)$ и для каждой функиии $\varphi \in C_{0}^{\infty}(\Omega)$ мы имеем

$$
\int_{\Omega}\left[a^{i j} \partial_{i} \partial_{j} \varphi+b^{i} \partial_{i} \varphi+c \varphi\right] d \mu=\int_{\Omega} f^{i} \partial_{i} \varphi d x .
$$

Тогда $\mu$ имеет плотность из $W_{\mathrm{loc}}^{p, 1}(\Omega)$.

ЗАмЕчАНИЕ 1.2.14. Легко видеть, что в следствии 1.2 .8 нельзя опустить предположения, что $A^{-1}$ локально равномерно ограничено и $a^{i j} \in W_{\mathrm{loc}}^{p, 1}$. Действительно, если $A$ и $b$ обращаются в нуль в точке $x_{0}$, то дираковская мера в $x_{0}$ удовлетворяет нашему эллиптическому уравнению. В частности, если заранее не дано, что мера $\mu$ абсолютно непрерывна, то нельзя брать произвольные лебеговские версии $A$. Чтобы увидеть, что $\mu$ не может быть более регулярной, чем $A$ (что уже отмечалось во введении), возьмем вероятностную меру $\mu$ с гладкой плотностью, которая удовлетворяет уравнению $L_{\mathrm{I}, b_{0}}^{*} \mu=0$, например, пусть $\mu$ - стандартная гауссовская мера и $b_{0}(x)=-x$. Если теперь $g-$ 
любая борелевская функция с $1 \leqslant g \leqslant 2$, то мера $g \cdot \mu$ удовлетворяет уравнению $L_{A, b}^{*} \mu=0$ с $A=g^{-1} \mathrm{I}$ и $b=g^{-1} b_{0}$. В частности, получаем пример, в котором $A$ и $b$ гёльдеровы и $A^{-1}$ равномерно ограничено, но плотность $\mu$ не является слабо дифференцируемой. Кроме того, условие $p>d$ существенно для включения $\mu$ в класс Соболева, даже если $A=\mathrm{I}$ (см. пример ниже). Однако если $\mu$ - вероятностная мера на $\mathbb{R}^{d}$, то условие $|b| \in L^{2}\left(\mathbb{R}^{d}, \mu\right)$ влечет, что $\mu=\varrho d x$, причем $\varrho \in W^{1,1}\left(\mathbb{R}^{d}\right)$ и $|\nabla \varrho|^{2} / \varrho \in L^{1}\left(\mathbb{R}^{d}\right)$ (см. п. 1.8).

ПримеР 1.2.15. Пусть $d>3$ и

$$
L^{*} F=\Delta F+\alpha \partial_{x^{i}}\left(x^{i}|x|^{-2} F\right)-F,
$$

где $\alpha=d-3$. Тогда функция $F(x)=\left(e^{r}-e^{-r}\right) r^{-(d-2)}, r=|x|$, локально интегрируема по Лебегу и $L^{*} F=0$ в смысле обобщенных функций, но $F$ не входит в $W_{\mathrm{loc}}^{2,1}\left(\mathbb{R}^{d}\right)$. Здесь

$$
b(x)=-\alpha x|x|^{-2}=\frac{\nabla\left(|x|^{-\alpha}\right)}{|x|^{-\alpha}}
$$

и $|b| \in L_{\text {lос }}^{d-\varepsilon}\left(\mathbb{R}^{d}\right)$ для всех $\varepsilon>0$. Аналогичным образом, если нет члена $-F$ в уравнении выше, то функция $F(x)=r^{-(d-3)}$ имеет те же самые свойства.

Для обоснования заметим, что $\partial_{x^{i}} F, \partial_{x^{i}} \partial_{x^{j}} F$ локально интегрируемы по Лебегу. Значит, уравнение $L^{*} F=0$ легко вытекает из уравнения

$$
f^{\prime \prime}+\frac{d-1+\alpha}{r} f^{\prime}+\alpha \frac{d-2}{r^{2}} f-f=0
$$

на $(0, \infty)$, которое выполнено для функции $f(r)=\left(e^{r}-e^{-r}\right) r^{-(d-2)}$. Остается заметить, что $F, \nabla F$ и $\Delta F$ локально интегрируемы по Лебегу, поскольку $f(r) r^{d-1}, f^{\prime}(r) r^{d-1}, f^{\prime \prime}(r) r^{d-1}$ локально ограничены, но $\nabla F$ не является квадратично интегрируемым по Лебегу в нуле. Если $d \geqslant 6$, то также и функция $F$ не является квадратично интегрируемой по Лебегу в нуле. В случае без члена $-F$ в уравнении похожие вычисления показывают, что $F(x)=r^{-(d-3)}$ имеет те же самые свойства.

Следующая теорема полезна при изучении локальных свойств плотностей, в частности, для контроля различных постоянных, появляющихся в локальных оценках. Она уточняет один частный случай более общего результата, который был сформулирован Ч. Морри в его книге [29; с. 156] не вполне корректным образом (с $\left.\Omega^{\prime}=\Omega\right)$. Приводимое ниже утверждение с $\Omega^{\prime}=\Omega$ было бы неверно, например, для уравнения Лапласа в шаре. Доказательство оценки Морри с исследованием зависимости постоянной от коэффициентов было проведено в [30] с той же самой неточностью, что и в [29]. Фактически рассуждения из [30] дают именно приводимую ниже оценку, как объяснено в [31], а оценка с $\Omega=\Omega^{\prime}$ возможна лишь для решений с нулевым граничным условием на области с достаточно регулярной границей. Отметим, что в существующих применениях теоремы Морри фактически использовалось лишь приводимое ниже верное утверждение, хотя в некоторых работах оно формулировалось с указанной неточностью (см., например, [8] и [9]). Доказательство дано в [22] и [31], где установлен более общий факт. 
Теорема 1.2.16. Предположим, что $\Omega \subset \mathbb{R}^{d}$ - ограниченная область, $A \in$ $C^{0, \delta}(\Omega)$, где $\delta>0$, причем существует такое число $\alpha>0$, что $A(x) \geqslant \alpha \cdot \mathrm{I}$ для всех $x \in \Omega$. Пусть $h^{i} \in L^{q}(\Omega)$, где $q>d$. Если функиия $u \in W^{q, 1}(\Omega)$ удовлетворяет уравнению $\partial_{x_{i}}\left(a^{i j} \partial_{x_{j}} u+h^{i}\right)=0$ в слабом смысле на $\Omega$, то для каждой области $\Omega^{\prime}$ с замыканием в $\Omega$ выполнена следующая оценка:

$$
\|u\|_{W^{q, 1}\left(\Omega^{\prime}\right)} \leqslant C\left(\|u\|_{L^{q}(\Omega)}+\|h\|_{L^{q}(\Omega)}\right), \quad h:=\left(h^{1}, \ldots, h^{d}\right),
$$

где число $C$ зависит только от $d, q, \alpha, \Omega, \Omega^{\prime} u\|A\|_{C^{0, \delta}}$.

Более того, если $u \in W^{q, 1}(\Omega)$ и $\partial_{x_{i}}\left(a^{i j} \partial_{x_{j}} u-b^{i} u+h^{i}\right)=0$ в слабом смысле и $b^{i} \in L^{q}(\Omega)$, то верна более сильная оценка

$$
\|u\|_{W^{q, 1}\left(\Omega^{\prime}\right)} \leqslant C\left(\|u\|_{L^{1}(\Omega)}+\|h\|_{L^{q}(\Omega)}\right),
$$

где $C$ зависит только от $d, q, \alpha, \Omega, \Omega^{\prime},\|A\|_{C^{0, \delta}} u\left\|b^{i}\right\|_{L^{q}(\Omega)}$.

ЗАмечАниЕ 1.2.17. Пусть борелевская мера $\mu$ на шаре $B_{R_{1}}$ радиуса $R_{1}>0$ в $\Omega$ удовлетворяет уравнению $L_{A, b}^{*} \mu+c \mu=f d x$, где $a^{i j}, b^{i}, c \in L^{1}\left(B_{R_{1}}, \mu\right)$, $f \in L^{p}\left(B_{R_{1}}\right)$ и

$$
A \geqslant \lambda_{1} \mathrm{I}, \quad\left\|a^{i j}\right\|_{W^{p, 1}\left(B_{R_{1}}\right)} \leqslant \lambda_{2}, \quad\left\|b^{i}\right\|_{L^{p}\left(B_{R_{1}}\right)}+\|c\|_{L^{p}\left(B_{R_{1}}\right)} \leqslant \lambda_{2}, \quad p>d .
$$

Тогда из результатов выше вытекает, что для всякого $R<R_{1}$ мера $\mu$ имеет непрерывную плотность $u \in W^{p, 1}\left(B_{R}\right)$, удовлетворяющую уравнению

$$
\partial_{i}\left(a^{i j} \partial_{j} u+\partial_{j} a^{i j} u-b^{i} u\right)+c u=f .
$$

Следовательно,

$$
\begin{aligned}
\|u\|_{W^{p, 1}\left(B_{R}\right)} & \leqslant \Lambda_{d, R_{1}}\left(\lambda_{1}, \lambda_{2}, R\right)\left[\|u\|_{L^{1}\left(B_{R_{1}}\right)}+\|f\|_{L^{p}\left(B_{R_{1}}\right)}\right] \\
& \leqslant \Lambda_{d, R_{1}}\left(\lambda_{1}, \lambda_{2}, R\right)\left[\operatorname{mes}\left(B_{R_{1}}\right) \sup _{B_{R_{1}}}|u|+\|f\|_{L^{p}\left(B_{R_{1}}\right)}\right],
\end{aligned}
$$

где $\Lambda_{d, R_{1}}\left(\lambda_{1}, \lambda_{2}, R\right)$ является локально ограниченной функцией на множестве $(0,+\infty)^{2} \times\left(0, R_{1}\right)$ и не зависит от $u$.

ПРЕДЛОЖЕНИЕ 1.2.18. Пусть $A_{k}=\left(a_{k}^{i j}\right)$ - последователъность непрерывных отображений на $\mathbb{R}^{d}$ со значениями в пространстве симметричных матрич и $b_{k}=\left(b_{k}^{i}\right)$ - последовательность борелевских векторных полей на $\mathbb{R}^{d}$. Предположим, что для каждого шара $B_{r} \subset \mathbb{R}^{d}$ найдутся такие числа $c_{r}>0$, $\alpha_{r}>0$ u $p=p_{r}>d$, , чmo

$$
A_{k} \geqslant c_{r} \mathrm{I}, \quad\left\|a_{k}^{i j}\right\|_{W^{p, 1}\left(B_{r}\right)}+\left\|b_{k}^{i}\right\|_{L^{p}\left(B_{r}\right)} \leqslant \alpha_{r} \quad \text { для всех } i, j, k .
$$

Предположим также, что существуют такие вероятностные мерь $\mu_{k}$ на $\mathbb{R}^{d}$, что $L_{A_{k}, b_{k}}^{*} \mu_{k}=0$. Тогда меры $\mu_{k}$ имеют непрерывные строго положительные плотности, которые равномерно гёльдеровы на каждом шаре. Если, кроме того, последовательность $\left\{\mu_{k}\right\}$ равномерно плотна, то она имеет компактное замыкание по вариационной норме, а каждая мера $\mu$ из ее замыкания имеет непрерывную строго положительную плотность, входящую в $W^{p, 1}\left(B_{r}\right)$ для каждого $r>0$. 
ДокАЗАТЕЛЬство. Из наших предположений и теоремы 1.2.6 вытекает, что меры $\mu_{k}$ имеют непрерывные плотности $f_{k}$. Поскольку функции $f_{k}-$ вероятностные плотности, мы получаем ввиду (1.2.2), что для каждого $r>0$ последовательность $\left\{f_{k}\right\}$ ограничена в $W^{p, 1}\left(B_{r}\right)$. По теореме вложения Соболева (см., например, [32]), последовательность $\left\{f_{k}\right\}$ равномерно гёльдерова на $B_{r}$, в частности, имеет компактное замыкание по sup-норме. Если $\left\{\mu_{k}\right\}$ равномерно плотна, то некоторая подпоследовательность $\left\{\mu_{k_{i}}\right\}$ сходится слабо к некоторой вероятностной мере $\mu$. Перейдя к подпоследовательности еще раз, можно считать, что функции $f_{k_{i}}$ сходятся равномерно на компактах и равномерно ограничены в $W^{p, 1}\left(B_{r}\right)$ для каждого $r>0$. Значит, $\mu$ имеет плотность $f \in W^{p, 1}\left(B_{r}\right)$. Теперь мы получаем непрерывную и строго положительную версию $f$. Следовательно, вероятностные меры $\mu_{k_{i}}$ сходятся к $\mu$ по вариации. Это рассуждение применимо ко всякой подпоследовательности в $\left\{\mu_{k}\right\}$, откуда мы получаем требуемое. Предложение доказано.

ЗАмЕчАНИЕ 1.2.19. Это предложение можно обобщить следующим образом. Пусть $\Omega$ - открытое множество в $\mathbb{R}^{d}$, являющееся объединением возрастающих открытых множеств $\Omega_{k}$ таких, что замыкание $\Omega_{k}$ есть компакт, содержащийся в $\Omega_{k+1}$. Пусть $\mu_{k}$ - вероятностные меры на $\Omega_{k}$, удовлетворяющие уравнениям $L_{A_{k}, b_{k}}^{*} \mu_{k}=0$ на $\Omega_{k}$, где $A_{k}$ - непрерывное отображение на $\Omega_{k}$ со значениями в множестве неотрицательных симметричных матриц, причем эти отображения локально равномерно невырождены и ограничены по $W^{p, 1}$-норме с $p>1$, и пусть $b_{k}$ - борелевские векторные поля на множествах $\Omega_{k}$, которые локально равномерно ограничены по норме из $L^{p}\left(\mathbb{R}^{d}\right)$. Тогда справедлив аналог заключения предыдущего предложения. Более того, то же самое верно для римановых многообразий размерности $d$.

В случае, когда матрицы диффузии $A$ бесконечно дифференцируемы, имеют место несколько более специальные результаты. В доказательствах мы воспользуемся следующей хорошо известной леммой.

Лемма 1.2.20. Предположим, что $a^{i j} \in C^{\infty}(\Omega) u \operatorname{det} A>0$.

(i) Пусть $r \in(-\infty, \infty)$ и $p>1$. Если и - такая обобщенная функиия, что $L_{A} u \in H_{\mathrm{loc}}^{p, r}(\Omega)$, то $u \in H_{\mathrm{loc}}^{p, r+2}(\Omega) ;$ также если $u \in H_{\mathrm{loc}}^{p, r}(\Omega)$, то $\partial_{x_{i}} u \in$ $H_{\mathrm{loc}}^{p, r-1}(\Omega), 1 \leqslant i \leqslant d$.

(ii) Верны включения $H_{\mathrm{loc}}^{p, 1}(\Omega) \subset L_{\mathrm{loc}}^{d p /(d-p)}(\Omega)$ u $L_{\mathrm{loc}}^{p}(\Omega) \subset H_{\mathrm{loc}}^{d p /(d-p),-1}(\Omega)$ при $1<p<d$ и $H_{\mathrm{loc}}^{p, 1}(\Omega) \subset C_{\mathrm{loc}}^{1-d / p}(\Omega)$ при $p>d$, так что в последнем случае элементы $H_{\mathrm{loc}}^{p, 1}(\Omega)$ локально ограничены. Кроме того, при $q>p>1$ имеем включение $L_{\mathrm{loc}}^{p}(\Omega) \subset H_{\mathrm{loc}}^{q, d / q-d / p}(\Omega)$.

(iii) Если $\mu$ - локально ограниченная радоновская мера на $\Omega$, то имеем $\mu \in$ $H_{\mathrm{loc}}^{p,-m}(\Omega)$ npu $p>1$ u $m>d(1-1 / p)$.

ДокАзАтЕльство. Утверждение (i) хорошо известно. Более точно, первое утверждение в (i) есть хорошо известный результат об эллиптической регулярности, а второе утверждение вытекает из ограниченности преобразований Рисса. Утверждение (ii) есть просто теорема вложения Соболева. Утверждение (iii) вытекает из этой теоремы вложения, ибо для регулярных подобластей $U$ в $\Omega$ мы имеем $H^{q, m}(U) \subset C(\bar{U})$ при $q m>d$, откуда по двойственности 
следует, что пространство $H^{q /(q-1),-m}(U)=\left[H^{q, m}(U)\right]^{*}$ содержит все ограниченные меры на $U$. Лемма доказана.

Мы формулируем следующий результат для $d>1$ только потому, что случай $d=1$ элементарен и уже обсуждался. Кроме того, мы включим в формулировку некоторые утверждения, которые следуют также из уже упомянутых результатов (доказательство - прямое и не использует результаты выше).

Теорема 1.2.21. При тех же предположениях относительно А, что и в лемме 1.2 .20$, пусть $d \geqslant 2 u \mu, \nu$ - радоновские меры (возможно, знакопеременные) на $\Omega$. Пусть отображение $b=\left(b^{i}\right): \Omega \rightarrow \mathbb{R}^{d}$ и функция $c: \Omega \rightarrow \mathbb{R}$ таковы, что $|b|, c \in L_{\mathrm{loc}}^{1}(\Omega, \mu)$. Предположим, что $L_{A, b, c}^{*} \mu=\nu$. Тогда справедливы следующие утверждения.

(i) Выполнено включение $\mu \in H_{\mathrm{loc}}^{p, 1-d(p-1) / p-\varepsilon}(\Omega)$ для всякого $p \geqslant 1$ и всякого $\varepsilon>0$. Здесь $1-d(p-1) / p>0$ при $p \in[1, d /(d-1))$, в частности, $\mu$ имеет плотность $F \in L_{\mathrm{loc}}^{p}(\Omega)$ для всякого $p \in[1, d /(d-1))$.

(ii) $E c \Omega u|b| \in L_{\mathrm{loc}}^{\gamma}(\Omega, \mu), c \in L_{\mathrm{loc}}^{\gamma / 2}(\Omega, \mu), \nu \in L_{\mathrm{loc}}^{d /(d-\gamma+2)}(\Omega)$, где $d \geqslant \gamma>1$, то $F:=d \mu / d x \in H_{\mathrm{loc}}^{p, 1}(\Omega)$ для всякого $p \in[1, d /(d-\gamma+1))$. В частности, $F \in L_{\mathrm{loc}}^{p}(\Omega)$ для всякого $p \in[1, d /(d-\gamma))$, где мы полагаем $d /(d-\gamma):=\infty$ при $\gamma=d$.

(iii) $Е с л и ~ \gamma>d$ и либо

(a) $|b| \in L_{\mathrm{loc}}^{\gamma}(\Omega), c, \nu \in L_{\mathrm{loc}}^{\gamma d(d+\gamma)}(\Omega)$, либо

(b) $|b| \in L_{\mathrm{loc}}^{\gamma}(\Omega, \mu), c \in L_{\mathrm{loc}}^{\gamma d /(d+\gamma)}(\Omega, \mu), \nu \in L_{\mathrm{loc}}^{\gamma d /(d+\gamma)}(\Omega)$,

то $\mu$ имеет плотность $F \in H_{\mathrm{loc}}^{\gamma, 1}(\Omega)$. В частности, $F \in C_{\mathrm{loc}}^{1-d / \gamma}(\Omega)$.

ДокАзАТЕЛЬСтво. (i) Мы имеем в смысле обобщенных функций

$$
\Delta \mu=\partial_{x^{i}}\left(b^{i} \mu\right)-c \mu+\nu
$$

на $\Omega$. Здесь по лемме 1.2 .20 (iii) правая часть входит в $H_{\mathrm{loc}}^{p,-m-1}(\Omega)$ при условии $m>d(1-1 / p)$. По лемме 1.2 .20 (i) заключаем, что $\mu \in H_{\text {loc }}^{p,-m+1}(\Omega)$, что приводит к нужному результату после подстановки $m=d(1-1 / p)+\varepsilon$.

Для доказательства утверждений (ii) и (iii) нам необходима некоторая подготовка. Зафиксируем $p_{1}>1$ и предположим, что $F=d \mu / d x \in L_{\text {loc }}^{p_{1}}(\Omega)$. Такое $p_{1}$ существует ввиду (i). Положим

$$
r:=r\left(p_{1}\right):=\frac{\gamma p_{1}}{\gamma-1+p_{1}}
$$

и заметим, что ввиду неравенств $1<\gamma$ и $p_{1}>1$ мы имеем $1<r<\gamma$. Далее, начав с формулы

$$
|b F|^{r}=\left(|b||F|^{1 / \gamma}\right)^{r}|F|^{r-r / \gamma}
$$

и используя неравенство Гёльдера (с $s=\gamma / r>1$ и $t:=s /(s-1)=\gamma /(\gamma-r))$ и включения $|b||F|^{1 / \gamma} \in L_{\mathrm{loc}}^{\gamma}(\Omega)$ и $F \in L_{\text {loc }}^{p_{1}}(\Omega)$, мы получим, что $b^{i} F \in L_{\mathrm{loc}}^{r}(\Omega)$. По лемме 1.2 .20 (i) имеем

$$
b^{i} F \in H_{\mathrm{loc}}^{r, 0}(\Omega), \quad\left(b^{i} F\right)_{x^{i}} \in H_{\mathrm{loc}}^{r,-1}(\Omega) .
$$

(ii) Положим

$$
q:=q\left(p_{1}\right):=\frac{\gamma p_{1}}{\gamma-2+2 p_{1}} \vee 1
$$


и заметим, что $q>1 \Leftrightarrow \gamma>2 \Leftrightarrow q<\gamma / 2$, в частности, $q<\gamma$ во всяком случае. Значит, повторяя описанное рассуждение с тройкой $c, \gamma / 2, q$ вместо $|b|, \gamma, r$, мы получаем, что

$$
c F \in L_{\mathrm{loc}}^{q}(\Omega) .
$$

Зафиксируем такое $p_{1}>1$, что $F:=d \mu / d x \in L_{\text {loc }}^{p_{1}}(\Omega)$. Пусть $r, q$ - такие же, как в (1.2.4) и (1.2.6) соответственно. Поскольку $\gamma \leqslant d$, то $q<d$, что ввиду $(1.2 .7)$ и утверждений (ii) и (iii) из леммы 1.2 .20 дает, что $c F \in H_{\mathrm{loc}}^{d q /(d-q),-1}(\Omega)$ при $q>1$ и что $c F \in H_{\mathrm{loc}}^{s,-1}(\Omega)$ для всякого $s \in(1, d /(d-1))$ при $q=1$.

Оказывается, что если $p_{1}<d /(d-\gamma)$, то

$$
c F \in H_{\mathrm{loc}}^{r,-1}(\Omega) .
$$

Действительно, если $q>1$, то (1.2.8) вытекает из того факта, что при $p_{1} \in$ $(1, d /(d-\gamma))$ выполнено неравенство $r \leqslant d q /(d-q)$. Если $q=1$, то $\gamma \leqslant 2$, и $(1.2 .8)$ вытекает из того факта, что $r<d /(d-\gamma+1) \leqslant d /(d-1)$ при $p_{1}<d /(d-\gamma)$.

Наконец, по лемме 1.2 .20 (ii) мы имеем $\nu \in H_{\mathrm{loc}}^{d /(d-\gamma+1),-1}(\Omega)$ при $\gamma>2$ и $\nu \in H_{\mathrm{loc}}^{s,-1}(\Omega)$ для всякого $s \in(1, d /(d-1))$ при $\gamma \leqslant 2$. Таким же образом, как и выше, получаем, что $\nu \in H_{\mathrm{loc}}^{r,-1}(\Omega)$ при $1<p_{1}<d /(d-\gamma)$. Вместе с $(1.2 .5)$ и (1.2.8) это показывает, что правая часть (1.2.3) теперь входит в $H_{\mathrm{loc}}^{r,-1}(\Omega)$. По лемме 1.2 .20 (i) мы имеем

$$
\mu \in H_{\mathrm{loc}}^{r, 1}(\Omega)
$$

и по лемме 1.2.20 (ii) мы имеем $F \in L_{\mathrm{loc}}^{p_{2}}(\Omega)$, где

$$
p_{2}:=\frac{d r}{d-r}=\frac{d \gamma p_{1}}{d \gamma-d+(d-\gamma) p_{1}}=: f\left(p_{1}\right)
$$

Итак, получаем

$$
p_{1} \in\left(1, \frac{d}{d-\gamma}\right) \quad \text { и } \quad F \in L_{\mathrm{loc}}^{p_{1}}(\Omega) \Longrightarrow F \in L_{\mathrm{loc}}^{f\left(p_{1}\right)}(\Omega)
$$

Легко проверить, что $p_{2}=f\left(p_{1}\right)>p_{1}$ при $p_{1}<d /(d-\gamma)$ и что единственное положительное решение уравнения $q=f(q)$ есть $q=d /(d-\gamma)$. Следовательно, взяв $p_{1}$ в интервале $(1, d /(d-1))$, что возможно в силу $(\mathrm{i})$, и положив $p_{k+1}=$ $f\left(p_{k}\right)$, получим возрастающую последовательность чисел $p_{k} \uparrow d /(d-\gamma)$, а это показывает, что $F \in L_{\mathrm{loc}}^{p}(\Omega)$ для всякого $p<d /(d-\gamma)$.

Однако при $p_{k} \nearrow d /(d-\gamma)$ последовательность чисел $r\left(p_{k}\right)$, определенная согласно (1.2.4), возрастает к

$$
\frac{\gamma \frac{d}{d-\gamma}}{\gamma-1+\frac{d}{d-\gamma}}=\frac{d}{d-\gamma+1}
$$

Ввиду (1.2.9) это доказывает (ii). 
(iii) Сначала рассмотрим случай (b). В силу последнего утверждения в (ii) мы имеем $F \in L_{\text {loc }}^{p_{1}}(\Omega)$ для всякого конечного $p_{1}>1$. Пусть $r:=r\left(p_{1}\right)$ определено как в (1.2.4). Тогда $1<r<\gamma$ и выполнено (1.2.5). Положим

$$
q:=q\left(p_{1}\right):=\frac{\frac{d \gamma}{d+\gamma} p_{1}}{\frac{d \gamma}{d+\gamma}-1+p_{1}} .
$$

Если $2 \leqslant d<\gamma$, то $d \gamma /(d+\gamma)>1$. Следовательно, поскольку $p_{1}>1$, мы получаем, что $1<q<d \gamma /(d+\gamma)$. Значит, повторяя рассуждения, приведшие к (1.2.5), с тройкой $c, d \gamma /(d+\gamma), q$ вместо $|b|, \gamma, r$, мы получим $c F \in L_{\mathrm{loc}}^{q}(\Omega)$, откуда $c F \in H_{\mathrm{loc}}^{d q /(d-q),-1}(\Omega)$ ввиду утверждения (ii) леммы 1.2.20. Заметим, что при $p_{1} \rightarrow \infty$ мы имеем

$$
r \uparrow \gamma, \quad q \uparrow \frac{d \gamma}{d+\gamma}, \quad \frac{d q}{d-q} \uparrow \gamma .
$$

Следовательно, комбинируя это с нашим предположением, что $\nu$ входит в класс $L_{\mathrm{loc}}^{d \gamma /(d+\gamma)}(\Omega)$, содержащийся в $H_{\mathrm{loc}}^{\gamma,-1}(\Omega)$ по утверждению (ii) леммы, и беря $p_{1}$ достаточно большим, мы видим, что правая часть (1.2.3) входит в $H_{\mathrm{loc}}^{\gamma-\varepsilon,-1}(\Omega)$ для всякого $\varepsilon \in(0, \gamma-1)$. По лемме 1.2 .20 (ii) заключаем, что $F \in H_{\mathrm{loc}}^{\gamma-\varepsilon, 1}(\Omega)$. Поскольку $\gamma>d$, функция $F$ локально ограничена. Теперь мы видим, что выше можно взять $p_{1}=\infty$, следовательно, правая часть $(1.2 .3)$ входит в $H_{\mathrm{loc}}^{\gamma,-1}(\Omega)$, что по утверждению (i) леммы дает нужный результат.

В остающемся случае (а) возьмем $p_{1}>\gamma /(\gamma-1)$ и предположим, что $F \in$ $L_{\text {loc }}^{p_{1}}(\Omega)$. Тогда вместо $(1.2 .4)$ и (1.2.10) положим

$$
r:=r\left(p_{1}\right):=\frac{\gamma p_{1}}{\gamma+p_{1}}, \quad q:=q\left(p_{1}\right):=\frac{\frac{d \gamma}{d+\gamma} p_{1}}{\frac{d \gamma}{d+\gamma}+p_{1}} \vee 1
$$

и заметим, что в силу оценки $p_{1}>\gamma /(\gamma-1)$ мы имеем $r>1$, что ввиду соотношения $p_{1}^{-1}+\gamma^{-1}=r^{-1}$ позволяет применить неравенство Гёльдера, начав с $|b F|^{r}=|b|^{r}|F|^{r}$, чтобы заключить, что выполнено (1.2.5). Поскольку $c \in$ $L_{\text {loc }}^{1}(\Omega, \mu)$,

$$
\frac{d \gamma}{d+\gamma}>1 \quad \text { и }\left(\frac{d \gamma}{d+\gamma}\right)^{-1}+p_{1}^{-1}=q^{-1},
$$

мы также имеем $c F \in L_{\text {loc }}^{q}(\Omega)$. Ясно, что $q<d$. Как и в части (ii), это дает $c F \in H_{\mathrm{loc}}^{d q /(d-q),-1}(\Omega)$ при $q>1$ и $c F \in H_{\mathrm{loc}}^{s,-1}(\Omega)$ для всякого $s \in(1, d /(d-1))$ при $q=1$. Мы утверждаем, что (1.2.8) выполнено (с $r=r\left(p_{1}\right)$, определенным в (1.2.11)) для всех $p_{1}>\gamma /(\gamma-1), p_{1} \neq d \gamma /(d \gamma-d-\gamma)$.

В самом деле, если $q>1$, то $d q /(d-q)=r$. Если $q=1$, то $p_{1} \leqslant d \gamma /(d \gamma-d-\gamma)$. Однако поскольку $p_{1} \neq d \gamma /(d \gamma-d-\gamma)$, то мы имеем $p_{1}<d \gamma /(d \gamma-d-\gamma)$, что равносильно неравенству $r<d /(d-1)$.

Итак, поскольку $\nu \in L_{\mathrm{loc}}^{d \gamma /(d+\gamma)}(\Omega) \subset H_{\mathrm{loc}}^{\gamma,-1}(\Omega) \subset H_{\mathrm{loc}}^{r,-1}(\Omega)$, ибо $r<\gamma$, то утверждение (i) леммы 1.2.20 дает следующее:

$$
\left(p_{1}>\frac{\gamma}{\gamma-1}, \quad p_{1} \neq \frac{d \gamma}{d \gamma-d-\gamma}, \quad F \in L_{\mathrm{loc}}^{p_{1}}(\Omega)\right) \quad \Longrightarrow \quad F \in H_{\mathrm{loc}}^{r, 1}(\Omega) .
$$


Если $r<d$, то последнее в свою очередь по утверждению (ii) леммы 1.2.20 влечет, что $F \in L_{\mathrm{loc}}^{p_{2}}(\Omega)$. Суммируем доказанное:

$$
\begin{gathered}
\left(p_{1}>\frac{\gamma}{\gamma-1}, \quad p_{1} \neq \frac{d \gamma}{d \gamma-d-\gamma}, \quad r:=\frac{\gamma p_{1}}{\gamma+p_{1}}<d, \quad F \in L_{\mathrm{loc}}^{p_{1}}(\Omega)\right) \\
\Longrightarrow F \in L_{\mathrm{loc}}^{p_{2}}(\Omega),
\end{gathered}
$$

где

$$
p_{2}:=\frac{d r}{d-r}=\frac{d \gamma p_{1}}{d \gamma-(\gamma-d) p_{1}}>\frac{d \gamma}{d \gamma-(\gamma-d)} p_{1} .
$$

Заметим также, что $\gamma /(\gamma-1)<d /(d-1)<d \gamma /(\gamma d-d-\gamma)$; таким образом, ввиду (i) найдется число $p_{1}$, с которого можно начать. Тогда, начав с $p_{1}$, достаточно близкого к $d /(d-1)$, итерациями (1.2.13) мы будем все время увеличивать $p$ посредством некоторого множителя, большего $d \gamma /(d \gamma-(\gamma-d))>1$. Осуществляя это, очевидным образом можно выбрать первое $p$ так, что итерированные числа $p$ никогда не будут равны $d \gamma /(d \gamma-d-\gamma)$ и соответствующие числа $r$ не будут совпадать с $d$. После нескольких шагов мы придем к ситуации, в которой $r>d$, и тогда мы заключим из (1.2.12), что $F$ является локально ограниченной (бесконечно итерировать (1.2.13) не удастся из-за ограничения $r<d)$. Как и в случае $(\mathrm{b})$, теперь легко завершить доказательство. Теорема 1.2.21 доказана.

Пример 1.2.15 показывает, что утверждение (iii) этой теоремы может быть неверным, если $\gamma>d$ заменить на $\gamma=d-\varepsilon$. Тогда функция $F$ даже не обязана входить в $H_{\mathrm{loc}}^{2,1}(\Omega)$.

ПРЕДЛОЖЕНИЕ 1.2.22. Пусть выполнены условия следствия 1.2 .8 и $\mu$ некоторая положительная мера на $\Omega$, являющаяся решением уравнения $L_{A, b, c}^{*} \mu=0$. Тогда всякое другое решение $\mu_{0}$ можно записать в виде $\mu_{0}=f \cdot \mu$, где $f \in W_{\mathrm{loc}}^{p, 2}(\Omega)$.

ДокАЗАтельство. Предположим сначала, что $d>1$. Тогда $p>2$. Мы знаем, что $\mu$ и $\mu_{0}$ имеют непрерывные плотности $\varrho$ и $\varrho_{0}$ соответственно из класса $W_{\text {loc }}^{p, 1}(\Omega)$ и что $\varrho$ не имеет нулей в $\Omega$. Положим $f=\varrho / \varrho_{0}$. Тогда $\mu_{0}=f \cdot \mu$ и $f \in W_{\mathrm{loc}}^{p, 1}(\Omega)$. Мы имеем $a^{i}:=\sum_{j=1}^{d} \partial_{x_{j}} a^{i j} \in L_{\mathrm{loc}}^{p}(\Omega)$. Пусть $a:=\left(a^{i}\right)$. Проверим, что $f$ удовлетворяет эллиптическому уравнению

$$
a^{i j} \varrho \partial_{x_{i}} \partial_{x_{j}} f+\langle\nabla f, 2 \varrho a+2 A \nabla \varrho-\varrho b\rangle=0
$$

в смысле слабых решений из класса $W_{\text {loc }}^{p, 1}(\Omega)$, т. е. в смысле тождества

$$
\int\left[-\varphi \partial_{x_{i}}\left(a^{i j} \varrho\right) \partial_{x_{j}} f-\langle\varrho A \nabla f, \nabla \varphi\rangle+\langle\nabla f, 2 \varrho a+2 A \nabla \varrho-\varrho b\rangle \varphi\right] d x=0
$$

для всех $\varphi \in C_{0}^{\infty}(\Omega)$. Это даст желаемое включение $f \in W_{\text {loc }}^{p, 2}(\Omega)$, поскольку мы имеем $\varrho a^{i j} \in W_{\mathrm{loc}}^{p, 1}(\Omega), \varrho A$ невырождено, $а \varrho, b \varrho, c \varrho \in L_{\mathrm{loc}}^{p}(\Omega)$. Чтобы установить (1.2.14), заметим, что равенство $L_{A, b, c}^{*} \mu_{0}=L_{A, b, c}^{*} \mu=0$ и формула 
интегрирования по частям дают тождества

$$
\begin{array}{r}
\int\left[-\partial_{x_{i}}\left(a^{i j} \varrho f\right) \partial_{x_{j}} \varphi+\langle f \varrho b, \nabla \varphi\rangle+c \varrho f \varphi\right] d x=0, \\
\int\left[-\partial_{x_{i}}\left(a^{i j} \varrho\right) \partial_{x_{j}} \varphi+\langle\varrho b, \nabla \varphi\rangle+c \varrho \varphi\right] d x=0
\end{array}
$$

для всех $\varphi \in C_{0}^{\infty}(\Omega)$. Поскольку $a^{i j}, \varrho, f \in W_{\mathrm{loc}}^{p, 1}(\Omega)$ и $p>2$, то получаем, что второе соотношение остается верным для всех функций $\varphi$ вида $\varphi=f \psi$ при $\psi \in C_{0}^{\infty}(\Omega)$. Это дает тождество

$$
\int\left[-\partial_{x_{i}}\left(a^{i j} \varrho\right) f \partial_{x_{j}} \varphi-\partial_{x_{i}}\left(a^{i j} \varrho\right) \varphi \partial_{x_{j}} f+\langle\varrho b, f \nabla \varphi\rangle+\langle\varrho b, \varphi \nabla f\rangle+c \varrho f \varphi\right] d x=0
$$

для всех $\varphi \in C_{0}^{\infty}(\Omega)$. Вычитая последнее равенство из (1.2.15) и дифференцируя произведения по формуле Лейбница, приходим к (1.2.14). В случае $d=1$ это рассуждение неприменимо при $p<2$, но в этом случае работает простое прямое доказательство: мы имеем $(A f \varrho)^{\prime}=f \varrho b+\psi$ и $(A \varrho)^{\prime}=b \varrho+k$, где $\psi$ есть неопределенный интеграл от $c f \varrho$ и $k-$ постоянная. Тогда $f^{\prime}=(\psi-k f)(A \varrho)^{-1}$. Предложение доказано.

Мы уже видели, что ввиду неравенства Харнака непрерывная версия плотности неотрицательной меры $\mu$, удовлетворяющей уравнению $L_{A, b}^{*} \mu=0$ в шаре $\Omega$, строго положительна при условии, что $a^{i j} \in W_{\mathrm{loc}}^{p, 1}(\Omega), A$ строго положительно и $b^{i} \in L_{\mathrm{loc}}^{p}(\Omega)$ при некотором $p>d$. В отличие от результата о соболевской регулярности, последнее условие на $b$ не может быть заменено альтернативным предположением, что $b^{i} \in L_{\mathrm{loc}}^{p}(\mu)$. Однако следующий результат С. В. Шапошникова [33] дает достаточное условие строгой положительности плотностей в терминах лишь интегрируемости относительно $\mu$.

Теорема 1.2.23. Пусть $\mu$ - ненулевая неотрицательная борелевская мера на шаре $\Omega$, удовлетворяющая уравнению $L_{\mathrm{I}, b}^{*} \mu=0$, где $\exp (\varepsilon|b|) \in L_{\mathrm{loc}}^{1}(\mu)$ для некоторого $\varepsilon>0$. Тогда $\mu$ имеет непреръвную строго положительную плотность.

В п. 1.9 мы обсудим нижние оценки плотностей при похожих предположениях глобальной экспоненциальной интегрируемости.

Упомянем ряд работ, где получены различные результаты, связанные со слабыми эллиптическими уравнениями для мер (или уравнениями для функций, которым удовлетворяют плотности решений уравнений для мер): [34]-[40]. Наконец, отметим, что результаты о локальной регулярности могут быть использованы для усиления полученных в [41] результатов о гладкости инвариантных мер относительно параметра.

1.3. Некоторые априорные оценки. В этом пункте мы установим некоторые общие априорные оценки, которые будут полезны при доказательстве существования решений. Эти оценки обеспечивают ограничения на интегралы относительно неизвестных решений для некоторых заданных функций.

Теорема 1.3.1. Пусть $V \geqslant 0$ - непреръвная функиия класса $W_{\mathrm{loc}}^{2,1}\left(\mathbb{R}^{d}\right)$, множество $U:=\{V<c\}$ ограничено, и пусть $\mu$ - неотрицательная борелевская мера на $U$, удовлетворяющая уравнению $L_{A, b}^{*} \mu=0$, где $A=\left(a^{i j}\right)-$ 
отображение на $U$ со значениями в пространстве положительных симметричных линейных операторов на $\mathbb{R}^{d}, a^{i j} \in W_{\operatorname{loc}}^{p, 1}(U)$ для некоторого $p>d$, $b=\left(b^{i}\right)$ - борелевское отображение из $U$ в $\mathbb{R}^{d}$, причем либо $|b| \in L_{\mathrm{loc}}^{p}(\mu)$, либо $|b| \in L_{\mathrm{loc}}^{p}(U)$. Предположим, что существуют такие борелевская функиия $\Psi \in L^{1}(\mu)$ и борелевская функиия $\Phi \geqslant 0$, что

$$
L V \leqslant \Psi-\Phi \quad \mu-\text { н.в. на } U
$$

Тогда

$$
\int_{U} \Phi d \mu \leqslant \int_{U} \Psi d \mu
$$

Если $\mu$ - вероятностная мера на $\mathbb{R}^{d}$, удовлетворяющая уравнению $L_{A, b}^{*} \mu=0$ на всем пространстве, где $A$ и $b$ удовлетворяют указанным выше предположениям локально, причем $L V \leqslant \Psi-\Phi$ на $\mathbb{R}^{d}$, где все множества $\{V \leqslant c\}$ компактны, то

$$
\int_{\mathbb{R}^{d}} \Phi d \mu \leqslant \int_{\mathbb{R}^{d}} \Psi d \mu .
$$

ДокАзАтЕльство. Достаточно доказать наше утверждение для ограниченных $\Phi$. В самом деле, как только это сделано, мы рассмотрим функции $\Phi_{k}=$ $\min (\Phi, k)$, для которых $L V \leqslant \Psi-\Phi_{k}$, и применим теорему Фату. Считая, что функция $\Phi$ ограничена, для данного $\varepsilon>0$ найдем такое компактное множество $K \subset U$, что $\mu(U)<\mu(K)+\varepsilon$ и

$$
\int_{U} \Phi d \mu<\int_{K} \Phi d \mu+\varepsilon .
$$

В силу непрерывности $V$ существует такое $r<c$, что

$$
K \subset\{V<r\} \subset\{V \leqslant r\} \subset U .
$$

Мы знаем, что $\mu$ имеет непрерывную плотность $\varrho$. Значит, найдется такое число $r_{1}<r$, что $K \subset\left\{V<r_{1}\right\}$ и

$$
\int_{\left\{r_{1} \leqslant V \leqslant r\right\}}|\langle\nabla V, b\rangle| d \mu<\varepsilon .
$$

В случае $d>1$ это вытекает из неравенства Коши-Буняковского, а если $d=1$, то $V^{\prime}$ функция локально ограничена. Возьмем такую функцию $\varphi$ на вещественной прямой, что $\varphi(t)=t$ при $t \leqslant r_{1}, \varphi(t)=\left(r_{1}+r\right) / 2$ при $t \geqslant r$, $\varphi^{\prime \prime}(t)=-1 /\left|r-r_{1}\right|$ на $\left[r_{1}, r\right]$ и $\varphi^{\prime \prime}(t)=0$ вне $\left[r_{1}, r\right]$. Тогда $0 \leqslant \varphi^{\prime} \leqslant 1, \varphi^{\prime \prime} \leqslant 0$,

$$
L_{A, b}(\varphi \circ V)=\varphi^{\prime \prime}(V)\langle A \nabla V, \nabla V\rangle+\varphi^{\prime}(V) L_{A, b} V,
$$

что равно нулю вне $\{V \leqslant r\}$, поскольку функция $\varphi \circ V$ постоянна вне $\{V \leqslant r\}$, и $L_{A, b}(\varphi \circ V)=L_{A, b} V$ на $\left\{V \leqslant r_{1}\right\}$. Так как $\varphi \circ V \in W_{\text {loc }}^{2,1}(U)$ и функция $\varphi \circ V$ постоянна вне $\{V \leqslant r\}$, то

$$
\int_{U} L_{A, b}(\varphi \circ V) d \mu=0 .
$$


Учитывая, что $\varphi^{\prime \prime}(V) \leqslant 0$ и $0 \leqslant \varphi^{\prime}(V) \leqslant 1$, получаем

$$
\begin{aligned}
\int_{\left\{V \leqslant r_{1}\right\}}[\Psi-\Phi] d \mu & \geqslant \int_{\left\{V \leqslant r_{1}\right\}} L(\varphi \circ V) d \mu=-\int_{\left\{r_{1}<V \leqslant r\right\}} L(\varphi \circ V) d \mu \\
& \geqslant-\int_{\left\{r_{1}<V \leqslant r\right\}}|\langle\nabla V, b\rangle| d \mu \geqslant-\varepsilon,
\end{aligned}
$$

откуда

$$
\int_{\left\{V \leqslant r_{1}\right\}} \Phi d \mu \leqslant \int_{\left\{V \leqslant r_{1}\right\}} \Psi d \mu+\varepsilon .
$$

Поскольку $\varepsilon>0$ было произвольно и числа $r$ и $r_{1}$ можно выбрать сколь угодно близкими к $c$, то желаемая оценка доказана. Утверждение относительно всего пространства сразу вытекает из этого, ибо можно взять возрастающие множества $\{V<j\}$ с объединением $\mathbb{R}^{d}$. Теорема доказана.

Напомним, что функция $V$ называется компактной, если все множества $\{V \leqslant c\}$ компактны. Для непрерывной функции $V$ на $\mathbb{R}^{d}$ это равносильно тому, что $\lim _{|x| \rightarrow \infty} V(x)=+\infty$. Будем называть функцию $V$ квазикомпактной, если пространство можно представить в виде объединения возрастающих компактов $\left\{V \leqslant c_{k}\right\}$ для некоторой возрастающей последовательности чисел $c_{k}$. Например, квазикомпактна четная непрерывная функция на прямой, возрастающая на $[0,+\infty)$ к некоторому $c$, но не принимающая значение $c$.

Теорема 1.3.2. Предположим, что даны такие отображения $A_{k}=\left(a_{k}^{i j}\right)$ на $\mathbb{R}^{d}$ со значениями в пространстве положительных симметричных линейных операторов на $\mathbb{R}^{d}$ и борелевские векторные поля $b_{k}=\left(b_{k}^{i}\right)$ на $\mathbb{R}^{d}$, что для каждого $R>0$ найдутся числа $\alpha_{R}>d, \beta_{R}>0 u \gamma_{R}>0$, для которьх

$$
\begin{gathered}
\kappa_{R}:=\sup _{k} \int_{|x| \leqslant R}\left[\left|b_{k}(x)\right|^{\alpha_{R}}+\sum_{i, j=1}^{d}\left\|\nabla a_{k}^{i j}(x)\right\|^{\alpha_{R}}\right] d x<\infty, \\
\beta_{R} \mathrm{I} \leqslant A_{k}(x) \leqslant \gamma_{R} \mathrm{I} \quad \forall k, \forall x \in\{y:|y| \leqslant R\} .
\end{gathered}
$$

Предположим также, что существует такая непрерывная квазикомпактная (например, компактная) функиия $V \in W_{\mathrm{loc}}^{2,1}\left(\mathbb{R}^{d}\right)$, что

$$
\lim _{|x| \rightarrow \infty} \sup _{k} L_{A_{k}, b_{k}} V(x)=-\infty .
$$

Пусть $\left\{\mu_{k}\right\}$ - последовательность таких вероятностных мер на $\mathbb{R}^{d}$, что

$$
L_{A_{k}, b_{k}}^{*} \mu_{k}=0 \text {. }
$$

Положим $U_{R}:=\{V<R\}$. Тогда справедливы следующие утверждения.

(i) Меры $\mu_{k}$ имеют такие непрерывные плотности $\varrho_{k}$, что для каждого фиксированного $R>1$ функции $\left.\varrho_{k}\right|_{U_{R}}$ равномерно ограничены, равномерно гёлъдеровы и равномерно ограничены в $W^{\alpha_{R}, 1}\left(U_{R-1}\right)$.

(ii) Для каждого $R>0$ имеем $\inf _{k} \inf _{x \in U_{R}} \varrho_{k}(x)>0$.

(iii) Последовательность $\left\{\mu_{k}\right\}$ относительно слабо компактна на $\mathbb{R}^{d}$. 
Более того, предположим, что для каждого $k$ вероятностная мера $\mu_{k}$ определена на $U_{k}=\left\{V<c_{k}\right\}$, где $c_{k} \uparrow \infty$, и удовлетворяет нашему уравнению $L_{A_{k}, b_{k}}^{*} \mu_{k}=0$ лишь в $U_{k}$. Тогда утверждения (i)-(iii) остаются справедливыми со следуюшими изменениями: в (i) и (ii) мы рассматриваем $k$, для которых $c_{k}>R$.

ДокАзАтельство. Утверждения (i) и (ii) вытекают непосредственно из результатов в п. 1.2. Чтобы доказать (iii), возьмем такое компактное множество $E$, что

$$
L_{A_{k}, b_{k}} V(x) \leqslant-1 \quad \forall k, \forall x \notin E .
$$

Пусть $\Psi_{k}:=\left|L_{A_{k}, b_{k}} V\right| I_{E}$ и $\Phi_{k}:=-\left(L_{A_{k}, b_{k}} V\right) I_{\mathbb{R}^{d} \backslash E}$. Тогда $L_{A_{k}, b_{k}} V \leqslant \Psi_{k}-\Phi_{k}$ и $\Phi_{k} \geqslant 0$, поскольку $\Phi_{k}=-L_{A_{k}, b_{k}} V$ вне $E$. Теорема 1.3.1 дает

$$
\int_{\mathbb{R}^{d}} \Phi_{k} d \mu_{k} \leqslant \int_{\mathbb{R}^{d}} \Psi_{k} d \mu_{k} .
$$

Из наших предположений вытекает, что

$$
S:=\sup _{k} \int_{E}\left|L_{A_{k}, b_{k}} V\right| d \mu_{k}<\infty .
$$

Следовательно, предыдущая оценка дает

$$
\int_{\mathbb{R}^{d} \backslash E}\left|L_{A_{k}, b_{k}} V\right| d \mu_{k} \leqslant S,
$$

откуда находим

$$
\sup _{k} \int_{\mathbb{R}^{d}}\left|L_{A_{k}, b_{k}} V\right| d \mu_{k} \leqslant 2 S
$$

Следовательно, для заданного $C>0$ можно найти такое $R$, что $L_{A_{k}, b_{k}} V \leqslant-C$ вне $\{V \leqslant R\}$ для всех $k$. Значит, для всех $k$ имеем

$$
\mu\left(\mathbb{R}^{d} \backslash\{V \leqslant R\}\right) \leqslant \frac{2 S}{C},
$$

что означает равномерную плотность $\left\{\mu_{k}\right\}$ и, следовательно, относительную слабую компактность $\left\{\mu_{k}\right\}$.

Докажем последнее утверждение, в котором каждая мера $\mu_{k}$ определена лишь на множестве $\left\{V<c_{k}\right\}$. Никаких изменений в доказательстве утверждений (i) и (ii) не требуется, кроме того, что теперь надо рассматривать $k$, для которых $c_{k}>R$. То же самое доказательство (iii) работает также и в этом случае, надо лишь рассматривать достаточно большие $k$, для которых $E \subset\left\{V<c_{k}\right\}$. Теорема доказана.

СлеДствиЕ 1.3.3. В ситуачии теоремы 1.3 .2 последовательность $\left\{\mu_{k}\right\}$ относительно компактна по вариации.

ДокАЗАТЕЛЬство. Из теоремы 1.3 .2 вытекает, что каждая подпоследовательность этой последовательности имеет такую подпоследовательность $\left\{\nu_{k}\right\}$, что $\left\{\nu_{k}\right\}$ сходится слабо к некоторой вероятностной мере $\nu$, а непрерывные плотности $\varrho_{k}$ мер $\nu_{k}$ сходятся локально равномерно к непрерывному пределу $\varrho_{0}$. Ясно, что $\varrho_{0}$ служит плотностью для $\nu$. Следовательно, $\left\|\varrho_{k}-\varrho_{0}\right\|_{L^{1}\left(\mathbb{R}^{d}\right)} \rightarrow 0$, в чем и состоит наше утверждение. Следствие доказано. 
СлЕДСТВИЕ 1.3.4. Предположим, что условия теоремы 1.3.2 выполнены за исключением (1.3.3), которое заменяется условием

$$
\lim _{|x| \rightarrow \infty} \sup _{k}\left\langle b_{k}(x), x\right\rangle=-\infty .
$$

Предположим, кроме того, что $\sup _{R} \gamma_{R}<\infty$. Тогда для каждого $p \geqslant 1$ имеем

$$
M_{p}:=\sup _{k} \int_{\mathbb{R}^{d}}\langle x, x\rangle^{p} \mu_{k}(d x)<\infty .
$$

ДоказАтельство. Можно считать, что $p \in \mathbb{N}$. В силу (1.3.4) функция $V(x)=\langle x, x\rangle^{p}$ удовлетворяет условию (1.3.3), поскольку

$$
\begin{aligned}
& L_{A_{k}, b_{k}} V(x)= 4 p(p-1)\langle x, x\rangle^{p-2} \sum_{i, j=1}^{d} a_{k}^{i j}(x) x_{i} x_{j} \\
& \quad+2 p\langle x, x\rangle^{p-1} \operatorname{trace} A_{k}(x)+2 p\langle x, x\rangle^{p-1}\left\langle x, b_{k}(x)\right\rangle \\
& \leqslant\langle x, x\rangle^{p-1}\left[4 p(p-1)\left\|A_{k}(x)\right\|+2 p d\left\|A_{k}(x)\right\|+2 p\left\langle x, b_{k}(x)\right\rangle\right] .
\end{aligned}
$$

Ввиду (1.3.4) и равномерной ограниченности $\left\|A_{k}(x)\right\|$ найдутся $R>0$ и два положительных числа $c_{1}$ и $c_{2}$, для которых

$$
L_{A_{k}, b_{k}} V(x) \leqslant c_{1}-c_{2}\langle x, x\rangle^{p-1} \quad \forall k \geqslant 1
$$

при $|x|>R$. Из наших предположений и теоремы 1.3 .2 вытекает, что

$$
\sup _{k} \int_{\{|x| \leqslant R\}}\left|L_{A_{k}, b_{k}} V(x)\right| \mu_{k}(d x)<\infty .
$$

Значит, $M_{p-1}<\infty$ по теореме 1.3.1. Следствие доказано.

ЗАмЕЧАНИЕ 1.3.5. (i) Заметим, что доказательство плотности $\left\{\mu_{k}\right\}$ использовало лишь (1.3.3). Значит, утверждение (ii) теоремы 1.3 .2 справедливо при (1.3.3) для мер $\mu_{k}$, удовлетворяющих уравнениям $L_{A_{k}, b_{k}}^{*} \mu_{k}=0$ с произвольными коэффициентами $A_{k}$ и $b_{k}$, которые локально $\mu_{k}$-интегрируемы.

(ii) Ясно, что условия (1.3.1) и (1.3.2) могут накладываться локально, т. е. для каждой точки $x$ существуют окрестность $W$ точки $x$ и числа $\alpha_{W}>d$, $\beta_{W}>0$ и $\gamma_{W}>0$ такие, что (1.3.1) и (1.3.2) выполнены с $W, \alpha_{W}, \beta_{W}$ и $\gamma_{W}$ вместо $U_{R}, \alpha_{R}, \beta_{R}$ и $\gamma_{R}$ соответственно.

1.4. Существование решений. Здесь мы представим достаточные условия существования решений, выраженные в терминах функций Ляпунова, а значит, проверяемые явно через коэффициенты наших операторов. Этот метод восходит к Р. 3. Хасьминскому [42], [43], а изложенные результаты заимствованы из [8] с некоторыми усилениями.

Теорема 1.4.1. Предположим, что $A=\left(a^{i j}\right)_{i, j \leqslant d}$-отображение на $\mathbb{R}^{d}$ со значениями в пространстве неотрицательных симметричных линейных операторов на $\mathbb{R}^{d}$ u $b: \mathbb{R}^{d} \rightarrow \mathbb{R}^{d}$ - борелевское отображение такие, что для каждого шара $U_{R}$ существуют числа $\alpha_{R}>d u \beta_{R}>0$, для которых $\beta_{R} \mathrm{I} \leqslant A(x)$ 
при всех $\left.x \in U_{R} u a^{i j}\right|_{U_{R}} \in W^{\alpha_{R}, 1}\left(U_{R}\right),\left.|b|\right|_{U_{R}} \in L^{\alpha_{R}}\left(U_{R}\right)$. Предположим, кроме того, что существует такая квазикомпактная (например, компактная) функиия $V \in C^{2}\left(\mathbb{R}^{d}\right)$, что

$$
L_{A, b} V(x) \rightarrow-\infty \quad \text { npu }|x| \rightarrow \infty .
$$

Тогда существует такая борелевская вероятностная мера $\mu$ на $\mathbb{R}^{d}$, что

$$
|b| \in L_{\mathrm{loc}}^{1}(\mu) \quad u \quad L_{A, b}^{*} \mu=0 .
$$

Более того, мера $\mu$ обладает такой непрерывной строго положительной

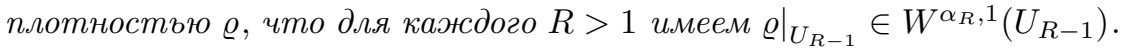

ДокАЗАтЕльство. По нашему условию на $V$ найдутся возрастающие области $U_{k}=\left\{V<c_{k}\right\}$ с компактными замыканиями $U_{k}$ в $U_{k+1}$, дающие в объединении все пространство. Сначала найдем последовательность неотрицательных функций $p_{k}$, которые не равны нулю тождественно и удовлетворяют нашему уравнению в областях $U_{k}$. Для этого возьмем шар $B_{k}$, содержащий $U_{k}$. Известно (см. [44; теорема 3.2 и замечание в конце §3]), что для каждого $k$ существует такая неотрицательная функция $p_{k} \in W^{\alpha_{k}, 1}\left(B_{k}\right)$, что $p_{k}=1$ на границе $B_{k}$ (в смысле, объясненном в [44]) и внутри $B_{k}$ имеем

$$
\partial_{x_{i}}\left(a^{i j} \partial_{x_{j}} p_{k}-b^{i} p_{k}+\left(\partial_{x_{j}} a^{i j}\right) p_{k}\right)=0
$$

в слабом смысле. Тот факт, что эти решения неотрицательны, вытекает из слабого принципа максимума [45; теорема 7]. Наш случай соответствует равенству $\gamma=b^{i}=b=0$ в указанной теореме (см. также [44; упражнение 8.1]). Стоит заметить, что существование функции $p_{k}$ с вышеупомянутыми свойствами может быть выведено из разрешимости граничной задачи для гладких коэффициентов. В самом деле, существует последовательность гладких отображений $A_{m}=\left(a_{m}^{i j}\right)_{i, j=1}^{d}$ на большем шаре $B_{k}^{\prime} \supset B_{k}$ со значениями в пространстве положительных симметричных линейных операторов на $\mathbb{R}^{d}$ такая, что отображения $A_{m}$ равномерно невырождены и сходятся к $A$ по норме класса Соболева $W^{\alpha_{k}, 1}\left(B_{k}^{\prime}\right)$. Кроме того, существует последовательность гладких отображений $b_{m}=\left(b_{m}^{i}\right)_{i=1}^{d}$, сходящаяся к $b$ в $L^{\alpha_{k}}\left(B_{k}^{\prime}, \mathbb{R}^{d}\right)$. Обозначим через $f_{m}$ решение граничной задачи

$$
\partial_{x_{i}}\left(a_{m}^{i j}(x) \partial_{x_{j}} f_{m}-b_{m}^{i} f_{m}+\partial_{x_{j}} a_{m}^{i j} f_{m}\right)=0
$$

с граничным условием $f_{m}=1$ на границе $B_{k}^{\prime}$. Такие решения существуют согласно [46; теорема 8.3 и замечание в конце $\S 8.2$ ]. Пусть $\kappa_{m}$ таково, что минимум $\tilde{f}_{m}:=\kappa_{m} f_{m}$ на $B_{k}^{\prime}$ равен 1 . Согласно результатам п. 1.2 , последовательность $\left\{\tilde{f}_{m}\right\}$ равномерно ограничена на $B_{k}$. Кроме того, ограничения функций $\tilde{f}_{m}$ имеют равномерно ограниченные нормы в пространстве $W^{\alpha_{k}, 1}\left(B_{k}\right)$. Выбирая подпоследовательность из $\left\{\tilde{f}_{m}\right\}$, которая равномерно сходится на $B_{k}$, заключаем, что ее предел после нормировки может быть взят в качестве $p_{k}$, поскольку он удовлетворяет уравнению (1.4.2) в $B_{k}$ в слабом смысле и оценивается снизу числом 1. Из нашего рассуждения вытекает, что $p_{k} \in W^{\alpha_{k}, 1}\left(U_{k}\right)$. Умножая решения $p_{n_{k}}$ на положительные постоянные и полагая $p_{k}=0$ вне $U_{k}$, 
мы получим вероятностные меры $\mu_{k}$, удовлетворяющие эллиптическим уравнениям $L_{A, b}^{*} \mu_{k}=0$ в $U_{k}$. Согласно теореме 1.3 .2 , последовательность $\left\{\mu_{k}\right\}$ содержит подпоследовательность $\left\{\mu_{k_{i}}\right\}$, сходящуюся по вариации к вероятностной мере $\mu$ со строго положительной непрерывной плотностью $\varrho$. Кроме того, для каждого шара $B$ найдется такое число $k_{0}$, что плотности $\varrho_{k_{i}}$ мер $\mu_{k_{i}}$ с $k_{i} \geqslant k_{0}$ сходятся равномерно на $B$. Следовательно, для всякого $\varphi \in C_{0}^{\infty}\left(\mathbb{R}^{d}\right)$ получаем по теореме Лебега об ограниченной сходимости

$$
0=\lim _{i \rightarrow \infty} \int_{\mathbb{R}^{d}} L_{A, b} \varphi(x) \varrho_{k_{i}}(x) d x=\int_{\mathbb{R}^{d}} L_{A, b} \varphi(x) \varrho(x) d x .
$$

Таким образом, $\mu=\varrho d x$ есть искомое решение. Теорема доказана.

СлеДСтвиЕ 1.4.2. Заключение предыдущей теоремъ верно, если A - локально липшицево равномерно ограниченное отображение со значениями 8 пространстве положительных симметричных операторов на $\mathbb{R}^{d} u b$ - борелевское отображение, для которого $|b| \in L_{\mathrm{loc}}^{\alpha}\left(\mathbb{R}^{d}\right)$, где $\alpha>d$, причем

$$
\lim _{|x| \rightarrow \infty}\langle b(x), x\rangle=-\infty .
$$

ДокАЗАТЕЛЬСтво. Достаточно взять $V(x)=\langle x, x\rangle$.

Например, предыдущее следствие применимо к случаю, когда $A=\mathrm{I}$ и $b(x)=$ $-k(x) x$, где $k \in L_{\mathrm{loc}}^{\alpha}\left(\mathbb{R}^{d}\right)$ таково, что

$$
k(x)|x|^{2} \rightarrow+\infty \quad \text { при }|x| \rightarrow \infty .
$$

Более общим образом, если $A=\mathrm{I}$, то достаточно иметь более слабую оценку

$$
\limsup _{|x| \rightarrow \infty}\langle x, x\rangle^{\gamma-1}[2(\gamma-1)+d+\langle b(x), x\rangle]=-\infty
$$

для некоторого $\gamma \geqslant 1$ (здесь функция $V(x)=\langle x, x\rangle^{\gamma}$ может быть использована).

Другой пример, покрываемый теоремой 1.4.1, таков: $A=\mathrm{I}$ и для некоторого $\gamma \geqslant 1$ имеем $\langle b(x), x\rangle \leqslant-r<-d+2-2 \gamma$ вне некоторого шара (конечно, мы предполагаем, что $|b|$ локально интегрируемо в степени выше $d$ ). Тогда мы возьмем функцию $V(x)=\langle x, x\rangle^{\gamma}$. Такое же условие коэрцитивности предполагается в [47] для локально ограниченного $b$ (чтобы получить уравнение из [47], надо взять $A=\mathrm{I} / 2$ в нашем случае). Заметим, что, как показано в [47], к этому случаю можно свести более общий случай, где коэффициент диффузии непрерывен, невырожден и удовлетворяет некоторым равномерным оценкам, при условии, что снос локально ограничен и коэрцитивен и что соответствующее стохастическое дифференциальное уравнение имеет единственное слабое решение.

Следующий результат был доказан в [8].

СлеДСтвиЕ 1.4.3. Пусть $A=\left(a^{i j}\right)$ - непрерьвное отображение на $\mathbb{R}^{d}$ со значениями в пространстве положительных симметричных линейных операторов на $\mathbb{R}^{d}$ и $b$ - борелевское векторное поле на $\mathbb{R}^{d}$. Предположим, что существует такая квазикомпактная функиия $V \in C^{2}\left(\mathbb{R}^{d}\right)$, что выполнено (1.4.1). Тогда справедливы следующие утверждения. 
(i) Если b непрерывно, то существует вероятностная мера $\mu$, удовлетворяющая уравнению $L_{A, b}^{*} \mu=0$.

(ii) Если $\operatorname{det} A>0$ и мера $\mu$, которая имеет плотность из класса $L_{\mathrm{loc}}^{d /(d-1)}\left(\mathbb{R}^{d}\right)$ и удовлетворяет уравнению $L_{A, b}^{*} \mu=0$.

Замечание 1.4.4. Ясно, что в случае невырожденного $A$ борелевская измеримость $b$ может быть заменена лебеговской измеримостью и что наше решение не зависит от лебеговски эквивалентной версии $b$, так как всякое решение имеет плотность.

Заметим, что (1.4.1) выполнено, если имеются положительные числа $c_{1}$ и $c_{2}$, для которых

$$
L V(x) \leqslant c_{1}-c_{2} V(x) \quad \text { и } \quad \lim _{|x| \rightarrow \infty} V(x)=+\infty .
$$

В общем случае предположение, что $\lim _{|x| \rightarrow \infty} L_{A, b} V(x)=-\infty$, даже при $A=\mathrm{I}$ не может быть заменено более слабым предположением, что

$$
L_{A, b} V(x) \leqslant c<0 \quad \text { вне некоторого шара } U \text {. }
$$

Действительно, пусть $d=1, A=1$ и $b(x)=-\operatorname{sign} x /(1+|x|)$. Имеем $x b(x) \rightarrow-1$ при $|x| \rightarrow \infty$. Предположим, что $\mu$ - вероятностная мера, удовлетворяющая уравнению $L_{1, b}^{*} \mu=0$. Тогда $\mu$ имеет локально абсолютно непрерывную плотность $\varrho$, для которой $\varrho^{\prime \prime}+(b \varrho)^{\prime}=0$ в смысле обобщенных функций, откуда $\varrho^{\prime}+b \varrho=0$, поскольку функция $b \varrho$ интегрируема (достаточно проинтегрировать по интервалам $\left[t_{k}, s_{k}\right]$, где $\left.t_{k} \rightarrow-\infty, s_{k} \rightarrow+\infty, \varrho\left(t_{k}\right) \rightarrow 0, \varrho\left(s_{k}\right) \rightarrow 0\right)$. Кроме того, $\varrho>0$, так что $(\ln \varrho)^{\prime}=-(\ln (1+|x|))^{\prime}$, откуда получаем, что $\varrho(x)=c /(1+|x|)$, что является неинтегрируемой функцией. В [28] существование инвариантной вероятностной меры установлено в предположении (1.4.3) и дополнительном предположении, что $|\nabla V|^{2} / V \leqslant c$ для некоторого числа $c>0$. Однако в этом случае мы можем взять новую функцию Ляпунова $W=(2 V)^{\alpha}$, где $\alpha=1+c^{-1}$, которая удовлетворяет (1.4.1).

Ясно, что изложенные условия существования можно модифицировать для операторов дивергентного вида. Например, если функции $a^{i j}$ липшицевы и $\langle b(x), x\rangle \leqslant c_{1}-c_{2}|x| \ln |x|$ вне некоторого шара, то дополнительное слагаемое в коэффициенте сноса, которое появляется, когда мы записываем уравнение в недивергентном виде, не нарушает условие (1.4.1).

Другие достаточные условия существования, выраженные в терминах коэффициентов $A$ и $b$, см. в [48].

Заметим также, что в случае, когда $b$ является градиентом, имеются более специальные достаточные условия, гарантирующие существование вероятностных мер с логарифмическим градиентом $b$ (см. [26; § 7.5]), но даже в этом случае общие достаточные условия, представленные выше, оказываются весьма эффективными.

1.5. Ассоциированные $L^{1}$-полугруппы. Здесь мы обсудим полугруппы, ассоциированные с решениями наших эллиптических уравнений для мер, и связи между такими уравнениями и собственной инвариантностью мер относи- 
тельно ассоциированных полугрупп. Напомним, что во введении было определено понятие меры, инвариантной относительно полугруппы $\left(T_{t}\right)_{t \geqslant 0}$ ограниченных линейных операторов на пространстве $B_{b}(X)$ ограниченных измеримых функций (см. указанное там равенство (3)). Аналогично определяется инвариантность в случае, когда $X$ есть топологическое пространство, $\mu$ - борелевская мера на $X$ и $\left(T_{t}\right)_{t \geqslant 0}$ есть полугруппа ограниченных линейных операторов на пространстве $C_{b}(X)$ ограниченных непрерывных функций. Очевидная модификация последнего понятия возникает, если операторы $T_{t}$ суть просто операторы из $C_{b}(X)$ в $L^{\infty}(\mu)$, не обязательно образующие полугруппу. Если для всех ограниченных борелевских функций $f$ и $g$ имеем

$$
\int_{X} T_{t} f(x) g(x) \mu(d x)=\int_{X} f(x) T_{t} g(x) \mu(d x),
$$

то полугруппа называется симметричной, а мера $\mu$ называется симметричной инвариантной. Ясно, что этот случай характеризуется тем свойством, что генератор $L$ полугруппы симметричен в $L^{2}(\mu)$. Если $\left(T_{t}\right)_{t \geqslant 0}-$ переходная полугруппа марковского процесса, то этот процесс называется $\mu$-симметричным. Процесс называется симметризуемым, если существует такая мера $\mu$, что он $\mu$-симметричен.

Нас интересует случай, когда полугруппа $\left(T_{t}\right)_{t \geqslant 0}$ в некотором смысле порождена эллиптическим оператором $L_{A, b}$, для которого существует вероятностная мера $\mu$, удовлетворяющая уравнению $L_{A, b}^{*} \mu=0$. Однако следует быть весьма осторожными с возможной интерпретацией термина "порождена". Мы будем вместо этого использовать термин "ассоциированная полугруппа", что будет означать просто следующее: полугруппа $\left(T_{t}\right)_{t \geqslant 0}$ на пространстве ограниченных борелевских функций будет называться ассоциированной с $L_{A, b}$ и $\mu$, если она продолжается до сильно непрерывной полугруппы на $L^{1}(\mu)$, генератор которой совпадает с $L_{A, b}$ на классе гладких функций с компактным носителем. В типичных случаях такая полугруппа не является непрерывной на $B_{b}$ или на $C_{b}$. Более того, в общем случае она не единственна (мы увидим такие примеры, однако будет выделена некоторая специальная ассоциированная полугруппа), и это тесно связано с неединственностью решений уравнения $L_{A, b}^{*} \mu=0$ в классе вероятностных мер. С другой стороны, мы найдем условия, гарантирующие единственность ассоциированной полугруппы, причем единственность оказывается равносильной собственной инвариантности $\mu$ относительно специальной ассоциированной полугруппы в том случае, когда коэффициент сноса $b$ локально интегрируем по Лебегу в степени больше размерности пространства. В последнем случае полугруппа в самом деле порождается оператором $L_{A, b}$ в том классическом смысле, что замыкание оператора $\left(L_{A, b}, C_{0}^{\infty}\right)$ в $L^{1}(\mu)$ есть генератор полугруппы.

Напомним, что ограниченный оператор $T$ в $L^{1}(\mu)$ называется субмарковским, если $0 \leqslant T f \leqslant 1$ при $f \in L^{\infty}(\mu)$ и $0 \leqslant f \leqslant 1$. Если, кроме того, $T 1=1$, то $T$ называется марковским. В общем случае операторы $T_{t}^{\mu}$ построенной ниже ассоциированной полугруппы являются субмарковскими, но не марковскими. Кроме того, $\mu$ лишь субинвариантна для них, т. е.

$$
\int_{X} T_{t}^{\mu} f d \mu \leqslant \int_{X} f d \mu, \quad f \in L^{\infty}(\mu), \quad f \geqslant 0 .
$$


Заметим, что если $T$ - непрерывный оператор на $L^{1}(\mu)$ и переводит $L^{\infty}(\mu)$ в $L^{\infty}(\mu)$, то по интерполяционной теореме он переводит $L^{p}(\mu)$ в $L^{p}(\mu)$ для всех $p \in[1,+\infty)$, а его сопряженный оператор $T^{*}$ также переводит каждое $L^{p}(\mu)$ в $L^{p}(\mu)$, включая $p=1$.

Сначала мы обсудим случай $A=\mathrm{I}$, изученный в [7], где можно найти доказательства.

Теорема 1.5.1. Предположим, что $A=\mathrm{I},|b| \in L_{\mathrm{loc}}^{p}\left(\mathbb{R}^{d}\right)$ для некоторого $p>d \geqslant 2$ и $\mu$ - такая вероятностная мера на $\mathbb{R}^{d}$, что $L^{*} \mu=0$, где $L=L_{\mathrm{I}, b}$, причем функиия $\varrho:=d \mu / d x$ такова, что $|b-\nabla \varrho / \varrho| \in L^{1}(\mu)$. Тогда справедливы следующие утверждения.

(i) Существует ровно одна сильно непрерывная полугруппа $\left(T_{t}^{\mu}\right)_{t>0}$ в $L^{1}(\mu)$ такал, что ее генератор $\left(L_{\mu}, D\left(L_{\mu}\right)\right)$ продолжает $L$, m.е. $C_{0}^{\infty}\left(\mathbb{R}^{d}\right) \subset D\left(L_{\mu}\right)$ $u L_{\mu}=L$ на $C_{0}^{\infty}\left(\mathbb{R}^{d}\right)$. Кроме того, оператор $\left(L_{\mu}, D\left(L_{\mu}\right)\right)$ является замыканием onepamopa $\left(L, C_{0}^{\infty}\left(\mathbb{R}^{d}\right)\right)$ в $L^{1}(\mu)$.

(ii) Полугруппа $\left(T_{t}^{\mu}\right)_{t>0}$ является марковской.

(iii) Если $\left(G_{\alpha}\right)_{\alpha>0}$ - соответствующая резольвента, то $G_{\alpha} f$ имеет единственную непрерывную $\mu$-версию для всех $f \in L^{d p /(d+p)}(\mu)$ u всех $\alpha>0$. $B$ частности, $\left(G_{\alpha}\right)_{\alpha>0}$ является сильно феллеровской, т.е. $G_{\alpha} f$ имеет непрерывную $\mu$-версию для всех $f \in L^{\infty}(\mu), \alpha>0$.

(iv) Для всяких $f \in C_{0}^{\infty}\left(\mathbb{R}^{d}\right)$ u $t>0$ функиия $T_{t}^{\mu} f$ имеет единственную непрерывную $\mu$-версию $\widehat{T_{t}^{\mu}} f u \mu$-единственная вероятностная мера $\nu$ на $\mathbb{R}^{d}$, для которой

$$
\int \widetilde{T_{t}^{\mu}} f d \nu=\int f d \nu \quad \text { для всех } f \in C_{0}^{\infty}\left(\mathbb{R}^{d}\right) \text { и всех } t>0 .
$$

Теорема 1.5.2. Предположим, что вероятностная мера $\mu$ на $\mathbb{R}^{d}$ удовлетворяет уравнению $L^{*} \mu=0$, где $L:=L_{\mathrm{I}, b} u|b| \in L_{\mathrm{loc}}^{2}(\mu)$. Пусть $\varrho-n л о т-$ ность $\mu$.

(i) Предположим, что

$$
\left|b-\frac{\nabla \varrho}{\varrho}\right| \in L^{2}(\mu) .
$$

Тогда существует сильно непрерывная полугруппа $\left(T_{t}^{\mu}\right)_{t \geqslant 0}$ на $L^{1}(\mu)$ с генератором $\left(L_{\mu}, D\left(L_{\mu}\right)\right)$, продолжающим $L$. Более того, $\left(T_{t}^{\mu}\right)_{t \geqslant 0}$ является марковской и мера $\mu$ является $\left(T_{t}^{\mu}\right)_{t \geqslant 0}$-инвариантной.

(ii) Предположим, что $|b| \in L^{2}(\mu)$. Тогда $|b-\nabla \varrho / \varrho| \in L^{2}(\mu)$, поэтому верно заключение (i).

(iii) Предположим, что $|b| \in L_{\mathrm{loc}}^{p}\left(\mathbb{R}^{d}\right)$ для некоторого $p>d \geqslant 2$ и что

$$
\left|b-\frac{\nabla \varrho}{\varrho}\right| \in L^{q}(\mu) \quad \text { для некоторого } q \in[1, \infty] .
$$

Пусть $r:=2-2 /(q+1)$, где $1 / \infty:=0$. Тогда ограничение $\left(T_{t}^{\mu}\right)_{t \geqslant 0}$ на $L^{r}(\mu)$ является единственной сильно непрерывной полугруппой на $L^{r}(\mu)$, генератор которой продолжает $L$. Этот генератор является замыканием $\left(L, C_{0}^{\infty}\left(\mathbb{R}^{d}\right)\right)$ в $L^{r}(\mu)$. 
ПРЕДЛОЖЕНИЕ 1.5.3. Предположим, что $\mu_{1} u \mu_{2}-$ две вероятностные меры на $\mathbb{R}^{d}$, удовлетворяющие уравнению $L^{*} \mu=0$ с $L=L_{\mathrm{I}, b}$, причем $\mu_{2}$ абсолютно непрерывна относительно $\mu_{1}$ и функция $|b|$ является $\mu_{i}$-квадратично интегрируемой для обеих мер. Пусть $\left(T_{t}^{\mu_{i}}\right)_{t \geqslant 0}$ - соответствующие сильно непрерывные полугруппы на $L^{1}\left(\mu_{i}\right)$, генераторы $\left(L^{\mu_{i}}, D\left(L^{\mu_{i}}\right)\right)$ которых продолжают $L, i=1,2$, существующие по предыдущей теореме. Тогда $T_{t}^{\mu_{1}} f=T_{t}^{\mu_{2}} f$ $\mu_{1}$-n.в. для всех $t>0$ и всех ограниченных борелевских функций $f$ на $\mathbb{R}^{d}$.

Это предложение можно доказать с помощью модификации рассуждения из [49], основанного на формуле Дюамеля.

ЗАмечАниЕ 1.5.4. (i) Укажем, что все сильно непрерывные полугруппы $\left(T_{t}^{\mu}\right)_{t \geqslant 0}$, появляющиеся выше, автоматически оказываются сжатиями во всех $L^{p}(\mu)$, поскольку они субмарковские и мера $\mu$ является $T_{t}^{\mu}$-инвариантной.

(ii) В ситуации теоремы 1.5.2 (i) существует "хороший” марковский процесс в $\mathbb{R}^{d}$, переходные вероятности которого задаются полугруппой $\left(T_{t}^{\mu}\right)_{t \geqslant 0}$ и который является слабым решением стохастического уравнения $d \xi_{t}=d w_{t}+b\left(\xi_{t}\right) d t$. Это вытекает из работы [50], к которой мы отсылаем по поводу деталей и точных определений.

Рассмотрим симметричный случай, т.е. случай, когда оператор $L_{\mathrm{I}, b}$ симметричен; оказывается, это соответствует тому, что $b$ есть логарифмический градиент, см. пример 1.1.3 (более точное описание этого случая дано ниже).

ПредлОЖенИЕ 1.5.5. Пустъ $b=\nabla \varrho / \varrho$, где $\varrho \in W_{\mathrm{loc}}^{1,1}\left(\mathbb{R}^{d}\right)$ - вероятностная плотность, и пусть $\mu:=\varrho d x$. Тогда существует сильно непрерьвная марковская полугруппа $\left(T_{t}^{\mu}\right)_{t \geqslant 0}$ на $L^{1}(\mu)$, генератор $\left(L^{\mu}, D\left(L^{\mu}\right)\right)$ которой продолжает $L=L_{\mathrm{I}, b}$ и которая имеет $\mu$ в качестве $\left(T_{t}^{\mu}\right)_{t \geqslant 0}$-инвариантной мерь. Более того, ограничение $\left(T_{t}^{\mu}\right)_{t \geqslant 0}$ на $L^{2}(\mu)$ состоит из симметричных операторов.

ЗАмЕЧАнИЕ 1.5.6. (i) Единственность $\left(T_{t}^{\mu}\right)_{t \geqslant 0}$ в предложении 1.5.5 обеспечивается теоремой 1.5.2 (ii) при условии, что $|b| \in L^{2}(\mu)$. Однако, согласно [51; теорема 3.1], можно ослабить последнее условие до включения $|b| \in L_{\text {loc }}^{2}(\mu)$ и все еще иметь единственность $\left(T_{t}^{\mu}\right)_{t \geqslant 0}$ среди всех сильно непрерывных cuмметричных марковских полугрупп на $L^{2}(\mu)$, генераторы которых продолжают $L$ (но мы не знаем, существенно ли здесь условие симметричности). Отметим также, что в рассматриваемом симметричном случае можно взять $r=2$ в теореме 1.5.2 (iii), что было впервые установлено в [6; следствие 8]). Отметим, что без дополнительных предположений мера $\mu$ в этом предложении может не быть единственной вероятностной мерой, удовлетворяющей уравнению $L_{\mathrm{I}, b}^{*} \mu=0$, даже среди мер с логарифмическим градиентом $b$ (см. пример 1.6.1 ниже).

(ii) В ситуации предложения 1.5.5 из его доказательства и общей теории [17] вытекает, что существует диффузионный процесс в $\mathbb{R}^{d}$ с переходной полугруппой $\left(T_{t}^{\mu}\right)_{t \geqslant 0}$.

А.Н. Колмогоров в своей знаменитой работе [2] изучил следующую задачу. Пусть $\left(\xi_{t}\right)_{t \geqslant 0}$ - диффузия в конечномерном римановом многообразии $X$ (в колмогоровском случае оно было компактно) с генератором $L=(\Delta+b) / 2$, где $b$ - гладкое векторное поле на $X$. Когда процесс $\eta_{t}=\xi_{T-t}$ управляется тем же самым уравнением, т. е. имеет тот же самый генератор $L$ ? Ответ, найденный А.Н. Колмогоровым, гласит: если и только если $b$ есть градиент функции. 
Ранее этот вопрос был рассмотрен Э. Шрёдингером [52] в одномерном случае. В работе [2] А.Н. Колмогоров рассматривал только решения уравнений Фоккера-Планка как плотности переходных вероятностей; в то время еще не было развито стохастическое интегрирование (соответствующее стохастическое уравнение на $\mathbb{R}^{d}$ имело бы вид $\left.d \xi_{t}=d w_{t}+2^{-1} b\left(\xi_{t}\right) d t\right)$. Более того, в основном был рассмотрен случай компактного $X$, когда инвариантная вероятностная мера всегда существует. В этом случае свойство, изученное А. Н. Колмогоровым, равносильно симметризуемости $\left(\xi_{t}\right)_{t \geqslant 0}$. Таким образом, результат А. Н. Колмогорова есть критерий симметризуемости диффузии в компактном многообразии. В некомпактном случае требуются дополнительные условия, чтобы обеспечить существование инвариантных вероятностных мер (см. предыдущий пункт). Разницу между существованием инвариантных мер $\mu=\varrho d x$ и симметризуемостью диффузии $\left(\xi_{t}\right)_{t \geqslant 0}$ с генератором $L=(\Delta+b) / 2$ можно усмотреть из следствия 1.8.2 ниже, которое распространяет теорему А.Н. Колмогорова на общие сносы из $L^{2}(\mu)$. Согласно этому следствию, сносы симметризуемых диффузий в $\mathbb{R}^{d}$ являются логарифмическими градиентами мер (аналогичный факт верен и для многообразий, см. [24]). Если $|b| \in L_{\mathrm{loc}}^{p}\left(\mathbb{R}^{d}\right)$ при некотором $p>d$, то получаем точный аналог, поскольку $\mu$ имеет положительную плотность $\varrho$ и $b=\nabla \ln \varrho$, т. е. $b$ в самом деле есть градиент функции.

Теперь рассмотрим случай непостоянного $A$, изученный в [11], [12], где можно найти доказательства. Зафиксируем открытое подмножество $\Omega$ риманова многообразия $M$ размерности $d$. До конца этого пункта будем предполагать, что $A$ и $b$ удовлетворяют следующим условиям при некотором $p>d$ :

$$
\begin{aligned}
& \text { (A1) } a^{i j} \in C(\Omega) \cap W_{\mathrm{loc}}^{p, 1}(\Omega), \quad \operatorname{det} A(x) \neq 0, \\
& \text { (A2) } b^{i} \in L_{\mathrm{loc}}^{p}(\Omega) .
\end{aligned}
$$

Будем рассматривать меры $\mu \geqslant 0$ на $\Omega$, удовлетворяющие нашему уравнению $L_{A, b}^{*} \mu=0$; в некоторых результатах мы будем иметь дело с уравнением $\mathscr{L}_{A, b}^{*} \mu=0$. Пусть $\varrho-$ плотность $\mu$. Положим

$$
\beta_{\mu, A}:=\left(\beta_{\mu, A}^{i}\right)_{i=1}^{d}, \quad \beta_{\mu, A}^{i}:=\partial_{j} a^{i j}+a^{i j} \frac{\partial_{j} \varrho}{\varrho} .
$$

Тогда $\beta_{\mu, A}^{i} \in L_{\mathrm{loc}}^{p}(\Omega)$. Можно записать

$$
L_{A, b} \varphi=L_{A, \beta_{\mu, A}}+\left\langle b-\beta_{\mu, A}, \nabla \varphi\right\rangle, \quad \varphi \in C_{0}^{\infty}(\Omega) .
$$

В случае дивергентной формы вместо $\beta_{\mu, A}$ будет использовано отображение $A \beta_{\mu}$. Оператор $L_{A, \beta_{\mu, A}}$ симметричен на $L^{2}(\Omega, \mu)$, т. е.

$$
\int_{\Omega} L_{A, \beta_{\mu, A}} \varphi \psi d \mu=\int_{\Omega} \varphi L_{A, \beta_{\mu, A}} \psi d \mu \quad \forall \varphi, \psi \in C_{0}^{\infty}(\Omega) .
$$

В самом деле, по формуле интегрирования по частям обе части (1.5.3) равны

$$
-\int_{\Omega}\langle A \nabla \varphi, \nabla \psi\rangle d \mu
$$


Аналогично для операторов в дивергентной форме имеем

$$
\int_{\Omega} \psi \mathscr{L}_{A, A \beta_{\mu}} \varphi d \mu=\int_{\Omega} \varphi \mathscr{L}_{A, A \beta_{\mu}} \psi d \mu .
$$

Заметим, что если $A=\mathrm{I}$, то $b_{\mu, \mathrm{I}}=\beta_{\mu}$ и

$$
L_{\mathrm{I}, b} \varphi=\mathscr{L}_{\mathrm{I}, b} \varphi=\Delta \varphi+\langle b, \nabla \varphi\rangle .
$$

Если $\mu$ - неотрицательная мера на $\Omega$ с плотностью $\varrho \in W_{\mathrm{loc}}^{1,1}(\Omega), A$ и $b$ удовлетворяют условиям (A1) и (A2) из (1.5.2), причем $\beta_{\mu}$ и $\beta_{\mu, A}$ определены указанным выше образом, то равенство $L_{A, b}^{*} \mu=0$ равносильно тождеству

$$
\int_{\Omega}\left\langle b-\beta_{\mu, A}, \nabla \varphi\right\rangle d \mu=0, \quad \varphi \in C_{0}^{\infty}(\Omega)
$$

или в короткой записи

$$
\operatorname{div}_{\mu}\left(b-\beta_{\mu, A}\right)=0 .
$$

В самом деле, достаточно заметить, что по формуле интегрирования по частям имеем

$$
\int_{\Omega}\left[\partial_{j} a^{i j} \partial_{i} \varphi \varrho+a^{i j} \partial_{i} \varphi \partial_{j} \varrho\right] d x=-\int_{\Omega} a^{i j} \partial_{j} \partial_{i} \varphi \varrho d x .
$$

Аналог (1.5.4) для оператора дивергентного вида есть

$$
\int_{\Omega}\left\langle b-A \beta_{\mu}, \nabla \varphi\right\rangle d \mu=0 \quad \forall \varphi \in C_{0}^{\infty}(\Omega) .
$$

Векторное поле

$$
\hat{b}:=2 \beta_{\mu, A}-b
$$

называют дуальным сносом (в теории диффузионных процессов это есть снос процесса с обращенным временем). В случае дивергентной формы оператора дуальный снос задается равенством

$$
\tilde{b}:=2 A \beta_{\mu}-b
$$

Ясно, что

$$
L_{A, \hat{b}}=L_{A, \beta_{\mu, A}}-\left(b^{i}-\beta_{\mu, A}^{i}\right) \partial_{i} \quad \text { на } C_{0}^{\infty}(\Omega),
$$

и очевидным образом $L_{A, \hat{b}}$ является формально сопряженным для $L_{A, b}$, т. е.

$$
\int_{\Omega} \psi L_{A, b} \varphi d \mu=\int_{\Omega} \varphi L_{A, \hat{b}} \psi d \mu, \quad \varphi, \psi \in C_{0}^{\infty}(\Omega) .
$$

Аналогично,

$$
\int_{\Omega} \psi \mathscr{L}_{A, b} \varphi d \mu=\int_{\Omega} \varphi \mathscr{L}_{A, \tilde{b}} \psi d \mu, \quad \varphi, \psi \in C_{0}^{\infty}(\Omega) .
$$

Заметим, что в силу (1.5.3) и (1.5.5) мы имеем

$$
\mu \in \mathscr{M}_{\mathrm{ell}}^{A, \beta_{\mu, A}} \cap \mathscr{M}_{\mathrm{ell}}^{A, \hat{b}} .
$$


Tеорема 1.5.7. (i) Пусть $\Omega$ - открытое подмножество $\mathbb{R}^{d}$. Предположим, что выполнены условия (A1) и (A2) из (1.5.2). Пусть $\mu \in \mathscr{M}_{\mathrm{ell}}^{A, b}$. Тогда имеется замкнутое продолжение $\left(L_{A, b}^{\mu}, D\left(L_{A, b}^{\mu}\right)\right)$ оператора $\left(L_{A, b}, C_{0}^{\infty}(\Omega)\right)$, nорождающее субмарковскую сжимающую $C_{0}$-полугруппу $\left(T_{t}^{\mu}\right)_{t \geqslant 0}$ на $L^{1}(\Omega, \mu)$ со следующими свойствами:

(а) указанное выше естественное продолжение сопряженной полугруппы на $L^{1}(\Omega, \mu)$ имеет генератор $L_{A, \hat{b}}^{\mu}$, совпадающий с $L_{A, \hat{b}}$ на $C_{0}^{\infty}(\Omega)$;

(b) для всякой ограниченной измеримой функции $f$ с компактным носителем функиия $\left(I-L_{A, b}^{\mu}\right)^{-1} f$ есть предел в $L^{1}(\Omega, \mu)$ функиий $u_{n}$, являюшихся решениями задач Дирихле $\left(I-L_{A, b}\right) u_{n}=f$ с нулевыми граничными условиями на областях $B_{n}$ с компактными замыканиями $\bar{B}_{n} \subset B_{n+1}$ и гладкими границами $\partial B_{n}$, для которых $\Omega=\bigcup_{n=1}^{\infty} B_{n}$; кроме того, н субинвариантна для $\left(T_{t}^{\mu}\right)_{t \geqslant 0}$.

(ii) Это же верно, если $\Omega$ - открытое подмножество полного риманова многообразия $M$ и оператор $L_{A, b}$ заменен на оператор $\mathscr{L}_{A, b}$ вида

$$
\mathscr{L}_{A, b} \varphi=\operatorname{div}(A \nabla \varphi)+\langle b, \nabla \varphi\rangle, \quad \varphi \in C_{0}^{\infty}(\Omega),
$$

где $b$ - векторное поле на $M u A(x)$ - положительный оператор на $T_{x} M$, для которого условия из (i) выполнены в локальных координатах.

(iii) В предположениях (i) или (ii) полугруппа $\left(T_{t}^{\mu}\right)_{t \geqslant 0}$ имеет следующее свойство: для каждого $\psi \in C_{0}^{\infty}(\Omega)$ и каждого $t \geqslant 0$ функиия $T_{t}^{\mu} \psi$ обладает непрерывной модификацией $\widehat{T_{t}^{\mu} \psi}$ такой, что для каждого компактного множества $K \subset \Omega$ имеем $\lim _{t \rightarrow 0} \widetilde{T_{t}^{\mu} \psi}(x)=\psi(x)$ равномерно по $x \in K$.

Полугруппа $\left(T_{t}^{\mu}\right)_{t \geqslant 0}$, построенная в этой теореме, будет играть ниже очень важную роль; в общем случае она не является единственной полугруппой, ассоциированной с $L_{A, b}$ в смысле, объясненном в начале этого пункта, но она единственна среди ассоциированных полугрупп со свойством (b). Ниже индекс $\mu$ в $T_{t}^{\mu}$ будет указывать эту конкретную полугруппу. Такая полугруппа существует и при более слабых условиях на $A$ и $b$ (см. [50]).

ЗАмЕчАниЕ 1.5.8. В утверждении (i) теоремы выше ввиду включения $\mu \in$ $\mathscr{M}_{\mathrm{ell}}^{A, \hat{b}}$ также "формально сопряженный" оператор $\left(L_{A, \hat{b}}, C_{0}^{\infty}(\Omega)\right)$ для оператоpa $\left(L_{A, b}, C_{0}^{\infty}(\Omega)\right)$ имеет замкнутое продолжение $\left(L_{A, \hat{b}}^{\mu}, D\left(L_{A, \hat{b}}^{\mu}\right)\right)$, порождающее субмарковскую $C_{0}$-полугруппу $\left(\widehat{T}_{t}^{\mu}\right)_{t \geqslant 0}$ на $L^{1}(\Omega, \mu)$. Согласно [50; замечание 1.7 (ii)], мы имеем

$$
\int_{\Omega} g T_{t}^{\mu} f d \mu=\int_{\Omega} f \widehat{T}_{t}^{\mu} g d \mu, \quad f, g \in L^{\infty}(\Omega, \mu) .
$$

Такое же соотношение выполнено и для соответствующих резольвент $\left(G_{\alpha}^{\mu}\right)_{\alpha>0}$ и $\left(\widehat{G}_{\alpha}^{\mu}\right)_{\alpha>0}$. Равенство $(1.5 .7)$, в частности, немедленно показывает, что мера $\mu$ является $\left(T_{t}^{\mu}\right)_{t \geqslant 0}$-инвариантной, если и только если для каждого $t \geqslant 0$ мы имеем $\widehat{T}_{t}^{\mu} 1=1$. Значит, поскольку обе полугруппы субмарковские, это выполнено в точности тогда, когда $T_{t}^{\mu} 1=1$ для всех $t \geqslant 0$, что в свою очередь равносильно инвариантности $\mu$ относительно $\left(\widehat{T}_{t}^{\mu}\right)_{t \geqslant 0}$. Равенство $T_{t}^{\mu} 1=1$ равносильно 
включению $1 \in D\left(L_{A, b}^{\mu}\right)$ (или $1 \in D\left(L_{A, \hat{b}}^{\mu}\right)$, не всегда выполненному, несмотря на равенство $L_{A, b} 1=L_{A, \hat{b}} 1=0$.

Стоит отметить, что для симметричного оператора $L_{A, b}$ (или $\mathscr{L}_{A, b}$ ) замкнутое продолжение, упомянутое в теореме, является расширением Фридрихса. Условие симметричности полугруппы $\left(T_{t}^{\mu}\right)_{t \geqslant 0}$ видно из (1.5.6). Так называемое обращение времени для конечномерных и бесконечномерных диффузий (т. е. процесс $\xi_{T-t}$ для данной диффузии $\xi_{t}$ ) обсуждается в [53]-[56].

Нам неизвестно, единственна ли субмарковская сильно непрерывная полугруппа, генератор которой продолжает $L_{A, b}$ (т.е. выполнено ли свойство (b) в (i) автоматически). Как мы увидим ниже, это верно при некоторых дополнительных предположениях.

Напомним, что оператор $L$ на плотной области определения $\mathscr{D}$ в банаховом пространстве $X$ называется диссипативным, если для каждого $u \in \mathscr{D}$ существует такой функционал $l_{u} \in X^{*}$, что $\left\|l_{u}\right\|_{X^{*}}=\|u\|_{X}, l_{u}(u)=\|u\|_{X}^{2}$ и $l_{u}(L u) \leqslant 0$. Диссипативный оператор $L$ называется существенно $m$-диссипативным, если он удовлетворяет следующему дополнительному условию:

$$
\overline{(L-\lambda I)(\mathscr{D})}=X \quad \forall \lambda>0,
$$

где $\bar{E}$ обозначает замыкание $E$. На самом деле достаточно, чтобы это условие было выполнено для некоторого $\lambda>0$.

Лемма 1.5.9. Пусть мера $\mu \geqslant 0$ удовлетворяют уравнению $L_{A, b}^{*} \mu=0$ с коэффичиентами, удовлетворяющими условиям (A1) и (А2) из (1.5.2). Тогда оператор $L_{A, b}$ диссипативен на области определения $C_{0}^{\infty}(\Omega)$ в пространстве $L^{1}(\Omega, \mu)$. В частности, он замыкаем. Это же верно для оператора $\mathscr{L}_{A, b}$ в дивергентной форме в случае многообразия.

Значит, $L_{A, b}$ существенно $m$-диссипативен на области определения $C_{0}^{\infty}(\Omega)$ в $L^{1}(\Omega, \mu)$, если и только если множество $\left(L_{A, b}-\lambda I\right)\left(C_{0}^{\infty}(\Omega)\right)$ плотно в $L^{1}(\Omega, \mu)$ для некоторого (тогда для каждого) $\lambda>0$. В этом случае оператор $\left(L_{A, b}\right.$, $\left.C_{0}^{\infty}(\Omega)\right)$ называется $L^{1}(\Omega, \mu)$-единственным; см. п. 1.7 про достаточные условия для этого.

Обозначим через $\left(\bar{L}_{A, b}^{\mu}, D\left(\bar{L}_{A, b}^{\mu}\right)\right)$ замыкание оператора $\left(L_{A, b}, C_{0}^{\infty}(\Omega)\right)$ в пространстве $L^{1}(\Omega, \mu)$. Замыкание $\left(\overline{\mathscr{L}}_{A, b}^{\mu}, D\left(\overline{\mathscr{L}}_{A, b}^{\mu}\right)\right)$ оператора $\mathscr{L}_{A, b}$ определяется аналогично.

ПреДЛОЖЕНИЕ 1.5.10. Пусть $\mu \in \mathscr{M}_{\mathrm{ell}}^{A, b}$, где $A$ u b удовлетворяют условиям (A1) и (A2) из (1.5.2). Следующие утверждения равносильны:

(i) $\left(\bar{L}_{A, b}^{\mu}, D\left(\bar{L}_{A, b}^{\mu}\right)\right)$ порождает $C_{0}$-полугруппу $\left(T_{t}\right)_{t \geqslant 0}$, m. е. силъно непреръвную полугруппу ограниченных операторов $T_{t}$ в $L^{1}(\Omega, \mu)$;

(ii) для некоторого (тогда и для всякого) $\lambda>0$ множество

$$
\left(L_{A, b}-\lambda I\right)\left(C_{0}^{\infty}(\Omega)\right)
$$

плотно в $L^{1}(\Omega, \mu)$ (что равносильно тому, что оператор $\left(L_{A, b}, C_{0}^{\infty}(\Omega)\right.$ ) существенно т-диссипативен в $\left.L^{1}(\Omega, \mu)\right)$;

(iii) существует ровно одна $C_{0}$-полугруппа на $L^{1}(\Omega, \mu)$, генератор которой продолжает оператор $\left(L_{A, b}, C_{0}^{\infty}(\Omega)\right)$. 
Если какое-то (а тогда каждое) из утверждений (i)-(iii) верно, то полугруппь $\left(T_{t}^{\mu}\right)_{t \geqslant 0} u\left(\widehat{T}_{t}^{\mu}\right)_{t \geqslant 0}$ являются марковскими и мера $\mu$ инвариантна для них. Наконец, это же верно для оператора $\mathscr{L}_{A, b}$ в дивергентной форме.

ЗАмЕчАнИЕ 1.5.11. Для ограниченной области $\Omega$ (с гладкой границей) утверждение (iii) (значит, также (i) и (ii)) в предложении 1.5.10 неверны, даже если $A=\mathrm{I}, b \equiv 0$. Поэтому мы будем в основном интересоваться случаем $\Omega=M$. Как мы увидим ниже, утверждения (i)-(iii) равносильны инвариантности $\mu$ относительно полугруппы $\left(T_{t}^{\mu}\right)_{t \geqslant 0}$.

Ниже мы увидим, что оператор $L_{A, b}$ на $C_{0}^{\infty}(\Omega)$ может иметь различные замкнутые расширения (даже порождающие сильно непрерывные полугруппы). Значит, его замыкание может не порождать сильно непрерывных полугрупп (т. е. может не быть генератором). Это случается, даже если $A=\mathrm{I}$ и $b$ бесконечно дифференцируемо.

Следующая теорема дает полезную информацию об области определения генератора $\left(T_{t}^{\mu}\right)_{t \geqslant 0}$ в $L^{p}(\Omega, \mu)$. Известно, что для каждого $r \in[1, \infty)$ ограничение $\left(T_{t}^{\mu}\right)_{t \geqslant 0}$ на $L^{r}(\Omega, \mu)$ является сильно непрерывной полугруппой в $L^{r}(\Omega, \mu)$. Ее генератор обозначим через $\left(L_{A, b}^{\mu, r}, D\left(L_{A, b}^{\mu, r}\right)\right)$. Нетрудно проверить, что

$$
D\left(L_{A, b}^{\mu, r}\right)=\left\{f \in D\left(L_{A, b}^{\mu}\right) \cap L^{r}(\Omega, \mu): L_{A, b}^{\mu} f \in L^{r}(\Omega, \mu)\right\} .
$$

Tеорема 1.5.12. (i) B ситуации теоремы 1.5 .7 имеем

$$
\left(L_{A, b}^{\mu, p}, D\left(L_{A, b}^{\mu, p}\right)\right) \subset\left\{f \in L^{p}(\Omega, \mu) \cap H_{\mathrm{loc}}^{p, 2}(\Omega): L_{A, b} f \in L^{p}(\Omega, \mu)\right\}
$$

$u L_{A, b}^{\mu, p} f=L_{A, b} f$ для всех $f \in D\left(L_{A, b}^{\mu, p}\right)$. Это же верно для всякого продолжения $(L, D(L))$ оператора $\left(L_{A, b}, C_{0}^{\infty}(\Omega)\right)$ со следуюшим свойством: он является генератором сильно непрерывной субмарковской полугруппы $\left(T_{t}\right)_{t \geqslant 0}$ на $L^{1}(\Omega, \mu)$, для которой сопряженная полугруппа $\left(T_{t}^{\prime}\right)_{t \geqslant 0}$ в $L^{p^{\prime}}(\Omega, \mu)$ (которая определена в результате продолжения $\left(T_{t}\right)_{t \geqslant 0}$ на $L^{p}(\Omega, \mu)$ как обгяснено выше) имеет генератор, совпадающий с $L_{A, \hat{b}}$ на $C_{0}^{\infty}(\Omega)$. Если выполнено равенство в (1.5.8), то н инвариантна для $\left(T_{t}^{\mu}\right)_{t \geqslant 0}\left(\right.$ равносильным образом, $\left.T_{t}^{\mu} 1=1\right)$.

(ii) Eсли оператор $\left(L_{A, \hat{b}}, C_{0}^{\infty}(\Omega)\right)$ существенно m-диссипативен в пространcтве $L^{p^{\prime}}(\Omega, \mu)$, m.е. множество $\left(L_{A, \hat{b}}-\lambda I\right)\left(C_{0}^{\infty}(\Omega)\right)$ плотно в $L^{p^{\prime}}(\Omega, \mu)$ для некоторого $\lambda>0$, то выполнено равенство в (1.5.8).

Наконеи, те же самые утверждения верны для $\mathscr{L}_{A, b}$.

Следует предупредить читателя, что теорема 1.5.7 не утверждает, что субмарковская полугруппа, генератор которой продолжает $L_{A, b}$, единственна: мы не знаем, верно ли это при указанных предположениях, а без свойства субмарковости (т. е. с единственным требованием сильной непрерывности) это в общем случае неверно. По этой причине мы всегда выделяем полугруппу $\left(T_{t}^{\mu}\right)_{t \geqslant 0}$. Кроме того, мера $\mu$ может быть лишь субинвариантной для полугруппы и не инвариантной. Мы уже знаем, что эти два явления тесно связаны: предложение 1.5.10 показывает, что единственность ассоциированной сильно непрерывной полугруппы равносильна существенной $m$-диссипативности $L_{A, b}$.

Введем следующее подмножество множества $\mathscr{M}_{\mathrm{ell}}^{A, b}$ :

$$
\mathscr{M}_{\mathrm{ell}, \mathrm{md}}^{A, b}:=\left\{\mu \in \mathscr{M}_{\mathrm{ell}}^{A, b}: \overline{\left(L_{A, b}-I\right)\left(C_{0}^{\infty}(\Omega)\right)}=L^{1}(\Omega, \mu)\right\} .
$$

То же обозначение будет использоваться для оператора $\mathscr{L}_{A, b}$. 
Следующий результат из [12] дает удобную техническую характеризацию существенной $m$-диссипативности $L_{A, b}$ и показывает, что она равносильна инвариантности $\mu$ относительно ассоциированной полугруппы $\left(T_{t}^{\mu}\right)_{t \geqslant 0}$ из теоремы 1.5.7. В случае $\Omega=\mathbb{R}^{d}$ этот результат был доказан в [50; предложение 1.9] при более общих предположениях относительно $A, b$ и $\mu$ (выполнение этих предположений в нашем случае вытекает из обсуждавшихся в п. 1.2 результатов).

ТЕОРема 1.5.13. Предположим, что выполнены условия (A1) и (А2) из (1.5.2). Пусть $\mu \in \mathscr{M}_{\mathrm{ell}}^{A, b}$. Тогда следующие утверждения равносильны:

(i) $\mu \in \mathscr{M}_{\text {ell,md }}^{A, b}$;

(ii) $\mu$ инвариантна для $\left(T_{t}^{\mu}\right)_{t \geqslant 0}$;

(iii) существуют такие функиии $\chi_{n} \in W_{\mathrm{loc}}^{2,1}(\Omega, \mu)$ и $\alpha>0$, что $\left(1-\chi_{n}\right)^{+} \epsilon$ $L^{\infty}(\Omega, \mu) u\left(1-\chi_{n}\right)^{+}=0$ вне некоторых компактов, $\lim _{n \rightarrow \infty} \chi_{n}(x)=0 \mu$-n.в., причем для $\eta=1$ или $\eta=-1$ мы имеем

$$
\int\left\langle A \nabla \chi_{n}, \nabla \varphi\right\rangle d \mu+\alpha \int \chi_{n} \varphi d \mu+\eta \int\left\langle b-\beta_{\mu, A}, \nabla \chi_{n}\right\rangle \varphi d \mu \geqslant 0
$$

для всех неотрицательных $\varphi \in C_{0}^{\infty}(\Omega)$ и всех $n \in \mathbb{N} ;$ соответствующее условие в случае $\mathscr{L}_{A, b}$ имеет вид

$$
\int\left\langle A \nabla \chi_{n}, \nabla \varphi\right\rangle d \mu+\alpha \int \chi_{n} \varphi d \mu+\eta \int\left\langle b-A \beta_{\mu}, \nabla \chi_{n}\right\rangle \varphi d \mu \geqslant 0 ;
$$

(iv) $\mu \in \mathscr{M}_{\mathrm{ell}, \mathrm{md}}^{A, \hat{b}}$ (соответственно $\mu \in \mathscr{M}_{\mathrm{ell}, \mathrm{md}}^{A, \tilde{b}}$ в случае $\mathscr{L}_{A, b}$ ).

ЗАмечАние 1.5.14. (i) Оператор $L_{A, b}$ не является существенно $m$-диссипативным на области $C_{0}^{\infty}(\Omega)$ в $L^{p}(\Omega, \mu)$ для некоторого $p \in[1, \infty)$ в точности тогда, когда существует такая ненулевая функция $h \in L^{p^{\prime}}(\Omega, \mu)$, где $p^{\prime}=p /(p-1)$, что мера $h \cdot \mu$ удовлетворяет уравнению $\left(L_{A, b}-1\right)^{*}(h \cdot \mu)=0$. Из доказательства теоремы 1.5.12 вытекает, что это равносильно (при наших предположениях относительно $A$ и $b$, конечно) следующему: существует такая ненулевая функция $h \in L^{p^{\prime}}(\Omega, \mu) \cap W_{\text {loc }}^{p, 2}(\Omega)$, что $L_{A, \hat{b}} h=h$ п. в.

(ii) Доказательство этой теоремы использует некоторые свойства нашей особой полугруппы $\left(T_{t}^{\mu}\right)_{t \geqslant 0}$, и мы не знаем, можно ли в утверждении (ii) использовать произвольную ассоциированную полугруппу.

Наша следующая цель - изучение связей между инфинитезимальной инвариантностью и инвариантностью. Как мы увидим, эти два понятия различны, но при некоторых дополнительных предположениях они совпадают. Сначала мы упомянем полезный технический результат, который показывает, что всякая разумная инвариантная мера $\nu$ полугруппы $\left(T_{t}^{\mu}\right)_{t \geqslant 0}$ на $L^{1}(\Omega, \mu)$, ассоциированной указанным в теореме 1.5.7 способом с вероятностной мерой $\mu$, удовлетворяющей уравнению $L_{A, b}^{*} \mu=0$, также удовлетворяет этому уравнению.

ПрЕДЛОЖЕНИЕ 1.5.15. Пусть $\Omega$ - открытое множество в $M$. Предположим, что выполнены условия (A1) $и$ (A2) из (1.5.2) $и \mu \in \mathscr{M}_{\mathrm{ell}}^{A, b}$. Пусть $\left(T_{t}^{\mu}\right)_{t \geqslant 0}$ - полугруппа, описанная в теореме 1.5.7. Предположим, что $\nu$ - такая вероятностная мера на $\Omega$, что $\nu \ll \mu u$

$$
\int T_{t}^{\mu} f d \nu=\int f d \nu \quad \forall f \in C_{0}^{\infty}(\Omega), \forall t>0 .
$$


Предположим, кроме того, что $b^{i} \in L_{\mathrm{loc}}^{q}(\Omega, \nu)$ для некоторого $q>1$. Тогда $\nu \in \mathscr{M}_{\mathrm{ell}}^{A, b}$.

В частности, если функиия $|b|$ локально ограничена, то всякая абсолютно непрерывная мера $\nu$, инвариантная для $\left(T_{t}^{\mu}\right)_{t \geqslant 0}$, удовлетворяет нашему эллиптическому уравнению и поэтому имеет положительную непрерывную плотность и эквивалентна $\mu$.

Следует отметить, что это предложение неверно без условия, что $\nu \ll \mu$. В самом деле, пусть $x_{0} \in \Omega$ фиксировано. Рассмотрим такие версии $T_{t}^{\mu} f$, где $f \in C_{0}^{\infty}(\Omega)$, что $T_{t}^{\mu} f\left(x_{0}\right)=f\left(x_{0}\right)$. Тогда дираковская мера в $x_{0}$ инвариантна для $T_{t}^{\mu}$. Однако мы не знаем, сколь существенно предположение, что $b^{i} \in L^{q}(\Omega, \nu)$. Ниже будет указано, что в случае, когда $p>d+2$, существуют единственным образом определенные субвероятностные ядра $K_{t}(\cdot, \cdot)$, для которых $K_{t} f$ является версией $T_{t}^{\mu} f$ для каждого $f \in L^{1}(\Omega, \mu)$, причем утверждение этого предложения верно для всякой инвариантной меры $\nu$ полугруппы $\left(K_{t}\right)_{t \geqslant 0}$.

Следующий результат, немного усиливающий [7; предложение 2.6 (ii)], дает достаточное условие, чтобы решение эллиптического уравнения было инвариантной мерой для нашей специальной ассоциированной полугруппы.

ПрЕДЛОЖЕНИЕ 1.5.16. Пусть $\mu \in \mathscr{M}_{\mathrm{ell}, \mathrm{md}}^{A, b} u\left(T_{t}^{\mu}\right)_{t \geqslant 0}-$ соответствующая

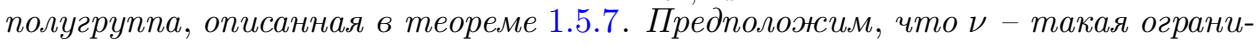
ченная борелевская мера на $\Omega$, что $L_{A, b}^{*} \nu=0$ и функи,я $\varrho:=d \nu / d \mu$ ограничена. Тогда мера $\nu$ является $\left(T_{t}^{\mu}\right)_{t \geqslant 0}$-инвариантной. Это же верно в случае $\mathscr{L}_{A, b}$.

Критерий инвариантности инфинитезимально инвариантной меры в терминах мартингальных задач получен в [57].

Следующее предложение показывает, что полугруппа $\left(T_{t}^{\mu}\right)_{t \geqslant 0}$ не может иметь инвариантных мер с положительной плотностью, если сама $\mu$ не инвариантна.

ПРЕДЛОЖЕНИЕ 1.5.17. Предположим, что $T$ - субмарковский оператор $в$ $L^{1}(\Omega, \mu)$, где $\mu$ - вероятностная мера на пространстве $\Omega$, являющаяся субинвариантной относительно T. Пусть $\nu$ - вероятностная мера на $\Omega$, эквивалентная $\mu$. Если мера $\nu$ инвариантна для $T$, то н также инвариантна.

ЗАмЕчаниЕ 1.5.18. Применительно к $\left(T_{t}^{\mu}\right)_{t \geqslant 0}$ в случае локального ограниченного $|b|$ этот результат (вместе с предложением 1.5.15 и с учетом субинвариантности $\mu$ относительно $\left.\left(T_{t}^{\mu}\right)_{t \geqslant 0}\right)$ показывает, что если $\mu$ не является инвариантной для $\left(T_{t}^{\mu}\right)_{t \geqslant 0}$, то никакая мера, эквивалентная $\mu$, не может быть инвариантной для $\left(T_{t}^{\mu}\right)_{t \geqslant 0}$. В п. 1.7 мы вернемся к этому вопросу.

Родственные результаты см. также в [58], [59]. О существовании инвариантных мер конечномерных диффузий см. [43], [60]-[63]. Общие проблемы, относящиеся к эргодичности диффузий и сходимости к инвариантным мерам, рассматриваются в [47], [63]-[74]. Свойства диффузионных полугрупп в $L^{p}$-пространствах относительно инвариантных мер и родственные задачи для эллиптических операторов изучаются в [75]-[78]. Гиперконтрактивность диффузионных полугрупп на конечномерных пространствах, неравенства Пуанкаре, логарифмические соболевские и другие близкие неравенства для них исследуются в [79]-[83]. В этих работах можно найти дополнительные ссылки. 
1.6. О неединственности решений. $\mathrm{K}$ проблеме единственности для решений эллиптических уравнений в классе всех вероятностных мер на всем пространстве мы обратимся в следующем пункте. Здесь же будут представлены некоторые отрицательные результаты в случае $A=\mathrm{I}$ и бесконечно дифференцируемого $b$. Легко построить такие примеры для сингулярных сносов $b$.

ПримеР 1.6.1. Пусть $\varrho$ - гладкая вероятностная плотность на вещественной прямой, причем $\varrho(0)=0$ и $\varrho(x)>0$, если $x \neq 0$. Пусть $b=\nabla \varrho / \varrho$ вне нуля и $b(0)=0$. Тогда вероятностная мера $\varrho d x$ удовлетворяет уравнению $L_{1, b}^{*} \mu=0$, но это уравнение имеет и другое решение $c \varrho I_{[0,+\infty)} d x$, где $c$ - нормирующая постоянная. Функция $\varrho^{\prime} / \varrho$ служит логарифмическим градиентом и этого второго решения, ибо $(-\infty, 0]$ имеет меру нуль относительно него.

В размерности 1 сингулярность $b$ - единственная причина неединственности.

ПРЕДЛОЖЕНИЕ 1.6.2. Предположим, что функиия b локалъно интегрируема по Лебегу на прямой. Тогда уравнение $L_{1, b}^{*} \mu=0$ может иметь самое большее одно решение в классе вероятностных мер.

ДокАЗАТЕЛьСтво. Согласно предложению 1.1.2, всякое решение задается плотностью

$$
\left(k_{1}+k_{2} \int_{0}^{x} \frac{1}{\psi(s)} d s\right) \psi(x), \quad \psi(x):=\int_{0}^{x} b(t) d t .
$$

Предположим, что имеется два линейно независимых решения. Значит, есть два линейно независимых вектора $\left(k_{1}, k_{2}\right)$, для которых соответствующая плотность интегрируема по всей прямой. Тогда из формул выше легко усмотреть, что $\psi \in L^{1}\left(\mathbb{R}^{1}\right)$. Следовательно, функция $1 / \psi$ неинтегрируема на $(-\infty, 0]$ и $[0,+\infty)$. Поскольку $1 / \psi>0$, то неопределенный интеграл от $1 / \psi$ стремится к $+\infty$ при $x \rightarrow+\infty$ и к $-\infty$ при $x \rightarrow-\infty$. Это показывает, что для неотрицательных решений мы должны иметь $k_{2}=0$, поэтому есть самое большее одно решение в классе вероятностных мер. Предложение доказано.

Однако во всякой размерности $d>1$ есть примеры неединственности с гладким $b$. Такие примеры найти нелегко, ибо, как мы увидим в следующем пункте, представленные выше теоремы существования всегда дают единственные решения. В течение нескольких лет проблема была открыта, пока в [11], [12] не был указан следующий простой пример.

ПРИМЕР 1.6.3. Пусть

$$
b^{i}(x)=-x_{i}-2 x_{\sigma(i)} e^{\left(x_{i}^{2}-x_{\sigma(i)}^{2}\right) / 2},
$$

где $\sigma:\{1,2,3, \ldots, d\} \rightarrow\{1,2,3, \ldots, d\}$ взаимно однозначно и $\sigma(i) \neq i$. Тогда уравнение имеет по крайней мере два решения: одно - стандартная гауссовская мера $\mu$ на $\mathbb{R}^{d}$ и другое - мера $\nu=v \cdot \mu \mathrm{c}$

$$
v(x)=c_{d} \sum_{i=1}^{d} \int_{-\infty}^{x_{i}} e^{-s^{2} / 2} d s,
$$

где $c_{d}$ - нормирующая постоянная. 
Более общим образом, пусть функция $f \in C^{2}\left(\mathbb{R}^{2}\right)$ ограничена, причем $f, f^{\prime}>0$ и $f^{\prime}, f^{\prime \prime} \in L^{1}\left(\mathbb{R}^{1}\right)$. Зададим $b=\left(b^{i}\right): \mathbb{R}^{d} \rightarrow \mathbb{R}^{d}$ посредством

$$
b^{i}(x):=\frac{f^{\prime \prime}\left(x_{i}\right)}{f^{\prime}\left(x_{i}\right)}+2 \frac{f^{\prime \prime}\left(x_{\sigma(i)}\right)}{f^{\prime}\left(x_{i}\right)}, \quad x=\left(x_{1}, \ldots, x_{d}\right) \in \mathbb{R}^{d},
$$

и положим

$$
\mu:=c_{1} \prod_{i=1}^{d} f^{\prime}\left(x_{i}\right) d x, \quad \nu:=c_{2} \sum_{i=1}^{d} f\left(x_{i}\right) \mu(d x),
$$

где $c_{1}, c_{2}>0$ - нормирующие константы. Тогда $\mu$ и $\nu$ - разные элементы в множестве $\mathscr{M}_{\mathrm{ell}}^{\mathrm{I}, b}$.

Однако даже в этом явном примере оставалось неизвестным, есть ли другие линейно независимые вероятностные решения. Явление неединственности было исследовано С. В. Шапошниковым [84], [85], получившим следующие результаты.

До конца этого пункта предполагается, что $A=\mathrm{I}$ и $b^{i} \in C^{\infty}\left(\mathbb{R}^{d}\right)$ при $1 \leqslant$ $i \leqslant d$. Тогда всякое решение уравнения $L_{\mathrm{I}, b}^{*} \mu=0$ имеет плотность $\varrho \in C^{\infty}\left(\mathbb{R}^{d}\right)$ и само уравнение может быть записано как следующее уравнение на $\varrho$ :

$$
\operatorname{div}(\nabla \varrho-b \varrho)=0 .
$$

Положим

$$
L:=L_{\mathrm{I}, b}, \quad a:=b \varrho-\nabla \varrho .
$$

Тогда $a \in C^{\infty}\left(\mathbb{R}^{d}, \mathbb{R}^{d}\right)$ и

$$
\operatorname{div} a=0 \text {. }
$$

Если $\varrho$ - вероятностная плотность, то, как мы знаем, $\varrho>0$, значит, коэффициент $b$ выражается следующим образом:

$$
b=\frac{\nabla \varrho}{\varrho}+\frac{a}{\varrho} .
$$

Будем искать другое решение уравнения $L^{*} \mu=0$ в виде $\nu=v \cdot \mu$. Мера $\nu=v \cdot \mu$ удовлетворяет тому же уравнению, если и только если функция $v$ удовлетворяет уравнению

$$
L_{\mu} v:=\operatorname{div}(\varrho \nabla v-a v)=0 .
$$

Конечно, каждая постоянная будет решением уравнения (1.6.2). Мы хотели бы найти достаточные условия существования ограниченного положительного решения, которое не постоянно. По аналогии с [86] введем следующую билинейную кососимметричную форму на $C_{0}^{\infty}\left(\mathbb{R}^{d}\right)$ :

$$
[f, g]:=\int_{\mathbb{R}^{d}}\langle a, \nabla f\rangle g d x
$$

Следующая теорема дает достаточные условия существования ограниченного положительного решения (1.6.2), которое не постоянно. 
ТЕОРема 1.6.4. Предположим, что есть такая функиия $\varphi \in C_{b}^{2}\left(\mathbb{R}^{d}\right)$, что $\langle a, \nabla \varphi\rangle \in L^{1}\left(\mathbb{R}^{d}\right)$,

$$
[\varphi, 1]=0 \quad u \quad[\varphi, \varphi]<0 .
$$

Тогда уравнение (1.6.2) имеет ограниченное положительное решение, не являющееся постоянной.

Ясно, что, умножая $v$ на положительную постоянную, мы получим вероятностную меру $v \cdot \mu$, удовлетворяющую уравнению $L^{*}(v \cdot \mu)=0$.

ЗАмЕчАНИЕ 1.6.5. Для проверки условий теоремы 1.6.4 полезно иметь в виду следующие выражения для $[\varphi, \varphi]$ и $[\varphi, 1]$. Пусть $\Omega_{n}-$ возрастающие области с кусочно-гладкими границами и $\mathbb{R}^{d}=\bigcup_{n=1}^{\infty} \Omega_{n}$. Поскольку $\operatorname{div} a=0$, мы имеем

$$
\begin{aligned}
& {[\varphi, \varphi]=\int_{\mathbb{R}^{d}}\langle a, \nabla \varphi\rangle \varphi d x=\lim _{n \rightarrow \infty} \int_{\Omega_{n}}\langle a, \nabla \varphi\rangle \varphi d x=\lim _{n \rightarrow \infty} \frac{1}{2} \int_{\partial \Omega_{n}}\left\langle a, \nu_{n}\right\rangle \varphi^{2} d s,} \\
& {[\varphi, 1]=\int_{\mathbb{R}^{d}}\langle a, \nabla \varphi\rangle d x=\lim _{n \rightarrow \infty} \int_{\Omega_{n}}\langle a, \nabla \varphi\rangle d x=\lim _{n \rightarrow \infty} \int_{\partial \Omega_{n}}\left\langle a, \nu_{n}\right\rangle \varphi d s,}
\end{aligned}
$$

где $\nu_{n}$ - внешняя нормаль на $\partial \Omega_{n}$. Поэтому для получения (1.6.3) достаточно доказать, что

$$
\lim _{n \rightarrow \infty} \int_{\partial \Omega_{n}}\left\langle a, \nu_{n}\right\rangle \varphi^{2} d s<0, \quad \lim _{n \rightarrow \infty} \int_{\partial \Omega_{n}}\left\langle a, \nu_{n}\right\rangle \varphi d s=0 .
$$

Чтобы дать пример уравнения $L^{*} \mu=0$, имеющего по крайней мере два различных вероятностных решения, достаточно сделать следующее. Прежде всего, находим гладкое векторное поле $a$, для которого $\operatorname{div} a=0$, и функцию $\varphi$, удовлетворяющую условиям теоремы 1.6.4. Затем фиксируем произвольную бесконечно дифференцируемую положительную функцию $\varrho$, для которой $\|\varrho\|_{L^{1}\left(\mathbb{R}^{d}\right)}=1$. Наконец, берем векторное поле $b$, заданное формулой (1.6.1). Тогда уравнение $L^{*} \mu=0$ с этим коэффициентом $b$ имеет по крайней мере два различных вероятностных решения: одно есть мера $\mu=\varrho d x$, а другое есть мера $\nu=c_{1} v \cdot \mu$, где $c_{1}$ - нормирующая постоянная, а функция $v$ есть решение (не равное константе) уравнения (1.6.2), которое существует по теореме 1.6.4. Представим некоторые примеры таких $a$ и $\varphi$, что $\operatorname{div} a=0$ и выполнены условия теоремы 1.6.4.

ПримеР 1.6.6. Пусть $d=2$. Выберем нечетные функции $q, \psi, \sigma \in C_{b}^{2}\left(\mathbb{R}^{1}\right)$ так, что

$$
q, q \psi, \sigma^{\prime} \in L^{1}\left(\mathbb{R}^{1}\right), \quad \lim _{n \rightarrow \infty} \sigma(n)=1
$$

и $q \psi \geqslant 0$ не есть тождественный нуль. Ясно, что это возможно. Положим

$$
a(x, y):=(0,-q(x)), \quad \varphi(x, y):=\psi(x)+\sigma(y) .
$$

Тогда $\operatorname{div} a=0$. Проверим условия теоремы 1.6 .4 с использованием замечания 1.6.5. Пусть $\Omega_{n}$ - квадрат с вершинами в точках $(n, n),(-n, n),(n,-n)$ и $(-n,-n)$. Тогда

$$
\begin{aligned}
\int_{\partial \Omega_{n}}\left\langle a, \nu_{n}\right\rangle \varphi^{2} d s & =-(\sigma(n)-\sigma(-n)) \int_{-n}^{n} q(x)(2 \psi(x)+\sigma(n)+\sigma(-n)) d x, \\
\int_{\partial \Omega_{n}}\left\langle a, \nu_{n}\right\rangle \varphi d s & =-(\sigma(n)-\sigma(-n)) \int_{-n}^{n} q(x) d x .
\end{aligned}
$$


Следовательно,

$$
\begin{aligned}
& {[\varphi, \varphi]=\lim _{n \rightarrow \infty} \frac{1}{2} \int_{\partial \Omega_{n}}\left\langle a, \nu_{n}\right\rangle \varphi^{2} d s=-2 \int_{-\infty}^{+\infty} q(x) \psi(x) d x<0,} \\
& {[\varphi, 1]=\lim _{n \rightarrow \infty} \int_{\partial \Omega_{n}}\left\langle a, \nu_{n}\right\rangle \varphi d s=-2 \int_{-\infty}^{+\infty} q(x) d x=0 .}
\end{aligned}
$$

Значит, условия теоремы 1.6.4 выполнены. Следовательно, выбирая произвольную строго положительную гладкую вероятностную плотность $\varrho$, можно изготовить снос $b$ (как объяснено в замечании выше) так, что соответствующему уравнению $L^{*} \mu=0$ будут удовлетворять по крайней мере две вероятностные меры, одна из которых - заданная вероятностная мера $\mu=\varrho d x$.

ПРимеР 1.6.7. Пусть $d=2$ и функции $q, \psi, \sigma \in C_{b}^{2}\left(\mathbb{R}^{1}\right)$ не равны нулю тождественно и удовлетворяют следующим условиям:

$$
q, \sigma^{\prime} \in L^{1}\left(\mathbb{R}^{1}\right), \quad q>0, \quad \lim _{n \rightarrow \infty} \sigma(n)=1, \quad \lim _{n \rightarrow \infty} \sigma(-n)=0 .
$$

Положим

$$
a(x, y):=(0,-q(x)), \quad \varphi(x, y):=\psi(x) \sigma(y) .
$$

Опять $\operatorname{div} a=0$. Вычислим $[\varphi, 1]$ и $[\varphi, \varphi]$ с использованием замечания 1.6.5. Пусть $\Omega_{n}-$ квадрат с вершинами в точках $(n, n),(-n, n),(n,-n),(-n,-n)$. Тогда

$$
\begin{aligned}
\int_{\partial \Omega_{n}}\left\langle a, \nu_{n}\right\rangle \varphi^{2} d s & =-\left(\sigma^{2}(n)-\sigma^{2}(-n)\right) \int_{-n}^{n} q(x) \psi^{2}(x) d x \\
\int_{\partial \Omega_{n}}\left\langle a, \nu_{n}\right\rangle \varphi d s & =-(\sigma(n)-\sigma(-n)) \int_{-n}^{n} q(x) \psi(x) d x .
\end{aligned}
$$

Значит,

$$
[\varphi, \varphi]=-\frac{1}{2} \int_{-\infty}^{+\infty} q(x) \psi^{2}(x) d x, \quad[\varphi, 1]=-\int_{-\infty}^{+\infty} q(x) \psi(x) d x .
$$

Чтобы удовлетворялись условия теоремы 1.6.4, достаточно потребовать ортогональность функций $\psi$ и 1 в $L^{2}\left(\mathbb{R}^{1}, q d x\right)$. Замечание 1.6.5 позволяет устроить уравнение $L^{*} \mu=0$ с различными вероятностными решениями, одно из которых - заданная мера $\mu=\varrho d x$.

Легко распространить последний пример на случай $d \geqslant 2$.

ПримеР 1.6.8. Положим $x^{\prime}:=\left(x_{1}, \ldots, x_{d-1}\right)$. Пусть $q, \psi \in C_{b}^{2}\left(\mathbb{R}^{d-1}\right)$ и $\sigma \in$ $C_{b}^{2}\left(\mathbb{R}^{1}\right)$ не равны нулю тождественно и удовлетворяют следующим условиям:

$$
q \in L^{1}\left(\mathbb{R}^{d-1}\right), \quad \sigma^{\prime} \in L^{1}\left(\mathbb{R}^{1}\right), \quad q>0, \quad \lim _{n \rightarrow \infty} \sigma(n)=1, \quad \lim _{n \rightarrow \infty} \sigma(-n)=0 .
$$

Положим

$$
a^{i}(x):=0, \quad \text { если } 1 \leqslant i \leqslant d-1, \quad a^{d}(x):=-q\left(x^{\prime}\right), \quad \varphi(x):=\psi\left(x^{\prime}\right) \sigma\left(x_{d}\right) .
$$


Тогда $\operatorname{div} a=0$ и

$$
[\varphi, \varphi]=-\frac{1}{2} \int_{\mathbb{R}^{d-1}} q\left(x^{\prime}\right) \psi^{2}\left(x^{\prime}\right) d x^{\prime}, \quad[\varphi, 1]=-\int_{\mathbb{R}^{d-1}} q\left(x^{\prime}\right) \psi\left(x^{\prime}\right) d x^{\prime} .
$$

Чтобы удовлетворялись условия теоремы 1.6.4, достаточно потребовать ортогональность функций $\psi$ и 1 в $L^{2}\left(\mathbb{R}^{d-1}, q d x^{\prime}\right)$.

Зафиксируем поле $a \mathrm{c} \operatorname{div} a=0$ и две различные функции $\varphi_{1}$ и $\varphi_{2}$, удовлетворяющие условиям теоремы 1.6.4, и построим два решения $v_{1}$ и $v_{2}$ согласно этой теореме. Эта теорема гарантирует, что $1, v_{1}$ и $1, v_{2}$ - пары линейно независимых функций. При каких условиях на $\varphi_{1}$ и $\varphi_{2}$ три функции $1, v_{1}$ и $v_{2}$ будут линейно независимы? Следующий результат отвечает на этот вопрос и дает некоторую дополнительную информацию.

Теорема 1.6.9. Пусть $n \geqslant 1$. Предположим, что существуют функиии $\varphi_{1}, \varphi_{2}, \ldots, \varphi_{n+1}$ из $C_{b}^{2}\left(\mathbb{R}^{d}\right)$, удовлетворяющие условиям теоремы 1.6.4. Пусть $v_{1}, v_{2}, \ldots, v_{n+1}-$ решения уравнения (1.6.2), порожденные этими функциями согласно теореме 1.6.4. Предположим также, что функиии $1, v_{1}, \ldots, v_{n}$ линейно независимы и для каждого вектора $\alpha=\left(\alpha_{1}, \ldots, \alpha_{n}\right) \in \mathbb{R}^{n}$ выполнено следующее неравенство:

$$
\left[\varphi_{n+1}-\sum_{k=1}^{n} \alpha_{k} \varphi_{k}, \varphi_{n+1}-\sum_{k=1}^{n} \alpha_{k} \varphi_{k}\right]<0 .
$$

Тогда функции $1, v_{1}, \ldots, v_{n}, v_{n+1}$ линейно независимы.

ЗАмЕчАНИЕ 1.6.10. Пусть $\Phi=\left(\Phi_{i j}\right)_{i, j \leqslant n}$ и $h=\left(h_{i}\right)_{i \leqslant n}$, где

$$
\Phi_{i j}=\frac{\left[\varphi_{i}, \varphi_{j}\right]+\left[\varphi_{j}, \varphi_{i}\right]}{2}, \quad h_{i}=\left[\varphi_{i}, \varphi_{n+1}\right]+\left[\varphi_{n+1}, \varphi_{i}\right], \quad 1 \leqslant i, j \leqslant n .
$$

Положим $h_{0}:=\left[\varphi_{n+1}, \varphi_{n+1}\right]$. Тогда неравенство (1.6.4) может быть переписано в следующем виде:

$$
\langle\Phi \alpha, \alpha\rangle-\langle h, \alpha\rangle+h_{0}<0 .
$$

Следовательно, чтобы обеспечить условие (1.6.4), достаточно добиться следующего: матрица $\Phi$ строго отрицательна и

$$
4 h_{0}<\left\langle\Phi^{-1} h, h\right\rangle .
$$

В частности, если $n=1$, то неравенство (1.6.5) имеет следующий очень простой вид:

$$
\left(\left[\varphi_{1}, \varphi_{2}\right]+\left[\varphi_{2}, \varphi_{1}\right]\right)^{2}<4\left[\varphi_{1}, \varphi_{1}\right]\left[\varphi_{2}, \varphi_{2}\right]
$$

ЗАмЕЧАНИЕ 1.6.11. Чтобы проверить условие (1.6.4), полезно иметь в виду следующее выражение для $\left[\varphi_{i}, \varphi_{j}\right]+\left[\varphi_{j}, \varphi_{i}\right]$. Пусть $\Omega_{n}-$ возрастающие области с кусочно-гладкими границами и $\mathbb{R}^{d}=\bigcup_{n=1}^{\infty} \Omega_{n}$. Поскольку $\operatorname{div} a=0$, то мы имеем

$$
\begin{aligned}
{\left[\varphi_{i}, \varphi_{j}\right]+\left[\varphi_{j}, \varphi_{i}\right] } & =\int_{\mathbb{R}^{d}}\left\langle a, \nabla \varphi_{i}\right\rangle \varphi_{j} d x+\left[\varphi_{j}, \varphi_{i}\right] \\
& =\lim _{n \rightarrow \infty} \int_{\Omega_{n}}\left\langle a, \nabla \varphi_{i}\right\rangle \varphi_{j} d x+\left[\varphi_{j}, \varphi_{i}\right]=\lim _{n \rightarrow \infty} \int_{\partial \Omega_{n}}\left\langle a, \nu_{n}\right\rangle \varphi_{i} \varphi_{j} d s
\end{aligned}
$$

где $\nu_{n}$ - внешняя нормаль на $\partial \Omega_{n}$. 
ЗАМЕЧАНИЕ 1.6.12. Предположим, что для заданного гладкого векторного поля $a$ c $\operatorname{div} a=0$ существуют такие функции $\varphi_{1}, \ldots, \varphi_{n+1}$, что выполнены условия теоремы 1.6.9. Предположим, что дана строго положительная бесконечно дифференцируемая функция $\varrho$ с $\|\varrho\|_{L^{1}\left(\mathbb{R}^{d}\right)}=1$. Тогда уравнение $L^{*} \mu=0$ с коэффициентом $b$, выраженным через $a$ и $\varrho$ по формуле (1.6.1), имеет по крайней мере $n+1$ линейно независимое вероятностное решение, одно из которых есть мера $\mu=\varrho d x$, а $n$ других - меры $\nu_{i}=c_{i} v_{i} \cdot \mu$, где $c_{i}-$ нормирующие постоянные, а функции $v_{i}$ являются непостоянными решениями уравнения (1.6.2), порожденными функциями $\varphi_{i}$.

Представим явный пример $a, \varphi_{1}$ и $\varphi_{2}$, в котором выполнены условия теоремы 1.6.9.

ПРИмеР 1.6.13. Пусть $x^{\prime}:=\left(x_{1}, \ldots, x_{d-1}\right)$ и функции $q, \psi_{1}, \psi_{2} \in C_{b}^{2}\left(\mathbb{R}^{d-1}\right)$ и $\sigma \in C_{b}^{2}\left(\mathbb{R}^{1}\right)$ не равны нулю тождественно. Предположим, что

$$
q \in L^{1}\left(\mathbb{R}^{d-1}\right), \quad q>0, \quad \sigma^{\prime} \in L^{1}\left(\mathbb{R}^{1}\right), \quad \lim _{n \rightarrow \infty} \sigma(n)=1, \quad \lim _{n \rightarrow \infty} \sigma(-n)=0 .
$$

Положим

$$
\begin{array}{ll}
a^{k}(x):=0, \quad \text { если } 1 \leqslant k \leqslant d-1, & a^{d}(x):=-q\left(x^{\prime}\right), \\
\varphi_{1}(x):=\psi_{1}\left(x^{\prime}\right) \sigma\left(x_{d}\right), & \varphi_{2}(x):=\psi_{2}\left(x^{\prime}\right) \sigma\left(x_{d}\right) .
\end{array}
$$

Тогда $\operatorname{div} a=0$ и при $1 \leqslant i, j \leqslant 2$ имеем

$$
\begin{aligned}
{\left[\varphi_{i}, \varphi_{j}\right]+\left[\varphi_{j}, \varphi_{i}\right] } & =-\int_{\mathbb{R}^{d-1}} \psi_{i}\left(x^{\prime}\right) \psi_{j}\left(x^{\prime}\right) q\left(x^{\prime}\right) d x^{\prime} \\
{\left[\varphi_{i}, 1\right] } & =-\int_{\mathbb{R}^{d-1}} \psi_{i}\left(x^{\prime}\right) q\left(x^{\prime}\right) d x^{\prime} .
\end{aligned}
$$

Чтобы выполнялись условия теоремы 1.6 .9 , достаточно потребовать ортогональность функций $1, \psi_{1}, \psi_{2}$ в $L^{2}\left(\mathbb{R}^{d-1}, q d x^{\prime}\right)$.

Этот пример легко распространить на случай произвольного числа функций $\varphi_{i}$. Более того, можно дать пример уравнения $L^{*} \mu=0$, которое имеет счетную последовательность линейно независимых вероятностных решений. В частности, пространство решений такого уравнения в классе ограниченных мер бесконечномерно. Достаточно найти последовательность положительных ограниченных решений $\left\{v_{i}\right\}_{i \geqslant 1}$ уравнения (1.6.2), для которых функции 1 , $\left\{v_{i}\right\}_{i \geqslant 1}$ линейно независимы. Согласно теореме 1.6 .9 и замечанию 1.6 .10 , достаточно выбрать векторное поле $a$ c $\operatorname{div} a=0$ и последовательность функций $\left\{\varphi_{i}\right\}_{i \in \mathbb{N}}$, удовлетворяющих условиям теоремы 1.6.4, таким образом, что для каждого $n$ функции $\varphi_{1}, \ldots, \varphi_{n+1}$ удовлетворяют условию (1.6.5).

ПримеР 1.6.14. Положим $x^{\prime}:=\left(x_{1}, \ldots, x_{d-1}\right)$. Пусть $q, \psi_{i} \in C_{b}^{2}\left(\mathbb{R}^{d-1}\right)$, где $i \in \mathbb{N}$, и пусть $\sigma \in C_{b}^{2}\left(\mathbb{R}^{1}\right)$. Предположим, что

$$
q \in L^{1}\left(\mathbb{R}^{d-1}\right), \quad q>0, \quad \sigma^{\prime} \in L^{1}\left(\mathbb{R}^{1}\right), \quad \lim _{n \rightarrow \infty} \sigma(n)=1, \quad \lim _{n \rightarrow \infty} \sigma(-n)=0 .
$$

Положим

$$
\begin{array}{rlrl}
a^{k}(x):=0 & \text { при } 1 \leqslant k \leqslant d-1, & & a^{d}(x):=-q\left(x^{\prime}\right), \\
\varphi_{i}(x):=\psi_{i}\left(x^{\prime}\right) \sigma\left(x_{d}\right), & i \in \mathbb{N} .
\end{array}
$$


Тогда для всех $i, j \geqslant 1$ имеем

$$
\begin{aligned}
{\left[\varphi_{i}, \varphi_{j}\right]+\left[\varphi_{j}, \varphi_{i}\right] } & =-\int_{\mathbb{R}^{d-1}} \psi_{i}\left(x^{\prime}\right) \psi_{j}\left(x^{\prime}\right) q\left(x^{\prime}\right) d x^{\prime} \\
{\left[\varphi_{i}, 1\right] } & =-\int_{\mathbb{R}^{d-1}} \psi_{i}\left(x^{\prime}\right) q\left(x^{\prime}\right) d x^{\prime}
\end{aligned}
$$

Пусть $1,\left\{\psi_{i}\right\}_{i \in \mathbb{N}}$ - ортонормированная система в $L^{2}\left(\mathbb{R}^{d-1}, q d x^{\prime}\right)$. Тогда для каждого $n$ выполнено условие (1.6.5) для функций $1, \varphi_{1}, \ldots, \varphi_{n+1}$, поскольку матрица $\Phi$ диагональна и $\Phi_{i i}=-1 / 2$, значит, она отрицательна, $h=0, h_{0}=$ $\left[\psi_{n+1}, \psi_{n+1}\right]<0$.

ПримеР 1.6.15. Мы уже упоминали пример из [11], [12], в котором уравнение $L^{*} \mu=0$ имеет по крайней мере два различных вероятностных решения. Теперь мы можем показать, что на самом деле оно имеет счетную последовательность линейно независимых решений, являющихся вероятностными мерами. Мы имеем

$$
b^{k}(x)=-x_{k}-2 x_{\sigma(k)} \exp \left(\frac{x_{k}^{2}-x_{\sigma(k)}^{2}}{2}\right), \quad \varrho(x)=(2 \pi)^{-d / 2} \exp \left(-\frac{|x|^{2}}{2}\right),
$$

где $\sigma:\{1,2,3, \ldots, d\} \rightarrow\{1,2,3, \ldots, d\}$ взаимно однозначно и $\sigma(k) \neq k$. Тогда

$$
a^{k}(x)=-2(2 \pi)^{d / 2} x_{\sigma(k)} \exp \left(-x_{\sigma(k)}^{2}-\frac{1}{2} \sum_{i \neq k} x_{i}^{2}\right) .
$$

Пусть $\mathbb{R}_{+}^{d-1}:=\mathbb{R}^{d-1} \cap\left\{x_{\sigma(d)}>0\right\}$ и $x^{\prime}:=\left(x_{1}, \ldots, x_{d-1}\right)$. Выберем функции $\omega, \psi_{i}^{* *} \in C_{0}^{\infty}\left(\mathbb{R}_{+}^{d-1}\right)$, где $i \in \mathbb{N}$, с дизъюнктными носителями так, что

$$
-\int_{\mathbb{R}_{+}^{d-1}} \omega\left(x^{\prime}\right) a^{d}\left(x^{\prime}\right) d x^{\prime}=1 .
$$

Положим

$$
\psi_{i}^{*}\left(x^{\prime}\right):=\psi_{i}^{* *}\left(x^{\prime}\right)+\omega\left(x^{\prime}\right) \int_{\mathbb{R}_{+}^{d-1}} \psi_{i}^{* *}\left(y^{\prime}\right) a^{d}\left(y^{\prime}\right) d y^{\prime}, \quad x^{\prime} \in \mathbb{R}_{+}^{d-1} .
$$

Заметим, что $\operatorname{div} a=0$ и

$$
\int_{\mathbb{R}_{+}^{d-1}} \psi_{i}^{*}\left(x^{\prime}\right) a^{d}\left(x^{\prime}\right) d x^{\prime}=0 \quad \text { для каждого } i \geqslant 1 .
$$

Легко видеть, что функции $\psi_{i}^{*}$ линейно независимы. Применим процесс ортогонализации в пространстве $L^{2}\left(\mathbb{R}_{+}^{d-1},-a^{d}\left(x^{\prime}\right) d x^{\prime}\right)$ к системе $\left\{\psi_{i}^{*}\right\}_{i \geqslant 1}$ и получим функции $\left\{\psi_{i}\right\}_{i \geqslant 1}$. Заметим, что полученные функции обладают следующими свойствами: $\lim _{\left|x^{\prime}\right| \rightarrow \infty} \psi_{i}\left(x^{\prime}\right)=0$ и

$$
\int_{\mathbb{R}_{+}^{d-1}} \psi_{i}\left(x^{\prime}\right) a^{d}\left(x^{\prime}\right) d x^{\prime}=0, \quad \int_{\mathbb{R}_{+}^{d-1}} \psi_{i}\left(x^{\prime}\right) \psi_{j}\left(x^{\prime}\right) a^{d}\left(x^{\prime}\right) d x^{\prime}=0
$$

для всех $i, j \geqslant 1$. Продолжим $\psi_{i}$ на все пространство $\mathbb{R}^{d-1}$ нулем вне $\mathbb{R}_{+}^{d-1}$. Ясно, что получатся функции из $C_{0}^{\infty}\left(\mathbb{R}^{d-1}\right)$, поскольку $\omega$ и $\psi^{* *}$ имеют ком- 
пактные носители в полупространстве $\mathbb{R}_{+}^{d-1}$. Пусть $\sigma$ - такая гладкая функция, что $\sigma^{\prime} \in L^{1}\left(\mathbb{R}^{1}\right), \lim _{n \rightarrow \infty} \sigma(n)=1$ и $\lim _{n \rightarrow \infty} \sigma(-n)=0$. Положим $\varphi_{i}(x):=$ $\psi_{i}\left(x^{\prime}\right) \sigma\left(x_{d}\right)$. Согласно теореме 1.6.4, можно построить непостоянные решения, соответствующие нашим функциям $\varphi_{i}$. Аналогично предыдущему примеру мы получим последовательность решений $1, v_{1}, v_{2}, \ldots$, которые линейно независимы.

Наконец, приведем еще одно достаточное условие существования непостоянного положительного ограниченного решения уравнения (1.6.2).

ПреДЛОЖеНИЕ 1.6.16. Предположим, что существует такая функиия $\varphi \in C_{b}^{2}\left(\mathbb{R}^{d}\right)$, чmo

$$
2 \sup _{x \in \mathbb{R}^{d}}|\varphi(x)| \int_{\mathbb{R}^{d}}\left|L_{\mu} \varphi\right| d x<\int_{\mathbb{R}^{d}}|\nabla \varphi|^{2} \varrho d x .
$$

Тогда существует ограниченное положительное решение уравнения (1.6.2), не являющееся постоянной.

Следующий пример демонстрирует применение этого предложения.

ПримеР 1.6.17. Пусть $d=2$ и $\varrho_{1}, \varrho_{2} \in C^{\infty}\left(\mathbb{R}^{1}\right) \cap L^{1}\left(\mathbb{R}^{1}\right)$ - такие положительные функции, что $\left\|\varrho_{1}\right\|_{L^{1}}=\left\|\varrho_{2}\right\|_{L^{1}}=1$. Положим $\varrho(x, y):=\varrho_{1}(x) \varrho_{2}(y)$,

$$
\begin{gathered}
\varphi(x, y):=\int_{-\infty}^{x} \varrho_{1}(s) d s+\int_{-\infty}^{y} \varrho_{2}(s) d s \\
a^{1}(x, y):=-2 \varrho_{2}^{\prime}(y) \varrho_{2}(y)+c(y) \varrho_{2}(y), \quad a^{2}(x, y):=-2 \varrho_{1}^{\prime}(x) \varrho_{2}(x)+d(x) \varrho_{1}(x) .
\end{gathered}
$$

Тогда $\operatorname{div} a=0$ и

$$
L_{\mu} \varphi(x, y)=(d(x)+c(y)) \varrho(x, y) .
$$

Чтобы выполнялись условия предложения, достаточно иметь следующую оценку:

$$
4 \int_{\mathbb{R}^{2}}|d(x)+c(y)| \varrho(x, y) d x d y<\int_{\mathbb{R}^{2}}\left[\varrho_{1}(x)^{2}+\varrho_{2}(y)^{2}\right] \varrho(x, y) d x d y .
$$

ЗАМЕчАНИЕ 1.6.18. При условиях предложения 1.6.16 мы имеем

$$
\int_{\mathbb{R}^{d}} \operatorname{div}(\varrho \nabla \varphi) d x=0 .
$$

Значит, оценка в условии этого предложения может быть заменена следующей:

$$
L_{\mu} \varphi \geqslant 0 \quad \text { и } \quad \int_{\mathbb{R}^{d}}|\nabla \varphi|^{2} \varrho d x+2 \sup _{x \in \mathbb{R}^{d}}|\varphi(x)| \int_{\mathbb{R}^{d}}(a, \nabla \varphi) d x>0 .
$$

Следует отметить, что остается неизвестным, может ли уравнение $L^{*} \mu=0$ иметь лишь конечное число (но более одного) линейно независимых вероятностных решений в случае гладкого $b$. Для сингулярного $b$ такие примеры легко построить на прямой. 
1.7. Проблемы единственности. Здесь мы приведем достаточные условия на $A$ и $b$, при которых $\mathscr{M}_{\mathrm{ell}}^{A, b}$ содержит самое большее один элемент (множества $\mathscr{M}_{\mathrm{ell}}^{A, b}$ и $\mathscr{M}_{\mathrm{ell}, \mathrm{md}}^{A, b}$ определены в (1.1.2) и (1.5.9) соответственно). Для получения наилучших возможных результатов необходимо отделить вопрос единственности от вопроса существования. Выше мы видели, что в случае $d=1$ для всякой локально интегрируемой по Лебегу функции $b$ уравнение $L_{\mathrm{I}, b}^{*}=0$ имеет самое большее одно решение в классе вероятностных мер. Сначала приведем один результат из [7].

ТеОрема 1.7.1. Пусть $|b| \in L_{\mathrm{loc}}^{p}\left(\mathbb{R}^{d}\right)$ для некоторого $p>d \geqslant 2$. Тогда существует не более одной мерь $\mu \in \mathscr{M}_{\mathrm{ell}}^{\mathrm{I}, b}$, плотность $\varrho$ которой имеет то свойство, что

$$
|b \varrho-\nabla \varrho| \in L^{1}\left(\mathbb{R}^{d}\right) .
$$

Неизвестно примеров неединственности в случае, когда $A=\mathrm{I}, b$ гладко и $|b| \in L^{1}(\mu)$.

Имеются также результаты, не использующие каких-либо предположений о логарифмическом градиенте решения.

ТЕОРема 1.7.2. Пусть выполнены условия (А1) и (А2) из (1.5.2) и $\Omega$ связно. Тогда

$$
\mathscr{M}_{\mathrm{ell}, \mathrm{md}}^{A, b} \neq \varnothing \quad \Rightarrow \quad \# \mathscr{M}_{\mathrm{ell}}^{A, b}=1 .
$$

Отметим, что обратное утверждение неверно во всяком случае при $d=1$ (см. замечание 1.7.19 ниже).

Важность этого результата видна из того факта, что единственность решения обеспечивается информацией о некотором решении.

СЛЕДСТВИЕ 1.7.3. Если при предположениях теоремы 1.7 .2 существует мера $\mu \in \mathscr{M}_{\mathrm{ell}}^{A, b}$, инвариантная относительно соответствующей полугруппы $\left(T_{t}^{\mu}\right)_{t \geqslant 0}, m o \# \mathscr{M}_{\mathrm{ell}}^{A, b}=1$.

ЗАмЕчАниЕ 1.7.4. Предположим, что $\Omega$ связно. Тогда при предположениях теоремы 1.7.2 выполнено неравенство $\# \mathscr{M}_{\mathrm{ell}}^{A, b} \leqslant 1$, если и только если для всяких мер $\mu, \nu \in \mathscr{M}_{\mathrm{ell}}^{A, b}$ мы имеем $|\mu-\nu| \in \mathscr{M}_{\mathrm{ell}}^{A, b}$. В самом деле, указанное условие дает $\mu=\nu$, поскольку иначе мера $|\mu-\nu|$ имеет строго положительную непрерывную плотность. Обратное тривиально.

Это приводит к следующему вопросу: если знакопеременная мера $\mu$ удовлетворяет уравнению $L_{A, b}^{*} \mu=0$, то удовлетворяет ли $|\mu|$ тому же самому уравнению? В общем случае это неверно, даже если $A=\mathrm{I}$ и $b$ гладко. В самом деле, как объяснено выше, в случае, когда всякая вероятностная мера, удовлетворяющая уравнению $L_{A, b}^{*} \mu=0$, обладает положительной непрерывной плотностью, что имеет место при $a^{i j} \in W_{\mathrm{loc}}^{p, 1}\left(\mathbb{R}^{d}\right),|b| \in L_{\mathrm{loc}}^{p}\left(\mathbb{R}^{d}\right)$ с $p>d$ и невырожденном $A$, неединственность решений этого уравнения в $\mathscr{P}\left(\mathbb{R}^{d}\right)$ всегда дает знакопеременные решения, абсолютные значения которых не являются решениями. Конечно, это не удивительно для локально интегрируемых решений, например, модуль гармонической функции может не быть гармонической функцией, но для глобально интегрируемых решений это явление более интересно. Здесь уместно отметить, что если $\mu$ - инвариантная мера полугруппы $\left(T_{t}\right)_{t \geqslant 0}$, генератор которой продолжает $\left(L_{A, b}, C_{0}^{\infty}\right)$, то $|\mu|$ - также инвариантная 
мера, что еще раз выявляет некоторую разницу между инвариантными мерами полугрупп и инфинитезимально инвариантными мерами. Как мы увидим ниже, при наших типичных предположениях относительно $A$ и $b$, привлекающих функции Ляпунова, уравнение $L_{A, b}^{*} \mu=0$ имеет одно решение в $\mathscr{P}\left(\mathbb{R}^{d}\right)$. Однако мы не знаем, является ли в этом случае пространство всех решений в классе ограниченных знакопеременных мер одномерным.

Из предыдущего пункта нам известно, что для $\Omega=\mathbb{R}^{d}$ действительно может случиться так, что \# $\mathscr{M}_{\mathrm{ell}}^{A, b}>1$. Результаты выше дают условия на $A$ и $b$, при которых $\# \mathscr{M}_{\mathrm{ell}}^{A, b}=1$. Другие результаты будут приведены ниже. Однако возникает следующий вопрос, уже затронутый в замечании 1.5.18: может ли полугруппа $\left(T_{t}^{\mu}\right)_{t \geqslant 0}$ иметь инвариантные меры, если $\mu$ не инвариантна, или может ли она иметь инвариантные меры, отличные от $\mu$, в случае, когда $\mu$ инвариантна?

Следующий результат из [9] отвечает на этот вопрос в случае $p>d+2$ (этот результат дополняет более ранний результат из [7] и при исключительно локальных предположениях дает утвердительный ответ на вопрос, поставленный С. Р. С. Вараданом в [87]). Видимо, это же верно при нашем стандартном предположении $p>d$.

ТеОРема 1.7.5. Предположим, что $\mu \in \mathscr{M}_{\mathrm{ell}}^{A, b} u$ выполнены условия (A1) u (A2) из (1.5.2). Кроме того, пусть $p>d+2$. Тогда на $\Omega$ существуют такие субвероятностные ядра $K_{t}(\cdot, d y), t>0$, что

$$
K_{t}(x, d y)=p_{A, b}(t, x, y) d y,
$$

где $p_{A, b}(t, x, y)$ - локально гёльдеровы неотрицательные функиии, определенные на множестве $(0,+\infty) \times \Omega \times \Omega$, и для каждого $f \in L^{1}(\Omega, \mu)$ функция

$$
x \mapsto K_{t} f(x):=\int_{\Omega} f(y) p_{A, b}(t, x, y) d y
$$

есть такая $\mu$-версия $T_{t}^{\mu} f$, что функиия $(t, x) \mapsto K_{t} f(x)$ непрерьвна на множестве $(0,+\infty) \times \Omega$. Функиия $p_{A, b}$ положительна, если $\Omega$ связно.

Кроме того, если $\Omega$ связно и $\nu$-ограниченная борелевская мера на $\Omega$, инвариантная для $\left(K_{t}\right)_{t} \geqslant 0$, m.e.

$$
\nu=K_{t}^{*} \nu(d y):=\int_{\Omega} K_{t}(x, d y) \nu(d x) \quad \forall t \geqslant 0,
$$

то $\nu=c \mu$ для некоторой постоянной с. $B$ частности, если $\nu \neq 0$, то $\mu$ также инвариантна. Значит, $\left(K_{t}\right)_{t \geqslant 0}$ не может иметь инвариантных вероятностных мер, отличных от $\mu$.

ЗАмЕчАниЕ 1.7.6. По этой теореме полугруппа $\left(T_{t}^{\mu}\right)_{t \geqslant 0}$ является сильно феллеровской: она переводит ограниченные борелевские функции в непрерывные функции. Кроме того, она стохастически непрерывна в том смысле, что $\lim _{t \rightarrow 0} T_{t}^{\mu} I_{B(z, r)}(x)=1$ для всех $z \in \Omega$ и $r>0$ таких, что $B(z, r) \subset \Omega$. Это вытекает из последнего утверждения в теореме 1.5 .7 и оценки $T_{t}^{\mu} I_{B(z, r)} \geqslant T_{t}^{\mu} \psi$, где функция $\psi \in C_{0}^{\infty}(\Omega)$ такова, что $0 \leqslant \psi \leqslant 1, \psi(z)=1$ и $\psi=0$ вне $B(z, r)$. Если $\Omega$ связно, то имеем эквивалентность всех мер

$$
B \mapsto T_{t}^{\mu} I_{B}(x)=K_{t}^{*} \delta_{x}(B)=\int_{B} p_{A, b}(t, x, y) d y .
$$


Следовательно, если $\mu$ инвариантна для $\left(T_{t}^{\mu}\right)_{t \geqslant 0}$ (что не автоматически выполняется в нашей ситуации!), то, согласно теореме Дуба (см. [88] или [89; §4.2]), для каждого борелевского множества $B \subset \Omega$ мы получим

$$
\lim _{t \rightarrow \infty} T_{t}^{\mu} I_{B}(x)=\mu(B) \quad \forall x \in \Omega,
$$

где рассматривается совместно непрерывная версия $T_{t}^{\mu} I_{B}(x)$. Конечно, из этого следует, что $\lim _{t \rightarrow \infty}\left\|T_{t}^{\mu} f-f\right\|_{L^{p}(\mu)}=0$ для всех $f \in L^{p}(\mu)$.

ЗАмЕчание 1.7.7. Таким образом, один из основных результатов настоящего пункта - следующая альтернатива: при локальных условиях (A1) и (A2) из (1.5.2) в случае, когда уравнение $L_{A, b}^{*} \mu=0$ имеет решение $\mu$ в классе вероятностных мер, либо $\mu$ является единственной вероятностной мерой, удовлетворяющей этому уравнению, ее ассоциированная полугруппа $\left(T_{t}^{\mu}\right)_{t \geqslant 0}$ также единственна, причем если $\mu$ инвариантна для $\left(T_{t}^{\mu}\right)_{t \geqslant 0}$ (что не следует автоматически из единственности $\mu$ даже на прямой, см. замечание 1.7.19), то других инвариантных вероятностных мер для $\left(T_{t}^{\mu}\right)_{t \geqslant 0}$ нет, либо никакая вероятностная мера $\mu$, удовлетворяющая этому уравнению, не инвариантна для ее полугруппы $\left(T_{t}^{\mu}\right)_{t \geqslant 0}$, причем тогда для каждой такой меры $\mu$ обязательно имеются разные ассоциированные полугруппы в $L^{1}(\mu)$. Важный качественный вывод состоит в том, что при разумных локальных предположениях на $A$ и $b$ имеет место единственность инвариантных вероятностных мер для нашей специальной ассоциированной полугруппы, но нет единственности для эллиптического уравнения, причем лучшая гладкость коэффициентов не помогает получить единственность решений эллиптического уравнения.

Сделаем простое наблюдение о крайних точках выпуклого множества $\mathscr{M}_{\mathrm{ell}}^{A, b}$. Определим ext $\mathscr{M}_{\mathrm{ell}}^{A, b}$ как множество всех мер $\mu \in \mathscr{M}_{\mathrm{ell}}^{A, b}$, которые не могут быть записаны как нетривиальные выпуклые комбинации двух других элементов $\mathscr{M}_{\text {ell }}^{A, b}$.

ПреДЛОЖЕНИЕ 1.7.8. Пусть $\mu \in \mathscr{M}_{\mathrm{ell}}^{A, b}$. Тогда следующие утверждения равносильны:

(i) $\mu \in \operatorname{ext} \mathscr{M}_{\text {ell }}^{A, b}$;

(ii) если $\varrho \in L^{\infty}(\Omega, \mu) u \varrho \cdot \mu \in \mathscr{M}_{\mathrm{ell}}^{A, b}$, mo $\varrho=1 \mu$-n.в.

СЛЕДСТВИЕ 1.7.9. Предположим, что выполненъ условия (A1) и (А2) из (1.5.2). Пусть $\mu \in \operatorname{ext} \mathscr{M}_{\mathrm{ell}}^{A, b}$. Тогда для всех $\nu \in \mathscr{M}_{\mathrm{ell}}^{A, b} \backslash\{\mu\}$ функиия $d \nu / d \mu$ неограничена.

Конечно, может случиться, что $\mathscr{M}_{\mathrm{ell}}^{A, b}=\varnothing$ (например, если $\Omega=\mathbb{R}^{d}, A=\mathrm{I}$, $b \equiv 0)$. Однако даже в случае, когда $\Omega=\mathbb{R}^{d}, A=\mathrm{I}$ и $b$ бесконечно дифференцируемо, может также случиться, что $\# \mathscr{M}_{\mathrm{ell}}^{A, b}>1$ и, следовательно, $\mathscr{M}_{\mathrm{ell}, \mathrm{md}}^{A, b}$ пусто (см. пример 1.6.3).

Рассмотрим ряд примеров, заимствованных из [12].

ПримеР 1.7.10. Пусть выполнены условия (A1) и (А2) из (1.5.2) и $\Omega$ связно. Предположим, что существуют такие неограниченная компактная функция $V \in C^{2}(\Omega)$, число $\alpha>0$ и компактное множество $K$, что

$$
L_{A, b} V(x) \leqslant \alpha V(x) \quad \text { для почти всех } x \in \Omega \backslash K .
$$

Тогда $\# \mathscr{M}_{\mathrm{ell}}^{A, b} \leqslant 1$. Аналогичный результат верен для оператора $\mathscr{L}_{A, b}$. 
Например, беря функцию $V(x)=\ln \left(|x|^{2}+1\right)$ на $\mathbb{R}^{d}$ и замечая, что

$$
L_{A, b} V(x)=\frac{2 \operatorname{trace} A(x)}{|x|^{2}+1}-\frac{4\langle A(x) x, x\rangle}{\left(|x|^{2}+1\right)^{2}}+\frac{2\langle b(x), x\rangle}{|x|^{2}+1},
$$

приходим к следующему результату.

ПРИмеР 1.7.11. Пусть выполнены условия (A1) и (А2) из (1.5.2). Предположим, что $\Omega=\mathbb{R}^{d}$ и существует такое число $C>0$, что п. в. вне некоторого шара мы имеем

$$
-\frac{2}{1+|x|^{2}}\langle A(x) x, x\rangle+\operatorname{trace} A(x)+\langle b(x), x\rangle \leqslant C|x|^{2} \ln |x| .
$$

Тогда $\# \mathscr{M}_{\mathrm{ell}}^{A, b} \leqslant 1$. В частности, если $A$ равномерно ограничено, то достаточно иметь оценку $\langle b(x), x\rangle \leqslant C|x|^{2} \ln |x|$ п. в. вне некоторого шара. Например, достаточна оценка $|b(x)| \leqslant c+c|x| \ln |x|$. Однако оценки $|b(x)| \leqslant c+c|x|(\ln (2+|x|))^{r}$ при $r>1$ уже не хватит: в примере 1.6.3 возьмем гладкую функцию $f$ с $f^{\prime}(s)=$ $c|s|^{-1}(\ln s)^{-r}$ вне $[-2,2]$. Здесь годится любая положительная интегрируемая функция $f^{\prime}$, что даст пример неединственности, в котором $|b(x)| \leqslant C / f^{\prime}(|x|)$.

ЗАмечание 1.7.12. (i) Рассуждение, аналогичное [50; замечание 1.11 (ii)], показывает, что если имеются ограниченная функция $u \in C^{2}\left(\mathbb{R}^{d}\right)$ и число $\alpha>0$ такие, что $u>0$ и $L_{A, b} u \geqslant \alpha u$, то $\mu$ не инвариантна для $\left(T_{t}^{\mu}\right)_{t \geqslant 0}$.

(ii) Пусть $A$ удовлетворяет условию (A1) из (1.5.2) и $b^{i} \in W_{\text {loc }}^{p, 1}(\Omega)$. Предположим, что существует неограниченная компактная функция $V \in C^{3}(\Omega)$. Пусть $\theta(x)=\left|L_{A, b} V(x)\right|+1$. Тогда оператор $L_{A / \theta, b / \theta}=\theta^{-1} L_{A, b}$ удовлетворяет условиям (A1) и (А2) из (1.5.2) и условию (1.7.1), поскольку $\theta^{-1} L_{A, b} V \leqslant 1$. Это показывает, что условие (1.7.1) всегда можно получить подходящим масштабированием данного оператора (с разумными коэффициентами). Кроме того, мы видим, что существует самое большее одна вероятностная плотность $f \in L^{1}(\Omega, \mu)$, для которой $f \cdot \mu \in \mathscr{M}_{\mathrm{ell}}^{A, b}$ и $\theta f \in L^{1}(\Omega, \mu)$. В самом деле, $\left(\theta^{-1} L_{A, b}\right)^{*}(\theta f \cdot \mu)=0$.

В следующем примере для вывода о том, что $\mu$ является единственным элементом в $\mathscr{M}_{\mathrm{ell}}^{A, b}$, привлекается некоторая информация о самой мере $\mu$. Этот результат дает более слабые достаточные условия, чем [50; предложение 1.10 (a)] (где доказательство требует некоторых исправлений).

ПРимеР 1.7.13. Пусть выполнены условия (А1) и (А2) из (1.5.2). Предположим, что $\Omega=\mathbb{R}^{d}, \mu \in \mathscr{M}_{\mathrm{ell}}^{A, b}$ и

$$
\lim _{j \rightarrow \infty} \int_{j \leqslant|x| \leqslant j+1}\left[\left|a^{i k}\right|+\left|b-\beta_{\mu, A}\right|\right] d \mu=0
$$

для всех $i, k$. Тогда $\# \mathscr{M}_{\mathrm{ell}}^{A, b}=1$. В частности, достаточно иметь интегрируемость $a^{i k}$ и $\left|b-\beta_{\mu, A}\right|$ относительно $\mu$.

В случае оператора $\mathscr{L}_{A, b}$ на связном полном римановом многообразии $M$ достаточным условием того, что $\mu$ является единственной мерой в $\mathscr{M}_{\mathrm{ell}}^{A, b}$, является соотношение

$$
\lim _{j \rightarrow \infty} \int_{B_{j+1} \backslash B_{j}}\left[\|A\|+\left|b-A \beta_{\mu}\right|\right] d \mu=0 .
$$

где $B_{j}$ - геодезический шар радиуса $j$ с центром в фиксированной точке $o \in M$. $\mathrm{B}$ частности, достаточно иметь интегрируемость $\|A\|$ и $\left|b-A \beta_{\mu}\right|$ относительно $\mu$. 
Наконец, выделим специальный случай примера выше, в котором, однако, не требуется знать $\beta_{\mu}$.

Пример 1.7.14. Пусть $\Omega=\mathbb{R}^{d}$ и $\mu \in \mathscr{M}_{\mathrm{ell}}^{A, b}$. Предположим, что $A$ и $A^{-1}$ равномерно ограничены, $A$ глобально липшицево, $|b| \in L_{\mathrm{loc}}^{p}(\Omega)$, где $p>d$. Пусть $|b| \in L^{2}\left(\mathbb{R}^{d}, \mu\right)$. Тогда \# $\mathscr{M}_{\mathrm{ell}}^{A, b}=1$.

ЗАмЕЧАНИЕ 1.7.15. В последнем примере нельзя опустить предположение, что $|b| \in L_{\mathrm{loc}}^{p}(\Omega, d x), p>d$. Это ясно из примера 1.6.1. Если в указанном примере взять $\mu=\varrho d x$ с $\varrho(x)=C x^{2} \exp \left(-x^{2}\right)$, где $C$ - нормирующая постоянная, то $\mu$ удовлетворяет уравнению $L_{1, b}^{*} \mu=0$ с $b(x)=2 x^{-1}-2 x$. Вероятностная мера с плотностью $g$ такой, что $g(x)=f(x) / 2$ при $x<0$ и $g(x)=3 f(x) / 2$ при $x \geqslant 0$, есть другое решение. Для обоих решений оператор $L_{1, b}$ даже симметричен, а коэффициент $b$ квадратично интегрируем относительно обеих мер. Можно проверить, что эти две меры не только являются решениями одного и того же эллиптического уравнения, но также и инвариантны для одной и той же марковской полугруппы, ассоциированной с оператором $L_{1, b}$ (в рассматриваемом случае такая полугруппа единственна).

Изложенные выше результаты имеют следующий характер: либо утверждается, что $\# \mathscr{M}_{\mathrm{ell}}^{A, b} \leqslant 1$, либо говорится, что $\# \mathscr{M}_{\mathrm{ell}}^{A, b}=1$, но существование некоторого решения входит в предположения. Однако теоремы существования, данные в п. 1.4 в терминах функций Ляпунова, гарантируют также и единственность решений, что ясно из результатов этого пункта.

ПримеР 1.7.16. Пусть $\Omega=\mathbb{R}^{d}, A=\mathrm{I}, b^{i} \in L_{\mathrm{loc}}^{p}\left(\mathbb{R}^{d}\right)$ и

$$
\limsup _{|x| \rightarrow \infty}|x|^{\gamma-1}[2(\gamma-1)+d+\langle b(x), x\rangle]=-\infty
$$

для некоторого $\gamma \geqslant 1$. Тогда $\# \mathscr{M}_{\mathrm{ell}}^{A, b}=1$.

ПримеР 1.7.17. Предположим, что $\Omega=\mathbb{R}^{1}, A$ локально абсолютно непрерывно и положительно, функция $b$ локально интегрируема по Лебегу. Если

$$
\int_{-\infty}^{0} \frac{1}{\sqrt{A(s)}} d s=\infty, \quad \int_{0}^{+\infty} \frac{1}{\sqrt{A(s)}} d s=\infty,
$$

то $\# \mathscr{M}_{\mathrm{ell}}^{A, b} \leqslant 1$.

ЗАмечАниЕ 1.7.18. Пусть $A$ - положительная локально абсолютно непрерывная функция на $\mathbb{R}^{1}$, причем (1.7.2) не выполнено. Тогда можно показать, что существует локально интегрируемая функция $b$, для которой $\# \mathscr{M}_{\mathrm{ell}}^{A, b}=\infty$.

ЗАмечАниЕ 1.7.19. Пусть $\Omega=\mathbb{R}^{1}, A(x)=1$ и $b(x)=-2 x-6 e^{x^{2}}$. Тогда $\mathscr{M}_{\mathrm{ell}}^{A, b}=\{\mu\}$, где $\mu(d x)=\pi^{-1 / 2} e^{-x^{2}} d x$, но, согласно [50; пример 1.12], оператор $\left(L_{A, b}, C_{0}^{\infty}\left(\mathbb{R}^{1}\right)\right)$ не является существенно $m$-диссипативным на $L^{1}\left(\mathbb{R}^{1}, \mu\right)$. Значит, обратное утверждение к теореме 1.7 .2 неверно в случае $d=1$.

В изложенных результатах о единственности предполагалась соболевская дифференцируемость $a^{i j}$. Не исследован случай общего невырожденного измеримого коэффициента диффузии. Например, не исследована единственность 
решений уравнения $L_{A, b}^{*} \mu=0$ с равномерно ограниченными борелевскими $A$, $A^{-1}, b$. Стоит отметить, что если коэффициенты $a^{i j}$ и $b^{i}$ локально гёльдеровы, $\operatorname{det} A>0$, а вероятностная мера $\mu$ инвариантна для полугруппы $\left(T_{t}^{\mu}\right)_{t \geqslant 0}$, ассоциированной с $L_{A, b}$, то она имеет плотность, локально равномерно отделенную от нуля, и потому является единственной инвариантной мерой (см., например, [77; §8.1]).

Остановимся кратко на так называемом симметричном случае, т. е. случае $b=\beta_{A, \mu}$ (или $b=A \beta_{\mu}$ для операторов дивергентного вида). Если $A=\mathrm{I}$, то $b$ - это просто $\nabla \varrho / \varrho$. Этот случай привлек особое внимание в литературе, поскольку оператор $L=L_{\mathrm{I}, b}$ оказывается симметричным неположительным. Помимо $L^{1}$-единственности этого оператора, интенсивно изучалась также его существенная самосопряженность на $C_{0}^{\infty}(\Omega)$. Различные результаты можно найти в работах [6], [90]-[94], [49], [51], [50] и в имеющихся там ссылках. Отметим лишь, что, как показано в $[6]$, если $\Omega=\mathbb{R}^{d}$ и $A=\mathrm{I}$, то наше обычное предположение $|b| \in L_{\mathrm{loc}}^{p}\left(\mathbb{R}^{d}\right)$ при $p>d$ достаточно для существенной самосопряженности (если $d>3$, то, как показано в [94], достаточным оказывается несколько более слабое локальное условие; например, в терминах $L_{\mathrm{loc}}^{p}$ достаточно иметь $p=d$ ). Дальнейшие усиления получены в [93], где установлен следующий результат. Пусть плотность $\varrho \geqslant 0$ такова, что $\sqrt{\varrho} \in W_{\mathrm{loc}}^{2,1}\left(\mathbb{R}^{d}\right)$ и $\nabla \varrho / \varrho \in L_{\text {loc }}^{4}\left(\mathbb{R}^{d}, \varrho d x\right)$. Предположим дополнительно, что для каждого шара $B_{0}$ найдется такое $\varepsilon>0$, что

$$
\sup _{B \subset B_{0}}\left(|B|^{-1} \int_{B} \varrho(x)^{1+\varepsilon} d x\right)\left(|B|^{-1} \int_{B} \varrho(x)^{-1-\varepsilon} d x\right)<\infty,
$$

где супремум берется по всем шарам $B \subset B_{0}$ и $|B|$ - объем $B$. Тогда оператор $L_{\mathrm{I}, b}$ с $b=\nabla \varrho / \varrho$ существенно самосопряжен на $C_{0}^{\infty}\left(\mathbb{R}^{d}\right) \subset L^{2}(\varrho d x)$. Все еще неизвестно, достаточно ли лишь одно условие

$$
\frac{\nabla \varrho}{\varrho} \in L_{\mathrm{loc}}^{4}\left(\mathbb{R}^{d}, \varrho d x\right)
$$

(но такое глобальное условие достаточно). В [95] показано, что в симметричном случае условия (A1) и (A2) из (1.5.2) достаточны для существенной самосопряженности $L_{A, b}$ на $C_{0}^{\infty}(M)$, если риманово многообразие $M$ полно относительно метрики, порожденной $A^{-1}$. В [95] показано также, что при таких же предположениях для симметричного оператора $L_{A, b}$ имеет место более сильная $L^{p}$-единственность.

Пусть теперь в симметричном случае мы имеем $A=\mathrm{I}$. Предположим, что $b$ есть фиксированная борелевская $d x$-версия отображения $\nabla \varrho_{0} / \varrho_{0}$ для некоторой вероятностной плотности $\varrho_{0} \in W_{\mathrm{loc}}^{1,1}\left(\mathbb{R}^{d}\right)$, где мы полагаем $\nabla \varrho_{0} / \varrho_{0}:=0$ на множестве $\left\{\varrho_{0}=0\right\}$, т. е. берется $d x$-версия логарифмического градиента $\beta^{\mu_{0}}$ меры $\mu_{0}:=\varrho_{0} d x$. Тогда $\mu_{0} \in \mathscr{M}_{\mathrm{ell}}^{\mathrm{I}, b}$. Уточнение о характере версии связано с тем, что мера $\mu_{0}$ не обязана быть эквивалентной мере Лебега, поэтому $\mu_{0}$-версия $b$ может не быть $d x$-версией. Ясно, что $|b| \in L_{\text {loc }}^{1}\left(\mu_{0}\right)$. В этом случае имеется следующий результат (см. [7]) с гораздо более слабым локальным условием на $b$, чем в теореме 1.7.1, но опять нам требуется глобальное условие (1.5.1). 
ТЕОРема 1.7.20. Пусть b имеет указанный вид $и|b| \in L_{\mathrm{loc}}^{2}\left(\mu_{0}\right)$.

(i) Пусть $\mu \in \mathscr{M}_{\mathrm{ell}}^{\mathrm{I}, b}$, причем $|b| \in L_{\mathrm{loc}}^{2}(\mu)$ u $\varrho:=d \mu / d x$ удовлетворяет (1.5.1). Тогда

$$
\frac{\nabla \varrho}{\varrho}=b \quad \mu-n .6 .
$$

и оператор $\left(L, C_{0}^{\infty}\left(\mathbb{R}^{n}\right)\right)$ симметричен на $L^{2}(\mu)$.

(ii) $E c л и|b| \in L_{\mathrm{loc}}^{1}(U)$ для некоторого связного открытого множества $U$ в $\mathbb{R}^{d}$, дополнение которого имеет лебеговскую меру нуль, то $\mu_{0}-$ единственная мера $\mu \in \mathscr{M}_{\mathrm{ell}}^{\mathrm{I}, b}$, для которой $|b| \in L_{\mathrm{loc}}^{2}(\mu) u \varrho=d \mu / d x$ удовлетворяem (1.5.1).

Утверждение (i) дает условия, при которых инфинитезимально инвариантные меры являются симметричными. Однако возникает такой вопрос: пусть вероятностная мера $\mu$ удовлетворяет уравнению $L_{\mathrm{I}, b}^{*}=0$, где $b=\nabla V$ для некоторой функции $V \in C^{\infty}\left(\mathbb{R}^{d}\right)$; верно ли, что $b$ совпадает с логарифмическим градиентом $\mu$ и тогда $\mu=c \exp (V) d x$ ? Как показывает следующий пример, предложенный С. В. Шапошниковым, это неверно без дополнительных предположений.

ПримеР 1.7.21. Возьмем следующую гладкую функцию на прямой:

$$
V(x, y)=-\left(\ln \left(1+x^{2}\right)+\ln \left(1+y^{2}\right)+x+\frac{x^{3}}{3}+y+\frac{y^{3}}{3}\right) .
$$

Тогда мера $\mu$ с плотностью $\varrho(x)=\left(1+x^{2}\right)^{-1}\left(1+y^{2}\right)^{-1}$ удовлетворяет уравнению $L_{\mathrm{I}, b}^{*} \mu=0$, где $b=\nabla V$. В самом деле, это уравнение можно записать в виде уравнения на плотность $\operatorname{div}(\nabla \varrho-\varrho b)=0$, а для указанной функции $\varrho$ находим $\nabla \varrho-\varrho b=\left(\left(y^{2}+1\right)^{-1},\left(x^{2}+1\right)^{-1}\right)$, что очевидным образом имеет нулевую дивергенцию.

Однако можно показать, что ответ положителен, если $|\nabla V| \in L^{1}(\mu)$ или $\exp (V) \in L^{1}\left(\mathbb{R}^{d}\right)$.

Про проблемы единственности см. также [58], [96].

1.8. Глобальные свойства плотностей. Перейдем к некоторым глобальным оценкам, связанным с регулярностью инвариантных мер. В отличие от примера 1.2.15, условия на порядок интегрируемости сноса могут быть значительно ослаблены в случае глобальной интегрируемости. Следующий результат получен в [5]. Предположим, что отображение $A$ со значениями в пространстве положительных симметричных матриц равномерно ограничено, равномерно липшицево и существует такое $\alpha>0$, что

$$
\langle A(x) h, h\rangle \geqslant \alpha\langle h, h\rangle \quad \forall x, h \in \mathbb{R}^{d} .
$$

Положим

$$
a:=\left(a^{1}, \ldots, a^{d}\right), \quad a^{j}:=\sum_{i=1}^{d} \partial_{i} a^{i j} .
$$

ТеОрема 1.8.1. Пусть $\mu$ - борелевская вероятностная мера на $\mathbb{R}^{d}, b$ - борелевское векторное поле, $|b| \in L^{2}(\mu)$ u $L_{A, b}^{*} \mu=0$. Тогда: 
1) $\mu=\varrho d x$, где $\varrho=\varphi^{2} u \varphi \in W^{2,1}\left(\mathbb{R}^{d}\right)$, в частности, $\varrho \in L^{d /(d-1)}\left(\mathbb{R}^{d}\right)$;

2) выполнено неравенство

$$
\frac{1}{4} \int_{\mathbb{R}^{d}}\left|\frac{\nabla \varrho}{\varrho}\right|^{2} \varrho d x=\int_{\mathbb{R}^{d}}|\nabla \varphi|^{2} d x \leqslant \frac{1}{4 \alpha^{2}} \int|b+a|^{2} d \mu
$$

3) отображение $\nabla \varrho / \varrho$ совпадает $\mu$-n. в. с ортогональной проекцией векторного поля $A^{-1}(b-a)$ на замъкание множества $\left\{\nabla u \mid u \in C_{0}^{\infty}\left(\mathbb{R}^{d}\right)\right\}$ в пространстве $L^{2}\left(\mu, \mathbb{R}^{d}\right)$, наделенном скалярным произведением $\langle\cdot, \cdot\rangle_{2}$, заданным формулой

$$
\langle F, G\rangle_{2}:=\int_{\mathbb{R}^{d}}\langle A F, G\rangle d \mu .
$$

В частности, если $A=\mathrm{I}$, то эти утверждения верны с $\alpha=1$ и $а=0$.

СлЕДСТвИЕ 1.8.2. Пусть вероятностная мера $\mu$ на $\mathbb{R}^{d}$ удовлетворяет уравнению $L_{\mathrm{I}, b}^{*} \mu=0$, әде $|b| \in L^{2}(\mu)$. Оператор $L_{\mathrm{I}, b}$ на области $C_{0}^{\infty}\left(\mathbb{R}^{d}\right)$ в $L^{2}(\mu)$ симметричен в точности тогда, когда $b$ совпадает $\mu$-n. в. с логарифмическим градиентом $\mu$.

Эта теорема увеличивает глобальную интегрируемость $\varrho$, но очевидным образом не может обеспечить равномерную ограниченность $\varrho$. Последнее будет получено ниже с помощью включения $\varrho \in W^{p, 1}\left(\mathbb{R}^{d}\right)$ при $p>d$ при дополнительных предположениях, но уже сейчас мы можем использовать локальные оценки из п. 1.2, чтобы установить равномерную оценку на $\varrho$.

Теорема 1.8.3. Пусть $\mu$ - борелевская вероятностная мера на $\mathbb{R}^{d} u$ $L_{A, b}^{*} \mu=0$, где А удовлетворяет условиям, указанным перед теоремой 1.8.1, u b удовлетворяет следующему условию с некоторым $p>d$ :

$$
\text { либо } \sup _{x \in \mathbb{R}^{d}}\|b\|_{L^{p}(B(x, 1))}<\infty, \quad \text { либо } \sup _{x \in \mathbb{R}^{d}}\|b\|_{L^{p}(B(x, 1), \mu)}<\infty .
$$

Тогда непрерывная версия @ плотности меры $\mu$ равномерно ограничена. Если $|b| \in L^{p}(\mu)$, mo $\varrho \in W^{p, 1}\left(\mathbb{R}^{d}\right)$.

ДокАЗАТЕЛЬство. Из п. 1.2 следует, что максимум $\varrho$ на $B(z, 1)$ оценивается числом, зависящим только от $\sup _{x}\left[\|A(x)\|+\left\|A(x)^{-1}\right\|\right]$, постоянной Липшица для $A$ и $\|b\|_{L^{p}(B(z, 1))}$ (или $\|b\|_{L^{p}(B(z, 1), \mu)}$ во втором случае), значит, мажорируется числом, не зависящим от $z$. Если $|b| \in L^{p}(\mu)$, то $W^{p, 1}$-норма $\varrho$ на кубе $K$ с единичным ребром оценивается через $C\left(\|\varrho\|_{L^{1}(Q)}+\|b\|_{L^{p}(Q, \mu)}\right)$, где $Q$ - куб с тем же центром и удвоенным ребром, причем $C$ не зависит от центра. Поэтому $W^{p, 1}$-норма $\varrho$ конечна на всем пространстве. Теорема доказана.

Последнее утверждение будет усилено ниже.

Следующий глобальный результат об эллиптической регулярности использует некоторое равномерное локальное условие на $A$. При $a^{i j} \in W_{\mathrm{loc}}^{1,1}\left(\mathbb{R}^{d}\right)$ положим

$$
\Theta_{A}(x):=\sum_{j=1}^{d}\left|\sum_{i=1}^{d} \partial_{x_{i}} a^{i j}(x)\right|
$$


Для данных $p>1$ и $\gamma>0$ положим

$$
q=q(d, p, \gamma)= \begin{cases}d & \text { при } p>\frac{d}{d-1}, \\ d+\gamma & \text { при } p=\frac{d}{d-1}, \\ p^{\prime}=\frac{p}{p-1}(>d) & \text { при } p<\frac{d}{d-1} .\end{cases}
$$

Будем говорить, что $A$ удовлетворяет условию $(\mathrm{C} 1)$ для $p>1$, если мы имеем $a^{i j} \in W_{\text {loc }}^{1,1}\left(\mathbb{R}^{d}\right)$ и

$$
\lim _{r \rightarrow 0} \sup _{z \in \mathbb{R}^{d}} \int_{B(z, r)} \Theta_{A}^{q}(x) d x=0,
$$

где $q$ определено выше (в случае $p=d /(d-1)$ это равенство должно быть выполнено с $q=d+\gamma$ для некоторого $\gamma>0)$.

Заметим, что это условие слабее, чем

$$
\lim _{r \rightarrow 0} \sup _{z \in \mathbb{R}^{d}} \int_{B(z, r)} \widetilde{\Theta}_{A}^{q}(x) d x=0,
$$

где

$$
\widetilde{\Theta}_{A}(x):=\sum_{j=1}^{d} \sum_{i=1}^{d}\left|\partial_{x_{i}} a^{i j}(x)\right| .
$$

Ясно, что если имеется такое $p_{0}>d>1$, что

$$
\sup _{z \in \mathbb{R}^{d}} \int_{B(z, 1)} \sum_{i, j=1}^{d}\left|\nabla a^{i j}(x)\right|^{p_{0}} d x<\infty,
$$

то $A$ удовлетворяет условию (C1) (как и (1.8.2)) для всех $p \in\left(1, p_{0}\right)$ и равномерно непрерывно (даже равномерно гёльдерово) на всем $\mathbb{R}^{d}$. В частности, оба свойства выполнены, если $A$ равномерно липшицево.

Стоит заметить, что хотя в большинстве наших результатов мы предполагаем, что $a^{i j} \in W_{\text {loc }}^{p, 1}\left(\mathbb{R}^{d}\right)$, а тогда можно записать $\mathscr{L}_{A, b}$ как $L_{A, b_{0}}$ для сноса $b_{0}^{i}:=b^{i}+\partial_{x_{j}} a^{i j}$, случай $\mathscr{L}_{A, b}$ не всегда сводится к случаю $L_{A, b}$, поскольку условия глобальной интегрируемости на $|b|$ и $\left|\nabla a^{i j}\right|$ различны. В некоторых ситуациях легче иметь дело с операторами дивергентного вида, в иных случаях стандартный вид удобнее. В случае многообразия обычно дивергентная форма операторов приводит к более естественным геометрическим объектам. Видимо, наиболее естественная постановка обсуждаемых задач должна апеллировать к геометрии, связанной с $A$, и весовым пространствам Соболева. Однако соответствующая техника, в частности, теоремы вложения, менее развита, чем классическая теория Соболева.

Tеорема 1.8.4. Пусть мера $\mu \in \mathscr{M}\left(\mathbb{R}^{d}\right)$ такова, что

$$
L_{A, b}^{*} \mu=\nu \in W^{p,-1}\left(\mathbb{R}^{d}\right)
$$

для некоторого $p \in(1, d /(d-1)),|b| \in L^{1}(|\mu|)$. Предположим, что А равномерно непрерывно и $c_{1} \cdot \mathrm{I} \leqslant A(x) \leqslant c_{2} \cdot \mathrm{I}$ для некоторых постоянных $c_{1}, c_{2}>0$. Тогда $\mu$ имеет плотность из $L^{r}\left(\mathbb{R}^{d}\right)$ для каждого $r \in[1, p]$. 
$B$ случае $\mathscr{L}_{A, b}$ это же верно при дополнительном предположении, что $a^{i j} \in$ $W_{\mathrm{loc}}^{1,1}\left(\mathbb{R}^{d}\right)$ для всех $i, j$ и $\partial_{x_{i}} a^{i j} \in L^{1}(|\mu|)$ для всех $j$.

Следующий результат обобщает теорему 3.1 работы [97] и частично обобщает результат из [5]. Мы накладываем более слабые предположения, чем в [97], где $A^{-1}$ ограничено и $|b| \in L^{2}(\mu)$ (кроме того, в [97] сделаны те же локальные предположения, что и ниже, вместе с некоторым условием, которое немного сильнее (1.8.4)); по сравнению с [5] (где $A$ равномерно липшицево, $A$ и $A^{-1}$ равномерно ограничены, $\left.|b| \in L^{2}(\mu)\right)$, мы ослабляем предположения на $A$, но добавляем новое локальное условие на $b$. Это новое условие не нужно, если заранее известно, что $\mu$ имеет локально ограниченную плотность из $W_{\mathrm{loc}}^{2,1}\left(\mathbb{R}^{d}\right)$. Следует отметить, что в отличие от большинства других результатов этого пункта, данная теорема имеет дело с вероятностными мерами и неверна для знакопеременных мер.

ТеОрема 1.8.5. Предположим, что мера $\mu \in \mathscr{P}\left(\mathbb{R}^{d}\right)$ удовлетворяет уравнению $\mathscr{L}_{A, b}^{*} \mu=0$, где отображение $A$ непрерьвно, $\operatorname{det} A>0, a^{i j} \in W_{\mathrm{loc}}^{p, 1}\left(\mathbb{R}^{d}\right)$ при некотором $p>d,|b| \in L_{\mathrm{loc}}^{p}(\mu)$. Предположим также, что $\left|A^{-1 / 2} b\right| \in$ $L^{2}(\mu)$ и что

$$
\liminf _{r \rightarrow \infty} \int_{r \leqslant|x| \leqslant 2 r}\left[r^{-2}\|A(x)\|+r^{-1} \Theta_{A}(x)\right] \mu(d x)=0 .
$$

Тогда $\mu$ имеет такую плотность $\varrho \in W_{\mathrm{loc}}^{2,1}\left(\mathbb{R}^{d}\right)$, что

$$
\int_{\mathbb{R}^{d}}\left|\frac{\sqrt{A} \nabla \varrho}{\varrho}\right|^{2} d \mu \leqslant \int_{\mathbb{R}^{d}}\left|A^{-1 / 2} b\right|^{2} d \mu .
$$

$B$ частности, при дополнительном предположении $A \geqslant \varepsilon \cdot \mathrm{I}, \varepsilon>0$, имеем $\sqrt{\varrho} \in W^{2,1}\left(\mathbb{R}^{d}\right), \varrho \in L^{d /(d-2)}\left(\mathbb{R}^{d}\right)$ nри $d>2 u \varrho \in L^{s}\left(\mathbb{R}^{d}\right)$ для всех $s \in[1, \infty)$ npu $d=2$.

ЗАмечАниЕ 1.8.6. (i) Условие (1.8.4) выполнено, если

$$
\left|\nabla a^{i j}(x)\right| \leqslant C_{0}+C_{1}|x|
$$

Если известно, что $\mu$ имеет конечный первый момент, т. е. $|x| \in L^{1}(\mu)$, то допустим квадратичный рост $\left|\nabla a^{i j}\right|$.

(ii) Условие (1.8.4) можно заменить предположением, что для некоторого $r>0$ мы имеем

$$
\liminf _{R \rightarrow \infty} \int_{R \leqslant|x| \leqslant R+r}\left[\|A(x)\|+\Theta_{A}(x)\right] \mu(d x)=0 .
$$

Это условие слабее по части $\Theta_{A}$, но сильнее по части $\|A\|$; для равномерно ограниченного $A$ оно слабее.

(iii) Если $A$ равномерно ограничено и удовлетворяет (C1) с (1.8.1), то (1.8.4) обеспечивается предположением, что $\liminf _{r \rightarrow \infty} r^{d-1} \mu(\{|x| \geqslant r\})=0$, которое выполнено, например, если $|x|^{d-1} \in L^{1}(\mu)$. Последнее можно эффективно проверять в терминах $A$ и $b$ с помощью метода функций Ляпунова. 
Оценка (1.8.5) может рассматриваться как оценка

$$
\int\left\langle\frac{\nabla \varrho}{\varrho}, \frac{\nabla \varrho}{\varrho}\right\rangle d \mu \leqslant \int\langle b, b\rangle d \mu
$$

относительно римановой геометрии, порожденной $A$. Такая оценка действительно получена в [24], [25] для широкого класса римановых многообразий (про диффузионные операторы на многообразиях см. также [98]).

Теорема 1.8.7. Пусть $M$ - такое риманово многообразие с римановой мерой объема $\lambda$, что кривизна Риччи ограничена снизу и римановы объемы шаров всякого фиксированного положительного радиуса отделены от нуля. Пусть $\mu$ - такая борелевская вероятностная мера на $M$, что $L^{*} \mu=0$, где $L f=$ $\Delta f+\langle b, \nabla f\rangle u|b| \in L^{2}(\mu)$. Тогда $\mu=\varrho \cdot \lambda$, где $\sqrt{\varrho} \in W^{2,1}(M) u$

$$
\int_{M} \frac{|\nabla \varrho|^{2}}{\varrho^{2}} d \mu \leqslant \int_{M}|b|^{2} d \mu .
$$

Если вместо $|b| \in L^{2}(\mu)$ мы имеем $|b| \in L^{2}(\lambda)$, то

$$
\int_{M} \frac{|\nabla \varrho|^{2}}{\varrho^{2}} d \lambda \leqslant \int_{M}|b|^{2} d \lambda .
$$

На самом деле технические условия на $M$, использованные в [24], даже шире и выражены в терминах полугруппы уравнения теплопроводности. Однако, как указано в [25; замечание 2.5 (ii)], эта оценка может быть неверна для общих римановых многообразий, даже если $b=0$. А именно, существуют полные связные римановы многообразия, на которых есть непостоянные положительные интегрируемые гармонические функции; такая функция задает меру, удовлетворяющую нашему уравнению с $b=0$, а тогда оценка выше неверна для нее. В ситуации теоремы 1.8.5 нам неизвестно, выполняется ли естественная оценка (1.8.5) без всяких дополнительных локальных условий на $b$ и без (1.8.4). Однако есть важный частный случай, когда (1.8.4) не требуется.

Tеорема 1.8.8. Пусть А непреръвно, $\operatorname{det} A>0, a^{i j} \in W_{\mathrm{loc}}^{p, 1}\left(\mathbb{R}^{d}\right),|b| \in$ $L_{\mathrm{loc}}^{p}\left(\mathbb{R}^{d}\right)$ при некотором $p>d$. Предположим, что существует такал квазикомпактная функиия $V \in W_{\mathrm{loc}}^{2,2}\left(\mathbb{R}^{d}\right)$, что

$$
\mathscr{L}_{A, b} V(x) \rightarrow-\infty \quad \text { npu }|x| \rightarrow+\infty .
$$

Предположим также, что данъ такие числа $c_{1}, c_{2}>0$, что

$$
\mathscr{L}_{A, b} V \leqslant c_{1}-c_{2}\left|A^{-1 / 2} b\right|^{2}
$$

вне некоторого шара. Тогда найдется такая мера $\mu \in \mathscr{P}\left(\mathbb{R}^{d}\right)$ с положительной плотностью $\varrho \in W_{\text {loc }}^{p, 1}\left(\mathbb{R}^{d}\right)$, что $\mathscr{L}_{A, b}^{*} \mu=0 u|\sqrt{A} \nabla \varrho|^{2} / \varrho \in L^{1}\left(\mathbb{R}^{d}\right)$.

Если, кроме того, существует положительная борелевская функиия $\theta$ на $[0,+\infty)$, для которой $\lim _{t \rightarrow \infty} \theta(t)=+\infty u$

$$
\mathscr{L}_{A, b} V \leqslant c_{1}-c_{2} \theta\left(\left|A^{-1 / 2} b\right|\right)\left|A^{-1 / 2} b\right|^{2}
$$

вне некоторого шара, то $\left|A^{-1 / 2} b\right| \in L^{2}(\mu)$ и выполнено (1.8.5). 
Теорема 1.8.9. Пусть мера $\mu \in \mathscr{M}\left(\mathbb{R}^{d}\right)$ такова, что $\mathscr{L}_{A, b}^{*} \mu=\nu$. Предположим, что

(a) $A \geqslant \varepsilon \mathrm{I}$ при некотором $\varepsilon>0$,

$$
a^{i j} \in W_{\mathrm{loc}}^{\alpha, 1}\left(\mathbb{R}^{d}\right) \text { и либо }|b| \in L_{\mathrm{loc}}^{\alpha}\left(\mathbb{R}^{d}\right), \text { либо }|b| \in L_{\mathrm{loc}}^{\alpha}(|\mu|) \text {, где } \alpha>d,
$$

(b) $|b|$, trace $A \in L^{\beta}(|\mu|)$, где $\beta>1$,

(c) А удовлетворяет условию (C1) выше для $\beta$ из (b) и равномерно непрерывно.

Предположим также, что плотность $\varrho$ мерь $\mu$ входит в $L^{\beta_{0}}\left(\mathbb{R}^{d}\right)$ при некотором $\beta_{0}>1$, что автоматически выполнено в (i)-(iii) ниже, если А ограничено и $\partial_{x_{i}} a^{i j} \in L^{\beta}(|\mu|)$ для каждого $j$.

(i) Пусть $1<\beta<d u \nu \in W^{\theta,-1}$ для всех $\theta \in(1, d /(d-\beta+1))$. Тогда $\varrho \in W^{r, 1}\left(\mathbb{R}^{d}\right)$ для всех $r \in(1, d /(d-\beta+1))$. Более того, если н неотрицательна, то это же верно для $r=d /(d-\beta+1)$.

(ii) Пусть $\beta=d u \nu \in W^{\theta,-1}\left(\mathbb{R}^{d}\right)$ для всех $\theta \in(1, d)$. Тогда $\varrho \in W^{r, 1}\left(\mathbb{R}^{d}\right)$ для всех $r \in(1, d)$.

(iii) Пусть $d<\beta \leqslant \alpha u \nu \in W^{\theta,-1}\left(\mathbb{R}^{d}\right)$ для всех $\theta \in(1, \beta]$. Тогда имеем $\varrho \in W^{r, 1}\left(\mathbb{R}^{d}\right)$ для всех $r \in(1, \beta]$. В частности, $\varrho \in L^{\infty}\left(\mathbb{R}^{d}\right)$.

Утверждения (i)-(iii) верны в случае $L_{A, b}$, если дополнительно для каждого $j$ мы имеем включение $\partial_{x_{i}} a^{i j} \in L^{\beta}(|\mu|)$.

СлеДСтвие 1.8.10. Пусть $\mu \in \mathscr{P}\left(\mathbb{R}^{d}\right)$ u $\mathscr{L}_{A, b}^{*} \mu=0$. Предположим, что есть такое $\alpha>d$, что $|b|$, trace $A \in L^{\alpha}(\mu)$, $a^{i j} \in W_{\mathrm{loc}}^{\alpha, 1}\left(\mathbb{R}^{d}\right)$ равномерно непрерывны и выполнены (C1) $u$ (1.8.4), $A \geqslant \varepsilon \mathrm{I}$, где $\varepsilon>0$. Тогда $\mu=\varrho d x$, где $\varrho \in W^{\alpha, 1}\left(\mathbb{R}^{d}\right)$, в частности, $\varrho \in L^{\infty}\left(\mathbb{R}^{d}\right)$.

В частности, заключение верно, если выполнено (1.8.3) и

$$
A \geqslant \varepsilon \mathrm{I}, \quad|b| \in L^{\alpha}(\mu), \quad \operatorname{trace} A \in L^{\alpha}(\mu), \quad(1+|x|)^{-1} \Theta_{A} \in L^{1}(\mu) .
$$

Случай $\beta=1$ в теореме выше пока не исследован. В частности, неизвестно, верно ли, что $\varrho \in W^{1,1}\left(\mathbb{R}^{d}\right)$, т. е. $|\nabla \varrho / \varrho| \in L^{1}(\mu)$, если $|b| \in L^{1}(\mu)$ и $\mu$ - вероятностная мера, удовлетворяющая уравнению $L_{\mathrm{I}, b}^{*} \mu=0$. Если $|b| \in L^{2}(\mu)$, то это верно ввиду теоремы 1.8.1. Кроме того, неизвестно, верно ли в этой же ситуации, что $|\nabla \varrho / \varrho| \in L^{p}(\mu)$, если $|b| \in L^{p}(\mu)$ и $p \neq 2$. Дополнительные достаточные условия для этого указаны в п. 1.9. Поэтому без каких-либо дополнительных условий типа наличия функций Ляпунова или оценок на коэффициенты включение $\varrho \in W^{1,1}\left(\mathbb{R}^{d}\right)$ установлено пока лишь в предположении, что $|b| \in L^{2}(\mu)$.

Ясно, что $\varrho$ может не входить в класс $W^{p, 2}$, если не требовать некоторую регулярность $b$. Следующая теорема обобщает теорему 4.7 в [97], где использованы несколько более сильные условия на $A$.

Tеорема 1.8.11. Предположим, что $\alpha \geqslant 2 d, A \geqslant \varepsilon \mathrm{I} c \varepsilon>0, a^{i j}, b^{i} \in$ $W_{\mathrm{loc}}^{\alpha, 1}\left(\mathbb{R}^{d}\right), A$ равномерно непрерьвно и удовлетворяет условию (C1) для $\alpha$. Пусть $\mu \in \mathscr{P}\left(\mathbb{R}^{d}\right)$ удовлетворяет уравнению $\mathscr{L}_{A, b}^{*} \mu=\nu$, где $|b|$, trace $A \in L^{\alpha}(\mu)$ $u \nu \in L^{r}\left(\mathbb{R}^{d}\right)$ для всех $r \in(1, \alpha]$. Предположим тажже, что $\operatorname{div} b \in L^{\alpha}(\mu)$ $u\left|\nabla a^{i j}\right| \in L^{\theta}(\mu)$, где $\theta \geqslant \max \left(2 d^{2}, \alpha\right)$. Тогда $\mu=\varrho d x$, где $\varrho \in W^{r, 2}\left(\mathbb{R}^{d}\right)$ для всех $r \in(1, \alpha / 2)$. Если $\alpha>2 d$, mо $\varrho \in W^{\alpha / 2,2}\left(\mathbb{R}^{d}\right) u|\nabla \varrho| \in L^{\infty}\left(\mathbb{R}^{n}\right)$.

Если $a^{i j},\left|\nabla a^{i j}\right| \in L^{\infty}\left(\mathbb{R}^{d}\right)$, то эти утверждения верны для всякого решения $\mu$ из класса всех мер ограниченной вариачии. 
Теперь обратимся к поточечным оценкам решений. Идея проста: чтобы показать, что $|\varrho(x)| \leqslant C \Psi(x)$ для некоторой положительной функции $\Psi$, следует рассмотреть меру $\mu_{0}$ с плотностью $\varrho / \Psi$ и проверить, что эта мера удовлетворяет уравнению рассмотренного в теореме 1.8.9 типа. Эта идея была использована в [97] в случае экспоненциальных функций. Относительно дальнейшего развития этого метода мы отсылаем к работе [99], где были получены следующие результаты. Случай (iii) примера 1.8.13 ниже дает оценку из [97] при немного более слабых предположениях.

Теорема 1.8.12. Предположим, что вероятностная мера $\mu$ удовлетворяет уравнению $\mathscr{L}_{A, b}^{*} \mu=0$, где для $A$ и b выполнены условия (a), (b) теоремъ 1.8.9 при некотором $\alpha=\beta>d$. Пусть $\Phi \in W_{\mathrm{loc}}^{1,1}\left(\mathbb{R}^{d}\right)-$ такая положительная функиия, что для некоторого $\theta>d$ и всех $j=1, \ldots, d$ мы имеем

$$
\Phi \in L^{1}(\mu), \quad|\nabla \Phi| \in L^{\theta}(\mu), \quad \partial_{x_{i}} a^{i j} \in L^{d}(\mu) .
$$

Тогда для плотности @меры $\mu$ выполнена оценка $\varrho(x) \leqslant C \Phi(x)^{-1}$ с некоторой постоянной $C$.

Сейчас мы увидим, что наличие полиномиальных или экспоненциальных моментов решений дает убывание плотностей на бесконечности соответствующего типа.

ПримеР 1.8.13. Предположим, что $\mu \in \mathscr{P}\left(\mathbb{R}^{d}\right)$ удовлетворяет уравнению $\mathscr{L}_{A, b}^{*} \mu=0$ и что $A$ равномерно липшицево, $A$ и $A^{-1}$ равномерно ограничены. Кроме того, предположим, что $|b| \in L^{p}(\mu)$ для некоторого $p>d$.

(i) Если $\Psi \in W_{\text {loc }}^{1,1}\left(\mathbb{R}^{d}\right)$ - положительная функция, для которой

$$
\Phi \in L^{1}(\mu), \quad|\nabla \Phi| \in L^{\theta}(\mu), \quad \theta>d,
$$

то $\varrho(x) \leqslant C \Phi(x)^{-1}$.

(ii) Пусть $k>1$ и $|x|^{r} \in L^{1}(\mu)$ для некоторого $r>(k-1) d$. Тогда, полагая $\Phi(x)=|x|^{k}$, получим $\varrho(x) \leqslant C|x|^{-k}$.

(iii) Предположим, что

$$
\exp \left(\alpha|x|^{\beta}\right) \in L^{1}(\mu), \quad|b(x)| \leqslant C_{0}+C_{1} \exp \left(\alpha_{0}|x|^{\beta}\right),
$$

где $\alpha, \beta, C_{0}, C_{1}>0, \alpha_{0}<\alpha / d$. Тогда для всякого $\kappa<\beta / d$ найдется такое число $C>0$, что $\varrho(x) \leqslant C \exp \left(-\kappa|x|^{\beta}\right)$.

Удивительно, что приведенные оценки, весьма грубые на первый взгляд, на самом деле достаточно точны. Далее мы увидим, что имеются нижние оценки такого же порядка.

Похожим образом получаются верхние оценки для $|\nabla \varrho|$.

ПреДЛОЖЕНИЕ 1.8.14. Предположим, что в теореме 1.8 .12 мы дополнительно имеем $\Phi \in W_{\mathrm{loc}}^{1,2}\left(\mathbb{R}^{d}\right)$ u

$$
\varrho|\nabla \Phi| \in L^{\infty}\left(\mathbb{R}^{d}\right), \quad|b|,|\nabla \Phi|, \partial_{x_{i}} a^{i j} \partial_{x_{j}} \Phi, L_{A} \Phi,|A \nabla \Phi| \in L^{r}(\mu), \quad r>2 d .
$$

Тогда $|\nabla \varrho(x)| \leqslant C \Phi(x)^{-1}$. 
ПримеР 1.8.15. Пусть $\mu \in \mathscr{P}\left(\mathbb{R}^{d}\right)$ удовлетворяет уравнению $\mathscr{L}_{A, b}^{*} \mu=0$, где $A$ равномерно липшицево, $A$ и $A^{-1}$ равномерно ограничены, причем $|b|, \operatorname{div} b \in L^{p}(\mu)$ для некоторого $p>2 d$.

(i) Пусть $\Phi(x)=|x|^{k}, k \geqslant 1$, и $|x|^{m} \in L^{1}(\mu)$, где $m>2 d(k-1)$. Тогда

$$
|\nabla \varrho(x)| \leqslant C(1+|x|)^{-k} .
$$

(ii) Пусть $\Phi(x)=\exp \left(K|x|^{\beta}\right)$ и $\exp \left(M|x|^{\beta}\right) \in L^{1}(\mu)$, где $M>2 d K$. Тогда

$$
|\nabla \varrho(x)| \leqslant C \exp \left(-K|x|^{\beta}\right) .
$$

Используя метод функций Ляпунова, можно дать конструктивные условия существования полиномиальных или экспоненциальных моментов меры $\mu$. Например, если $A(x) \leqslant \Lambda \mathrm{I}$ и $\langle b(x), x\rangle \leqslant-K<-\Lambda d$ вне некоторого шара, то, полагая $V(x)=\langle x, x\rangle^{\gamma}$ при $1<\gamma<1+(K-\Lambda d) / 2$, получим вне некоторого шар

$$
L_{A, b} V(x) \leqslant 2 \gamma\langle x, x\rangle^{\gamma-1}[\Lambda d+2(\gamma-1)+\langle b(x), x\rangle] \leqslant-\kappa|x|^{2 \gamma-2},
$$

где $\kappa>0$. Значит, $|x|^{2 \gamma-1} \in L^{1}(\mu)$. Более сильное убывание $\langle b(x), x\rangle$, например, оценка $\langle b(x), x\rangle \leqslant c_{1}-c_{2}|x|^{r}$, дает экспоненциальную интегрируемость (см. [10], [97]). Конечно, требуемая интегрируемость коэффициентов также может быть выведена из таких оценок, если известны некоторые оценки на эти коэффициенты. Стоит отметить, что некоторые условия на $A$, использованные выше, можно ослабить, применяя результаты из [37]-[39].

1.9. Нижние оценки. В этом пункте, следуя [22], где даны доказательства, мы обсудим нижние оценки плотностей решений эллиптических уравнений вида

$$
\mathscr{L}_{A, b}^{*} \mu=0
$$

для борелевских мер $\mu$ на $\mathbb{R}^{d}$, где $\mathscr{L}$ - эллиптический оператор второго порядка дивергентного вида

$$
\mathscr{L} \varphi(x):=\partial_{x_{i}}\left(a^{i j}(x) \partial_{x_{j}} \varphi(x)\right)+b^{i}(x) \partial_{x_{i}} \varphi(x) .
$$

Интерпретация этого уравнения обычная: функции $a^{i j}$ и $b^{i}$ должны быть интегрируемы на каждом компактном множестве в $\mathbb{R}^{d}$ относительно меры $\mu$ и для всех $\varphi \in C_{0}^{\infty}\left(\mathbb{R}^{d}\right)$ мы должны иметь равенство

$$
\int_{\mathbb{R}^{d}} \mathscr{L} \varphi d \mu=0
$$

Однако последнее может пониматься одним из двух следующих способов.

(I) Мы имеем $a^{i j} \in W_{\text {loc }}^{1,1}\left(\mathbb{R}^{d}\right)$, функции $a^{i j}, \partial_{x_{i}} a^{i j}$ и $b^{i}$ измеримы по Борелю и локально интегрируемы относительно $|\mu|$, причем

$$
\int_{\mathbb{R}^{d}}\left[a^{i j} \partial_{x_{i}} \partial_{x_{j}} \varphi+\partial_{x_{i}} a^{i j} \partial_{x_{j}} \varphi+b^{j} \partial_{x_{j}} \varphi\right] d \mu=0 .
$$


(II) Мера $\mu$ обладает плотностью $\varrho$ класса $W_{\text {loc }}^{1,1}\left(\mathbb{R}^{d}\right)$, причем функции $a^{i j} \partial_{x_{i}} \varrho$ и $b^{i} \varrho$ локально интегрируемы по Лебегу и

$$
\int_{\mathbb{R}^{d}}\left[-a^{i j} \partial_{x_{i}} \varrho \partial_{x_{j}} \varphi+b^{i} \partial_{x_{i}} \varphi \varrho\right] d x=0 .
$$

Ясно, что если коэффициенты $a^{i j}$ являются локально соболевскими и функции $\partial_{x_{i}} a^{i j} \varrho$ локально интегрируемы, то (1.9.3) можно записать как (1.9.2). Дивергентная форма операторов используется лишь для удобства формулировок; при наших стандартных предположениях на $A$ все основные результаты этого пункта могут быть легко переписаны для оператора $L_{A, b}$.

Как и выше, предположим, что $a^{i j}=a^{j i}$ и матрица $A(x)$ положительна.

В следующей теореме мы предположим, что неотрицательная локально ограниченная мера $\mu$ на $\mathbb{R}^{d}$ имеет такую плотность $\varrho$, что $\varrho \in W^{2,1}(U)$ для каждого шара $U \subset \mathbb{R}^{d}$. Пусть мера $\mu$ удовлетворяет уравнению (1.9.1) на $\mathbb{R}^{d}$ в смысле (1.9.3), т. е. в случае (II), где

$$
\mathscr{L}=\partial_{x_{i}}\left(a^{i j} \partial_{x_{j}}\right)+b^{i} \partial_{x_{i}},
$$

матричнозначное отображение $A=\left(a^{i j}\right)_{1 \leqslant i, j \leqslant d}$ измеримо, функции $\|A(x)\|$ и $\left\|A(x)^{-1}\right\|$ локально ограничены, коэффициент $b=\left(b^{i}\right)_{i \leqslant d}$ - измеримое локально ограниченное векторное поле. Напомним, что в случае, когда $a^{i j} \in W_{\mathrm{loc}}^{p, 1}\left(\mathbb{R}^{d}\right)$ и $b^{i} \in L_{\mathrm{loc}}^{p}\left(\mathbb{R}^{d}\right)$ при некотором $p>d$ и $A(x)^{-1}$ локально ограничено, всякое решение (1.9.1) в смысле (1.9.2) автоматически имеет плотность из $W_{\mathrm{loc}}^{p, 1}\left(\mathbb{R}^{d}\right)$ и, значит, удовлетворяет также (1.9.3).

Пусть $V$ - непрерывная возрастающая функция на $[0, \infty)$ и $V(0)>0$.

Теорема 1.9.1. Пусть $|b(x)| \leqslant V(|x| / \theta)$, где $\theta>1$. Положим

$$
\alpha(r):=\sup _{|x| \leqslant r}\left\|A(x)^{-1}\right\|, \quad \gamma(r):=\sup _{|x| \leqslant r}\|A(x)\| .
$$

Тогда существует такое положительное число $K(d)$, зависящее только от $d$, что непрерывная версия функиии @ удовлетворяет неравенству

$$
\varrho(x) \geqslant \varrho(0) \exp \left(-K(d)(\theta-1)^{-1} \alpha(\theta|x|)^{-1}(\gamma(\theta|x|)+V(|x|)|x|)\right) .
$$

В частности, если $\|A(x)\| \leqslant \gamma u\left\|A(x)^{-1}\right\| \leqslant \alpha$, то существует такое положительное число $K=K(d, \alpha, \gamma, \theta)$, что непрерывная версия функиии @ удовлетворяет неравенству

$$
\varrho(x) \geqslant \varrho(0) \exp (-K(1+V(|x|)|x|)) .
$$

ПРимеР 1.9.2. Предположим, что

$$
\sum_{i, j}\left|a^{i j}(x)\right|^{2} \leqslant \gamma^{2} \quad \text { и } \quad A(x) \geqslant \alpha \cdot \text { I для всех } x \in \mathbb{R}^{d} .
$$

Если для некоторых чисел $c_{1}, c_{2}>0$ при почти всех $x$ верна оценка

$$
|b(x)| \leqslant c_{1}|x|^{\beta}+c_{2},
$$


то существует такая постоянная $K$, что выполнено следующее неравенство:

$$
\varrho(x) \geqslant \varrho(0) \exp \left(-K\left(1+|x|^{\beta+1}\right)\right) .
$$

Если мы имеем

$$
\begin{gathered}
\sup _{x, i, j}\left[\|A(x)\|+\left\|A(x)^{-1}\right\|+\left|\nabla a^{i j}(x)\right|\right]<\infty, \\
|b(x)| \leqslant c_{1}|x|^{\beta}+c_{2}, \quad \limsup _{|x| \rightarrow \infty}|x|^{-\beta-1}(b(x), x)<0,
\end{gathered}
$$

то получаем следующую двустороннюю оценку:

$$
\exp \left(-K_{1}\left(1+|x|^{\beta+1}\right)\right) \leqslant \varrho(x) \leqslant \exp \left(-K_{2}\left(1+|x|^{\beta+1}\right)\right) .
$$

Верхняя оценка выполнена, если

$$
\begin{gathered}
\exp \left(M|x|^{\beta}\right) \in L^{1}(\mu), \quad|b(x)| \leqslant C_{0}+C_{1} \exp \left(M_{0}|x|^{\beta}\right), \quad 0 \leqslant M_{0}<d^{-1} M \\
\langle b(x), x\rangle \leqslant c_{0}-c_{1}|x|^{\beta}, \quad c_{1}>M \beta \sup _{x}\|A(x)\|,
\end{gathered}
$$

где $M>0$ достаточно мало. Например, если $A=\mathrm{I}$ и $b^{i}(x)=x_{i}$, то мера с плотностью $\varrho(x)=\exp \left(-|x|^{2} / 2\right)$ есть решение. Вышеупомянутые результаты обеспечивают оценку

$$
\exp \left(-K_{1}\left(1+|x|^{2}\right)\right) \leqslant \varrho(x) \leqslant \exp \left(-K_{2}\left(1+|x|^{2}\right)\right)
$$

с некоторыми числами $K_{1}, K_{2}>0$, что дает достаточно адекватное описание убывания на бесконечности, хотя и не дает точной асимптотики.

Следует отметить, что предположение, что $\lim \sup _{|x| \rightarrow \infty}|x|^{-\beta-1}\langle b(x), x\rangle<0$ нужно лишь для того, чтобы обеспечить интегрируемость $\exp \left(M|x|^{\beta}\right)$, и может быть заменено последней (однако его преимущество состоит в том, что оно выражено явно в терминах $A$ и $b)$.

Изложенные результаты обобщают результаты, полученные в [97], где применялась теория нелинейных уравнений (в частности, известные результаты С. Н. Бернштейна), что потребовало некоторых дополнительных предположений на $A$.

Используя полученные оценки, можно дать эффективно проверяемые условия принадлежности к $L^{p}(\mu)$ для логарифмического градиента $\nabla \varrho / \varrho$ меры $\mu$. В случае $p=2$ простые достаточные условия были получены [4], [5] и указаны выше. Первый общий результат для $p>2$ был установлен в [97] (специальный случай был рассмотрен в [100]). Условие из [22], приведенное в теореме ниже, усиливает этот результат, так как не требует дифференцируемость коэффициента сноса и предполагает более низкую регулярность диффузионного коэффициента (в [97] предполагается, что $a^{i j} \in C^{3}\left(\mathbb{R}^{d}\right)$ и $\left.b \in C^{2}\left(\mathbb{R}^{d}\right)\right)$. Это ослабление условий на коэффициенты стало возможным из-за того, что в отличие от [97] доказательство в [22] не использует методов теории нелинейных уравнений.

В следующей теореме и ее следствиях мы предположим, что $\mu$ - вероятностная мера на $\mathbb{R}^{d}$ с непрерывной положительной плотностью $\varrho$, удовлетворяющей эллиптическому уравнению (1.9.1) в смысле (1.9.2), т. е. мы имеем дело со случаем (I). В частности, корректно определены весовые классы Соболева $W^{p, 1}(\mu)$. 
Tеорема 1.9.3. Пусть $a^{i j} \in C^{0, \delta}\left(\mathbb{R}^{d}\right) \cap W_{\text {loc }}^{p_{0}, 1}\left(\mathbb{R}^{d}\right), \alpha \cdot \mathrm{I} \leqslant A \leqslant \gamma \cdot \mathrm{I}$, где $\alpha, \gamma, \delta>0, p_{0}>d$, причем $\lim _{r \rightarrow 0} \sup _{x}\left\|\partial_{x_{i}} a^{i j}\right\|_{L^{d}(U(x, r))}=0$ (nоследнее въполнено, если А липшищево). Пусть положительная непрерьвная функиия $\Phi \in W_{\text {loc }}^{1,1}\left(\mathbb{R}^{1}\right)$ возрастает на $[0,+\infty)$, причем $\Phi(N+1) \leqslant C \Phi(N)^{1+\varepsilon}$ при некотором $C, \varepsilon>0$, а функции $\Phi(|x|)$ u $\Phi^{\prime}(|x|)^{p_{1}}$ при некотором $p_{1}>d$ интегрируемы относительно меры $\mu$ на $\mathbb{R}^{d}$. Предположим также, что существуют такие числа $C_{0}>0, \theta>1, p>1 u \gamma \in[0,1 / d)$, что

$$
\begin{aligned}
& |b(x)| \leqslant C_{0} \Phi(|x|-\theta)^{\gamma}, \quad\left|\nabla a^{i j}(x)\right|^{d} \leqslant C_{0} \Phi(|x|), \\
& \sum_{N=1}^{\infty} N^{d-1} \Phi(N)^{-q}<\infty, \quad \text { где } q:=1-\gamma(p+\varepsilon d)>0 .
\end{aligned}
$$

Тогда $\ln \varrho \in W^{p, 1}(\mu)$.

СледСТвиЕ 1.9.4. Пусть $a^{i j} \in C^{0, \delta}\left(\mathbb{R}^{d}\right) \cap W_{\mathrm{loc}}^{p_{0}, 1}\left(\mathbb{R}^{d}\right), \alpha \cdot \mathrm{I} \leqslant A \leqslant \gamma \cdot \mathrm{I}$, где $\alpha, \gamma, \delta>0, p_{0}>d>1$, причем $\lim _{r \rightarrow 0} \sup _{x}\left\|\partial_{x_{i}} a^{i j}\right\|_{L^{d}(U(x, r))}=0$. Пусть дано $p>1$. Предположим, что для некоторых $M>0 u \beta>0$ бункиия $\exp \left(M|x|^{\beta}\right)$ интегрируема относительно меры $\mu$ и что

$$
|b(x)| \leqslant C_{0} \exp \left(\kappa|x|^{\beta}\right), \quad\left|\nabla a^{i j}(x)\right| \leqslant C_{0} \exp \left(\kappa|x|^{\beta}\right),
$$

где $0<\kappa d \max (p, d)<M$. Тогда $\ln \varrho \in W^{p, 1}(\mu)$. В частности, если для каждого $\kappa>0$ имеется такое число $C(\kappa)$, что

$$
|b(x)|+\left|\nabla a^{i j}(x)\right| \leqslant C(\kappa) \exp \left(\kappa|x|^{\beta}\right),
$$

mо $\ln \varrho \in W^{p, 1}(\mu)$ для всех $p \in[1,+\infty)$.

Взяв $\Phi(r)=r^{\beta}+1$, получаем следующий результат.

СледСТвиЕ 1.9.5. Пусть $a^{i j} \in C^{0, \delta}\left(\mathbb{R}^{d}\right) \cap W_{\text {loc }}^{p_{0}, 1}\left(\mathbb{R}^{d}\right), \alpha \cdot \mathrm{I} \leqslant A \leqslant \gamma \cdot \mathrm{I}$, где $\alpha, \gamma, \delta>0$ и $p_{0}>d>1$, причем $\lim _{r \rightarrow 0} \sup _{x}\left\|\partial_{x_{i}} a^{i j}\right\|_{L^{d}(U(x, r))}=0$. Пусть $p>1$. Предположим, что для некоторого $\beta>d$ бункиия $|x|^{\beta d}$ интегрируема относительно меры $\mu$ и что

$$
|b(x)| \leqslant C_{0}+C_{0}|x|^{\beta \gamma}, \quad\left|\nabla a^{i j}(x)\right| \leqslant C_{0}+C_{0}|x|^{\beta / d},
$$

где $0<\gamma<d^{-1}, \gamma<1-d \beta^{-1}$. Тогда $\ln \varrho$ входит в $W^{p, 1}(\mu)$ для каждого $p \in\left[1,(\beta-d) \beta^{-1} \gamma^{-1}\right)$.

Теперь мы рассмотрим нижние оценки без предположений о росте коэффициента сноса, но вместо этого использующие некоторую его интегрируемость относительно решения. До конца этого пункта мы будем предполагать, что матрица $A(x)=\left(a^{i j}(x)\right)_{1 \leqslant i, j \leqslant d}$ симметрична и удовлетворяет следующему условию:

(G1) для некоторого $p>d$ функиии $a^{i j}$ входят в класс $W_{\text {loc }}^{p, 1}\left(\mathbb{R}^{d}\right)$ и существуют такие числа $m, M>0$, что для всех $x, y \in \mathbb{R}^{d}$ мы имеем

$$
m|y|^{2} \leqslant \sum_{1 \leqslant i, j \leqslant d} a^{i j}(x) y_{i} y_{j} \leqslant M|y|^{2} .
$$


Если в дополнение к условию (G1) мы имеем $b^{i} \in L_{\mathrm{loc}}^{p}(\mu)$ (или $b^{i} \in L_{\mathrm{loc}}^{p}\left(\mathbb{R}^{d}\right)$ ), то $\mu$ задается непрерывной плотностью $\varrho \in W_{\mathrm{loc}}^{1, p}\left(\mathbb{R}^{d}\right)$, с которой и будем иметь дело. Уравнение (1.9.1) можно записать как равенство

$$
\partial_{x_{i}}\left(a^{i j} \partial_{x_{j}} \varrho\right)-\partial_{x_{i}}\left(b^{i} \varrho\right)=0,
$$

понимаемое в слабом смысле.

Метод получения нижних оценок, обсуждавшийся выше, неприменим в случае локально неограниченного $b$. В работе [101] было показано, что без всяких ограничений на рост $b$ можно получить оценки вида

$$
\varrho(x) \geqslant \exp \left(-f\left(c_{1}|x|+c_{2}\right)\right)
$$

где $c_{1}, c_{2}$ - некоторые положительные числа и функция $f \in C^{2}([0, \infty))$ удовлетворяет условиям

(H1) $f(z)>0, f^{\prime}(z)>0, f^{\prime \prime}(z)>0$ nрu $z>0$;

(Н2) функция $e^{-f(z)}$ выпукла (m.е. $\left.\left(e^{-f}\right)^{\prime \prime} \geqslant 0\right)$ на множестве $z>z_{0}$ для некоторого $z_{0} \geqslant 0$ и убывает $\kappa 0$ при $z \rightarrow+\infty$.

Для получения оценки (1.9.4) достаточно в дополнение к (G1) потребовать выполнение следующего условия:

(G2) $|b| \exp (\psi(|b|)) \in L^{p}(\mu)$, где $p>\min \{2, d\}$ и $\psi$ - неотрицательная строго возрастающая непрерывная функиия, отображающая $[0, \infty)$ на $[0, \infty)$, причем для некоторого $N>0$ и всех $z>0$ выполнено неравенство

(Н3) $\psi^{-1}(z) \leqslant N f^{\prime}\left(f^{-1}(z)\right)$.

Дадим несколько типичных примеров функций $f$ и $\psi$. Пусть $\delta>0$ - заданное число. Если $f(z)=e^{z}$, то можно взять $\psi(z)=\delta \cdot z$. В этом случае получим оценку

$$
\varrho(x) \geqslant \exp \left(-\tilde{c}_{2} \exp \left(\tilde{c}_{1}|x|\right)\right) .
$$

Если $f(z)=z^{r /(r-1)}$ и $r>1$, то подходит $\psi(z)=\delta \cdot z^{r}$. Тогда

$$
\varrho(x) \geqslant \tilde{c}_{2} \exp \left(-\tilde{c}_{1}|x|^{r /(r-1)}\right) .
$$

В случае, когда $d=1, A=1$ и $b=\varrho^{\prime} / \varrho$, такие оценки были получены в [102] (и распространены на случай $d>1$ в [103] при том же предположении, что $A=\mathrm{I}$ и $b=\nabla \varrho / \varrho)$. Из (1.9.4) следует, что плотность решения не имеет нулей при условии, более слабом, чем экспоненциальная интегрируемость $|b|$ (достаточность последнего условия была доказана в [104]). Например, если положить $f(z)=e^{e^{z}}$ и $\psi(z)=\delta z /|\ln z|^{\kappa}$ при $z>2$ и $0<\kappa<1$, то получим условие, которое достаточно для положительности, но слабее экспоненциальной интегрируемости $|b|$. Если $d=1, A=1$ и $b=\varrho^{\prime} / \varrho$, то это новое достаточное условие положительности близко к полученному в [105], а последнее в некотором смысле неулучшаемо.

Положим $V=e^{f} / f^{\prime}$.

Так как $\left(e^{-f}\right)^{\prime \prime}=\left[\left(f^{\prime}\right)^{2}-f^{\prime \prime}\right] e^{-f} \geqslant 0$ на $\left[z_{0},+\infty\right)$, то

$$
V^{\prime}=\left[\left(f^{\prime}\right)^{2}-f^{\prime \prime}\right] e^{-f}\left(f^{\prime}\right)^{-2} \geqslant 0 \quad \text { на }\left[z_{0},+\infty\right) .
$$

Кроме того, $V$ возрастает к $+\infty$, ибо функция $1 / V=f^{\prime} e^{-f}$ не может быть отделена от нуля на $[0,+\infty)$. Из условий $(\mathrm{H} 1)$ и $(\mathrm{H} 3)$ следует, что $f^{\prime}(y) \rightarrow+\infty$ 
при $y \rightarrow+\infty$. Следовательно, найдется такое $y_{0}>\max \left\{z_{0}, 1\right\}$, что $f^{\prime}(y) \geqslant 1$ и $V(y) \geqslant e^{\psi(0)}$ при $y>y_{0}$. Пусть $\tau_{0}:=\exp \left(-f\left(\ln y_{0}\right)\right)$. Тогда $0<\tau_{0}<1$.

Зафиксируем куб $Q$ с единичным ребром. Положим

$$
\Lambda:=\min \left\{\tau_{0}\left(2\|\varrho\|_{L^{\infty}\left(\mathbb{R}^{d}\right)}\right)^{-1}, 1\right\} .
$$

Теорема 1.9.6. Пусть $\mu=\varrho d x$ - решение уравнения (1.9.1), где коэфбициенты $a^{i j}, b^{i}$ удовлетворяют условиям (G1), (G2), причем выполнены условия (H1), (Н2) и (Н3). Тогда существуют такие числа $C>0 u \alpha>0$, ито для каждого измеримого множества $E \subset Q$ имеем

$$
\sup _{x \in Q} \exp \left(f^{-1}(|\ln (\Lambda \varrho)|)\right) \leqslant C\left(\int_{E} \exp (-\alpha f(|\ln (\Lambda \varrho)|)) d x\right)^{-1 / \alpha},
$$

где числа С и а зависят только от следующих величин:

$$
p, N, N_{1}, \tau_{0}, m, M, d,\|\varrho\|_{L^{\infty}\left(\mathbb{R}^{d}\right)}, \int_{\mathbb{R}^{d}}|b|^{p} e^{p \psi(|b|)} \varrho d x .
$$

Теорема 1.9.7. Пусть $\mu=\varrho d x$ - решение уравнения (1.9.1), где коэфбициенты $a^{i j}, b^{i}$ удовлетворяют условиям (G1), (G2), причем выполнены условия (H1), (Н2) и (Н3). Тогда существуют такие числа $c_{1}>0$ и $c_{2}>0$, что

$$
\varrho(x) \geqslant e^{-f\left(c_{1}|x|+c_{2}\right)}, \quad x \in \mathbb{R}^{d} .
$$

Этот результат дает нижние оценки плотности стационарной меры диффузионного процесса с диффузионным коэффициентом $\sqrt{2 A}$ и сносом $b$.

ПримеР 1.9.8. Пусть выполнено условие (G1) и дано число $r>1$.

(i) Чтобы получить оценку

$$
\varrho(x) \geqslant \tilde{c}_{2} \exp \left(-\tilde{c}_{1}|x|^{r /(r-1)}\right),
$$

достаточно иметь $\exp \left(\delta|b|^{r}\right) \in L^{1}(\mu)$ при некотором $\delta>0$.

В самом деле, функция $\psi(z)=\delta z^{r} /(2 p)$ удовлетворяет условию (Н3) для

$$
f(z)=z^{r /(r-1)} .
$$

Существует такое $C(\delta)>0$, что $|z| \leqslant C(\delta) \exp \left(\delta|z|^{r} / 2\right)$. Тогда

$$
\left(|b| \exp \left(\frac{\delta|b|^{r}}{2 p}\right)\right)^{p} \leqslant C(\delta)^{p} \exp \left(\delta|b|^{r}\right)
$$

и потому $|b| \exp \left(\delta|b|^{r} /(2 p)\right) \in L^{p}(\mu)$, т. е. выполнено условие (G2).

(ii) Чтобы получить оценку

$$
\varrho(x) \geqslant \exp \left(-\tilde{c}_{2} \exp \left(\tilde{c}_{1}|x|\right)\right),
$$

достаточно иметь $\exp (\delta|b|) \in L^{1}(\mu)$ при некотором $\delta>0$.

В самом деле, при $0<\delta_{1}<\delta$ функции $\psi(z)=\delta_{1} z$ и $f(z)=e^{z}$ удовлетворяют (Н3) с $N=1 / \delta_{1}$, причем выполнено (G2). 
ПримеР 1.9.9. Пусть $\mu=\varrho d x$ - вероятностная мера, $\varrho \in W_{\mathrm{loc}}^{1,1}\left(\mathbb{R}^{d}\right)$. Тогда $\mu$ очевидным образом удовлетворяет уравнению (1.9.1) с $A=\mathrm{I}$ и $b=\nabla \varrho / \varrho$, где $b(x):=0$, если $\varrho(x)=0$. Следовательно, чтобы получить оценку $(1.9 .5)$, достаточно иметь $\exp \left(\delta|\nabla \varrho / \varrho|^{r}\right) \in L^{1}(\mu)$ при некотором $\delta>0$, а оценка (1.9.6) следует из включения $\exp (\delta|\nabla \varrho / \varrho|) \in L^{1}(\mu)$ при некотором $\delta>0$.

Для $d=1$ утверждение этого примера было получено в [102] (где в случае $r=1$ формулировка содержит небольшую неточность: $\tilde{c}_{1}$ заменено на 1 ; функция $\varrho(x)=\exp (-\exp (2|x|))$ показывает, что от $\tilde{c}_{1}$ избавиться нельзя). Для $d>1$ и $r=1$ аналогичное утверждение дано в [26; задача 6.8.4]; в [103] рассмотрен случай $r>1$. Однако методы работ [102] и [103] существенным образом используют тот факт, что $b$ есть $\nabla \varrho / \varrho$.

\section{2. Параболические уравнения для мер}

2.1. Априорные оценки. В этом разделе рассматриваются параболические уравнения вида

$$
L^{*} \mu=0
$$

для борелевских мер $\mu$ на $\mathbb{R}^{d} \times(0,1)$. Здесь $L$ - параболический оператор второго порядка

$$
L u(x, t):=\frac{\partial u(x, t)}{\partial t}+a^{i j}(x, t) \partial_{x_{i}} \partial_{x_{j}} u(x, t)+b^{i}(x, t) \partial_{x_{i}} u(x, t),
$$

где $A(x, t):=\left(a^{i j}(x, t)\right)_{i, j \leqslant d}$ - неотрицательная симметричная матрица размерности $d$ и $b(x, t):=\left(b^{i}(x, t)\right)_{i \leqslant d}-$ вектор в $\mathbb{R}^{d}$, а интерпретация нашего уравнения состоит в следующем. Будем говорить, что семейство радоновских мер $\mu=\left(\mu_{t}\right)_{t \in(0,1)}$ на $\mathbb{R}^{d}$ удовлетворяет слабому параболическому уравнению (2.1.1), если функции $a^{i j}$ и $b^{i}$ интегрируемы на каждом компактном множестве в $\mathbb{R}^{d} \times(0,1)$ относительно меры $\mu(d x d t):=\mu_{t}(d x) d t$ на $\mathbb{R}^{d} \times(0,1)$ (ниже мы обозначаем меру $\mu_{t}(d x) d t$ также через $\mu$ ) и для каждой функции $u \in C_{0}^{\infty}\left(\mathbb{R}^{d} \times(0,1)\right)$ мы имеем

$$
\int_{0}^{1} \int_{\mathbb{R}^{d}} L u(x, t) \mu_{t}(d x) d t=0 .
$$

Таким образом, интерпретация - такая же, как и в эллиптическом случае. Для операторов дивергентного вида

$$
\mathscr{L} u(x, t):=\frac{\partial u(x, t)}{\partial t}+\partial_{x_{i}}\left(a^{i j}(x, t) \partial_{x_{j}} u(x, t)\right)+b^{i}(x, t) \partial_{x_{i}} u(x, t)
$$

уравнение

$$
\mathscr{L}^{*} \mu=0
$$

определяется аналогично (здесь, как и в п. 1.9, возможны два случая).

Будем говорить, что $\mu$ удовлетворяет начальному условию $\mu_{0}:=\nu$ при $t=0$, если $\nu$ - мера на $\mathbb{R}^{d}$ и

$$
\lim _{t \rightarrow 0} \int_{\mathbb{R}^{d}} \zeta(x) \mu_{t}(d x)=\int_{\mathbb{R}^{d}} \zeta(x) \nu(d x)
$$

для всех $\zeta \in C_{0}^{\infty}\left(\mathbb{R}^{d}\right)$. В этом случае будем писать $\mu=\left(\mu_{t}\right)_{t \in[0,1)}$. 
Такие же определения вводятся в случае, когда вместо $\mathbb{R}^{d}$ берется открытое множество $\Omega \subset \mathbb{R}^{d}$ или открытое множество в римановом многообразии. В частности, в (2.1.2) берутся $u \in C_{0}^{\infty}(\Omega \times(0,1))$ и в (2.1.3) берется $\zeta \in C_{0}^{\infty}(\Omega)$.

Уравнение (2.1.1) выполнено для переходных вероятностей диффузионного процесса с диффузионной матрицей $\sqrt{2 A}$ и сносом $b$, при условии, что такая диффузия существует и коэффициенты $A$ и $b$ удовлетворяют некоторым условиям (см., например, [106; гл. 2, 3]; указанные там условия могут быть еще ослаблены на основе недавнего прогресса в теории уравнений с коэффициентами класса VMO, см., например, [38]-[40]). Этот диффузионный процесс может существовать в различных смыслах, например, как подходящее решение стохастического дифференциального уравнения

$$
d \xi_{t}=\sigma\left(\xi_{t}\right) d w_{t}+b\left(\xi_{t}\right) d t, \quad A=\frac{1}{2} \sigma \sigma^{*} .
$$

Однако (2.1.1) может рассматриваться независимо от всяких вероятностных предположений. Более того, изучение этого уравнения в чисто аналитической постановке может быть полезно для построения ассоциированной диффузии (см. [50], [107]).

Стоит отметить, что (2.1.2) можно записать как

$$
\frac{\partial \mu}{\partial t}=\partial_{x_{i}} \partial_{x_{j}}\left(a^{i j} \mu\right)-\partial_{x_{i}}\left(b^{i} \mu\right)
$$

в смысле теории обобщенных функций на $(0,1) \times \mathbb{R}^{d}$. Оказывается, что при довольно мягких ограничениях на $A$ и $b$, указанных ниже, всякое решение $\mu$ имеет плотность $\varrho$, обладающую некоторой соболевской регулярностью по $x$, так что это равенство можно далее переписать в терминах классических слабых решений.

Для функции $u$ на $\mathbb{R}^{d} \times(0,1)$ положим

$$
\partial_{t} u(x, t):=\frac{\partial u(x, t)}{\partial t}, \quad \nabla u(x, t)=\left(\partial_{x_{1}} u(x, t), \ldots, \partial_{x_{d}} u(x, t)\right) .
$$

Этот пункт посвящен некоторым априорным оценкам решений; доказательства можно найти в [19].

Лемма 2.1.1. Если $\mu=\left(\mu_{t}\right)_{t \in[0,1)}$ удовлетворяет (2.1.2) u (2.1.3), то для каждого $\zeta \in C_{0}^{\infty}\left(\mathbb{R}^{d}\right)$ для почти всех $t \in[0,1)$ имеем

$$
\int_{\mathbb{R}^{d}} \zeta(x) \mu_{t}(d x)-\lim _{\varepsilon \rightarrow 0} \int_{\varepsilon}^{t} \int_{\mathbb{R}^{d}} L \zeta(x, s) \mu_{s}(d x) d s=\int_{\mathbb{R}^{d}} \zeta(x) \nu(d x) .
$$

Если для каждого $\zeta \in C_{0}^{\infty}\left(\mathbb{R}^{d}\right)$ функция $t \mapsto \int_{\mathbb{R}^{d}} \zeta(x) \mu_{t}(d x)$ непрерьвна на $[0,1)$, то (2.1.5) выполнено для всех $t \in[0,1)$ и равносильно (2.1.1) u (2.1.3). Это же верно в случае, когда наше уравнение рассматривается на открытом множестве.

Заметим, что если $\mu_{t}$ - вероятностные меры и есть такая $\mu$-интегрируемая функция $\Theta$, что $L \zeta(x, s) \leqslant \Theta(x, s) \quad \mu$-п. в., то функция

$$
h: s \mapsto \int_{\mathbb{R}^{d}} L \zeta(x, s) \mu_{s}(d x)
$$


интегрируема на $[0, t]$ (так что предел интегралов по $[\varepsilon, t]$ равен интегралу по $[0, t])$. В самом деле, в этом случае функция $h$, которая совпадает с производной непрерывной версии функции

$$
f(s):=\int_{\mathbb{R}^{d}} \zeta(x) \mu_{s}(d x)
$$

на $(0,1)$, мажорируется интегрируемой функцией $s \mapsto \int_{\mathbb{R}^{d}} \Theta(x, s) \mu_{s}(d x)$. Поскольку $f$ ограничена, это означает, что непрерывная версия $f$ имеет конечный предел в 0 и абсолютно непрерывна на $[0,1]$. Конечно, все это заведомо верно, если функции $a^{i j}$ и $b^{i}$ являются $\mu$-интегрируемыми на каждом множестве $[0,1] \times B$, где $B-$ шар в $\mathbb{R}^{d}$.

Стоит отметить, что одна из причин, почему мы требуем ниже, чтобы все (а не просто почти все) меры $\mu_{t}$ были вероятностными, состоит в том, что это имеет место в случае, когда мы имеем дело с переходными вероятностями. С аналитической точки зрения, это, конечно, несущественно. Другая причина это то, что, как мы увидим ниже, такое предположение упрощает некоторые технические моменты.

Следующая лемма из [19] является непосредственным обобщением [18; лемма 2.2], где $M=0$ и $\Theta$ - постоянная.

Лемма 2.1.2. Пусть $\mu=\left(\mu_{t}\right)_{t \in[0,1)}$ - семейство вероятностных мер на $\mathbb{R}^{d}$, удовлетворяющее (2.1.1) и (2.1.3), где $\nu$ - вероятностная мера на $\mathbb{R}^{d}$. Предположим, что существуют такие н-интегрируемая функция $\Theta$, неотрицательная функиия $\Psi \in C^{2}\left(\mathbb{R}^{d}\right)$ и число $M$, что $\Psi \in L^{1}(\nu), \lim _{|x| \rightarrow \infty} \Psi(x)=+\infty$ и выполнена оценка

$$
L \Psi(x, t) \leqslant \Theta(x, t)+M \Psi(x) \quad \mu_{t} d t-n . \text {. } .
$$

Тогда для почти всех $t \in[0,1)$ имеем

$$
\begin{aligned}
\int_{\mathbb{R}^{d}} \Psi d \mu_{t} \leqslant & \int_{\mathbb{R}^{d}} \Psi d \nu+\int_{0}^{t} \int_{\mathbb{R}^{d}} \Theta d \mu_{s} d s \\
& +M \exp (M t) \int_{0}^{t} \exp (-M s)\left[\int_{\mathbb{R}^{d}} \Psi d \nu+\int_{0}^{s} \int_{\mathbb{R}^{d}} \Theta d \mu_{r} d r\right] d s \\
\leqslant & \left(M e^{M}+1\right)\left[\|\Psi\|_{L^{1}(\nu)}+\|\Theta\|_{L^{1}(\mu)}\right]
\end{aligned}
$$

Если $M=0$ и $\Theta=K-$ постояннал, то для почти всех $t \in[0,1)$ имеем

$$
\int_{\mathbb{R}^{d}} \Psi(x) \mu_{t}(d x) \leqslant t K+\int_{\mathbb{R}^{d}} \Psi(x) \nu(d x) .
$$

Более того, если функиии

$$
t \mapsto \int \zeta(x) \mu_{t}(d x), \quad \text { əде } \zeta \in C_{0}^{\infty}\left(\mathbb{R}^{d}\right),
$$

непрерывны на $[0,1)$, то (2.1.7), а в случае $M=0$ также $(2.1 .8)$, верно для всех $t \in[0,1)$. 
Если (2.1.1) и (2.1.3) выполнены на открытом множестве $\{\Psi<c\}$, то вернъ такие же утверждения $c\{\Psi<c\}$ вместо $\mathbb{R}^{d}$. Наконеи, предположение, что каждая $\mu_{t}$ - вероятностная мера, можно заменить следующим: $\mu_{t} \geqslant 0$ $u\left\|\mu_{t}\right\| \leqslant\|\nu\|$.

СЛЕДСТВИЕ 2.1.3. Пусть $\mu=\left(\mu_{t}\right)_{t \in[0,1)}$ - семейство вероятностных мер на $\mathbb{R}^{d}$, удовлетворяющее (2.1.1) и (2.1.3), где $\nu$ - вероятностная мера на $\mathbb{R}^{d}$. Пусть $\Psi \in C^{2}\left(\mathbb{R}^{d}\right)$ - такая неотрицательная функция, что

$$
\lim _{|x| \rightarrow \infty} \Psi(x)=+\infty \quad u \quad L \Psi(x, t) \leqslant C+M \Psi(x) \quad \mu_{t} d t-n . \text { в. }
$$

где $C \geqslant 0$ и $M \geqslant 0$ - постоянные. Тогда найдется такая неотрицательная функиия $\Psi_{0} \in C^{2}\left(\mathbb{R}^{d}\right)$, ито

$$
\Psi_{0} \in L^{1}(\nu), \quad \lim _{|x| \rightarrow \infty} \Psi_{0}(x)=+\infty \quad \text { u } L \Psi_{0}(x, t) \leqslant C+M \quad \mu_{t} d t-n .8 .
$$

Более того, если $\mathfrak{M}$ - равномерно плотное семейство вероятностных мер на $\mathbb{R}^{d}$ и для каждой мерь $\nu \in \mathfrak{M}$ существует решение $\mu^{\nu}=\left(\mu_{t}^{\nu}\right)_{t \in[0,1)}$ задачи $L_{\nu}^{*} \mu^{\nu}=0, \mu_{0}^{\nu}=\nu$ в смысле (2.1.1), (2.1.3), где каждый оператор $L_{\nu}$ удовлетворяет тем же условиям, что $L$, и для некоторой неотрицательной компактной функции $\Psi \in C^{2}\left(\mathbb{R}^{d}\right)$ мы имеем

$$
L_{\nu} \Psi(x, t) \leqslant C+M \Psi(x) \quad \mu_{t}^{\nu} d t-n . \text { в. }
$$

то найдется такая функиия $\Psi_{0}$ с указанными выще свойствами, что

$$
\sup _{\nu \in \mathfrak{M}} \operatorname{ess} \sup _{t \in[0,1)} \int_{\mathbb{R}^{d}} \Psi_{0} d \mu_{t}^{\nu} \leqslant C+M+\sup _{\nu \in \mathfrak{M}} \int_{\mathbb{R}^{d}} \Psi_{0} d \nu<\infty .
$$

Если же функиии $t \mapsto \int \zeta d \mu_{t}$, где $\zeta \in C_{0}^{\infty}\left(\mathbb{R}^{d}\right)$, непрерывны на $[0,1]$, то ess sup можно заменить на sup. Эти же утверждения верны в случае, когда вместо $\mathbb{R}^{d}$ берется $\{\Psi<c\}$.

Рассмотрим примеры, как (2.1.6) можно проверить в терминах коэффициентов $L$.

ПРимеР 2.1.4. (i) Предположим, что

$$
\left|a^{i j}(x, t)\right| \leqslant c_{1}+c_{2}|x|^{2}, \quad\langle b(x, t), x\rangle \leqslant c_{3}+c_{4}|x|^{2}
$$

для некоторых постоянных $c_{i}$. Тогда, полагая $\Psi(x):=|x|^{2 k}, k>0$, получаем $L \Psi \leqslant C+C \Psi$ для достаточно большого числа $C>0$. Следовательно, если решение $\mu$ существует и $|x|^{2 k} \in L^{1}\left(\mu_{0}\right)$, то для почти всех $t$ имеем

$$
\int_{\mathbb{R}^{d}}|x|^{2 k} \mu_{t}(d x) \leqslant e^{C} \int_{\mathbb{R}^{d}}|x|^{2 k} \mu_{0}(d x)+C e^{C} .
$$

(ii) Предположим, что

$$
\left|a^{i j}(x, t)\right| \leqslant c_{1}+c_{2} \ln \left(|x|^{2}+1\right), \quad\langle b(x, t), x\rangle \leqslant c_{3}+c_{4}|x|^{2}+c_{5}|x|^{2} \ln \left(|x|^{2}+1\right)
$$


для некоторых постоянных $c_{i}$. Тогда, полагая $\Psi(x):=\ln \left(|x|^{2}+1\right)$, находим

$$
\begin{aligned}
\partial_{x_{i}} \Psi(x) & =2 x_{i}\left(|x|^{2}+1\right)^{-1}, \\
\partial_{x_{j}} \partial_{x_{i}} \Psi(x) & =2 \delta_{i j}-4 x_{i} x_{j}\left(|x|^{2}+1\right)^{-2},
\end{aligned}
$$

что дает

$$
\begin{aligned}
L \Psi(x, t) & =2 \operatorname{trace} A(x, t)-4\left(|x|^{2}+1\right)^{-2}\langle A(x, t) x, x\rangle+2\left(|x|^{2}+1\right)^{-1}\langle b(x, t), x\rangle \\
& \leqslant C+C \Psi(x)
\end{aligned}
$$

для достаточно большого числа $C>0$. Следовательно, если решение $\mu$ существует и $\ln \left(|x|^{2}+1\right) \in L^{1}\left(\mu_{0}\right)$, то для почти всех $t$ имеем

$$
\int_{\mathbb{R}^{d}} \ln \left(|x|^{2}+1\right) \mu_{t}(d x) \leqslant e^{C} \int_{\mathbb{R}^{d}} \ln \left(|x|^{2}+1\right) \mu_{0}(d x)+C e^{C} .
$$

Более того, полагая $\Psi(x)=\left|\ln \left(|x|^{2}+1\right)\right|^{2}$, получаем также $L \Psi \leqslant C+C \Psi$, значит,

$$
\int_{\mathbb{R}^{d}}\left|\ln \left(|x|^{2}+1\right)\right|^{2} \mu_{t}(d x) \leqslant e^{C} \int_{\mathbb{R}^{d}}\left|\ln \left(|x|^{2}+1\right)\right|^{2} \mu_{0}(d x)+C e^{C} .
$$

(iii) Предположим, что

$$
\langle A(x, t) x, x\rangle \leqslant \gamma_{1}+\alpha|x|^{2 \beta}, \quad\langle b(x, t), x\rangle \leqslant \gamma_{2}-(2 \alpha c k+\varepsilon)|x|^{2 k+2 \beta-2}
$$

с некоторыми положительными постоянными $\gamma_{1}, \gamma_{2}, \alpha, \beta, c, k, \varepsilon$. Возьмем функцию $\Psi(x)=\exp \left(c|x|^{2 k}\right)$. Тогда

$$
\begin{aligned}
L \Psi(x, t)= & 2 c k \text { trace } A(x, t)|x|^{2 k-2} \Psi(x)+4 c k(k-1)\langle A(x, t) x, x\rangle|x|^{2 k-4} \Psi(x) \\
& +(2 c k)^{2}\langle A(x, t) x, x\rangle|x|^{4 k-4} \Psi(x)+2 c k|x|^{2 k-2} \Psi(x)\langle b(x, t), x\rangle \\
\leqslant & c_{0}-\varepsilon|x|^{2 k+2 \beta-2} \Psi(x)
\end{aligned}
$$

с некоторой постоянной $c_{0}$. Значит, если $\beta \geqslant 1$ и $\Psi \in L^{1}\left(\mu_{0}\right)$, то

$$
\underset{t \in[0,1)}{\operatorname{ess} \sup } \int_{\mathbb{R}^{d}} \exp \left(c|x|^{2 k}\right) \mu_{t}(d x)<\infty .
$$

Введем следующие условия на $A, b, p \in[1,+\infty)$ и ограниченное открытое множество $B \subset \mathbb{R}^{d}$ :

(CP1) существуют два числа $M_{1}=M_{1}(B)>0$ и $M_{2}=M_{2}(B)$ такие, что для всех $i, j$ мы имеем

$$
A(x, t) \geqslant M_{1} \cdot \mathrm{I} \quad \forall(x, t) \in B \times(0,1), \quad \sup _{t \in(0,1)}\left\|a^{i j}(\cdot, t)\right\|_{W^{p, 1}(B)} \leqslant M_{2} ;
$$

(CP2) существует такое $M_{3}=M_{3}(B)$, что для всех $і$ мы имеем

$$
\sup _{t \in(0,1)}\left\|b^{i}(\cdot, t)\right\|_{L^{p}(B)} \leqslant M_{3}
$$


Из (CP1) и теоремы вложения Соболева вытекает, что если $p>d$, то каждая функция $a^{i j}$ имеет совместно измеримую версию, для которой все функции $x \mapsto a^{i j}(x, t), t \in(0,1)$, гёльдеровы порядка $1-d / p$ и ограничены на $B$ равномерно по $t$ (их гёльдеровы и sup-нормы на $B$ оцениваются через постоянные, зависящие от $p, d, B$ и $\left.M_{2}\right)$. Ниже используются те же обозначения $a^{i j}$ для этих специальных версий.

Главная теорема существования, приведенная ниже, основана на следующей лемме.

Положим

$$
b_{0}:=A^{-1 / 2}(b-\Gamma), \quad \Gamma:=\left(\Gamma^{1}, \ldots, \Gamma^{d}\right), \quad \Gamma^{j}=\partial_{x_{i}} a^{i j} .
$$

Лемма 2.1.5. Пусть $\Omega$ - ограниченное открытое множество в $\mathbb{R}^{d}$ с границей класса $C^{1}$ и обгемом $|\Omega|$, а функиии $a^{i j}$ и $b^{i}$ равномерно ограничены на $\bar{\Omega} \times(0,1)$ вместе с их первыми и вторыми производными по второму аргументу. Предположим, что $\mu=\varrho(x, t) d x d t$, где каждая $\varrho(\cdot, t), t>0$, является неотрицательной функиией на $\Omega$ с ограниченными на $\Omega \times(0,1)$ вторыми производными по $x$, причем

$$
\frac{\partial \varrho(x, t)}{\partial t}=\partial_{x_{i}} \partial_{x_{j}}\left(a^{i j} \varrho\right)-\partial_{x_{i}}\left(b^{i} \varrho\right)
$$

в множестве $\Omega \times(0,1)$. Предположим также, что функиии $\varrho$ и $\partial_{x_{i}} \varrho$ непрерывны на $\bar{\Omega} \times[0,1], \varrho(x, 0)=\varrho_{0}(x)$, где $\varrho_{0} \in C_{0}^{1}(\Omega), u$

$$
\left\langle A \nabla \varrho(x, t)+[\Gamma(x, t)-b(x, t)] \varrho(x, t), \mathrm{n}_{\partial \Omega}(x)\right\rangle=0, \quad(x, t) \in \partial \Omega \times(0,1),
$$

где $\mathrm{n}_{\partial \Omega}$ - внешняя единичная нормаль на $\partial \Omega$. Тогда выполнены следующие неравенства:

$$
\begin{aligned}
& \int_{\Omega} \varrho(x, t)^{2} d x+\int_{0}^{t} \int_{\Omega}|\sqrt{A} \nabla \varrho(x, s)|^{2} d x d s \\
& \leqslant \int_{\Omega} \varrho(0, x)^{2} d x+\int_{0}^{t} \int_{\Omega}\left|b_{0}(x, s)\right|^{2} \varrho(x, s) d x d s, \\
& \int_{\Omega} \varrho(x, t)^{2} d x+\int_{0}^{t} \int_{\Omega}|\sqrt{A} \nabla \varrho(x, s)|^{2} d x d s \\
& \leqslant e^{t / 2} \int_{\Omega} \varrho(x, 0)^{2} d x+\frac{1}{2} e^{t / 2}\left\|b_{0}\right\|_{L^{4}(\Omega \times[0,1])}^{4} .
\end{aligned}
$$

Если $A(x, t) \geqslant \alpha \cdot$ I для некоторого числа $\alpha>0$ икаждая $\varrho(\cdot, t), t \in[0,1]$, является вероятностной плотностью, или, более общим образом, мы имеем $0<\mu_{t}(\Omega) \leqslant \mu_{0}(\Omega)$, mo

$$
\begin{aligned}
& \int_{0}^{1} \int_{\Omega} \frac{|\sqrt{A} \nabla \varrho(x, t)|^{2}}{\varrho(x, t)} d x d t \\
& \quad \leqslant 2 \int_{0}^{1} \int_{\Omega}\left|b_{0}(x, t)\right|^{2} \varrho(x, t) d x d t+2 \int_{\Omega} \varrho_{0}(x) \ln \varrho_{0}(x) d x+2|\Omega| .
\end{aligned}
$$


СлЕДСТвИЕ 2.1.6. В ситуащии леммы выше существует такая постоянная $C(\Omega)$, что

$$
\begin{aligned}
& \int_{0}^{1}\left(\int_{\Omega}|\varrho(x, t)|^{d /(d-2)} d x\right)^{(d-2) / d} d t \leqslant \frac{C(\Omega)}{\alpha}\left(\int_{0}^{1} \int_{\Omega}\left|b_{0}(x, t)\right|^{2} \varrho(x, t) d x d t\right. \\
& \left.\quad+\int_{\Omega} \varrho_{0}(x) \ln \varrho_{0}(x) d x+|\Omega|\right)+2|\Omega|^{(2 d-2) / d}
\end{aligned}
$$

если $d>2$. В случае $d \leqslant 2$ похожая оценка верна для всякого $r<\infty$ вмеcmo $d /(d-2)$.

Наконеи, для каждого $p>d$ имеется такая постоянная $C(\Omega, p)$, что

$$
\begin{aligned}
& \int_{0}^{1} \int_{\Omega}|\sqrt{A} \nabla \varrho(x, t)|^{2} \varrho(x, t)^{-1} d x d t \\
& \quad \leqslant C(\Omega, p) \sup _{t \in(0,1)}\left(\int_{\Omega}\left|b_{0}(x, t)\right|^{p} d x\right)^{2 d /(p-d)}+2 \int_{\Omega} \varrho_{0}(x) \ln \varrho_{0}(x) d x+C(\Omega, p) .
\end{aligned}
$$

СледСтвиЕ 2.1.7. Предположим, что в лемме 2.1.5 существуют такие неотрицательная функиия $\Psi \in C^{2}\left(\mathbb{R}^{d}\right)$ и постоянная $M \geqslant 0$, что мы имеем $\Omega=\left\{x \in \mathbb{R}^{d}: \Psi(x)<c\right\} u$

$$
L \Psi(x, t) \leqslant M+M \Psi(x) \quad u \quad\left|b_{0}(x, t)\right|^{2} \leqslant \Psi(x) .
$$

Тогда

$$
\begin{aligned}
& \int_{\Omega} \varrho(x, t)^{2} d x+\int_{0}^{1} \int_{\Omega}|\sqrt{A} \nabla \varrho(x, t)|^{2} d x d t \\
& \leqslant 2\left(M e^{M}+1\right) \int_{\Omega} \Psi(x) \varrho_{0}(x) d x+2 M\left(M e^{M}+1\right)+\int_{\Omega} \varrho_{0}(x)^{2} d x \\
& \int_{0}^{1} \int_{\Omega}|\sqrt{A} \nabla \varrho(x, t)|^{2} \varrho(x, t)^{-1} d x d t \leqslant 2\left(M e^{M}+1\right) \int_{\Omega} \Psi(x) \varrho_{0}(x) d x \\
& \quad+2 M\left(M e^{M}+1\right)+2 \int_{\Omega} \varrho_{0}(x) \ln \varrho_{0}(x) d x+2|\Omega|
\end{aligned}
$$

В частности, это верно для $\Psi(x)=|x|^{2 k}$ при $k \geqslant 1$ при условии, что $\operatorname{trace} A(x, t) \leqslant C+C|x|^{2}, \quad\left|b_{0}(x, t)\right|^{2} \leqslant C+C|x|^{2 k}, \quad\langle b(x, t), x\rangle \leqslant C+C|x|^{2}$.

2.2. Локальная регулярность. Пусть $J$ - интервал и $U$ - открытое множество в $\mathbb{R}^{d}$. Обозначим через $\mathbb{H}^{p, s}(U, J)$ пространство всех измеримых функций $u$ на $U \times J$ таких, что $u(\cdot, t) \in H^{p, s}(U)$ и конечна норма

$$
\|u\|_{\mathbb{H}^{p, s}(U, J)}=\left(\int_{J}\|u(\cdot, t)\|_{H^{p, s}(U)}^{p} d t\right)^{1 / p} .
$$

Пространство $\mathbb{H}_{0}^{p, s}(U, J)$ определяется аналогично, но берется $H_{0}^{p, s}(U)$ вместо $H^{p, s}(U)$, а через $\mathbb{H}^{p^{\prime},-s}(U, J)$ обозначается его сопряженное. В связи с параболическими уравнениями полезно ввести также следующие пространства. 
Пусть $\mathscr{H}^{p, 1}(U, J)$ - пространство всех функций $u \in \mathbb{H}^{p, 1}(U, J)$, для которых $\partial_{t} u \in \mathbb{H}^{p,-1}(U, J)$ и конечна норма

$$
\|u\|_{\mathscr{H}^{p, 1}(U, J)}=\left\|\partial_{t} u\right\|_{\mathbb{H}^{p,-1}(U, J)}+\|u\|_{\mathbb{H}^{p, 1}(U, J)} .
$$

Обозначим через $B_{R}$ открытый шар радиуса $R>0$ с центром в некоторой точке из $\mathbb{R}^{d}$ (в случае, когда центр важен, мы используем символ $B(a, R)$ ). Через $H_{0}^{p, 2 ; 1}\left(B_{R},[0, T]\right)$ обозначим замыкание пространства гладких функций $u$ на цилиндре $B_{R, T}:=B_{R} \times[0, T]$, которые равны нулю на $\partial B_{R} \times[0, T] \cup B_{R} \times\{0\}$ (т. е. имеют нулевой предел на этой части границы), относительно нормы

$$
\begin{aligned}
\|u\|_{H_{0}^{p, 2 ; 1}\left(B_{R},[0, T]\right)}=\| & u\left\|_{L^{p}\left(B_{R} \times[0, T]\right)}+\right\| \partial_{t} u \|_{L^{p}\left(B_{R} \times[0, T]\right)} \\
& +\left\|\nabla_{x} u\right\|_{L^{p}\left(B_{R} \times[0, T]\right)}+\sum_{i, j=1}^{d}\left\|\partial_{i} \partial_{j} u\right\|_{L^{p}\left(B_{R} \times[0, T]\right)} .
\end{aligned}
$$

В этом пункте мы распространим результаты о локальной регулярности из предыдущего раздела на параболический случай. Доказательства приведенных здесь результатов даны в [9]. Начнем опять с существования плотностей.

Пусть $\Omega_{T}=\Omega \times(0, T), T>0$, и $A(\cdot, \cdot)=\left(a^{i j}(\cdot, \cdot)\right)_{i, j=1}^{d}-$ борелевское отображение на $\Omega_{T}$ со значениями в пространстве неотрицательных симметричных операторов в $\mathbb{R}^{d}$.

ТЕОРема 2.2.1. Пусть $\mu$ - локально конечная борелевская мера на $\Omega_{T}$, для которой $a^{i j} \in L_{\mathrm{loc}}^{1}\left(\Omega_{T}, \mu\right) u$

$$
\int_{\Omega_{T}}\left[\partial_{t} \varphi+a^{i j} \partial_{i} \partial_{j} \varphi\right] d \mu \leqslant C\left(\sup _{\Omega_{T}}|\varphi|+\sup _{\Omega_{T}}\left|\nabla_{x} \varphi\right|\right)
$$

для всех неотрищательных $\varphi \in C_{0}^{\infty}\left(\Omega_{T}\right)$. Тогда справедливы следующие утверждения.

(i) Если мера $\mu$ неотрицательна, то $(\operatorname{det} A)^{1 /(d+1)} \mu=\varrho d x d t$, причем $\varrho \in$ $L_{\text {loc }}^{(d+1)^{\prime}}\left(\Omega_{T}\right)$.

(ii) Если на каждом компакте в $\Omega_{T}$ отображение А равномерно ограничено, равномерно невырождено и гёльдерово по $x$ равномерно относительно $t$, то $\mu=\varrho d x d t$, где $\varrho \in L_{\mathrm{loc}}^{r}\left(\Omega_{T}\right)$ для каждого $r \in\left[1,(d+2)^{\prime}\right)$.

СлеДСТвИЕ 2.2.2. Пусть $\mu$ - такая локально конечная борелевская мера на $\Omega_{T}$, что $a^{i j}, b^{i}, c \in L_{\mathrm{loc}}^{1}\left(\Omega_{T}, \mu\right) u$

$$
\int_{\Omega_{T}}\left[\partial_{t} \varphi+a^{i j} \partial_{i} \partial_{j} \varphi+b^{i} \partial_{i} \varphi+c \varphi\right] d \mu=0 \quad \forall \varphi \in C_{0}^{\infty}\left(\Omega_{T}\right) .
$$

Тогда справедливы утверждения (i) и (ii) теоремы 2.2.1. Кроме того, в случае (ii), если $J=\left[T_{0}, T_{1}\right] \subset(0, T), B$ - шар с компактным замыканием в $\Omega$ u $W$ - окрестность $\bar{B} \times J$ с компактным замыканием, то для каждого числа $r<(d+2)^{\prime}$ имеем

$$
\|\varrho\|_{L^{r}(B \times J)} \leqslant C(d, r, A, W)\left(|\mu|(W)+\|c\|_{L^{1}(W, \mu)}+\|b\|_{L^{1}(W, \mu)}\right),
$$

где $C(d, r, A, W)$ зависит только от $d, r$, гёльдеровых норм $a^{i j}$ относительно $x$ на $W, \inf _{W} \operatorname{det} A, \sup _{i, j} \sup _{W}\left|a^{i j}\right|$ и расстояния от $B \times J$ до дW. Аналогичное утверждение верно в случае (i). 
ЗАмЕЧАНИЕ 2.2.3. Предположим, что в ситуации следствия 2.2.2 мы дополнительно имеем $|b|+|c| \in L_{\text {loc }}^{p}\left(\Omega_{T}\right)$, где $p>r^{\prime}$. Тогда

$$
\|\varrho\|_{L^{r}(B \times J)} \leqslant C(d, r, A, W)\left(|\mu|(W)+\left(\|c\|_{L^{p}(W)}+\|b\|_{L^{p}(W)}\right)\|\varrho\|_{L^{p^{\prime}}(W)}\right) .
$$

ЗАмЕЧАниЕ 2.2.4. (i) Если существует диффузионный процесс $\xi=\left(\xi_{t}^{s, x}\right)$, заданный стохастическим дифференциальным уравнением

$$
d \xi_{t}^{s, x}=\sqrt{2 A\left(\xi_{t}^{s, x}, t\right)} d W_{t}+b\left(\xi_{t}^{s, x}, t\right) d t, \quad \xi_{s}^{s, x}=x,
$$

то все приведенные выше результаты применимы к переходным вероятностям $P(s, x ; t, d y)$ диффузии $\xi$. А именно, для всякого фиксированного $(s, x)$ мера $\mu=P(s, x ; t, d y) d t$ удовлетворяет уравнению $(2.2 .1)$ при $c=0$. Значит, для почти всякого $t$ мера $P(s, x ; t, d y)$ абсолютно непрерывна. Этот факт хорошо известен для локально ограниченного $b$ (см. [108; гл. II, §2], [106; гл. 7, 9]). Однако мера $P(s, x ; t, d y)$ может не быть абсолютно непрерывной для всех $t$. В работе [109] построен такой пример, что $b=0$ и отображение $A(x, t)$ равномерно непрерывно, равномерно ограничено и равномерно положительно, но для некоторого фиксированного $t$ мера $P(s, x ; t, d y)$ чисто сингулярна относительно меры Лебега для всех $s \in(0, t)$ и всех $x$ (похожий пример построен B [110]).

(ii) Стоит отметить, что Н. И. Портенко [111] использовал аналогичные предположения относительно $A$ и $b$ в исследовании обобщенных диффузионных процессов. В частности, в [111; гл. II] показано, что если $A$ равномерно ограничено, равномерно положительно и равномерно гёльдерово, а $b$ - такое измеримое векторное поле на $\mathbb{R}^{n}$, что $|b| \in L^{p}\left(\mathbb{R}^{d}\right)$ с некоторым $p>d+2$, то существует непрерывная функция $G(s, x, t, y), 0 \leqslant s<t \leqslant T, x, y \in \mathbb{R}^{d}$, являющаяся переходной вероятностной плотностью для непрерывного марковского процесса $\left(x(t), \mathscr{M}_{t}^{s}, P_{s, x}\right)$, где $P_{s, x}$ - вероятностная мера на $\sigma$-алгебре $\mathscr{M}_{t}^{s}$ на пространстве $\Omega$ непрерывных траекторий $x(\cdot):[0,+\infty) \rightarrow \mathbb{R}^{d}$, порожденной отображением вычисления $x(\cdot) \mapsto x(u)$ с $u \in[s, t], P_{s, x}\{x(s)=x\}=1$, причем $P_{s, x}$-почти наверное

$$
x(t)-x(s)=\int_{s}^{t} b(x(\tau), \tau) d \tau+\int_{s}^{t} \sqrt{A(x(\tau), \tau)} d w_{s}(\tau)
$$

с некоторым винеровским процессом $\left(w_{s}(t), \mathscr{M}_{t}^{s}, P_{s, x}\right)$.

(iii) Отметим также, что параболические уравнения, рассмотренные в следствии 2.2.2, использовались в [112] для изучения некоторых потоков вероятностных мер. Приведенные выше результаты дают абсолютную непрерывность таких мер. Более сильные свойства регулярности для них будут установлены ниже при некоторых дополнительных предположениях на коэффициенты.

Теперь будем предполагать, что функции $a^{i j}(x, t)$ непрерывны по $x$ равномерно по $t$, т. е.

$$
\lim _{\delta \rightarrow 0} \sup _{t} \sup _{|x-y| \leqslant \delta}\left|a^{i j}(x, t)-a^{i j}(y, t)\right|=0 .
$$

Заметим, что $a^{i j}$ имеет модификацию с таким свойством, если

$$
\sup _{t}\left\|a^{i j}(\cdot, t)\right\|_{W^{p, 1}\left(B_{R}\right)}<\infty, \quad \text { где } p>d .
$$


Tеорема 2.2.5. Пусть $d \geqslant 2, p>d, q \in\left[p^{\prime},+\infty\right)$. Пусть $A$ и $A^{-1}$ равномерно ограничены, причем выполнено (2.2.2). Предположим, что $\mu$ - такая конечная мера на $B_{R, T}$, что для некоторого $N>0$ мы имеем

$$
\left|\int\left[\partial_{t} \varphi+a^{i j} \partial_{i} \partial_{j} \varphi\right] d \mu\right| \leqslant N\left\|\nabla_{x} \varphi\right\|_{L^{q}\left(B_{R, T}\right)} \quad \forall \varphi \in C_{0}^{\infty}\left(B_{R, T}\right) .
$$

Тогда $\mu \in \mathbb{H}^{q^{\prime}, 1}\left(B_{R^{\prime}},\left[t_{0}, t_{1}\right]\right) u \mu \in \mathscr{H}^{q^{\prime}, 1}\left(B_{R^{\prime}},\left[t_{0}, t_{1}\right]\right)$ для всех $R^{\prime}<R$ и всех отрезков $\left[t_{0}, t_{1}\right] \subset(0, T)$.

Следует отметить, что в доказательстве этого результата в [9; теорема 2.7] есть некоторая неточность: в самом начале утверждается, что умножением решения на гладкую функцию $\zeta$ с компактным носителем легко перейти к случаю решений с компактным носителем. В принципе это верно, но не непосредственно и требует некоторой дополнительной работы, поскольку неравенство для произведения будет несколько иного вида (в частности, появится член с интегралом от $\langle\nabla \zeta, \nabla \varphi\rangle$ относительно $\mu)$. Однако случай изначально компактного носителя достаточен для последующих применений этого результата в [9]. Тем не менее, если желать обосновать этот результат так, как он приведен, необходимо сначала применить предыдущие результаты и параболическую теорему вложения с целью обеспечить принадлежность плотности $\varrho$ решения к $L_{\mathrm{loc}}^{q^{\prime}}$ (это делается в несколько итераций) и только затем умножать на функции с компактным носителем; как только требуемая интегрируемость $\varrho$ установлена, член с $\langle\nabla \zeta, \nabla \varphi\rangle$ легко оценивается по неравенству Гёльдера, что дает для $\zeta \cdot \mu$ оценку типа $(2.2 .3)$.

ТЕОРема 2.2.6. Пусть А ограничено и локально равномерно невырождено на $B_{R, T}$ и выполнено $(2.2 .2)$, где теперь мы предположим, что $p>d+2$. Пусть $\mu$ - такая конечная борелевская мера на $B_{R, T}$, что $\mu \in L^{r}\left(B_{R, T}\right)$ nри некотором $r>p^{\prime}$. Пусть $\beta \in L^{p}\left(B_{R, T}\right)$. Предположим, что для всех функций $\varphi \in C_{0}^{\infty}\left(B_{R, T}\right)$ мы имеем

$$
\left|\int\left[\partial_{t} \varphi+a^{i j} \partial_{i} \partial_{j} \varphi\right] d \mu\right| \leqslant \int\left(|\varphi|+\left|\nabla_{x} \varphi\right|\right)|\beta \mu| d x .
$$

Тогда $\mu$ имеет плотность, которая локально гёлъдерова на $B_{R} \times(0, T)$ и входит в классы $\mathbb{H}^{p, 1}\left(B_{R^{\prime}},\left[T_{0}, T_{1}\right]\right)$ и $\mathscr{H}^{p, 1}\left(B_{R^{\prime}},\left[T_{0}, T_{1}\right]\right)$ для всех $R^{\prime}<R$ и всех отрезков $\left[T_{0}, T_{1}\right] \subset(0, T)$.

СлеДСТВИЕ 2.2.7. Пусть $p>d+2$ и А локально равномерно ограничено и локалъно равномерно невырождено на $\Omega_{T}$, причем условие (2.2.2) выполнено c $p>d+2$ для каждого шара $B_{R}$ с замыканием в $\Omega$. Пусть $b^{i}, c \in L_{\mathrm{loc}}^{p}\left(\Omega_{T}\right)$. Предположим, что $\mu$ - такая локально конечная знакопеременная борелевская мера на $\Omega_{T}$, что $b^{i}, c \in L_{\mathrm{loc}}^{1}\left(\Omega_{T}, \mu\right) u$

$$
\int_{\Omega_{T}}\left[\partial_{t} \varphi+a^{i j} \partial_{i} \partial_{j} \varphi+b^{i} \partial_{i} \varphi+c \varphi\right] d \mu=0 \quad \forall \varphi \in C_{0}^{\infty}\left(\Omega_{T}\right) .
$$

Тогда $\mu$ имеет локально гёльдерову плотность, которая принадлежит пространствам $\mathbb{H}^{p, 1}(U, J)$ и $\mathscr{H}^{p, 1}(U, J)$ для любых интервала $J$ и открытого множества $U$ таких, что $U \times J$ имеет компактное замыкание в $\Omega_{T}$. 
Стоит отметить, что если мера $\mu$ неотрицательна, то по неравенству Харнака непрерывная версия ее плотности строго положительна в каждой компоненте $\Omega_{T}$, в которой она не равна нулю тождественно. Более подробно это будет рассмотрено в п. 2.4 .

СлЕДСТвиЕ 2.2.8. Предположим, что для всех $\varphi \in C_{0}^{\infty}\left(\Omega_{T}\right)$ мы имеем

$$
\int_{\Omega_{T}}\left[\partial_{t} \varphi+a^{i j} \partial_{i} \partial_{j} \varphi+b^{i} \partial_{i} \varphi+c \varphi\right] d \mu=\int_{\Omega_{T}} f^{i} \partial_{i} \varphi d x d t
$$

где $f^{i} \in L_{\mathrm{loc}}^{p}\left(\Omega_{T}\right)$ и $a^{i j}, b^{i}$, с удовлетворяют тем же предположениям, что и в следствии 2.2.7. Тогда утверждение следствия 2.2.7, в котором (2.2.4) заменяется на (2.2.5), остается в силе.

СлЕДСТвИЕ 2.2.9. Предположим, что мера $\mu$ удовлетворяет условиям следствия 2.2.7, $B_{R_{0}}$ - открытый шар с замыканием в $\Omega u\left[t_{1}, t_{2}\right] \subset(0, T)$. Тогда для каждого отрезка $\left[\tau_{1}, \tau_{2}\right] \subset\left(t_{1}, t_{2}\right)$ и всякого $R<R_{0}$ существует такая постоянная $N$, зависящая от

$$
\begin{gathered}
t_{1}, t_{2}, \tau_{1}, \tau_{2}, R_{0}, R,\|c\|_{L^{p}\left(B_{R_{0}} \times\left[t_{1}, t_{2}\right]\right)}, \\
\inf _{B_{R_{0}} \times\left[t_{1}, t_{2}\right]} \operatorname{det} A, \sup _{t \in\left[t_{1}, t_{2}\right]}\left\|a^{i j}(\cdot, t)\right\|_{W^{p, 1}\left(B_{R_{0}}\right)}, \quad\left\|b^{i}\right\|_{L^{p}\left(B_{R_{0}} \times\left[t_{1}, t_{2}\right]\right)},
\end{gathered}
$$

что $N$ есть локально ограниченная функиия упомянутых величин $и$

$$
\|\mu\|_{\mathbb{H}^{p, 1}\left(B_{R},\left[\tau_{1}, \tau_{2}\right]\right)} \leqslant N\|\mu\|_{L^{p^{\prime}}\left(B_{R_{0}} \times\left[t_{1}, t_{2}\right]\right)} .
$$

Более того, можно выбрать $N$ так, что в случае неотрицательной меры $\mu$ мы получим

$$
\|\mu\|_{\mathbb{H}^{p, 1}\left(B_{R},\left[\tau_{1}, \tau_{2}\right]\right)} \leqslant N\|\mu\|_{L^{1}\left(B_{R_{0}} \times\left[t_{1}, t_{2}\right]\right)} .
$$

ЗАмечАниЕ 2.2.10. Предположение $p>d+2$ было использовано в доказательстве теоремы 2.2.6, чтобы улучшить исходную интегрируемость решения, а также обеспечить гёльдеровость (по подходящей теореме вложения). Из первого шага этого доказательства (или из теоремы 2.2.5) видно, что если мы предположим только, что $p>d$, то мы получим, что $\mu \in \mathbb{H}^{s, 1}\left(B_{R^{\prime}},\left[T_{0}, T_{1}\right]\right)$ и $\mu \in \mathscr{H}^{s, 1}\left(B_{R^{\prime}},\left[T_{0}, T_{1}\right]\right)$ при $s=p r /(p+r)>d$, при условии, что $r>p d /(p-d)$.

Приведем теперь модификацию теоремы 2.2.6, полученную в [23], которая отличается тем, что условие интегрируемости на коэффициент $\beta_{2}$ (отсутствующий в предыдущей теореме) выражено в терминах меры $\mu$, а не в терминах меры Лебега.

Теорема 2.2.11. Пусть А локально ограничено и локально равномерно невырождено на $B_{R, T}$, причем выполнено (2.2.2), где мы теперь предполагаем, что $p>d+2$. Пусть $\mu-$ конечная борелевская мер на $B_{R, T}$ с плотностью $\varrho \in L^{r}\left(B_{R, T}\right)$ при некотором $r>p^{\prime}$. Пусть $\beta_{1} \in L_{\mathrm{loc}}^{p}\left(B_{R, T}\right) u \beta_{2} \in L_{\mathrm{loc}}^{p}(\mu)$. Предположим, что для всех $\varphi \in C_{0}^{\infty}\left(B_{R, T}\right)$ мы имеем

$$
\left|\int_{B_{R, T}}\left[\partial_{t} \varphi+a^{i j} \partial_{x_{i}} \partial_{x_{j}} \varphi\right] d \mu\right| \leqslant \int_{B_{R, T}}\left(|\varphi|+\left|\nabla_{x} \varphi\right|\right)\left(\left|\beta_{1} \varrho\right|+\left|\beta_{2} \varrho\right|\right) d x d t .
$$




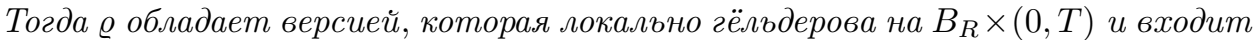
в классы $\mathbb{H}^{p, 1}\left(B_{R^{\prime}},\left[T_{0}, T_{1}\right]\right)$ и $\mathscr{H}^{p, 1}\left(B_{R^{\prime}},\left[T_{0}, T_{1}\right]\right)$ для каждого $R^{\prime}<R$ и каждого $\left[T_{0}, T_{1}\right] \subset(0, T)$.

СлеДСТВИЕ 2.2.12. Пусть $p>d+2, A$ и $A^{-1}$ локально равномерно ограничены на $\Omega_{T} u(2.2 .2)$ выполнено при некотором $p>d+2$ для каждого шара $B_{R}$ с замыканием в $\Omega$. Предположим, что $\mu$ - такая локально конечная знакопеременная борелевская мера на $\Omega_{T}$, что $b^{i}, c \in L_{\mathrm{loc}}^{p}(\mu) u$

$$
\int_{\Omega_{T}}\left[\partial_{t} \varphi+a^{i j} \partial_{x_{i}} \partial_{x_{j}} \varphi+\partial_{x_{i}} a^{i j} \partial_{x_{j}} \varphi+b^{i} \partial_{x_{i}} \varphi+c \varphi\right] d \mu=0 \quad \forall \varphi \in C_{0}^{\infty}\left(\Omega_{T}\right) .
$$

Тогда н обладает локально гёльдеровой плотностъю, которая принадлежит пространствам $\mathbb{H}^{p, 1}(U, J)$ и $\mathscr{H}^{p, 1}(U, J)$ для каждого интервала $J$ и каждого открытого множества $U$ таких, что замыкание $U \times J$ компактно в $\Omega_{T}$.

Вернемся к стохастическому уравнению (2.1.4). Известно, что если $A$ и $b$ ограничены, $A$ имеет две ограниченных производных и невырождено на $\mathbb{R}^{d}$, a $b$ имеет ограниченную производную, то существует диффузионный процесс $\xi_{t}^{x}$, удовлетворяющий (2.1.4) с $\xi_{0}^{x}=x, x \in \mathbb{R}^{d}$. Его переходные вероятности $P(t, x, d y)$ имеют плотности $p(t, x, y)$, удовлетворяющие следующим уравнениям:

$$
\begin{aligned}
\frac{\partial}{\partial t} p(t, x, y) & =a^{i j} \frac{\partial^{2}}{\partial x_{i} \partial x_{j}} p(t, x, y)+b^{i} \frac{\partial}{\partial x_{i}} p(t, x, y), \\
\frac{\partial}{\partial t} p(t, x, y) & =\frac{\partial^{2}}{\partial y_{i} \partial y_{j}}\left(a^{i j} p(t, x, y)\right)-\frac{\partial}{\partial y_{i}}\left(b^{i} p(t, x, y)\right)
\end{aligned}
$$

для всех $t>0, x, y \in \mathbb{R}^{d}$; кроме того, $P(0, x, d y)=\delta_{x}$. Это означает, что меры $P(t, x, d y) d t$ удовлетворяют (2.1.2). Отметим, что приведенные уравнения имеют очень много названий в литературе; в частности, они называются уравнениями Фоккера-Планка, Фоккера-Планка-Колмогорова, прямыми и обратными уравнениями Колмогорова и т.д. (сам А.Н. Колмогоров в [2] оба уравнения называл уравнениями Фоккера-Планка). Во избежание путаницы мы будем называть в этом обзоре (2.2.6) уравнением Колмогорова, а (2.2.7) уравнением Фоккера-Планка. Переходная полугруппа $\left(T_{t}\right)_{t \geqslant 0}$ процесса $\xi_{t}^{x}$ задана на $C_{b}\left(\mathbb{R}^{d}\right)$ формулой

$$
T_{t} f(x)=\int f(y) P(t, x, d y) .
$$

Если вероятностная мера $\mu$ инвариантна для $\left(T_{t}\right)_{t \geqslant 0}$, то $\mu$ удовлетворяет эллиптическому уравнению $L_{A, b}^{*} \mu=0$, и, обратно, если $\mu$ удовлетворяет этому стационарному уравнению, то она инвариантна для $\left(T_{t}\right)_{t \geqslant 0}$. Как мы знаем из пп. 1.5, 1.6, такой равносильности нет в случае неограниченных коэффициентов, даже если имеется наша полугруппа $\left(T_{t}^{\mu}\right)_{t \geqslant 0}$. Объясним, как можно рассматривать аналоги уравнений $(2.2 .6),(2.2 .7)$ для этой полугруппы в ситуации теоремы 1.7.5. Чтобы получить функцию трех аргументов $(t, x, y)$, возьмем $K_{t}^{*} \delta_{x}$, где $\delta_{x}-$ мера Дирака в $x$. Следующий результат показывает, что $K_{t}^{*} \delta_{x}=p_{A, b}(t, x, y) d y$ при $t>0$, где $p_{A, b}-$ функция из теоремы 1.7 .5$, и что имеет место аналог уравнения (2.2.7). 
Теорема 2.2.13. Предположим, что в ситуащии теоремы 1.7 .5 дана ограниченная мера $\mu_{0}$ на $\Omega$ и

$$
K_{t}^{*} \mu_{0}(d y):=\int_{\Omega} K_{t}(x, d y) \mu_{0}(d x)=\int_{\Omega} p_{A, b}(t, x, y) \mu_{0}(d x) d y .
$$

Тогда мера $K_{t}^{*} \mu_{0}(d y) d t$ удовлетворяет (2.1.2) для всех $T>0$. В частности, $K_{t}^{*} \mu_{0}$ имеет положительную непрерывную плотность из $W_{\mathrm{loc}}^{p, 1}(\Omega)$.

ЗАмЕчАниЕ 2.2.14. Из доказательства в [9] вытекает, что для каждого компакта $E \subset \Omega \times(0,+\infty)$ существует такая постоянная $C(E)$, что

$$
\sup _{(x, t) \in E} \sup _{y \in \Omega} p_{t}(x, y) \leqslant C(E)
$$

где $p_{t}(x, y)$ - совместно непрерывная версия плотности Радона-Никодима меры $K_{t}(x, d y)$ относительно меры $\mu=\varrho d x$ из теоремы 1.7 .5 , т. е. $p_{A, b}(t, x, y)=$ $p_{t}(x, y) \varrho(y)$. Значит, непрерывная плотность меры $K_{t}^{*} \mu_{0}(d y)$ задается формулой

$$
\int_{\Omega} p_{t}(x, y) \mu_{0}(d x) \varrho(y)
$$

В работе [113] при более сильных предположениях на $A$ и $b$ получены полезные глобальные оценки и включения в параболические классы Соболева для переходной плотности $p_{A, b}(t, x, y)$ из теоремы 1.7.5. В работе [114] исследована ситуация, когда $\nabla_{x} \varrho(x, t)$ существует даже для не обязательно дифференцируемого коэффициента $A$.

Конечно, как только существование плотностей установлено, во многих случаях можно применять результаты, известные для функций (но заботясь особо о форме уравнения), в частности, результаты из [38], [115]-[117].

2.3. Верхние оценки плотностей. Здесь мы приведем верхние оценки плотностей в случае неограниченного сноса. С одной стороны, предположения и техника, применяемые в этой ситуации, значительно отличаются от используемых для получения гауссовского убывания плотностей в случае ограниченных или нулевых сносов (см. [118]-[122], а также [123], где использованы некоторые условия на $\operatorname{div} b)$. С другой стороны, идеи и методы, известные для ограниченных коэффициентов, оказываются весьма полезными и в рассматриваемой ситуации. Сначала мы обсудим случай, когда начальное распределение имеет достаточно хорошую плотность, а затем кратко прокомментируем случай дираковского начального распределения (т. е. фундаментальных решений или переходных плотностей), изученный в [124], [113], [125] при некоторых дополнительных предположениях. Доказательства приводимых результатов см. в [21].

Будем говорить, что неотрицательная мера $\mu_{0}$ имеет конечную энтропию, если $\mu_{0}$ имеет плотность $\varrho_{0}$ относительно меры Лебега, причем $\varrho_{0} \ln \varrho_{0} \in L^{1}\left(\mathbb{R}^{d}\right)$, где мы полагаем $0 \ln 0:=0$.

ТЕОрема 2.3.1. Предположим, что $\mu$, где каждая $\mu_{t}$ - вероятностная мера, удовлетворяет (2.1.1), (2.1.3). Пусть выполнены следующие условия:

(i) отображение $A$ равномерно ограничено, $A(x, t) \geqslant \alpha \cdot \mathrm{I}$ для некоторой постоянной $\alpha>0$ и функции $x \mapsto a^{i j}(x, t)$ липшицевы с постоянной $\lambda$,

(ii) $|b| \in L^{2}(\mu)$. 
Предположим также, что функиия $\Lambda(x):=\ln \max (|x|, 1)$ входит в $L^{2}(\mu)$ (что выполнено, если, например, $\langle b(x, t), x\rangle \leqslant C_{1}|x|^{2} \Lambda(x)+C_{2}$ с некоторыми постоянными $C_{1}$ и $C_{2}$ и $\left.\Lambda \in L^{2}\left(\mu_{0}\right)\right)$. Если $\mu_{0}$ имеет конечную энтропию, то $\mu_{t}=\varrho(\cdot, t) d x$, где $\varrho(\cdot, t) \in W_{\mathrm{loc}}^{1,1}\left(\mathbb{R}^{d}\right)$ и для кажсдого $\tau<1$ мы имеем

$$
\int_{0}^{\tau} \int_{\mathbb{R}^{d}} \frac{|\nabla \varrho(x, t)|^{2}}{\varrho(x, t)} d x d t<\infty .
$$

В частности, $\sqrt{\varrho} \in \mathbb{H}^{2,2}\left(\mathbb{R}^{d} \times[0, \tau]\right)$ и $\varrho \in L^{d /(d-2), 1}\left(\mathbb{R}^{d} \times[0, \tau]\right)$, если $d>2$, $u \varrho \in L^{s, 1}\left(\mathbb{R}^{d} \times[0, \tau]\right)$ для всех $s \in[1, \infty)$, если $d=2$.

Если же мы имеем

$$
\limsup _{t \rightarrow 1} \int_{\mathbb{R}^{d}} \varrho(x, t) \Lambda(x) d x<\infty,
$$

что выполнено, например, если $\langle b(x, t), x\rangle \leqslant C_{1}|x|^{2}+C_{2}$ с некоторыми постоянными $C_{1}$ и $C_{2}$ и $\Lambda \in L^{1}\left(\mu_{0}\right)$, то (2.3.1) верно для $\tau=1$.

Доказательство в [21] дает полезную оценку

$$
\begin{aligned}
& \int_{0}^{\tau} \int_{\mathbb{R}^{d}} \frac{|\nabla \varrho|^{2}}{\varrho} d x d t \leqslant \alpha^{-2}\left(\|b\|_{2, \mu}+\lambda d^{3 / 2} \sqrt{\gamma}\right)^{2} \\
& \quad+2 \alpha^{-1} \ln 2+2 \alpha^{-1} \int_{\mathbb{R}^{d}} \varrho_{0}(x) \ln \varrho_{0}(x) d x+2 \alpha^{-1}(d+1) \int_{\mathbb{R}^{d}} \varrho(x, \tau) \Lambda(x) d x .
\end{aligned}
$$

ЗАмЕчАНИЕ 2.3.2. Из доказательства в [21] видно, что вместо интегрируемости $\varrho(x, 0) \ln \varrho(x, 0)$ достаточно потребовать только интегрируемость функции $\varrho(x, 0) \max (0, \ln \varrho(x, 0))$. Последнее приводит к тому, что в оценке $(2.3 .2)$ вместо функции $\varrho(x, 0) \ln \varrho(x, 0)$ появится $\varrho(x, 0) \max (0, \ln \varrho(x, 0))$. Однако полученные оценки и (2.1.10) показывают, что если сохранить все другие предположения, то энтропия $\varrho(x, 0)$ в любом смысле конечна. Однако если не требовать $\mu$-интегрируемость $\Lambda$, то положение может измениться. Например, если $d=1$, $b=0$ и $a=1 / 2$, то для всякого начального распределения $\mu_{0}$ решение дается сверткой $\mu_{0} * g_{t}$, где $g_{t}(x)=(2 \pi t)^{-1 / 2} \exp \left(-x^{2} /(2 t)\right)$. Если $\mu_{0}$ имеет такую плотность $\varrho_{0}$, что $\left|\varrho_{0}^{\prime}\right|^{2} / \varrho_{0} \in L^{1}\left(\mathbb{R}^{1}\right)$, но функция $\varrho_{0} \ln \varrho_{0}$ не интегрируема, то решение $\varrho(x, t)$ не имеет конечной энтропии для всех $t$, хотя величины

$$
\int\left|\partial_{x} \varrho(x, t)\right|^{2} \varrho(x, t)^{-1} d x
$$

равномерно ограничены. Этот же пример показывает, что для выполнения оценки (2.3.1) некоторые условия на начальное распределение необходимы. Достаточно взять в качестве $\mu_{0}$ меру Дирака в нуле. Тогда функция $\left|\partial_{x} \varrho\right|^{2} / \varrho$ не интегрируема на $\mathbb{R}^{1} \times(0,1)$. О достаточных условиях на $A$ и $b$, обеспечивающих конечную энтропию $\varrho(\cdot, t)$ для $t>0$ и дираковского начального распределения, упомянуто в конце пункта.

В примере 2.3.9 ниже можно найти условия на коэффициенты $A$ и $b$, обеспечивающие включение $|b| \in L^{2}(\mu)$.

Оценку (2.3.2) можно улучшить при дополнительных условиях на $A$ и $b$.

Положим

$$
b_{0}:=\left(b_{0}^{j}\right), \quad b_{0}^{j}=b^{j}-\partial_{x_{i}} a^{i j} .
$$


Теорема 2.3.3. Предположим, что мера $\mu$ удовлетворяет (2.1.1), (2.1.3), где $\nu=\varrho_{0} d x, \varrho_{0}$ имеет конечную энтропию и локально гёльдерова. Пусть $A$ u b удовлетворяют условиям (CP1) $и(\mathrm{CP} 2)$ с некоторым $p>d+2$. Предположим, что $\left|A^{-1 / 2} b_{0}\right| \in L^{2}(\mu), \ln (1+|x|) \in L^{4}(\mu)$ и что

$$
\liminf _{r \rightarrow \infty} \int_{0}^{1} \int_{r \leqslant|x| \leqslant 2 r}\left[r^{-4}\|A(x, t)\|^{2}+r^{-2} \Theta_{A}(x, t)^{2}\right] \mu_{t}(d x) d t=0 .
$$

Тогда $\varrho(\cdot, t) \in W_{\mathrm{loc}}^{p, 1}\left(\mathbb{R}^{d}\right)$ и для почти всех $\tau \in[0,1]$ имеем

$$
\begin{aligned}
& \int_{0}^{\tau} \int_{\mathbb{R}^{d}}\left|\frac{\sqrt{A} \nabla \varrho}{\varrho}\right|^{2} d \mu \\
& \quad \leqslant \int_{0}^{\tau} \int_{\mathbb{R}^{d}}\left|A^{-1 / 2} b_{0}\right|^{2} d \mu+2 \int_{\mathbb{R}^{d}}[\varrho(0, x) \ln \varrho(0, x)-\varrho(x, \tau) \ln \varrho(x, \tau)] d x
\end{aligned}
$$

причем правая часть конечна. При дополнительном предположении $A \geqslant \alpha \cdot \mathrm{I}$ для некоторого $\alpha>0$ имеем $\sqrt{\varrho} \in \mathbb{H}^{2,2}\left(\mathbb{R}^{d} \times[0,1]\right), \varrho \in L^{d /(d-2), 1}\left(\mathbb{R}^{d} \times[0,1]\right)$, если $d>2, u \varrho \in L^{s, 1}\left(\mathbb{R}^{d} \times[0,1]\right)$ для всех $s \in[1, \infty)$, если $d=2$.

ЗАмечание 2.3.4. Если $A$ равномерно ограничено, то условие $\ln (1+|x|) \in$ $L^{4}(\mu)$ можно ослабить до $\ln (1+|x|) \in L^{2}(\mu)$.

ТЕОРема 2.3.5. Предположим, что при условиях теоремы 2.3.1 мы дополнительно имеем

$$
\sup _{t \in[0,1]}\|b(\cdot, t)\|_{L^{d}\left(\mu_{t}\right)}<\infty
$$

$u \mu_{0}=\varrho(\cdot, 0) d x$, где $\varrho(\cdot, 0) \in L^{p}\left(\mathbb{R}^{d}\right)$ для всех $p \in[1,+\infty)$. Тогда

$$
\int_{0}^{\tau}\left(\int_{\mathbb{R}^{d}}|\varrho(x, t)|^{p} d x\right)^{q / p} d t<\infty
$$

для всех $p, q \in[1,+\infty)$ u $\tau \in(0,1)$.

Теорема 2.3.6. Предположим, что при условиях теоремы 2.3 .1 для некоторого $\beta>d+2$ мъ имеем $|b| \in L^{\beta}(\mu)$ и $\varrho(\cdot, 0) \in L^{\infty}\left(\mathbb{R}^{d}\right)$. Предположим также, что либо

$$
\sup _{t \in[0,1]}\|b(\cdot, t)\|_{L^{d}\left(\mu_{t}\right)}<\infty,
$$

либо $\varrho \in L^{p}\left(\mathbb{R}^{d} \times[0, \tau]\right)$ для всех $\tau<1$ при некотором $p>1$. Тогда справедливо включение $\varrho \in L^{\infty}\left(\mathbb{R}^{d} \times[0, \tau]\right)$ для каждого $\tau<1$.

ЗАмЕчАниЕ 2.3.7. (i) Ввиду замечания 2.3.2 интегрируемость функции $\varrho(x, 0) \ln \varrho(x, 0)$ в последних двух теоремах не требуется, поскольку интегрируемость функции $\varrho(x, 0) \max (0, \ln \varrho(x, 0))$ вытекает из включения $\varrho(\cdot, 0) \in L^{p}\left(\mathbb{R}^{d}\right)$ при $p>1$.

(ii) Предположение в теореме 2.3.5 и теореме 2.3.6, что интегралы от $\varrho(x, t)$ по $x$ равны 1 , можно заменить предположением, что эти интегралы равномерно ограничены.

(iii) Если в теореме 2.3.6 заранее дано, что $\varrho \in L^{p}\left(\mathbb{R}^{d} \times[0, \tau]\right)$ для некоторого $p>1$, то не требуется интегрируемость функции $|\ln (1+|x|)|^{2} \varrho(x, t)$, но ограниченность $\varrho(x, 0)$ важна. 
Эти теоремы позволяют получить глобальные верхние оценки на $\varrho$. Как и в эллиптическом случае, рассмотренном выше, идея проста: чтобы получить поточечную оценку $\varrho(x, t) \leqslant \Phi(x, t)^{-1}$, надо рассмотреть меру $\nu:=\Phi \cdot \mu$ и установить ограниченность ее плотности. Мы будем рассматривать функции $\Phi$, не зависящие от $t$. Если $\Phi$ имеет локально ограниченные первые и вторые производные, то мера $\nu$ удовлетворяет уравнению

$$
L^{*} \nu=\left(a^{i j} \partial_{x_{i}} \partial_{x_{j}} \Phi\right) \varrho+2 \partial_{x_{i}} \Phi \partial_{x_{j}}\left(a^{i j} \varrho\right)-b^{i} \partial_{x_{i}} \Phi \varrho=-L \Phi \cdot \varrho+2 \partial_{x_{j}}\left(a^{i j} \partial_{x_{i}} \Phi \varrho\right),
$$

понимаемому в том же смысле, что и (2.1.1).

Теорема 2.3.8. Предположим, что выполнены все условия теоремы 2.3.6 и дана такая функиия $\Phi \geqslant c>0 н а \mathbb{R}^{d}$ с локально ограниченными вторыми производными, что $\varrho(0, x) \leqslant C \Phi(x)^{-1}, \Phi \in L^{1}\left(\mu_{0}\right) u$

$$
\Phi^{1+\varepsilon},|L \Phi|^{\beta / 2} \Phi^{1-\beta / 2},|A \nabla \Phi|^{\beta} \Phi^{1-\beta} \in L^{1}(\mu), \quad \sup _{t \in[0,1]} \int_{\mathbb{R}^{d}} \Phi(x) \varrho(x, t) d x<\infty
$$

при некотором $\varepsilon>0$. Тогда для каждого $\tau<1$ найдется такое число $C_{\tau}$, что

$$
\varrho(x, t) \leqslant C_{\tau} \Phi(x)^{-1} \quad \text { для почти всех }(x, t) \in \mathbb{R}^{d} \times[0, \tau] .
$$

Пример 2.3.9. Предположим, что $A$ и $A^{-1}$ равномерно ограничены, $A$ равномерно липшицево по $x$ и для некоторых $\beta>d+2, r>0, \varepsilon>0, K>0$ мы имеем

$$
\begin{gathered}
|b| \in L^{\beta}(\mu), \quad \exp \left((2 K+\varepsilon)|x|^{r}\right) \in L^{1}(\mu), \\
\sup _{t \in[0,1]} \int_{\mathbb{R}^{d}} \exp \left(K|x|^{r}\right) \varrho(x, t) d x<\infty .
\end{gathered}
$$

Пусть $\sup _{t \in[0,1]}\|b(\cdot, t)\|_{L^{d}\left(\mu_{t}\right)}<\infty$. Наконец, пусть функция $\exp \left(K|x|^{r}\right) \varrho(0, x)$ ограничена и интегрируема на $\mathbb{R}^{d}$. Тогда для каждого $\tau<1$ имеется такое число $C(\tau)$, что

$$
\varrho(x, t) \leqslant C(\tau) \exp \left(-K|x|^{r}\right), \quad(x, t) \in \mathbb{R}^{d} \times[0, \tau] .
$$

Чтобы обеспечить условие $(2.3 .3)$ и указанные условия на $b$ и $\varrho(\cdot, 0)$, достаточно иметь оценки

$$
|b(x, t)| \leqslant C \exp \left(2 K \beta^{-1}|x|^{r}\right), \quad \varrho(0, x) \leqslant C \exp \left(-K^{\prime}|x|^{r}\right)
$$

с некоторым $K^{\prime}>K$ и оценку

$$
\langle x, b(x, t)\rangle \leqslant c_{1}-c_{2}|x|^{r}, \quad c_{2}>2 r K \sup _{t, x}\|A(x, t)\| .
$$

В самом деле, пусть функция $\Phi \in C^{2}\left(\mathbb{R}^{d}\right)$ такова, что $\Phi(x)=\exp \left(K|x|^{r}\right)$, если $|x| \geqslant 1$. Все условия теоремы 2.3 .8 выполнены. При условии (2.3.4) выберем $\delta \in(0, \varepsilon)$ так, что будет выполнено неравенство

$$
r(2 K+\delta) \sup _{t, x}\|A(x, t)\|<c_{2}
$$


и возьмем функцию $V \in C^{2}\left(\mathbb{R}^{d}\right)$, равную $\exp \left((2 K+\delta)|x|^{r}\right)$ при $|x| \geqslant 1$. Тогда для некоторого $c$ получим оценку $L V \leqslant c$. Из теорем существования в п. 2.6 ниже вытекает, что решение существует и нормы $\|V \varrho(\cdot, t)\|_{L^{1}\left(\mathbb{R}^{d}\right)}$ равномерно ограничены. Остающиеся предположения теоремы 2.3.8 также выполнены. Аналогичным образом при более слабых условиях можно получить степенную оценку.

При некоторых дополнительных предположениях в недавних работах [124], [113], [125] получены зависящие от времени оценки решений. Например, в [113] доказан следующий результат.

Tеорема 2.3.10. Пусть $A$ и $A^{-1}$ равномерно ограничены, $a^{i j} \in C^{1+\alpha}\left(\mathbb{R}^{d}\right)$, $b^{i} \in C_{\mathrm{loc}}^{\alpha}\left(\mathbb{R}^{d}\right)$,

$$
\limsup _{|x| \rightarrow \infty}|x|^{-\beta}\langle x, b(x)\rangle \leqslant-c, \quad|b(x)| \leqslant c_{1} \exp \left(c_{2}|x|^{\beta-\varepsilon}\right),
$$

где $c, c_{1}, c_{2}>0, \varepsilon>0$ и $\beta>2$. Тогда имеет место следующая оченка для фундаментальной плотности $p_{A, b}(t, x, y)$ из теоремъ 1.7.5:

$$
p_{A, b}(t, x, y) \leqslant c_{3} \exp \left(c_{4} t^{-\beta /(\beta-2)}\right) \exp \left(-\gamma|y|^{\beta}\right), \quad t \in(0,1), \quad x, y \in \mathbb{R}^{d} .
$$

Eсли $|b(x)| \leqslant c\left(1+|x|^{q}\right)$, mo $p_{A, b}(t, x, y) \leqslant c t^{-\sigma}(1+|y|)^{-\theta}$ при некоторых постоянных с, $\sigma, \theta>0$.

Аналогичные оценки установлены также для $\partial_{t} p_{A, b}, D_{y} p_{A, b}$ и $D_{y}^{2} p_{A, b}$ в случае, когда $a^{i j} \in C_{b}^{2}\left(\mathbb{R}^{d}\right), b^{i} \in C^{2}\left(\mathbb{R}^{d}\right)$ и $|D b(x)|,\left|D^{2} b(x)\right|$ допускают такую же оценку, как и $|b|$. Некоторые из этих оценок уточнены в [125] с использованием функций Ляпунова, зависящих от $t$. Например, предыдущая оценка уточнена до

$$
p_{A, b}(t, x, y) \leqslant c_{3} t^{-\kappa} \exp \left(-\delta t^{\alpha}|y|^{\beta}\right),
$$

где $\theta=\alpha k(\beta-1) / \beta-1, k>d+2, \alpha>\beta /(\beta-2), \delta<c /(\Lambda \beta), \Lambda=\sup _{x}\|A(x)\|$. Эти результаты были недавно усилены С. В. Шапошниковым.

Было бы интересно изучить поведение решений на бесконечности при рассмотренных предположениях на коэффициенты в случае, когда начальное распределение есть мера; имеется много результатов в этом направлении для решений в различных классах функций и обычно в случае ограниченных коэффициентов (см. обзор [126]).

2.4. Неравенство Харнака для параболических уравнений. Теперь рассмотрим неравенство Харнака для параболических уравнений. Пусть $\Omega-$ ограниченная область в $\mathbb{R}^{d}, Q=\Omega \times(0,1)$ и $A=\left(a^{i j}\right)_{1 \leqslant i, j \leqslant d}$ - измеримое матричнозначное отображение на $Q$ с $a^{i j}=a^{j i}$ такое, что существуют постоянные $\gamma \geqslant 0$ и $\alpha>0$, для которых

$$
\sum_{i, j}\left|a^{i j}(x, t)\right|^{2} \leqslant \gamma^{2} \quad \text { и } \quad A(x, t) \geqslant \alpha \cdot \text { I } \quad \text { для всех }(x, t) \in Q .
$$

Кроме того, пусть $b: Q \rightarrow \mathbb{R}^{d}$ - такое измеримое векторное поле, что

$$
\sup _{(x, t) \in Q}|b(x, t)| \leqslant B<\infty .
$$


Предположим, что неотрицательная функция $u \in \mathbb{H}^{2,1}(Q)$ удовлетворяет уравнению

$$
\partial_{t} u=\partial_{x_{i}}\left(a^{i j} \partial_{x_{j}} u-b^{i} u\right),
$$

т. е. для каждой функции $\varphi \in C_{0}^{1}(Q)$ имеет место равенство

$$
\iint_{Q}\left[-\partial_{t} \varphi u+\partial_{x_{i}} \varphi\left(a^{i j} \partial_{x_{j}} u-b^{i} u\right)\right] d x d t=0 .
$$

Из общей теории параболических уравнений (см., например, [115; гл. 3, теоремы 8.1 и 10.1]) следует, что при наших предположениях всякое решение $u$ имеет локально гёльдерову версию.

Зафиксируем точку $(\bar{x}, \bar{t}) \in \Omega \times(0,1]$. Пусть $R(\bar{x}, r)$ - открытый куб с ребром длины $r$ и центром в точке $\bar{x}$. Положим

$$
Q(r)=R(\bar{x}, r) \times\left(\bar{t}-r^{2}, \bar{t}\right), \quad Q^{*}(r)=R(\bar{x}, r) \times\left(\bar{t}-8 r^{2}, \bar{t}-7 r^{2}\right) .
$$

Справедлива следующая классическая теорема (см. работу [127; теорема 3], обобщающую результат Ю. Мозера [128]). Относительно методов получения неравенства Харнака см. также [119] и имеющуюся там библиографию.

TеОрема 2.4.1. Пусть $Q(3 r) \subset Q$. Тогда для непрерывной версии функиии и, удовлетворяющей уравнению (2.4.3), мы имеем

$$
\sup _{(x, t) \in Q^{*}(r)} u(x, t) \leqslant C \inf _{(x, t) \in Q(r)} u(x, t),
$$

где число $C=C(d, \alpha, \gamma, B r)$ зависит только от $d, \alpha, \gamma$ u $B r$.

Как и в эллиптическом случае, нас интересует более точная форма зависимости $C$ от указанных параметров из (2.4.1), (2.4.2). Приведенные ниже результаты доказаны в [22].

ТЕОРема 2.4.2. Пусть $Q(3 r) \subset Q$. Тогда для непрерывной версии функиии и выполнено следующее неравенство:

$$
\sup _{(x, t) \in Q^{*}(r)} u(x, t) \leqslant C \inf _{(x, t) \in Q(r)} u(x, t),
$$

¿əe

$$
C:=C(d, \alpha, \gamma, B, r):=\exp \left(c(d)\left[1+\alpha^{-1}+\left(\alpha^{-1 / 2}+\alpha^{-1}\right)(B r+\gamma)\right]^{2}\right) .
$$

Следующий результат уточняет полученную оценку в плане зависимости от $r$. Его преимущество по сравнению с предыдущей теоремой состоит в том, что теперь $B$ входит в оценку без множителя $r$.

Теорема 2.4.3. Предположим, что $B\left(z_{0}, \theta r\right) \subset \Omega$ для некоторых $\theta>1$ u $r>0$. Тогда при $0<s<t<1 u x, y \in B\left(z_{0}, r\right)$ для непрерывной версии функиии и выполнено следующее неравенство:

$$
u(y, s) \leqslant u(x, t) \exp \left(K\left(\frac{|x-y|^{2}}{t-s}+(B+1)^{2} \frac{t-s}{\delta^{2}}+1\right)\right),
$$

где $\delta=\min \{(\theta-1) r, \sqrt{s}\}$, а число $K$ зависит только от $d, \alpha$ и $\gamma$ следующим образом (с некоторым числом $c(d)$, зависящим только от $d)$ :

$$
K:=c(d)\left|1+\alpha^{-1}+\left(\alpha^{-1}+\alpha^{-1 / 2}\right) \gamma\right|^{2} .
$$


2.5. Нижние оценки плотностей. Перейдем теперь к нижним оценкам плотностей решений параболических уравнений для мер. Как мы увидим, имеются нижние оценки, похожие на верхние оценки, обсуждавшиеся в п. 2.3; однако различие между верхними и нижними оценками уже более существенно, чем в эллиптическом случае. Доказательства даны в [22].

Пусть $A=\left(a^{i j}\right)_{1 \leqslant i, j \leqslant d}$ - измеримое матричнозначное отображение на множестве $\mathbb{R}^{d} \times(0,1)$, причем матрица $A(x, t)$ положительно определена, и пусть $b-$ измеримое векторное поле на $\mathbb{R}^{d} \times(0,1)$ со значениями в $\mathbb{R}^{d}$. Как и в эллиптическом случае, в исследовании нижних оценок удобнее иметь дело с операторами дивергентного вида $\mathscr{L}=\mathscr{L}_{A, b}$.

Борелевская мера $\mu$ на $\mathbb{R}^{d} \times(0,1)$ удовлетворяет слабому параболическому уравнению (2.1.1), если функции $a^{i j}$ и $b^{i}$ интегрируемы на каждом компакте в $\mathbb{R}^{d} \times(0,1)$ относительно $\mu$ и для каждой функции $\varphi \in C_{0}^{\infty}\left(\mathbb{R}^{d} \times(0,1)\right)$ выполнено равенство

$$
\int_{\mathbb{R}^{d} \times(0,1)} \mathscr{L} \varphi d \mu=0,
$$

которое понимается одним из следующих двух способов.

(I) Для каждого отрезка $J \subset(0,1)$ и каждого шара $U \subset \mathbb{R}^{d}$ функции $a^{i j}$ входят в класс $\mathbb{H}^{1,1}(U \times J)$, функции $a^{i j}, \partial_{x_{i}} a^{i j}$ и $b^{i}$ борелевски измеримы и локально интегрируемы относительно $|\mu|$, причем выполнено равенство

$$
\int_{\mathbb{R}^{d} \times(0,1)}\left[\partial_{t} \varphi+a^{i j} \partial_{x_{i}} \partial_{x_{j}} \varphi+\partial_{x_{i}} a^{i j} \partial_{x_{j}} \varphi+b^{j} \partial_{x_{j}} \varphi\right] d \mu=0 .
$$

(II) Для каждого отрезка $J \subset(0,1)$ и каждого шара $U \subset \mathbb{R}^{d}$ ограничение меры $\mu$ на $U \times J$ имеет плотность $\varrho$ из класса $\mathbb{H}^{1,1}(U \times J)$, функции $a^{i j} \partial_{x_{i}} \varrho$ и $b^{i} \varrho$ локально интегрируемы по Лебегу, причем

$$
\int_{\mathbb{R}^{d} \times(0,1)}\left[\partial_{t} \varphi \varrho-a^{i j} \partial_{x_{i}} \varrho \partial_{x_{j}} \varphi+b^{i} \partial_{x_{i}} \varphi \varrho\right] d x d t=0 .
$$

Далее мы всегда указываем, какой из случаев рассматривается, ссылаясь на (2.5.1) в случае (I) и на (2.5.2) в случае (II). Наше предположение, что матрицы $A(x, t)=\left(a^{i j}(x, t)\right)_{i, j \leqslant d}$ симметричны и строго положительны, гарантирует абсолютную непрерывность меры $\mu$ в случае (I). По этой причине далее мы рассматриваем меры $\mu$, представленные в виде $\mu(d t d x)=\mu_{t}(d x) d t$ посредством семейства борелевских мер $\left(\mu_{t}\right)_{t \in(0,1)}$ на $\mathbb{R}^{d}$. В этом случае $(2.1 .1)$ можно записать как

$$
\int_{0}^{1} \int_{\mathbb{R}^{d}} \mathscr{L} \varphi(x, t) \mu_{t}(d x) d t=0,
$$

что понимается одним из двух способов, описанных выше. Следует отметить, что альтернативное предположение в случае (II), что $\mu$ имеет локально соболевскую плотность, выполнено автоматически, если в случае (I) коэффициенты $A$ и $b$ удовлетворяют некоторым дополнительным предположениям, указанным в п. 2.2. По этой причине в некоторых результатах мы рассматриваем решения, априорно имеющие локально соболевские плотности, и не делаем никаких предположений о регулярности $A$; однако в других результатах, имеющих дело 
с применениями к переходным вероятностям, мы требуем подходящую локальную соболевскую регулярность $A$, чтобы гарантировать соболевскую регулярность всех решений.

Пусть $V$ - непрерывная возрастающая функция на $[0, \infty)$ и $V(0)>0$.

TЕОРема 2.5.1. Пусть $\sup _{t \in(0,1)}|b(x, t)| \leqslant V(|x| / \theta)$ для почти всех $x \in \mathbb{R}^{d}$, где $\theta>1$. Пусть

$$
\alpha(r):=\sup _{t \in(0,1),|x| \leqslant r}\left\|A(x, t)^{-1}\right\|, \quad \gamma(r):=\sup _{t \in(0,1),|x| \leqslant r}\|A(x, t)\| .
$$

Пусть $\mu$ - такая неотрицательная мера с плотностъю $\varrho н а \mathbb{R}^{d} \times(0,1)$, что

$$
\varrho \in \mathbb{H}^{2,1}(U \times J)
$$

для всякого шара $U \subset \mathbb{R}^{d}$ и всякого отрезка $J$ в $(0,1)$. Предположим, что $\mu$ удовлетворяет уравнению (2.1.1) в смысле (2.5.2), т.е. имеем дело со случаем (II). Тогда существует такое положительное число $K=K(d)$, что непрерывная версия функиии @ удовлетворяет неравенству

$$
\begin{gathered}
\varrho(x, t) \geqslant \varrho(0, s) \exp \left(-K(d)\left|1+\alpha(\theta|x|)^{-1}+\left[\alpha(\theta|x|)^{-1}+\alpha(\theta|x|)^{-1 / 2}\right] \gamma(\theta|x|)\right|^{2}\right. \\
\left.\times\left(1+\frac{t-s}{s} V(|x|)^{2}+\frac{1}{t-s}|x|^{2}\right)\right),
\end{gathered}
$$

где $0<s<t<1, x \in \mathbb{R}^{d}$. В частности, если $\|A(x, t)\| \leqslant \gamma u\left\|A(x, t)^{-1}\right\| \leqslant \alpha$, то существует такое положительное число $K=K(d, \alpha, \gamma, \theta)$, что непре-

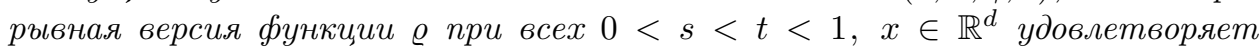
неравенству

$$
\varrho(x, t) \geqslant \varrho(0, s) \exp \left(-K\left(1+\frac{t-s}{s} V(|x|)^{2}+\frac{1}{t-s}|x|^{2}\right)\right) .
$$

СлЕДСТвиЕ 2.5.2. Предположим, что в ситуачии теоремы 2.5.1 мы имеем оченки

$$
\|A(x, t)\| \leqslant \gamma \quad u \quad\left\|A(x, t)^{-1}\right\| \leqslant \alpha
$$

$u$ что для почти всех $t \in(0,1)$ функиия $x \mapsto \varrho(x, t)$ не равна нулю тождественно. Тогда для каждого отрезка $\left[\tau_{1}, \tau_{2}\right]$ в $(0,1)$ существует такое число $K=K\left(d, \alpha, \gamma, \theta, \tau_{1}, \tau_{2}\right) \geqslant 0$, что для всех $t \in\left[\tau_{1}, \tau_{2}\right]$ и $x \in \mathbb{R}^{d}$ выполнено следующее неравенство:

$$
\exp \left(-K\left(1+V(|x|)^{2}+|x|^{2}\right)\right) \leqslant \varrho(x, t) \leqslant \exp \left(K\left(1+V(|x|)^{2}+|x|^{2}\right)\right) .
$$

ПримеР 2.5.3. Пусть в ситуации теоремы 2.5.1 матрицы $A(x, t)$ и $A(x, t)^{-1}$ равномерно ограничены и для некоторых постоянных $c_{1}>0$ и $c_{2}>0$ неравенства

$$
\sup _{t \in(0,1)}|b(x, t)| \leqslant c_{1}|x|^{\beta}+c_{2}
$$

выполнены для почти всех $x$. Тогда существует такое положительное число $K$, что

$$
\varrho(x, t) \geqslant \varrho(0, s) \exp \left(-K\left(1+\frac{t-s}{s}|x|^{2 \beta}+\frac{1}{t-s}|x|^{2}\right)\right), \quad s, t \in(0,1), \quad s<t .
$$


Например, если

$$
L=\partial_{t}+\frac{1}{2} \Delta
$$

то мера $(2 \pi t)^{-1 / 2} e^{-|x|^{2} /(2 t)} d x d t$ есть решение. Для заданного $\delta>0$ наши результаты дают такое число $K(\delta)>0$, что $\varrho \geqslant e^{-K(\delta)|x|^{2} / t}$ в полосе $\mathbb{R}^{d} \times(\delta, 1)$, где $\delta>0$. Аналогичным образом наши нижние оценки точны в случае линейного коэффициента сноса, но они становятся менее точными в случае квадратичного роста $|b|$; например, если $\varrho(x, t)=C \exp \left(-|x|^{3}\right)$, то в наших нижних оценках появляется $\exp \left(-K|x|^{4}\right)$.

Приведем условия на коэффициенты $A$ и $b$, обеспечивающие двусторонние экспоненциальные оценки плотности решения в параболическом случае.

ПримеР 2.5.4. Предположим, что $A(x, t)$ симметричны и положительны, $A(x, t)$ и $A(x, t)^{-1}$ равномерно ограничены, функции $x \mapsto a^{i j}(x, t)$ равномерно липшицевы с общей постоянной и что для некоторых $r>1, \sigma \geqslant 0, K>0$ и $K^{\prime}>K$ мы имеем

$$
\begin{array}{cc}
|b(x, t)| \leqslant C+C|x|^{r-1+\sigma}, & \varrho(x, 0) \leqslant C \exp \left(-K^{\prime}|x|^{r}\right), \\
\langle x, b(x, t)\rangle \leqslant c_{1}-c_{2}|x|^{r}, & c_{2}>2 r K \sup _{x, t}\|A(x, t)\| .
\end{array}
$$

Предположим, что вероятностная мера $\mu$ на $\mathbb{R}^{d} \times(0,1)$ удовлетворяет уравнению (2.1.1) в смысле (2.5.1), т. е. в случае (I). Тогда $\mu$ имеет такую непрерывную плотность $\varrho$, что для каждого отрезка $\left[\tau_{1}, \tau_{2}\right] \subset(0,1)$ существуют такие числа $C_{1}, C_{2}$ и $K_{0}$, что при всех $(x, t) \in \mathbb{R}^{d} \times\left[\tau_{1}, \tau_{2}\right]$ выполнены неравенства

$$
C_{1} \exp \left(-K_{0}|x|^{2 r+2 \sigma-2}-K_{0}|x|^{2}\right) \leqslant \varrho(x, t) \leqslant C_{2} \exp \left(-K|x|^{r}\right) .
$$

Верхняя оценка вытекает из п. 2.3, а нижняя оценка вытекает из изложенных выше результатов. В отличие от эллиптического случая, здесь нет совпадения степеней $|x|$ в нижних и верхних оценках. Заметим, что указанные условия дают также и существование решения $\mu=\mu_{t} d t$, где каждая $\mu_{t}$ - вероятностная мера, для произвольного начального распределения (см. п. 2.6). Проблема единственности также рассматривается в п. 2.6.

Еще одно приложение изложенных результатов связано с доказательством существования конечной энтропии всякого решения по пространственной переменной для всякого положительного $t$ при любом начальном распределении. Существование конечной энтропии, полезное во многих отношениях, необходимо для применения тех результатов из п. 2.3, которые дают интегрируемость $|\nabla \varrho(x, t)|^{2} / \varrho(x, t)$.

Пусть $W$ - непрерывная возрастающая функция на полупрямой $[0, \infty)$, причем $W(0)>0$ и $\lim _{r \rightarrow \infty} W(r)=+\infty$.

ПРЕДЛОЖЕНИЕ 2.5.5. Предположим, что матрицы $A(x, t)$ положительно определены и равномерно ограничены вместе с матрицами $A(x, t)^{-1}$ и что для некоторого $\theta>1$ выполнено следующее неравенство:

$$
\sup _{t \in(0,1)}|b(x, t)|^{2} \leqslant W\left(\frac{|x|}{\theta}\right), \quad x \in \mathbb{R}^{d} .
$$


Пусть $\mu$ - мера вида $\mu=\mu_{t} d t$, где каждая $\mu_{t}$ - вероятностная мера на $\mathbb{R}^{d}$, и пусть н удовлетворяют условию (2.5.3) и уравнению (2.1.1) в смысле (2.5.2). Предположим, что

$$
\int_{0}^{1} \int_{\mathbb{R}^{d}}\left[|x|^{2}+W(|x|)\right] \mu_{t}(d x) d t<\infty .
$$

Тогда для каждого отрезка $\left[\tau_{1}, \tau_{2}\right] \subset(0,1)$ мы имеем

$$
\int_{\tau_{1}}^{\tau_{2}} \int_{\mathbb{R}^{d}} \varrho(x, s)|\ln \varrho(x, s)| d x d s<\infty .
$$

СлеДСтвиЕ 2.5.6. Предположим, что а $a^{i j} \in \mathbb{H}_{\text {loc }}^{2,1}\left(\mathbb{R}^{d} \times(0,1)\right), A(x, t)$ положительно определень, $A(x, t)$ и $A(x, t)^{-1}$ равномерно ограничень. Кроме того, предположим, что для некоторого $\theta>1$ выполнено следующее неравенство:

$$
\sup _{t \in(0,1)}|b(x, t)|^{2} \leqslant W\left(\frac{|x|}{\theta}\right), \quad x \in \mathbb{R}^{d} .
$$

Пусть $\mu$-мера вида $\mu=\mu_{t} d t$, где каждая $\mu_{t}$ - вероятностная мера, причем для $\mu$ выполнено условие (2.5.3). Предположим, что н удовлетворяет уравнению (2.1.1) в смысле (2.5.2) и имеет такое начальное распределение $\mu_{0}$, что функиия $W_{0}(x):=W(|x|)$ интегрируема относительно $\mu_{0}$. Наконеи, пусть

$$
W(r) \geqslant c_{1}+c_{2} r^{2} \quad \text { u } \quad \mathscr{L} W_{0}(x) \leqslant C
$$

с некоторыми постоянными $c_{1}, c_{2}, C>0$. Тогда для непрерывной версии $\varrho$ плотности меры $\mu$ мы имеем

$$
\sup _{s \in(0,1)} \int_{\mathbb{R}^{d}} \varrho(x, s)|\ln \varrho(x, s)| d x<\infty .
$$

Подчеркнем еще раз, что если функции $x \mapsto a^{i j}(x, t)$ локально липшицевы равномерно по $t \in(0,1)$, то в теореме 2.5.1, следствии 2.5.2, примере 2.5.3, предложении 2.5.5 и следствии 2.5.6 всякое решение $\mu$ задачи (2.1.3) в смысле (2.5.1) есть решение также и в смысле (2.5.2).

ПримеР 2.5.7. Предположим, что в следствии 2.5.6 дополнительно известно, что функции $x \mapsto a^{i j}(x, t)$ равномерно липшицевы с общей постоянной. Тогда для каждого отрезка $\left[\tau_{1}, \tau_{2}\right] \subset(0,1)$ согласно п. 2.3 мы имеем

$$
\int_{\tau_{1}}^{\tau_{2}} \int_{\mathbb{R}^{d}} \frac{|\nabla \varrho(x, t)|^{2}}{\varrho(x, t)} d x d t<\infty .
$$

ПримеР 2.5.8. Предположим, что матрицы $A(x, t)$ и $A(x, t)^{-1}$ равномерно ограничены, функции $x \mapsto a^{i j}(x, t)$ равномерно липшицевы с общей постоянной и существуют такие числа $c, c_{0}, c_{1}, c_{2}, r>0$, что

$$
|b(x, t)| \leqslant c_{0} \exp \left(c|x|^{r}\right), \quad(b(x, t), x) \leqslant c_{1}-c_{2}|x|^{r}, \quad c_{2}>2 c r \sup _{x, t}\|A(x, t)\| .
$$


Пусть $\mu$ - вероятностная мера на $\mathbb{R}^{d} \times(0,1)$, удовлетворяющая уравнению $(2.1 .1)$ в смысле (2.5.1) с таким начальным условием $\mu_{0}$, что функция $\exp \left(c|x|^{r}\right)$ интегрируема относительно $\mu_{0}$. Тогда

$$
\int_{\tau_{1}}^{\tau_{2}} \int_{\mathbb{R}^{d}} \frac{|\nabla \varrho(x, t)|^{2}}{\varrho(x, t)} d x d t<\infty
$$

для каждого отрезка $\left[\tau_{1}, \tau_{2}\right] \subset(0,1)$. Чтобы это доказать, достаточно взять $W_{0}(z)=\exp \left(M|z|^{r}\right)$ при $M>2 c$, достаточно близком к $2 c$.

Теперь мы обратимся к нижним оценкам без условий на рост коэффициента сноса; доказательства можно найти в [129]. До конца этого пункта мы будем предполагать, что матрица $A(x, t)=\left(a^{i j}(x, t)\right)_{1 \leqslant i, j \leqslant d}$ симметрична и удовлетворяет следующим условиям:

(GP1) существует такая постоянная $\lambda>0$, что для всех $x, y \in \mathbb{R}^{d} u t \in$ $(0,1)$ мы имеем

$$
\left|a^{i j}(x, t)-a^{i j}(y, t)\right| \leqslant \lambda|x-y|
$$

(GP2) существуют такие постоянные $m, M>0$, что для всех $x, y \in \mathbb{R}^{d}$ $u t \in(0,1)$ мы имеем

$$
m|y|^{2} \leqslant \sum_{1 \leqslant i, j \leqslant d} a^{i j}(x, t) y_{i} y_{j} \leqslant M|y|^{2} .
$$

Если дополнительно к (GP1) и (GP2) мы имеем $b \in L_{\mathrm{loc}}^{p}(\mu)$ при некотором $p>d+2$, то решение $\mu$ обладает непрерывной плотностью $\varrho$, принадлежащей классу $\mathbb{H}^{p, 1}(U, J)$ для каждого шара $U$ и каждого интервала $J \subset(0,1)$.

Оказывается, что без всяких предположений о локальной ограниченности или локальной интегрируемости коэффициента $b$ относительно меры Лебега можно получить оценки вида

$$
\varrho(x, t) \geqslant e^{-f\left(c_{1}|x|^{2}+c_{2}\right)}, \quad x \in \mathbb{R}^{d}, \quad t \in J,
$$

где $J=\left[\tau_{1}, \tau_{2}\right] \subset(0,1)$ - произвольный отрезок, $c_{1}=c_{1}(J), c_{2}=c_{2}(J)-$ некоторые постоянные и $f \in C^{2}([0,+\infty))$ удовлетворяет следующим условиям:

(HP1) $f(z)>0, f^{\prime}(z)>0 u f^{\prime \prime}(z)>0$, если $z>0$;

(НР2) для некоторого $0<\gamma<1$ функиия $e^{-(1-\gamma) f(z)}$ выпукла (ее вторая производная неотрицательна) на $\left(z_{0},+\infty\right)$ для некоторого $z_{0} \geqslant 0$.

Чтобы получить оценку (2.5.4), достаточно в дополнение к условиям (GP1) и (GP2) потребовать выполнение следующих условий:

(GP3) $|b| \exp (\psi(|b|)) \in L^{p}(\mu)$, где $p>d+2 u \psi$ - такая неотрицательная строго возрастающая непрерьвная функиия на $[0, \infty)$, что для некоторого числа $N>0$ миь имеем

(НР3) $\psi^{-1}(z) \leqslant N \sqrt{f^{\prime}\left(f^{-1}(z)\right)}$ для всех $z>0$.

Согласно (НР1) и (НР3) имеем $f^{\prime}(y) \rightarrow \infty$. Значит, существует такое число $y_{0}>\max \left\{z_{0}, 1\right\}$, что $f^{\prime}(y) \geqslant 1$ и $\left(f^{\prime}(y)\right)^{-1 / 2} e^{f(y)} \geqslant e^{\psi(0)}$ при $y>y_{0}$. Пусть $\omega_{0}=e^{-f\left(\ln y_{0}\right)}$. Тогда $0<\omega_{0}<1$. Положим

$$
\Lambda=\min \left\{\omega_{0}\left(2\|\varrho\|_{L^{\infty}}\right)^{-1}, 1\right\} .
$$


Зафиксируем куб $Q=Q(y, 1 / 2)$ и числа $s_{1}<s_{2}<t_{1}<t_{2}$ такие, что $s_{1}, s_{2}, t_{1}, t_{2} \in\left[T_{0}, T_{1}\right]$, где $\left[T_{0}, T_{1}\right] \subset(0,1)$. Положим

$$
K^{-}:=Q \times\left[s_{1}, s_{2}\right], \quad K^{+}:=Q \times\left[t_{1}, t_{2}\right] .
$$

Теорема 2.5.9. Предположим, что мера $\mu=\varrho d x d t$ удовлетворяет уравнению (2.1.1), где коэфбициенты $a^{i j}, b^{i}$ удовлетворяют условиям (GP1)-(GP3) и выполнены условия (НР1)-(НР3). Тогда

$$
\sup _{K^{+}} e^{f^{-1}(|\ln (\Lambda \varrho)|)} \leqslant C\left(\int_{K^{-}} e^{-\lambda f^{-1}(|\ln (\Lambda \varrho)|)} d x d t\right)^{-1 / \lambda},
$$

где С зависит только от следующих величин:

$$
s_{1}, s_{2}, t_{1}, t_{2}, p, N, \omega_{0}, m, M, \gamma, d,\|\varrho\|_{L^{\infty}\left(\mathbb{R}^{d} \times\left[T_{0}, T_{1}\right]\right)}, \int_{T_{0}}^{T_{1}} \int_{\mathbb{R}^{d}}|b|^{p} \exp (p \psi(|b|)) \varrho d x d t .
$$

ТЕОРема 2.5.10. Предположим, что мера $\mu=\varrho d x d t$ удовлетворяет уравнению (2.1.1), где $a^{i j}, b^{i}$ удовлетворяют условиям (GP1)-(GP3) и выполнены условия (HP1)-(НР3). Зафиксируем отрезок $J=\left[\tau_{1}, \tau_{2}\right]$ из $\left(T_{0}, T_{1}\right)$. Тогда существуют такие положительные числа $c_{1}$ и $c_{2}$, что

$$
\varrho(x, t) \geqslant e^{-f\left(c_{1}|x|^{2}+c_{2}\right)}, \quad x \in \mathbb{R}^{d}, \quad t \in J .
$$

2.6. Уравнения Фоккера-Планка-Колмогорова. Здесь обсуждаются достаточные условия существования и единственности решений параболических уравнений для мер. Наше изложение следует работам [20], [19].

Будем говорить, что компактная функция $\Psi \in C^{2}\left(\mathbb{R}^{d}\right)$ невырождена, если существует последовательность таких чисел $c_{n} \rightarrow+\infty$, что множества уровня $\Psi^{-1}\left(c_{n}\right)$ являются $C^{1}$-поверхностями (на самом деле нам нужно лишь, чтобы лемма 2.1.5 была применима к областям $\left.\left\{\Psi<c_{n}\right\}\right)$. Например, если $\Psi$ выпукла, то она невырождена; то же самое верно, если $\Psi(x)=\psi_{0}\left(|x|^{2}\right)$, где $\psi_{0} \in C^{2}([0,+\infty)$ возрастает к $+\infty$.

Теорема 2.6.1. Пусть $p>d+2 u$ A, b удовлетворяют условиям

$$
\begin{gathered}
\sup _{t \in(0,1)}\left[\left\|a^{i j}(\cdot, t)\right\|_{W^{p, 1}(B)}+\left\|b^{i}(\cdot, t)\right\|_{L^{p}(B)}\right]<\infty, \\
A(x, t) \geqslant M(B) \cdot \mathrm{I} \quad \forall(x, t) \in B \times(0,1)
\end{gathered}
$$

для каждого шара $B$ при некотором $M(B)>0$. Предположим, что существуют такие невырожденная неотрицательная компактная функиия $\Psi \in$ $C^{2}\left(\mathbb{R}^{d}\right)$ и постоянная $C \geqslant 0$, что

$$
L \Psi(x, t) \leqslant C+C \Psi(x) \quad \text { n. в. в } \mathbb{R}^{d} \times(0,1) .
$$

Тогда для каждой борелевской вероятностной меры $\nu$ на $\mathbb{R}^{d}$ существует семейство $\mu=\left(\mu_{t}\right)_{t \in[0,1)}$ вероятностных мер на $\mathbb{R}^{d}$, удовлетворяющее (2.1.1) и (2.1.3), для которого функиия

$$
t \mapsto \int_{\mathbb{R}^{d}} \zeta d \mu_{t}
$$

непрерывна на $[0,1)$ для каждого $\zeta \in C_{0}^{\infty}\left(\mathbb{R}^{d}\right)$. 
Отметим, что эта теорема, установленная в [19], сильнее аналогичного результата из [18] во всех отношениях, кроме того, что здесь функция Ляпунова $\Psi$ предполагается невырожденной. Однако это довольно мягкое ограничение, которое выполняется во всех интересных случаях.

ЗАмЕчАнИЕ 2.6.2. (i) В случае, когда функции $b^{i}$ ограничены на ограниченных подмножествах $\mathbb{R}^{d} \times(0,1)$, условие невырожденности $A$ можно слегка ослабить следующим образом: достаточно иметь

$$
\inf _{(x, t) \in K \times\left[\tau_{1}, \tau_{2}\right]} \operatorname{det} A(x, t)>0
$$

для каждого отрезка $\left[\tau_{1}, \tau_{2}\right] \subset(0,1)$ и каждого компактного множества $K \subset \mathbb{R}^{d}$.

(ii) Условие (2.6.2) можно ослабить следующим образом: существуют такие компактные множества $K \subset \mathbb{R}^{d}$, что $L \Psi(x, t) \leqslant C$ п. в. в $\left(\mathbb{R}^{d} \backslash K\right) \times(0,1)$.

(iii) Для почти всех $t$ мера $\mu_{t}$ имеет плотность из класса Соболева $W_{\text {loc }}^{p, 1}\left(\mathbb{R}^{d}\right)$. Это верно для всякого решения (2.1.1) при наших локальных предположениях относительно $A$ и $b$. Значит, при этих предположениях уравнение (2.1.1) может быть записано в классической слабой форме после интегрирования по частям в слагаемом с $\partial_{x_{i}} \partial_{x_{j}} u$. Ниже мы рассмотрим более общие уравнения, решения которых не имеют такого свойства.

СлеДСтвиЕ 2.6.3. Предположим, что имеется такая постоянная $C$, что

$$
\|A(x, t)\| \leqslant C+C \ln \left(|x|^{2}+1\right), \quad(x, t) \in \mathbb{R}^{d} \times(0,1),
$$

и для каждого компактного множества $K \subset \mathbb{R}^{d}$ и каждого отрезка $\left[\tau_{1}, \tau_{2}\right]$ из $(0,1)$ мы имеем

$$
\inf _{(x, t) \in K \times\left[\tau_{1}, \tau_{2}\right]} \operatorname{det} A(x, t)>0, \quad \sup _{(x, t) \in K \times(0,1)}|b(x, t)|<\infty .
$$

Предположим также, что найдется такая постоянная $M$, что

$$
\langle b(x, t), x\rangle \leqslant M\left(1+|x|^{2}\right) \ln \left(|x|^{2}+1\right), \quad(x, t) \in \mathbb{R}^{d} \times(0,1) .
$$

Тогда для каждой борелевской вероятностной меры $\nu$ на $\mathbb{R}^{d}$ существует семейство $\left(\mu_{t}\right)_{t \in[0,1)}$ вероятностных мер на пространстве $\mathbb{R}^{d}$, удовлетворяющее (2.1.1) и (2.1.3), для которого функиия

$$
t \mapsto \int_{\mathbb{R}^{d}} \zeta d \mu_{t}
$$

непрерывна на $[0,1)$ для каждого $\zeta \in C_{0}^{\infty}\left(\mathbb{R}^{d}\right)$. Это же верно, если вместо (2.6.3) и (2.6.4) потребовать

$$
\|A(x, t)\| \leqslant C+C|x|^{2}, \quad\langle b(x, t), x\rangle \leqslant C+C|x|^{2}, \quad(x, t) \in \mathbb{R}^{d} \times(0,1) .
$$

Если же функиии $b^{i}$ и $a^{i j}$ непрерывны по $x$ для почти всякого фиксированного $t$, то это же верно без предположения, что функция $\operatorname{det} A$ строго положительна. 
СледСтвиЕ 2.6.4. Предположим, что функиии $x \mapsto a^{i j}(x, t) u x \mapsto b^{i}(x, t)$ непрерывны для каждого $t \in(0,1)$ и ограничены на ограниченных множествах в $\mathbb{R}^{d} \times(0,1)$. Кроме того, предположим, что для каждого фиксированного шара $U \subset \mathbb{R}^{d}$ функиии $x \mapsto a^{i j}(x, t), t \in(0,1)$, равностепенно непрерывны на $U$. Наконец, предположим, что существуют невырожденная неотрицательная компактная функция $\Psi \in C^{2}\left(\mathbb{R}^{d}\right)$ и постоянная $C \geqslant 0$ такие, что

$$
L \Psi(x, t) \leqslant C+C \Psi(x) .
$$

Тогда для каждой борелевской вероятностной меры д существует семейство $\mu=\left(\mu_{t}\right)_{t \in[0,1)}$ вероятностных мер на $\mathbb{R}^{d}$, удовлетворяющее (2.1.1) u (2.1.3) и такое, что функиия

$$
t \mapsto \int_{\mathbb{R}^{d}} \zeta d \mu_{t}
$$

непрерьвна на $[0,1)$ для каждого $\zeta \in C_{0}^{\infty}\left(\mathbb{R}^{d}\right)$.

Более того, если функиия $\operatorname{det} A$ отделена от нуля на компактах в множестве $\mathbb{R}^{d} \times(0,1)$, то непрерывность $b$ по $x$ не требуется.

Отметим, что эти результаты применимы к вырожденным $A$, в частности, K $A=0$.

СлЕДСтвиЕ 2.6.5. Если $\nu=\varrho_{0} d x$, то решение из теоремы 2.6 .1 удовлетворяет неравенствам (2.1.9), (2.1.10) и (2.1.11) для всякой области $\Omega \subset \mathbb{R}^{d}$, для которой обе части конечны. Если $|b(x, t)| \leqslant C \Psi(x), \Psi \in L^{1}(\nu)$, причем $\varrho_{0} \in L^{2}\left(\mathbb{R}^{d}\right)$, то выполнено и (2.4.3).

При подходящих предположениях можно построить решение, определенное для $t$ из всей прямой.

СлеДСТВИЕ 2.6.6. Предположим, что коэфбициенты $A$ и $b$ определены на всем пространстве $\mathbb{R}^{d} \times \mathbb{R}^{1}$ и удовлетворяют условию (2.6.1) на каждом ограниченном интервале вместо $(0,1)$. Предположим также, что существуют такие невырожденная неотрицательная компактная функиия $\Psi \in C^{2}\left(\mathbb{R}^{d}\right)$ и положительные числа $c_{1}$ и $c_{2}$, что

$$
L \Psi(x, t) \leqslant c_{1}-c_{2} \Psi(x) \text { для почти всех }(x, t) .
$$

Тогда существует семейство $\mu=\left(\mu_{t}\right)_{t \in \mathbb{R}^{1}}$ вероятностных мер на $\mathbb{R}^{d}$, удовлетворяющее (2.1.1), причем $\mu_{t}$ имеет плотность $\varrho(\cdot, t)$, где функция @ совместно непрерывна и положительна.

При более сильных предположениях имеются похожие теоремы единственности. Введем следующие условия.

(HU1) Существует такое $p>d+2$, что для каждого открытого шара $B \subset \mathbb{R}^{d}$ имеем

(a) $\inf _{(x, t) \in B \times[0, T]} \operatorname{det} A(x, t)>0 u \sup _{t \in[0, T], 1 \leqslant i, j \leqslant d}\left\|a^{i j}(\cdot, t)\right\|_{W^{p, 1}(B)}<\infty$,

(b) $\int_{0}^{T} \int_{B}|b(x, t)|^{p} d x d t<\infty$.

Введем следующее множество мер на $\mathbb{R}^{d} \times(0, T)$ :

$\mathscr{M}_{\mathrm{par}}^{A, b, \nu}:=\left\{\mu \mid \mu(d x, d t)=\mu_{t}(d x) d t, \mu_{t} \in \mathscr{P}\left(\mathbb{R}^{d}\right) \forall t \in(0, T)\right.$ и $\mu$ удовлетворяет (2.1.1), (2.1.3), где $|b| \in L^{1}(B \times(0, T), \mu)$ для каждого шара $\left.B \subset \mathbb{R}^{d}\right\}$. 
ТЕОРема 2.6.7. Предположим, что выполнено (HU1). Кроме того, предположим, что выполнено следующее условие:

(HU2) каждая функиия $a^{i j}$ гёльдерова по $t \in[0, T]$ локально равномерно относительно $x \in \mathbb{R}^{d}$.

Пусть множество $\mathscr{K} \subset \mathscr{M}_{\mathrm{par}}^{A, b, \nu}$ таково, что $\mathscr{K}$ выпукло и для всех $\mu \in \mathscr{K}$

$$
(1-L)\left(C_{0}^{\infty}\left(\mathbb{R}^{d} \times[0, T)\right)\right) \text { плотно в } L^{1}\left(\mathbb{R}^{d} \times(0, T), \mu\right) .
$$

Тогда \# $\mathscr{K} \leqslant 1$.

Теперь дадим конструктивные условия на коэффициенты, позволяющие использовать приведенный результат.

Зададим логарифмический градиент $\beta_{\mu}=\left(\beta_{\mu}^{1}, \ldots, \beta_{\mu}^{d}\right)$ меры $\mu$ относительно метрики, порожденной $A$, следующим образом:

$$
\beta_{\mu}^{i}:=\sum_{j=1}^{d}\left(\partial_{x_{j}} a^{i j}+a^{i j} \varrho^{-1} \partial_{x_{j}} \varrho\right), \quad i=1, \ldots, d .
$$

ПРЕДЛОЖЕНИЕ 2.6.8. Предположим, что выполнены (HU1) и (HU2) и определим $\mathscr{K}$ как множество всех мер $\mu \in \mathscr{M}_{\mathrm{par}}^{A, b, \nu}$, удовлетворяющих следующим трем условиям для всех $1 \leqslant i, j \leqslant d$ :

(i) $\partial_{x_{j}} a^{i j} \in L^{1}(B \times(0, T), \mu)$ для всех открытых шаров $B \subset \mathbb{R}^{d}$;

(ii) $a^{i j} \in L^{1}\left(\mathbb{R}^{d} \times(0, T), \mu\right)$;

(iii) $b^{i}-\beta_{\mu}^{i} \in L^{1}\left(\mathbb{R}^{d} \times(0, T), \mu\right)$.

Тогда \# $\mathscr{K} \leqslant 1$.

ПреДЛОЖЕНИЕ 2.6.9. Пусть выполненъ (HU1) $u$ (HU2), а функиия $V \in$ $C^{1,2}\left(\mathbb{R}^{d} \times[0, T]\right)$ такова, что $\lim _{|x| \rightarrow \infty} V(x, t)=+\infty$ равномерно по $t \in[0, T]$. Пусть $\mathscr{K}-$ множество всех мер $\mu \in \mathscr{M}_{\mathrm{par}}^{A, b, \nu}$, удовлетворяющих условию (i) в предложении 2.6 .8 и таких, что для некоторого $\alpha_{0}=\alpha_{0}(\mu) \in(0, \infty)$ выполнено неравенство

$$
L_{A, 2 \beta_{\mu}-b} V-\partial_{t} V \leqslant \alpha_{0} V \text {. }
$$

Тогда \# $\mathscr{K}=1$.

Теорема 2.6.10. Предположим, что выполнены (HU1) и (HU2). Предположим также, что выполнены следующие глобальные условия на $A, b$ u

(iv) мера $\nu$ имеет конечную энтропию, m.е. $\nu=\varrho_{0} d x u \varrho_{0} \ln \varrho_{0} \in L^{1}\left(\mathbb{R}^{d}\right)$;

(v) существует такое $\varepsilon \in(0, \infty)$, что

$$
\varepsilon \mathrm{I} \leqslant A(x, t) \leqslant \varepsilon^{-1} \mathrm{I} \quad \text { для всех }(x, t) \in \mathbb{R}^{d} \times[0, T]
$$

(vi) существует такое $\Lambda \in(0, \infty)$, что для всех $x, y \in \mathbb{R}^{d}$

$$
\sup \left\{\left|a^{i j}(x, t)-a^{i j}(y, t)\right|: t \in[0, T], 1 \leqslant i \leqslant j \leqslant d\right\} \leqslant \Lambda|x-y| ;
$$

(vii) существует такое $c \in(0, \infty)$, что для всех $(x, t) \in \mathbb{R}^{d} \times[0, T]$

$$
\langle b(x, t), x\rangle \leqslant c\left(1+|x|^{2}\right),
$$


и либо для некоторого $k \in \mathbb{N}$ мы имеем

$$
|b(x, t)| \leqslant c\left(1+|x|^{2 k}\right) \quad u \quad \int_{\mathbb{R}^{d}}|x|^{2 k} \nu(d x)<\infty,
$$

либо найдутся такие числа $\alpha, \gamma, \delta, c, k \in(0, \infty)$, что для всех $(x, t) \in \mathbb{R}^{d} \times[0, T]$ верно неравенство

$$
\langle b(x, t), x\rangle \leqslant \gamma-\left(2 \varepsilon^{-1} c k+\delta\right)|x|^{2 k}
$$

с тем же в, что и в (v), причем

$$
|b(x, t)| \leqslant \alpha \exp \left(\frac{c}{2}|x|^{2 k}\right), \quad \int_{\mathbb{R}^{d}} \exp \left(\frac{c}{2}|x|^{2 k}\right) \nu(d x)<\infty .
$$

Тогда существует единственное семейство $\left\{\mu_{t}, t \in(0, T]\right\}$ вероятностных мер на $\mathbb{R}^{d}$, удовлетворяющее (2.1.1), (2.1.3).

Рассмотренные уравнения для мер по указанной выше терминологии представляют собой уравнения Фоккера-Планка. Мы не обсуждаем здесь тесно с ними связанные уравнения Колмогорова для функций (см., например, [130]-[132]). Наконец, отметим, что в [133] похожие теоремы существования недавно получены для нелинейных уравнений для мер.

\section{3. Бесконечномерный случай}

3.1. Уравнения для мер на бесконечномерных пространствах. Проблемы, рассмотренные в предыдущих двух разделах, возникают также и в бесконечномерном случае; как было отмечено во введении, бесконечномерный случай даже послужил одним из источников обращения к таким проблемам. Мы кратко обсудим эллиптические уравнения, поскольку подробное рассмотрение всей этой области заслуживает отдельного обзора. Более того, наше обсуждение будет ограничено вопросами, непосредственно связанными с конечномерным случаем. Прежде всего следует отметить, что многие основные объекты, рассмотренные выше, такие как стохастические уравнения, переходные вероятности, стационарные распределения, уравнения Фоккера-Планка и Колмогорова, определены на весьма общих пространствах и не имеют какой-либо конечномерной специфики. Однако в бесконечномерном случае возникают новые явления. Частично это связано с отсутствием точных аналогов меры Лебега, но имеются также и другие причины. Одним из исходных пунктов опять является марковский процесс с пространством состояний $X$, для которого переходная полугруппа $\left(T_{t}\right)_{t \geqslant 0}$ определена на подходящем функциональном пространстве $\mathscr{F}$ (например, $C_{b}(X)$ или $\left.L^{p}(\mu)\right)$. Если этот процесс имеет инвариантную вероятностную меру $\mu$ на $X$, т. е.

$$
\int_{X} f(x) \mu(d x)=\int_{X} T_{t} f(x) \mu(d x), \quad f \in \mathscr{F},
$$

то при широких предположениях $\left(T_{t}\right)_{t \geqslant 0}$ продолжается до сильно непрерывной полугруппы в $L^{1}(\mu)$, которая имеет генератор $L$ на некоторой области $\mathscr{D}$, и $\mu$ удовлетворяет уравнению

$$
L^{*} \mu=0
$$


в том смысле, что интеграл от $L f$ равен нулю для всех $f \in \mathscr{D}$. В типичных случаях имеется меньший класс $\mathscr{D}_{0} \subset \mathscr{D}$ функций, для которых выражение $L f$ определено явно, причем вне каких-либо связей с полугруппой и областью определения ее генератора. Тогда можно рассмотреть уравнение (3.1.1) как тождество

$$
\int_{X} L f(x) \mu(d x)=0, \quad f \in \mathscr{D}_{0} .
$$

Следовательно, возникают вопросы о связи между уравнением (3.1.2) и инвариантностью меры $\mu$ относительно полугруппы, а также вопрос о свойствах решений уравнения (3.1.2), т. е. те же самые вопросы, которые мы обсуждали в конечномерном случае. Например, пусть $\mu$ - инвариантная мера диффузионного процесса в $l^{2}$ или в пространстве $\mathbb{R}^{\infty}$ всех вещественных последовательностей, заданная стохастическим дифференциальным уравнением

$$
d \xi_{t}=\sqrt{2} d W_{t}+b\left(\xi_{t}\right) d t
$$

где $W_{t}$ - винеровский процесс (в подходящем смысле, см. [134; гл. 7]) и $b-$ борелевское векторное поле. Тогда можно взять в качестве $\mathscr{D}_{0}$ класс $\mathscr{F} \mathscr{C}_{b}^{\infty}$ всех функций вида

$$
f\left(l_{1}(x), \ldots, l_{n}(x)\right),
$$

где $f \in C_{b}\left(\mathbb{R}^{n}\right)$ и $l_{1}, \ldots, l_{n}$ - непрерывные линейные функционалы (например, просто функции от $\left.x_{1}, \ldots, x_{n}\right)$. Тогда $L$ имеет вид

$$
L f=\operatorname{trace} f^{\prime \prime}+\left\langle f^{\prime}, b\right\rangle .
$$

Однако, как мы увидим ниже в конкретных примерах, в бесконечномерном случае часто желательно иметь более широкую постановку, в которой $b$ нельзя интерпретировать как векторное поле со значениями в исходном пространстве (например, если мы имеем дело с $l^{2}$, может случиться, что $v$ принимает значения в $\left.\mathbb{R}^{\infty}\right)$. По этой причине используется следующая более общая схема.

Предположим, что $X$ - локально выпуклое пространство с сопряженным $X^{*}$ и $H \subset X$ - плотное сепарабельное гильбертово пространство, причем оператор вложения непрерывен. Скалярное произведение в $H$ обозначим через $(u, v)_{H}$. Это вложение задает вложение $j_{H}: X^{*} \rightarrow H$, поскольку для каждого $l \in X^{*}$ есть единственный элемент $j_{H}(l) \in H$, для которого

$$
\langle l, h\rangle=\left(j_{H}(l), h\right)_{H} \quad \forall h \in H .
$$

Предположим также, что семейство $\left\{l_{i}\right\} \subset X^{*}$ таково, что векторы $e_{n}:=j_{H}\left(l_{n}\right)$ образуют ортонормированный базис в $H$. Обозначим через $\mathscr{F}_{b}^{\infty}\left(\left\{l_{i}\right\}\right)$ класс всех функций вида (3.1.4). Предположим также, что даны борелевские функции $a^{i j}, b^{i}$ на $X$. Бесконечная матрица с элементами $a^{i j}(x)$ будет обозначаться через $A(x)$ и набор скалярных функций $b^{i}$ будет обозначаться через $b$, хотя мы не предполагаем, что $A(x)$ и $b(x)$ соответствуют каким-либо оператору или вектору в $X$. Теперь рассмотрим эллиптический оператор

$$
L_{A, b} f(x)=\sum_{i, j} a^{i j}(x) \partial_{e_{i}} \partial_{e_{j}} f(x)+\sum_{i} b^{i}(x) \partial_{e_{i}} f(x) .
$$


Будем говорить, что радоновская мера $\mu$ на $X$ удовлетворяет уравнению (3.1.1) относительно класса $\mathscr{F} \mathscr{C}_{b}^{\infty}\left(\left\{l_{i}\right\}\right)$, если $a^{i j}, b^{i} \in L^{1}(\mu)$ для всех $i, j$ и (3.1.2) выполнено для $\mathscr{D}_{0}=\mathscr{F}_{b}^{\infty}\left(\left\{l_{i}\right\}\right)$. Параболические операторы и уравнения определяются похожим образом в полной аналогии с конечномерным случаем.

Бесконечномерные уравнения для мер пока изучены гораздо менее по сравнению с конечномерным случаем. Имеются достаточные условия существования и иногда единственности решений, но немного известно об их свойствах и связях между инфинитезимальной инвариантностью и собственной инвариантностью относительно ассоциированных полугрупп (само существование таких полугрупп также менее изучено). Существует обширная литература по стационарным распределениям бесконечномерных диффузий (см., например, книгу [89]), особенно связанных со стохастическими дифференциальными уравнениями с частными производными (см. [132], [135]-[148]), бесконечными градиентными системами, гиббсовскими мерами и стохастическим квантованием (см. [54], [149]-[155]); в этих работах можно найти многочисленные дополнительные ссылки. Стандартные методы доказательства существования стационарных распределений основаны на теореме Прохорова и функциях Ляпунова в сочетании с априорными оценками (см., например, [156]-[158]) или на сходимости переходных вероятностей (что в свою очередь привлекает различные условия диссипативности и функции Ляпунова). Такая же техника используется для доказательства существования решений эллиптических или параболических уравнений. Вообще говоря, результаты о существовании для эллиптических уравнений могут быть получены при более широких предположениях относительно коэффициентов, поскольку они не предполагают существования соответствующих диффузий (последнее обычно более ограничительно); однако имеются случаи, когда нет прямых доказательств разрешимости эллиптического уравнения для мер и приходится строить процесс и анализировать его переходную полугруппу.

Для решений эллиптических и параболических уравнений для мер на бесконечномерных пространствах можно рассматривать различные свойства их конечномерных проекций, их взаимную абсолютную непрерывность или сингулярность, а также свойства по направлениям, такие как непрерывность или дифференцируемость. Напомним, что радоновская мера $\mu$ на локально выпуклом пространстве $X$ непрерывна вдоль вектора $h \in X$, если $\lim _{t \rightarrow 0}\left\|\mu_{t h}-\mu\right\|=0$, где $\mu_{t h}(B):=\mu(B+t h)$ для каждого борелевского множества $B$. Если для каждого борелевского множества $B$ функция $t \mapsto \mu(B+t h)$ дифференцируема, то $\mu$ называется дифференцируемой вдоль $h$ по Фомину. Это равносильно существованию такой функции $\beta_{h}^{\mu} \in L^{1}(\mu)$, называемой логарифмической производной меры $\mu$ вдоль $h$, что следующая формула интегрирования по частям верна для всех функций $f \in \mathscr{F} \mathscr{C}_{b}^{\infty}(X)$ :

$$
\int_{X} \partial_{h} f(x) \mu(d x)=-\int_{X} f(x) \beta_{h}^{\mu}(x) \mu(d x) .
$$

Если $X=\mathbb{R}^{d}$, то мера $\mu$ дифференцируема по Фомину вдоль всех векторов в точности тогда, когда она имеет плотность $\varrho \in W^{1,1}\left(\mathbb{R}^{d}\right)$; в этом случае $\beta_{h}^{\mu}=\langle\nabla \varrho, h\rangle$. В бесконечномерном случае лишь нулевая мера дифференцируема вдоль всех векторов. Подробное изложение теории дифференцируемых 
мер дано в [159], [26]. Имеется также разумный бесконечномерный аналог логарифмического градиента $\nabla \varrho / \varrho$, хотя ни $\varrho$, ни $\nabla \varrho$ не существуют по отдельности. А именно, предположим, что дано непрерывно и плотно вложенное гильбертово пространство $H \subset X$, порождающее вложение $j: X^{*} \rightarrow X$ как объяснено выше. Если существует такое $\mu$-измеримое отображение $\beta_{H}: X \rightarrow X$, что для каждого $l \in X^{*}$ мера $\mu$ дифференцируема вдоль $j(l)$ и

$$
\beta_{j(h)}^{\mu}=\left\langle l, \beta_{H}\right\rangle
$$

то $\beta_{H}$ называется логарифмическим градиентом меры $\mu$, ассоциированным с $H$. Однако может случиться, что мера $\mu$ дифференцируема вдоль всех векторов $j(l)$, где $l \in X^{*}$, но нет логарифмического градиента. Об этом объекте см. [26; гл. 7].

Пусть векторы $\left\{l_{i}\right\} \subset X^{*}$ таковы, что для векторов $e_{i}:=j\left(l_{i}\right)$ имеем $l_{j}\left(e_{i}\right)=\delta_{i j}$. Предположим дополнительно, что функционалы $l_{i}$ разделяют точки в $X$. Тогда при довольно мягких предположениях относительно $X$ (см. [134; гл. 7]) существует процесс $W_{t}$ в $X$, называемый винеровским процессом в $X$, ассоциированным с $H$, для которого скалярные процессы $\left\langle l_{i}, W_{t}\right\rangle$ являются независимыми винеровскими. Известно (см. [160], [26; гл. 12]), что при широких предположениях для данной вероятностной меры $\mu$, обладающей логарифмическим градиентом $\beta_{H}$, существует такой диффузионный процесс в $X$, подчиняющийся стохастическому дифференциальному уравнению (3.1.3) при $b=\beta_{H}$, что $\mu$ - его инвариантная мера и соответствующая переходная полугруппа $\left(T_{t}\right)_{t \geqslant 0}$ симметрична на $L^{2}(\mu)$; ее генератор задан формулой

$$
L f=\sum_{i=1}^{\infty}\left[\partial_{e_{i}}^{2} f+\left\langle l_{i}, \beta_{H}\right\rangle \partial_{e_{i}} f\right]
$$

на функциях $f \in \mathscr{F} \mathscr{C}_{b}^{\infty}\left(\left\{l_{i}\right\}\right)$. Эффективный метод построения более общих процессов основан на возмущении сноса $\beta_{H}$.

Аналогично конечномерному случаю симметричность оператора $L_{\mathrm{I}, b}$ равносильна тому, что $b$ имеет в некотором смысле градиентный вид. Точный результат состоит в следующем.

ПРЕДЛОЖЕНИЕ 3.1.1. Пусть $b^{i} \in L^{2}(\mu)$. Onератор

$$
L f=\sum_{i=1}^{\infty}\left[\partial_{e_{i}}^{2} f+b^{i} \partial_{e_{i}} f\right]
$$

симметричен на $\mathscr{F}_{b}^{\infty}\left(\left\{l_{i}\right\}\right) \subset L^{2}(\mu)$ в точности тогда, когда мера $\mu$ дифферениируема вдоль каждого вектора $e_{n}=j\left(l_{n}\right)$ и $\beta_{e_{n}}^{\mu}=b^{n} \mu-n . в$.

ДокАЗАтЕЛЬство. Если $\beta_{e_{n}}^{\mu}$ существует, то

$$
\int_{X} g(x) \partial_{e_{n}}^{2} f(x) \mu(d x)=-\int_{X}\left[\partial_{e_{n}} g(x) \partial_{e_{n}} f(x)+g(x) \partial_{e_{n}} f(x) \beta_{e_{n}}^{\mu}(x)\right] \mu(d x),
$$

откуда получаем равенство интегралов $g L f$ и $f L g$ для всех функций $f, g \in$ $\mathscr{F} \mathscr{C}_{b}^{\infty}\left(\left\{l_{i}\right\}\right)$. Обратно, предположим, что $L$ симметричен на $\mathscr{F} \mathscr{C}_{b}^{\infty}\left(\left\{l_{i}\right\}\right)$. Пусть 
$f=\exp (i l), g=\exp \left(i t l_{n}\right)$, где $l-$ конечная линейная комбинация функционалов $l_{i}$. Положим $\langle l, b\rangle:=\sum_{k=1}^{\infty} l\left(e_{k}\right) b^{k}$, где сумма конечна, поскольку лишь конечное число величин $l\left(e_{k}\right)$ отличны от нуля. Тогда

$$
L f=-\sum_{k=1}^{\infty} l\left(e_{k}\right)^{2} e^{i l}+i\langle l, b\rangle e^{i l}, \quad L g=-t^{2} e^{i t l_{n}}+i t b^{n} e^{i t l_{n}},
$$

откуда по симметричности $L$ получаем равенство

$$
\int_{X} e^{i l+i t l_{n}}\left[-\sum_{k=1}^{\infty} l\left(e_{k}\right)^{2}+i\langle l, b\rangle\right] \mu(d x)=\int_{X} e^{i l+i t l_{n}}\left[-t^{2}+i t b^{n}\right] \mu(d x) .
$$

Полагая $\xi:=l+t l_{n}$ и заменяя $l$ на $\xi-t l_{n}$, можно записать это следующим образом:

$$
\int_{X} e^{i \xi}\left[-\sum_{k=1}^{\infty} \xi\left(e_{k}\right)^{2}+i\langle\xi, b\rangle\right] \mu(d x)=2 \int_{X} e^{i \xi}\left[-t \xi\left(e_{n}\right)+i t b^{n}\right] \mu(d x) .
$$

Поскольку левая часть не зависит от $t$, то правая часть должна быть нулем. Следовательно, мы получаем

$$
-i \xi\left(e_{n}\right) \int_{X} e^{i \xi} \mu(d x)=\int_{X} e^{i \xi} b^{n} \mu(d x)
$$

для всякого функционала $\xi$, являющегося конечной линейной комбинацией $l_{i}$. Поскольку $\mu$ - радоновская мера и $\left\{l_{i}\right\}$ разделяет точки в $X$, то это же самое верно для всякого $\xi \in X^{*}$. Это тождество показывает, что мера $\mu$ дифференцируема вдоль $e_{n}$ и $\beta_{e_{n}}^{\mu}=b^{n}$ (см. [26; теорема 3.6.7]). Заметим, что без предположения, что $\left\{l_{i}\right\}$ разделяет точки в $X$, мы могли бы получить то же самое утверждение для $\mu$ на $\sigma$-алгебре, порожденной $\left\{l_{i}\right\}$. Предложение доказано.

Случай непостоянного $A$ похожим образом изучен в [161].

Случай сносов градиентного типа (это понятие в свою очередь допускает различные интерпретации в бесконечной размерности) имеет глубокие и интересные связи с изучением гиббсовских мер, т. е. мер с заданными условными распределениями. Например, для широкого класса моделей построение гиббсовских мер равносильно построению мер с заданными логарифмическими градиентами; последняя задача изучается в [149], [150], [159], [26], [13]-[16]; в частности, метод восстановления мер с данным логарифмическим градиентом посредством функций Ляпунова был предложен А. И. Кирилловым. Для общих эллиптических уравнений для мер этот метод был развит в [4], [10], [24]. Задача единственности для эллиптических уравнений относительно мер становится особенно трудной в бесконечной размерности. Имеются простые примеры, в которых нет единственности даже для $A=I$ и сносов $b$, являющихся ограниченными линейными операторами в гильбертовом пространстве (см. [4], [26; $\S 7.6])$. В рамках теории гиббсовских мер такие примеры возникают в случаях фазовых переходов, т. е. различных гиббсовских распределений с равными условными распределениями. Относительно существования и единственности решений параболических уравнений см. [162], [130], [163]. 
Приведем ряд характерных примеров, в которых описанная выше схема применяется для исследования стохастических уравнений с частными производными типа стохастических уравнений пористых сред, диффузионной реакции, Бюргерса и Навье-Стокса. Точные формулировки и дополнительные ссылки можно найти в [10], [164]. Общий план рассмотрения подобных уравнений таков. Первичный объект - нелинейное уравнение с частными производными вида

$$
\frac{\partial \xi(u, t)}{\partial t}=\Delta[\Psi(\xi)](u, t)+\Phi(\xi)(u, t)
$$

где $\Psi$ и $\Phi$ - некоторые функции на прямой, например, многочлены. Анализ этого уравнения оказывается обычно весьма сложным (как, например, уравнение Навье-Стокса). Однако выясняется, что добавление случайного шума в правую часть приводит к существенным упрощениям (возможно даже, что стохастическое уравнение имеет не меньший физический смысл). Такое стохастическое уравнение с частными производными эвристически записывается как

$$
d \xi_{t}=\sqrt{2} d W_{t}+\left(\Delta\left[\Psi\left(\xi_{t}\right)\right]+\Phi\left(\xi_{t}\right)\right) d t
$$

Однако строгая интерпретация в случае нелинейных функций $\Psi$ и $\Phi$ не очевидна. Один из возможных подходов к этой проблеме состоит в том, чтобы рассмотреть ассоциированный бесконечномерный эллиптический оператор $L$ на подходящей области, найти инфинитезимально инвариантную меру $\mu$ для $L$, построить марковскую полугруппу на $L^{2}(\mu)$ с $\mu$ в качестве инвариантной меры так, что генератор полугруппы продолжает $L$, и, наконец, построить марковский процесс, дающий решение мартингальной задачи, соответствующей данному оператору. Можно также пытаться решать параболическое уравнение для переходных вероятностей ожидаемого процесса. Случай $\Psi(s)=s^{m}$ и $\Phi=0$ соответствует уравнению пористых сред, случай $\Psi(s)=s$ и $\Phi(s)=s^{m}$ - уравнению диффузионной реакции.

Пусть $D \subset \mathbb{R}^{d}$ - ограниченная открытая область с гладкой границей, $\left\{e_{n}\right\}-$ ортонормированный базис в $L^{2}(D)$, образованный собственными функциями лапласиана $\Delta$ с граничными условиями Дирихле, $\lambda_{1}$ - наименьшее по модулю собственное число. Пусть $\Psi$ - такая $C^{1}$-функция с $\Psi(0)=0$, что для некоторых положительных чисел $\kappa_{0}, C_{0}, \kappa_{1}$ и $r \geqslant 1$ имеем

$$
\kappa_{0}|s|^{r-1} \leqslant \Psi^{\prime}(s) \leqslant C_{0}+\kappa_{1}|s|^{r-1} \quad \text { при всех } s \in \mathbb{R}^{1} \text {, }
$$

и пусть $\Phi$ - непрерывная функция, удовлетворяющая следующему условию:

$$
|\Phi(s)| \leqslant C+\delta|s|^{r}
$$

где $0<\delta<4 \kappa_{0} \lambda_{1}(r+1)^{-2}$ и $C$ - постоянная. Например, достаточно, чтобы $|\Phi(s)| \leqslant \kappa_{2}+\kappa_{3}|s|^{q}$, где $q \in(0, r), \kappa_{2}, \kappa_{3} \in(0,+\infty)$. Нас интересует существование инфинитезимально инвариантных мер для бесконечномерного эллиптического оператора $L$, который неформально задан посредством

$$
L f:=\Delta_{Q} f+\langle b, \nabla f\rangle, \quad b(x)=\Delta \Psi(x)+\Phi(x)
$$


на гладких цилиндрических функциях, определенных на $X:=L^{2}(D)$ или на отрицательном соболевском пространстве $H^{2,-1}(D)$. Строгая интерпретация такова. Положим

$$
b^{i}(x):=\int_{D}\left[\Psi(x(u)) \Delta e_{i}(u)+\Phi(x(u)) e_{i}(u)\right] d u, \quad x \in L^{r}(D) .
$$

Пусть $q_{i}>0$ таковы, что $S:=\sum_{i=1}^{\infty} q_{i}<\infty$. Оператор

$$
L f:=\sum_{i=1}^{\infty}\left[q_{i} \partial_{e_{i}}^{2} f+b^{i} \partial_{e_{i}} f\right]
$$

где $\partial_{e_{i}}$ обозначает частную производную вдоль вектора $e_{i}$, корректно определен на классе $\mathscr{F} \mathscr{C}_{b}^{\infty}\left(\left\{l_{i}\right\}\right)$, где $l_{i}(x)=\left(x, e_{i}\right)_{2}$ и $(x, y)_{2}$ - скалярное произведение в $L^{2}(D)$. Часть второго порядка в $L$ можно рассматривать как $\operatorname{trace}\left(Q D^{2} f\right)$, где $Q$ - оператор на $X$, определенный посредством $Q e_{i}=q_{i} e_{i}$. Оператор $Q$ является ковариацией винеровского процесса $W_{t}$ в указанном стохастическом уравнении. Небольшой нюанс состоит в том, что функции $b^{i}$, а потому и $L f$, заданы не на всем $X$, а лишь на $L^{r}(D)$.

Теорема 3.1.2. При сделанных выше предположениях существует борелевская вероятностная мера $\mu$ на $L^{r}(D)$, являющаяся инфинитезимально инвариантной для $L$.

Например, если $\Psi(t)=t^{r}$, где $r$ - нечетное число, то можно взять в качестве $\Phi$ любой многочлен степени $r$ с достаточно малым старшим коэффициентом (малость которого зависит от $\lambda_{1}$, в частности, можно взять $\Phi(x)=x^{r}$ при условии, что $\lambda_{1}$ достаточно велико). Аналогично рассматривается параболическое уравнение с зависящим от времени сносом $b$, формально заданным посредством $b(x, t)(u)=\Delta_{u}[\Psi(x(u), t)]+\Phi(x(u), t)$, где $\Psi$ и $\Phi-$ вещественные функции на $\mathbb{R}^{1} \times[0,1]$. Положим

$$
b^{i}(x, t):=\int_{D}\left[\Psi(x(u), t) \Delta e_{i}(u)+\Phi(x(u), t) e_{i}(u)\right] d u, \quad x \in L^{r}(D) .
$$

Соответствующий параболический оператор $L$ задан посредством

$$
L f=\partial_{t} f+\sum_{i=1}^{\infty} q_{i} \partial_{e_{i}}^{2} f+\sum_{i=1}^{\infty} b^{i} \partial_{e_{i}} f .
$$

Предположим, что $\Psi$ и $\Phi$ - непрерывные функции, $\Psi$ имеет непрерывную частную производную $\partial_{s} \Psi(s, t)$, причем

$$
\kappa_{0}|s|^{r-1} \leqslant \partial_{s} \Psi(s, t) \leqslant C_{1}+\kappa_{1}|s|^{r-1}, \quad|\Phi(s, t)| \leqslant C_{2}+\kappa_{2}|s|^{r},
$$

где $\kappa_{0}, \kappa_{1}, \kappa_{2}, C_{1}, C_{2} \in(0,+\infty)$ - некоторые постоянные и $r \geqslant 1$. При этих условиях существует вероятностная мера $\mu$ на $L^{r}(D) \times[0,1)$, удовлетворяющая параболическому уравнению $L^{*} \mu=0$ с подходящим начальным условием.

Стохастическое уравнение типа Навье-Стокса рассматривается в пространстве $X$ всех $\mathbb{R}^{d}$-значных отображений $\xi=\left(\xi^{1}, \ldots, \xi^{d}\right)$, для которых $\xi^{j} \in W_{0}^{2,1}(D)$ 
и $\operatorname{div} \xi=0$. Пространство $X$ наделяется гильбертовой нормой $\|\xi\|_{0}$, определенной посредством $\|\xi\|_{0}^{2}:=\sum_{j=1}^{d}\left\|\nabla \xi^{j}\right\|_{L^{2}}^{2}$. Уравнение формально записывается в виде

$$
d \xi(x, t)=\sqrt{2} d W(x, t)+\left[\Delta_{x} \xi(x, t)-\sum_{j=1}^{d} \xi^{j}(x, t) \partial_{x_{j}} \xi(x, t)+F(x, \xi(x, t), t)\right] d t
$$

где $W$ - подходящий винеровский процесс в $X$ и $F: D \times \mathbb{R}^{d} \times(0,1) \rightarrow \mathbb{R}^{d}-$ ограниченное непрерывное отображение. Так как лапласиан $\Delta$ определен не на всем $X$, то это уравнение требует некоторой интерпретации. Наш подход предлагает следующую процедуру. Пусть $\left\{\eta_{n}\right\}$ - ортонормированный базис в замыкании $X$ в $L^{2}\left(D, \mathbb{R}^{d}\right)$, образованный собственными функциями $\Delta$, причем $\eta_{n} \in X$. Введем функции

$$
b^{n}(\xi, t):=\left(\xi, \Delta \eta_{n}\right)_{2}-\sum_{j=1}^{d}\left(\partial_{x_{j}} \xi, \xi^{j} \eta_{n}\right)_{2}+\left(F(\cdot, \xi(\cdot), t), \eta_{n}\right)_{2}
$$

Эти функции определены уже на всем $X$. Легко проверить, что они непрерывны на шарах из $X$ относительно топологии из $L^{2}\left(D, \mathbb{R}^{d}\right)$. Выбрав подходящий винеровский процесс, приходим к параболическому оператору

$$
L f(\xi, t)=\partial_{t} f(\xi, t)+\sum_{n=1}^{\infty} \alpha_{n} \partial_{\eta_{n}}^{2} f(\xi, t)+\sum_{n=1}^{\infty} b^{n}(\xi, t) \partial_{\eta_{n}} f(\xi, t) .
$$

Здесь также есть вероятностная мера $\mu$ на $X \times[0,1)$, удовлетворяющая параболическому уравнению $L^{*} \mu=0$ с любым начальным распределением $\mu_{0}$, для которого $\|\xi\|_{0}^{2}\|\xi\|_{2}^{k} \in L^{1}\left(\mu_{0}\right)$ при всех $k$. Если $F$ не зависит от $t$, то аналогично рассматривается эллиптическое уравнение и устанавливается существование инфинитезимально инвариантных мер стохастического уравнения Навье-Стокса.

3.2. Свойства решений. Результаты раздела 1 дают некоторые свойства конечномерных проекций меры $\mu$, удовлетворяющей бесконечномерному уравнению, порожденных отображениями $P_{n} x=\left(l_{1}(x), \ldots, l_{n}(x)\right)$. Будем предполагать, что даны вложение $H \subset X$, порождающее вложение $j: X^{*} \rightarrow H$, причем функционалы $l_{i}$ таковы, что векторы $e_{i}:=j\left(l_{i}\right)$ обладают свойством $l_{i}\left(e_{j}\right)=\delta_{i j}$. Пусть $\mu-$ вероятностная мера, удовлетворяющая уравнению $L_{A, b}^{*} \mu=0$ с $A=\left(a^{i j}\right)_{i, j \geqslant 1}$ и $b=\left(b^{i}\right)_{i \geqslant 1}$ в объясненном выше смысле.

Обозначим через $\mathrm{E}_{n}$ условное математическое ожидание относительно $\sigma$-алгебры, порожденной $P_{n}$, и положим

$$
\sigma_{n}^{i j}:=\mathrm{E}_{n} \sigma^{i j}, \quad b_{n}^{i}:=\mathrm{E}_{n} b^{i} .
$$

Рассмотрим эллиптический оператор

$$
L_{n} f:=\sum_{i, j=1}^{n} a_{n}^{i j}(x) \partial_{x_{i}} \partial_{x_{j}} f(x)+\sum_{i=1}^{n} b_{n}^{i}(x) \partial_{x_{i}} f(x)
$$


на $\mathbb{R}^{n}$. Для мер $\mu_{n}:=\mu \circ P_{n}^{-1}, \mu \circ P_{n}^{-1}(B):=\mu\left(P_{n}^{-1}(B)\right)$, на $\mathbb{R}^{n}$ получим

$$
L_{n}^{*} \mu_{n}=0 \text {. }
$$

В самом деле, если $f(x)=f\left(l_{1}(x), \ldots, l_{n}(x)\right)$, то

$$
\begin{aligned}
\int_{\mathbb{R}^{n}} L_{n} \varphi(y) \mu_{n}(d y)= & \int_{X}\left[\sum_{i, j \leqslant n} a_{n}^{i j}(x) \partial_{e_{i}} \partial_{e_{j}} \varphi\left(l_{1}(x), \ldots, l_{n}(x)\right)\right. \\
& \left.+\sum_{i \leqslant n} b_{n}^{i}(x) \partial_{e_{i}} \varphi\left(l_{1}(x), \ldots, l_{n}(x)\right)\right] \mu(d x) \\
= & \int_{X}\left[\sum_{i, j \leqslant n} a^{i j}(x) \partial_{e_{i}} \partial_{e_{j}} \varphi\left(l_{1}(x), \ldots, l_{n}(x)\right)\right. \\
& \left.+\sum_{i \leqslant n} b^{i}(x) \partial_{e_{i}} \varphi\left(l_{1}(x), \ldots, l_{n}(x)\right)\right] \mu(d x)=0 .
\end{aligned}
$$

ПРимеР 3.2.1. Пусть функции $a^{i j}$ постоянны и матрицы $\left(a^{i j}\right)_{i, j \leqslant n}$ положительны, причем $b^{i} \in L^{p}(\mu)$ для всех $p<\infty$. Тогда $\mu_{n}$ имеет ограниченную непрерывную плотность $\varrho_{n}$ класса $W^{p, 1}\left(\mathbb{R}^{n}\right)$ для всех $n$.

Если имеется лишь включение $b^{i} \in L^{2}(\mu)$, то $\mu_{n}$ обладает плотностью $\varrho_{n}$ класса $W^{1,1}\left(\mathbb{R}^{n}\right)$ и $\left|\nabla \varrho_{n} / \varrho_{n}\right|^{2} \in L^{2}\left(\mu_{n}\right)$.

Если же $\exp \left(c_{i}\left|b^{i}\right|\right) \in L^{1}(\mu)$ для некоторых $c_{i}>0$, то непрерывная плотность $\varrho_{n}$ положительна.

Другие применения результатов первых двух разделов к конечномерным проекциям решений бесконечномерных уравнений для мер даны в [23]. Для некоторых специальных уравнений имеются другие результаты, например, некоторые равномерные локальные оценки мер шаров и плотностей, см. [165], [166], [148]. Однако остается открытым такой вопрос. Пусть вероятностная мера $\mu$ на сепарабельном гильбертовом пространстве удовлетворяет уравнению $L_{I, b}^{*}=\mu=0$ с непрерывным или даже локально липшицевым сносом $b$; положительна ли она на всех шарах ненулевого радиуса? В ряде специальных случаев известны положительные результаты; например, так будет, если мера $\mu$ эквивалентна какой-либо мере, обладающей указанным свойством (скажем, гауссовской).

Более трудным является получение бесконечномерных свойств решений, которые могли бы рассматриваться как бесконечномерные аналоги абсолютной непрерывности и дифференцируемости. Есть результаты, согласно которым при некоторых специальных предположениях стационарное распределение бесконечномерной диффузии или решение эллиптического уравнения абсолютно непрерывно относительно заданной меры (в типичных случаях гауссовской), а ее плотность принадлежит некоторому классу Соболева; см. [3]-[5], [89], [144], [167]-[172] и ссылки в [26; гл. 12]. Следует заметить, что Н. В. Толмачев [173] построил пример бесконечномерной диффузии с гладкими коэффициентами и ограниченным и равномерно невырожденным коэффициентом диффузии, в котором переходные вероятности и стационарные распределения не имеют направлений непрерывности (в определенном выше смысле). Уже долго остается открытым вопрос, может ли такое случиться, если $A=I$. Некоторые 
положительные результаты для специальных сносов $b$ можно найти в цитированных выше работах, здесь мы упомянем лишь один типичный пример, который является результатом из [4], дающим решение проблемы, поставленной И. Шигекавой [174]. Для упрощения обозначений мы формулируем этот результат для пространства $\mathbb{R}^{\infty}$, хотя он справедлив для общих локально выпуклых пространств.

Теорема 3.2.2. Пусть $v=\left(v^{i}\right)$ - борелевское векторное поле на $X=\mathbb{R}^{\infty}$ со значениями в $H=l^{2}$ и творяющая уравнению (3.1.1) при $A=I u b(x)=-x+v(x)$, где $|v|_{H} \in L^{2}(\mu)$ $u l_{i} \in L^{2}(\mu), l_{i}(x)=x_{i}$. Тогда мера $\mu$ абсолютно непрерывна относительно гауссовской меры $\gamma$, являющейся счетной степенъю стандартной гауссовской меры на вещественной прямой.

Родственные проблемы для бесконечномерных многообразий изучаются в [24], [25], [175]-[178]. Относительно различных задач, связанных с существенной самосопряженностью и единственностью для бесконечномерных эллиптических операторов и порожденными ими полугруппами, см. [179]-[181], а также [26; гл. 12] и имеющиеся в этих работах ссылки. Все эти вопросы будут подробно рассмотрены в отдельном обзоре, посвященном только бесконечномерному случаю.

\section{Список литературы}

[1] A. Kolmogoroff, "Über die analytischen Methoden in der Wahrscheinlichkeitsrechnung", Math. Ann., 104:1 (1931), 415-458; рус. пер.: А. Н. Колмогоров, "Об аналитических методах в теории вероятностей", УМН, 5 (1938), 5-41.

[2] A. N. Kolmogoroff, "Zur Umkehrbarkeit der statistischen Naturgesetze", Math. Ann., 113:1 (1937), 766-772; рус. пер.: А. Н. Колмогоров, "Об обратимости статистических законов природы", Теория вероятностей и математическая статистика. Избранные труды, Наука, М., 1986, 197-204.

[3] V.I. Bogachev, M. Röckner, "Hypoellipticity and invariant measures for infinite dimensional diffusions", C. R. Acad. Sci. Paris Sér. I Math., 318:6 (1994), 553-558.

[4] V. I. Bogachev, M. Röckner, "Regularity of invariant measures on finite and infinite dimensional spaces and applications", J. Funct. Anal., 133:1 (1995), 168-223.

[5] V. I. Bogachev, N. Krylov, M. Röckner, "Regularity of invariant measures: the case of non-constant diffusion part", J. Funct. Anal., 138:1 (1996), 223-242.

[6] V. I. Bogachev, N. V. Krylov, M. Röckner, "Elliptic regularity and essential self-adjointness of Dirichlet operators on $\mathbb{R}^{n "}$, Ann. Scuola Norm. Sup. Pisa Cl. Sci. (4), 24:3 (1997), 451-461.

[7] S. Albeverio, V. Bogachev, M. Röckner, "On uniqueness of invariant measures for finite- and infinite-dimensional diffusions", Comm. Pure Appl. Math., 52:3 (1999), $325-362$.

[8] В.И. Богачев, М. Рёкнер, “Обобщение теоремы Хасьминского о существовании инвариантных мер для локально интегрируемых сносов", Теория вероятн. и ее примен., 45:3 (2000), 417-436; англ. пер.: V. Bogachev, M. Röckner, "A generalization of Khasminskii's theorem on the existence of invariant measures for locally integrable drifts", Theory Probab. Appl., 45:3 (2001), 363-378; В. И. Богачев, М. Рёкнер, "Письмо в редакцию: 'Обобщение теоремы Хасьминского о существовании инвариантных мер для локально интегрируемых сносов'”, Теория вероятн. и ее примен., $46: 3$ (2001), 600. 
[9] V. I. Bogachev, N. V. Krylov, M. Röckner, "On regularity of transition probabilities and invariant measures of singular diffusions under minimal conditions", Comm. Partial Differential Equations, 26:11-12 (2001), 2037-2080.

[10] V. I. Bogachev, M. Röckner, "Elliptic equations for measures on infinite dimensional spaces and applications", Probab. Theory Related Fields, 120:4 (2001), 445-496.

[11] V.I. Bogachev, M. Röckner, W. Stannat, "Uniqueness of invariant measures and essential $m$-dissipativity of diffusion operators on $L^{1 "}$, Infinite dimensional stochastic analysis (Amsterdam, 1999), Verh. Afd. Natuurkd. 1. Reeks. K. Ned. Akad. Wet., 52, R. Neth. Acad. Arts Sci., Amsterdam, 2000, 39-54.

[12] В. И. Богачев, М. Рёкнер, В. Штаннат, "Единственность решений эллиптических уравнений и единственность инвариантных мер диффузий", Матем. сб., 193:7 (2002), 3-36; англ. пер.: V. I. Bogachev, M. Rockner, W. Stannat, "Uniqueness of solutions of elliptic equations and uniqueness of invariant measures of diffusions", Sb. Math., 193:7-8 (2002), 945-976.

[13] А.И. Кириллов, "О двух математических проблемах канонического квантования. I", ТМФ, 87:1 (1991), 22-33; англ. пер.: A. I. Kirillov, "Two mathematical problems of canonical quantization. I", Theoret. Math. Phys., 87:1 (1991), 345-353; "II", 87:2 (1991), 163-172; англ. пер.: "II", 87:2 (1991), 447-454; "III: Стохастическая механика вакуума", 91:3 (1992), 377-395; англ. пер.: "III: Stochastic vacuum mechanics", 91:3 (1992), 591-603; "IV", 93:2 (1992), 249-263; англ. пер.: "IV", 93:2 (1992), 1251-1261.

[14] А.И. Кириллов, "Бесконечномерный анализ и квантовая теория как исчисления семимартингалов", УМH, 49:3 (1994), 43-92; англ. пер.: A. I. Kirillov, "Infinite-dimensional analysis and quantum theory as semimartingale calculus", Russian Math. Surveys, 49:3 (1994), 43-95.

[15] А.И. Кириллов, "Поле типа sin-Gordon в пространстве-времени произвольной размерности: существование меры Нельсона", ТМФ, 98:1 (1994), 12-28; англ. пер.: A. I. Kirillov, "Field of sine-Gordon type in spacetime of arbitrary dimension: Existence of the Nelson measure", Theoret. Math. Phys., 98:1 (1994), 8-19; "Поле типа sin-Gordon в пространстве-времени произвольной размерности. II: Стохастическое квантование", ТМФ, 105:2 (1995), 179-197; англ. пер.: "Sine-Gordon type field in spacetime of arbitrary dimension. II: Stochastic quantization", 105:2 (1995), 1329-1345.

[16] А. И. Кириллов, "О восстановлении мер по их логарифмическим производным", Изв. РАН. Сер. матем., 59:1 (1995), 121-138; англ. пер.: А. I. Kirillov, "On the reconstruction of measures from their logarithmic derivatives", Izv. Math., 59:1 (1995), 121-139.

[17] Z.M. Ma, M. Röckner, Introduction to the theory of (non-symmetric) Dirichlet forms, Universitext, Springer-Verlag, Berlin, 1992.

[18] V. I. Bogachev, G. Da Prato, M. Röckner, "Existence of solutions to weak parabolic equations for measures", Proc. London Math. Soc. (3), 88:3 (2004), 753-774.

[19] V. I. Bogachev, G. Da Prato, M. Röckner, "On parabolic equations for measures", Comm. Partial Differential Equations, 33:1-3 (2008), 397-418.

[20] V.I. Bogachev, G. Da Prato, M. Röckner, W. Stannat, "Uniqueness of solutions to weak parabolic equations for measures", Bull. Lond. Math. Soc., 39:4 (2007), 631-640.

[21] В. И. Богачев, М. Рёкнер, С. В. Шапошников, "Глобальная регулярность и оценки решений параболических уравнений", Теория вероятн. и ее примен., 50:4 (2005), 652-674; англ. пер.: V.I. Bogachev, M. Rockner, S. V. Shaposhnikov, "Global regularity and bounds for solutions of parabolic equations for probability measures", Theory Probab. Appl., 50:4 (2006), 561-581. 
[22] В.И.Богачев, М. Рёкнер, С.В.Шапошников, "Оценки плотностей стационарных распределений и переходных вероятностей диффузионных процессов", Теория вероятн. и ее примен., 52:2 (2007), 240-270; англ. пер.: V.I. Bogachev, M. Röckner, S. V. Shaposhnikov, "Estimates of densities of stationary distributions and transition probabilities of diffusion processes", Theory Probab. Appl., 52:2 (2008), 209-236.

[23] В. И. Богачев, М. Рёкнер, С. В. Шапошников, "Положительные плотности переходных вероятностей диффузионных процессов", Теория вероятн. и ее примен., 53:2 (2008), 213-239; англ. пер.: V. I. Bogachev, M. Röckner, S. V. Shaposhnikov, "Positive densities of transition probabilities of diffusion processes", Theory Probab. Appl., 53:2 (2009), 194-215.

[24] V. I. Bogachev, M. Röckner, F.-Y. Wang, "Elliptic equations for invariant measures on finite and infinite dimensional manifolds", J. Math. Pures Appl. (9), 80:2 (2001), $177-221$.

[25] V.I. Bogachev, M. Röckner, F.-Y. Wang, "Invariance implies Gibbsian: some new results", Comm. Math. Phys., 248:2 (2004), 335-355.

[26] В. И. Богачев, Дифференцируемые меры и исчисление Маллявэна, НИЦ "Регулярная и хаотическая динамика", М.-Ижевск, 2008.

[27] P. Sjögren, "On the adjoint of an elliptic linear differential operator and its potential theory", Ark. Mat., 11:1-2 (1973), 153-165.

[28] A. Bensoussan, Perturbation methods in optimal control, Wiley/Gauthier-Villars Series in Modern Applied Mathematics, Wiley, Chichester; Gauthier-Villars, Montrouge, 1988.

[29] C. B. Morrey, Multiple integrals in the calculus of variations, Grundlehren Math. Wiss., 130, Springer-Verlag, New York, 1966.

[30] С. В. Шапошников, “Оценка Морри соболевских норм решений эллиптических уравнений", Матем. заметки, 79:3 (2006), 450-469; англ. пер.: S. V. Shaposhnikov, "On Morrey's estimate of the Sobolev norms of solutions of elliptic equations", Math. Notes, 79:3-4 (2006), 413-430.

[31] С. В. Шапошников, "О внутренних оценках соболевских норм решений эллиптических уравнений”, Матем. заметки, 83:2 (2008), 316-320; англ. пер.: S. V. Shaposhnikov, "On interior estimates of the Sobolev norms of solutions of elliptic equations", Math. Notes, 83:1-2 (2008), 285-289.

[32] О.В. Бесов, В.П. Ильин, С.М.Никольский, Интегральные представления функиий и теоремъ вложения, Наука, М., 1975; англ. пер.: О. V. Besov, V.P. Il'in, S. M. Nikol'skii, Integral representations of functions and imbedding theorems. Vol. I, Scripta Series in Mathematics, Winston, Washington, DC; Halsted Press, New York-Toronto-London, 1978; Vol. II, Scripta Series in Mathematics, Winston, Washington, DC; Halsted Press, New York-Toronto-London, 1979.

[33] С. В. Шапошников, "Положительность инвариантных мер диффузионных процессов", Докл. РАН, 415:2 (2007), 174-179; англ. пер.: S. V. Shaposhnikov, "Positiveness of invariant measures of diffusion processes", Dokl. Math., 76:1 (2007), 533-538.

[34] W. Littman, "A strong maximum principle for weakly $L$-subharmonic functions", J. Math. Mech., 8 (1959), 761-770.

[35] P. Bauman, "Positive solutions of elliptic equations in nondivergent form and their adjoints", Ark. Mat., 22:1-2 (1984), 153-173.

[36] L. Escauriaza, "Weak type- $(1,1)$ inequalities and regularity properties of adjoint and normalized adjoint solutions to linear nondivergence form operators with VMO coefficients", Duke Math. J., 74:1 (1994), 177-201.

[37] N. V. Krylov, "Parabolic and elliptic equations with VMO coefficients", Comm. Partial Differential Equations, 32:1-3 (2007), 453-475. 
[38] N. V. Krylov, Lectures on elliptic and parabolic equations in Sobolev spaces, Grad. Stud. Math., 96, Amer. Math. Soc., Providence, RI, 2008.

[39] N. V. Krylov, "Second-order elliptic equations with variably partially VMO coefficients", J. Funct. Anal., 257:6 (2009), 1695-1712; arXiv: 0807.0926, 2008.

[40] T. Jin, V. Maz'ya, J. Van Schaftingen, "Pathological solutions to elliptic problems in divergence form with continuous coefficients", C. R. Math. Acad. Sci. Paris, 347:13-14 (2009), 773-778; arXiv: 0904.1674v1, 2009.

[41] А. Ю. Веретенников, Э. Парду, "О гладкости инвариантной меры цепи Маркова по параметру", Докл. РАН, 370:2 (2000), 158-160; англ. пер.: A. Yu. Veretennikov, É. Pardoux, "On the smoothness of an invariant measure of a Markov chain with respect to a parameter", Dokl. Math., 61:1 (2000), 31-33.

[42] Р. З. Хасьминский, "Эргодические свойства возвратных диффузионных процессов и стабилизация решений задачи Коши для параболических уравнений", Теория вероятн. и ее примен., 5:2 (1960), 196-214; англ. пер.: R. Z. Khas'minskii, "Ergodic properties of recurrent diffusion processes and stabilization of the solution to the Cauchy problem for parabolic equations", Theory Probab. Appl., 5:2 (1960), 179-196.

[43] Р. З. Хасьминский, Устойчивость систем дифференциалъных уравнений при случайных возмущениях их параметров, Наука, M., 1969; англ. пер.: R. Z. Has'minskii, Stochastic stability of differential equations, Monogr. Textbooks Mech. Solids Fluids: Mech. Anal., 7, Sijthoff and Noordhoff, Germantown, MD, 1980.

[44] N. S. Trudinger, "Linear elliptic operators with measurable coefficients", Ann. Scuola Norm. Sup. Pisa (3), 27 (1973), 265-308.

[45] N.S. Trudinger, "Maximum principles for linear, non-uniformly elliptic operators with measurable coefficients", Math. Z., 156:3 (1977), 291-301.

[46] Д. Гилбарг, Н. Трудингер, Эллиптические дифференииальные уравнения с частными производными второго порядка, Наука, М., 1989; пер. с англ.: D. Gilbarg, N. S. Trudinger, Elliptic partial differential equations of second order, Grundlehren Math. Wiss., 224, Springer-Verlag, Berlin-New York, 1977.

[47] A. Yu. Veretennikov, "On polynomial mixing bounds for stochastic differential equations", Stochastic Process. Appl., 70:1 (1997), 115-127.

[48] V.I. Bogachev, M. Röckner, T.S. Zhang, "Existence and uniqueness of invariant measures: an approach via sectorial forms", Appl. Math. Optim., 41:1 (2000), 87-109.

[49] V.A. Liskevich, Yu. A. Semenov, "Dirichlet operators: a priori estimates and the uniqueness problem", J. Funct. Anal., 109:1 (1992), 199-213.

[50] W. Stannat, "(Nonsymmetric) Dirichlet operators on $L^{1}$ : existence, uniqueness and associated Markov processes", Ann. Scuola Norm. Sup. Pisa Cl. Sci. (4), 28:1 (1999), 99-140.

[51] M. Röckner, T. S. Zhang, "Uniqueness of generalized Schrödinger operators. Part II", J. Funct. Anal., 119:2 (1994), 455-467.

[52] E. Schrödinger, "Über die Umkehrung der Naturgesetze", Sitzungsber. Preuss. Akad. Wiss. Phys.-Math. Kl., 1931, № 8-9, 144-153.

[53] U. G. Haussmann, É. Pardoux, "Time reversal of diffusions", Ann. Probab., 14:4 (1986), 1188-1205.

[54] H. Föllmer, "Random fields and diffusion processes", École d'Été de Probabilités de Saint-Flour XV-XVII, Lect. Notes in Math., 1362, Springer, Berlin, 1988, 101-203.

[55] A. Millet, D. Nualart, M. Sanz, "Integration by parts and time reversal for diffusion processes", Ann. Probab., 17:1 (1989), 208-238.

[56] A. Millet, D. Nualart, M. Sanz, "Time reversal for infinite-dimensional diffusions", Probab. Theory Relat. Fields, 82:3 (1989), 315-347.

[57] P. Echeverría, "A criterion for invariant measures of Markov processes", Z. Wahrscheinlichkeitstheor. verw. Geb., 61:1 (1982), 1-16. 
[58] A. Albanese, L. Lorenzi, E. Mangino, " $L^{p}$-uniqueness for elliptic operators with unbounded coefficients in $\mathbb{R}^{N ",}$ J. Funct. Anal., 256:4 (2009), 1238-1257.

[59] A. A. Albanese, E. M. Mangino, "Cores for Feller semigroups with an invariant measure", J. Differential Equations, 225:1 (2006), 361-377; A. A. Albanese, E. M. Mangino, "Corrigendum to "Cores for Feller semigroups with an invariant measure'", J. Differential Equations, 244:11 (2008), 2980-2982.

[60] R. N. Bhattacharya, "Criteria for recurrence and existence of invariant measures for multidimensional diffusions", Ann. Probab., 6:4 (1978), 541-553; R. N. Bhattacharya, "Correction Note: Correction to 'Criteria for recurrence and existence of invariant measures for multidimensional diffusions'”, Ann. Probab., 8:6 (1980), 1194-1195.

[61] R. N. Bhattacharya, S. Ramasubramanian, "Recurrence and ergodicity of diffusions", J. Multivariate Anal., 12:1 (1982), 95-122.

[62] Y. Miyahara, "Invariant measures of ultimately bounded stochastic processes", Nagoya Math. J., 49 (1973), 149-153.

[63] M. Zakai, "A Lyapunov criterion for the existence of stationary probability distributions for systems perturbed by noise", SIAM J. Control, 7:3 (1969), 390-397.

[64] L. Arnold, W. Kliemann, "Qualitative theory of stochastic systems", Probabilistic analysis and related topics, Vol. 3, Academic Press, New York, 1983, 1-79.

[65] А. А. Боровков, Эргодичность и устойчивость случайных процессов, Эдиториал УРСС, М., 1994; англ. пер.: А. А. Borovkov, Ergodicity and stability of stochastic processes, Wiley, Chichester, 1998.

[66] M. Cohen de Lara, "Geometric and symmetry properties of a nondegenerate diffusion process", Ann. Probab., 23:4 (1995), 1557-1604.

[67] А. Ю. Веретенников, С. А. Клоков, "О субэкспоненциальной скорости перемешивания для марковских процессов", Теория вероятн. и ее примен., 49:1 (2004), 21-35; англ. пер.: S. A. Klokov, A. Yu. Veretennikov, "On subexponential mixing rate for Markov processes", Theory Probab. Appl., 49:1 (2005), 110-122.

[68] H. J. Kushner, Stochastic stability and control, Math. Sci. Eng., 33, Academic Press, New York, 1967; рус. пер.: Г. Дж. Кушнер, Стохастическая устойчивость и управление, Мир, М., 1969.

[69] S. Meyn, R. L. Tweedie, Markov chains and stochastic stability, Prologue by Peter W. Glynn, 2nd ed., Cambridge Univ. Press, Cambridge, 2009.

[70] É. Pardoux, A. Yu. Veretennikov, "On the Poisson equation and diffusion approximation. I", Ann. Probab., 29:3 (2001), 1061-1085; "II", 31:3 (2003), 1166-1192; "III", 33:3 (2005), 1111-1133.

[71] R. G. Pinsky, Positive harmonic functions and diffusion, Cambridge Stud. Adv. Math., 45, Cambridge Univ. Press, Cambridge, 1995.

[72] В. М. Шуренков, Эргодические прочессы Маркова, Наука, М., 1989.

[73] А. В. Скороход, Асимптотические методы теории стохастических дифференциальных уравнений, Наукова Думка, Киев, 1987; англ. пер.: A. V. Skorokhod, Asymptotic methods in the theory of stochastic differential equations, Transl. Math. Monogr., Amer. Math. Soc., Providence, RI, 1989.

[74] А. Ю. Веретенников, "О полиномиальном перемешивании и скорости сходимости для стохастических дифференциальных и разностных уравнений", Теория вероятн. и ее примен., 44:2 (1999), 312-327; англ. пер.: А. Yu. Veretennikov, "On polynomial mixing and convergence rate for stochastic difference and differential equations", Theory Probab. Appl., 44:2 (1999), 361-374.

[75] G. Da Prato, A. Lunardi, "On a class of self-adjoint elliptic operators in $L^{2}$ spaces with respect to invariant measures", J. Differential Equations, 234:1 (2007), 54-79.

[76] B. Farkas, A. Lunardi, "Maximal regularity for Kolmogorov operators in $L^{2}$ spaces with respect to invariant measures", J. Math. Pures Appl. (9), 86:4 (2006), 310-321. 
[77] L. Lorenzi, M. Bertoldi, Analytical methods for Markov semigroups, Pure Appl. Math. (Boca Raton), 283, Chapman \& Hall/CRC, Boca Raton, FL, 2007.

[78] G. Metafune, D. Pallara, M. Wacker, "Compactness properties of Feller semigroups", Studia Math., 153:2 (2002), 179-206.

[79] D. Bakry, "L'hypercontractivité et son utilisation en théorie des semigroupes", Lectures on probability theory (Saint-Flour, 1992), Lecture Notes in Math., 1581, Springer, Berlin, 1994, 1-114.

[80] P. Cattiaux, A. Guillin, F.-Y. Wang, L. Wu, "Lyapunov conditions for super Poincaré inequalities", J. Funct. Anal., 256:6 (2009), 1821-1841.

[81] Y. Fujita, "On a critical role of Ornstein-Uhlenbeck operators in the Poincaré inequality", Differential Integral Equations, 19:12 (2006), 1321-1332.

[82] M. Ledoux, "On an integral criterion for hypercontractivity of diffusion semigroups and extremal functions", J. Funct. Anal., 105:2 (1992), 444-465.

[83] M. Röckner, F.-Y. Wang, "Supercontractivity and ultracontractivity for (non-symmetric) diffusion semigroups on manifolds", Forum Math., 15:6 (2003), 893-921.

[84] С. В. Шапошников, "О неединственности решений эллиптических уравнений для вероятностных мер”, Докл. РАН, 420:3 (2008), 320-323; англ. пер.: S. V. Shaposhnikov, "The nonuniqueness of solutions to elliptic equations for probability measures", Dokl. Math., 77:3 (2008), 401-403.

[85] S. V. Shaposhnikov, "On nonuniqueness of solutions to elliptic equations for probability measures", J. Funct. Anal., 254:10 (2008), 2690-2705.

[86] В. В. Жиков, "Замечания о единственности решения задачи Дирихле для эллиптического уравнения второго порядка с младшими членами", Функи. анализ и его прил., 38:3 (2004), 15-28; англ. пер.: V. V. Zhikov, "Remarks on the uniqueness of a solution of the Dirichlet problem for second-order elliptic equations with lower-order terms", Funct. Anal. Appl., 38:3 (2004), 173-183.

[87] S. R. S. Varadhan, Lectures on diffusion problems and partial differential equations, Tata Inst. Fund. Res. Lectures on Math. and Phys., 64, Tata Institute of Fundamental Research, Bombay, 1980.

[88] J.L. Doob, "Asymptotic properties of Markoff transitions probabilities", Trans. Amer. Math. Soc., 63:3 (1948), 393-421.

[89] G. Da Prato, J. Zabczyk, Ergodicity for infinite-dimensional systems, London Math. Soc. Lecture Note Ser., 229, Cambridge Univ. Press, Cambridge, 1996.

[90] P. Cattiaux, M. Fradon, "Entropy, reversible diffusion processes, and Markov uniqueness", J. Funct. Anal., 138:1 (1996), 243-272.

[91] A. Eberle, Uniqueness and non-uniqueness of semigroups generated by singular diffusion operators, Lecture Notes in Math., 1718, Springer, Berlin, 1999.

[92] A. Eberle, " $L^{p}$ uniqueness of non-symmetric diffusion operators with singular drift coefficients. I: The finite-dimensional case", J. Funct. Anal., 173:2 (2000), 328-342.

[93] M. Kolb, "On the strong uniqueness of some finite dimensional Dirichlet operators", Infin. Dimens. Anal. Quantum Probab. Relat. Top., 11:2 (2008), 279-293.

[94] V. Liskevich, "On the uniqueness problem for Dirichlet operators", J. Funct. Anal., 162:1 (1999), 1-13.

[95] В. И. Богачев, М. Рёкнер, "Об $L^{p}$-единственности симметричных диффузионных операторов на римановых многообразиях", Матем. сб., 194:7 (2003), 15-24; англ. пер.: V. I. Bogachev, M. Röckner, "On $L^{p}$-uniqueness of symmetric diffusion operators on Riemannian manifolds", Sb. Math., 194:7 (2003), 969-978.

[96] L. M. Wu, Y. Zhang, "A new topological approach to the $L^{\infty}$-uniqueness of operators and the $L^{1}$-uniqueness of Fokker-Planck equations", J. Funct. Anal., 241:2 (2006), 557-610.

[97] G. Metafune, D. Pallara, A. Rhandi, "Global regularity of invariant measures", J. Funct. Anal., 223:2 (2005), 396-424. 
[98] X.-D. Li, "Liouville theorems for symmetric diffusion operators on complete Riemannian manifolds", J. Math. Pures Appl. (9), 84:10 (2005), 1295-1361.

[99] V. I. Bogachev, N. V. Krylov, M. Röckner, "Elliptic equations for measures: regularity and global bounds of densities", J. Math. Pures Appl. (9), 85:6 (2006), 743-757.

[100] A.-B. Cruzeiro, P. Malliavin, "Nonperturbative construction of invariant measure through confinement by curvature", J. Math. Pures Appl. (9), 77:6 (1998), 527-537.

[101] В. И. Богачев, М. Рёкнер, С. В. Шаошников, "Нижние оценки плотностей решений эллиптических уравнений для мер”, Докл. РАН, 426:2 (2009), 156-161; англ. пер.: V. I. Bogachev, M. Röckner, S. V. Shaposhnikov, Dokl. Math., 79:3 (2009), 329-334.

[102] E. Nualart, "Exponential divergence estimates and heat kernel tail", C. R. Math. Acad. Sci. Paris, 338:1 (2004), 77-80.

[103] P. Malliavin, E. Nualart, "Density minoration of a strongly non-degenerated random variable", J. Funct. Anal., 256:12 (2009), 4197-4214.

[104] V. I. Bogachev, E. Mayer-Wolf, "Absolutely continuous flows generated by Sobolev class vector fields in finite and infinite dimensions", J. Funct. Anal., 167:1 (1999), $1-68$.

[105] M. Scheutzow, H. von Weizsäcker, "Which moments of a logarithmic derivative imply quasiinvariance?", Doc. Math., 3 (1998), 261-272.

[106] D. W. Stroock, S. R. S. Varadhan, Multidimensional diffusion processes, Grundlehren Math. Wiss., 233, Springer-Verlag, Berlin-New York, 1979.

[107] W. Stannat, "Time-dependent diffusion operators on $L^{1 "}$, J. Evol. Equ., 4:4 (2004), 463-495.

[108] Н. В. Крылов, Управляемые диффузионные прочессы, Теория вероятностей и математическая статистика, Наука, М., 1977; англ. пер.: N. V. Krylov, Controlled diffusion processes, Appl. Math., 14, Springer-Verlag, New York-Berlin, 1980.

[109] E. B. Fabes, C.E. Kenig, "Examples of singular parabolic measures and singular transition probability densities", Duke Math. J., 48:4 (1981), 845-856.

[110] M. V. Safonov, "An example of a diffusion process with the singular distribution at a fixed moment", Abstracts of Communications of the Third International Vilnius Conference on Probability Theory and Mathematical Statistics, Vol. II, Vilnius, 1981, 133-134.

[111] Н.И. Портенко, Обобщенные диффузионные процессы, Наукова Думка, Киев, 1982; англ. пер.: N. I. Portenko, Generalized diffusion processes, Transl. Math. Monogr., 83, Amer. Math. Soc., Providence, RI, 1990.

[112] P. Cattiaux, C. Léonard, "Minimization of the Kullback information of diffusion processes", Ann. Inst. H. Poincaré Probab. Statist., 30:1 (1994), 83-132; P. Cattiaux, C. Léonard, "Correction to: 'Minimization of the Kullback information of diffusion processes'”, Ann. Inst. H. Poincaré Probab. Statist., 31:4 (1995), 705-707.

[113] G. Metafune, D. Pallara, A. Rhandi, "Global properties of transition probabilities of singular diffusions", Теория вероятн. и ее примен., 54:1 (2009), 116-148; Theory Probab. Appl., 54:1 (2009).

[114] T. J. Lyons, W. A. Zheng, "Diffusion processes with nonsmooth diffusion coefficients and their density functions", Proc. Roy. Soc. Edinburgh Sect. A, 115:3-4 (1990), 231-242.

[115] О.А. Ладыженская, В.А. Солонников, Н.Н. Уральцева, Линейные и квазилинейные уравнения параболического типа, Наука, М., 1967; англ. пер.: O.A. Ladyženskaja, V.A. Solonnikov, N. M. Ural'ceva, Linear and quasilinear equations of parabolic type, Transl. Math. Monogr., 23, Amer. Math. Soc., Providence, RI, 1968.

[116] G.M. Lieberman, Second order parabolic differential equations, World Scientific Publ., River Edge, NJ, 1996. 
[117] A. Lunardi, Analytic semigroups and optimal regularity in parabolic problems, Progr. Nonlinear Differential Equations Appl., 16, Birkhäuser, Basel, 1995.

[118] S. D. Eidelman, S. D. Ivasyshen, A. N. Kochubei, Analytic methods in the theory of differential and pseudo-differential equations of parabolic type, Oper. Theory Adv. Appl., 152, Birkhäuser, Basel, 2004.

[119] Ф. О. Порпер, С. Д. Эйдельман, “Двусторонние оценки фундаментальных решений параболических уравнений второго порядка и некоторые их приложения", УМH, 39:3 (1984), 107-156; англ. пер.: F. O. Porper, S. D. Eidel'man, "Two-sided estimates of fundamental solutions of second-order parabolic equations, and some applications", Russian Math. Surveys, 39:3 (1984), 119-178.

[120] Ф. О. Порпер, С. Д. Эйдельман, "Свойства решений параболических уравнений второго порядка с членами низшего порядка", Тр. МMO, 54 (1992), 118-159; англ. пер.: F. O. Porper, S. D. Eidel'man, "Properties of solutions of second-order parabolic equations with lower-order terms", Trans. Moscow Math. Soc., 1993, 101-137.

[121] S. J. Sheu, "Some estimates of the transition density of a nondegenerate diffusion Markov process", Ann. Probab., 19:2 (1991), 538-561.

[122] L. Escauriaza, "Bounds for the fundamental solution of elliptic and parabolic equations in nondivergence form", Comm. Partial Differential Equations, 25:5-6 (2000), 821-845.

[123] W. Arendt, G. Metafune, D. Pallara, "Gaussian estimates for elliptic operators with unbounded drift", J. Math. Anal. Appl., 338:1 (2008), 505-517.

[124] S. Fornaro, N. Fusco, G. Metafune, D. Pallara, "Sharp upper bounds for the density of some invariant measures", Proc. Roy. Soc. Edinburgh Sect. A, 139:6 (2009), $1145-1161$.

[125] C. Spina, "Kernel estimates for a class of Kolmogorov semigroups", Arch. Math. (Basel), 91:3 (2008), 265-279.

[126] В.Н. Денисов, "О поведении решений параболических уравнений при больших значениях времени", УМH, 60:4 (2005), 145-212; англ. пер.: V. N. Denisov, "On the behaviour of solutions of parabolic equations for large values of time", Russian Math. Surveys, 60:4 (2005), 721-790.

[127] D. G. Aronson, J. Serrin, "Local behavior of solutions of quasilinear parabolic equations", Arch. Ration. Mech. Anal., 25:2 (1967), 81-122.

[128] J. Moser, "A Harnack inequality for parabolic differential equations", Comm. Pure Appl. Math., 17:1 (1964), 101-134; J. Moser, "Correction to: 'A Harnack inequality for parabolic differential equations'", Comm. Pure Appl. Math., 20:1 (1967), 231-236.

[129] С. В. Шапошников, "Нижние оценки плотностей решений параболических уравнений для мер", Докл. РАН, 429:5 (2009), 600-604.

[130] V.I. Bogachev, G. Da Prato, M. Röckner, "Fokker-Planck equations and maximal dissipativity for Kolmogorov operators with time dependent singular drifts in Hilbert spaces", J. Funct. Anal., 256:4 (2009), 1269-1298.

[131] В. И. Богачев, Дж. Да Прато, М. Рёкнер, "Бесконечномерные операторы Колмогорова с зависящими от времени коэффициентами сноса", Докл. РАH, 419:5 (2008), 587-591; англ. пер.: V. I. Bogachev, G. Da Prato, M. Röckner, "Infinite dimensional Kolmogorov operators with time dependent drift coefficients", Dokl. Math., 77:2 (2008), 276-280.

[132] G. Da Prato, Kolmogorov equations for stochastic PDEs, Adv. Courses Math. CRM Barcelona, Birkhäuser, Basel, 2004.

[133] В.И. Богачев, М. Рёкнер, С. В. Шапошников, "Нелинейные эволюционные и транспортные уравнения для мер", Докл. РАН, 429:1 (2009), 7-11; англ. пер.: Dokl. Math., 80:3 (2009), 785-789. 
[134] В. И. Богачев, Гауссовские меры, Наука, М., 1997; англ. пер.: V. I. Bogachev, Gaussian measures, Math. Surveys Monogr., 62, Amer. Math. Soc., Providence, RI, 1998.

[135] S. Assing, R. Manthey, "Invariant measures for stochastic heat equations with unbounded coefficients", Stochastic Process. Appl., 103:2 (2003), 237-256.

[136] Z. Brzeźniak, Y. Li, "Asymptotic compactness and absorbing sets for 2D stochastic Navier-Stokes equations on some unbounded domains", Trans. Amer. Math. Soc., 358:12 (2006), 5587-5629.

[137] S. Cerrai, M. Freidlin, "Averaging principle for a class of stochastic reaction-diffusion equations", Probab. Theory Related Fields, 144:1-2 (2009), 137-177.

[138] A. Chojnowska-Michalik, "Transition semigroups for stochastic semilinear equations on Hilbert spaces", Dissertationes Math. (Rozprawy Mat.), 2001, № 396.

[139] G. Da Prato, A. Debussche, "Ergodicity for the 3D stochastic Navier-Stokes equations", J. Math. Pures Appl. (9), 82:8 (2003), 877-947.

[140] W. E, J. C. Mattingly, Ya. Sinai, "Gibbsian dynamics and ergodicity for the stochastically forced Navier-Stokes equation", Comm. Math. Phys., 224:1 (2001), 83-106.

[141] J.-P. Eckmann, M. Hairer, "Invariant measures for stochastic partial differential equations in unbounded domains", Nonlinearity, 14:1 (2001), 133-151.

[142] A. Es-Sarhir, W. Stannat, "Invariant measures for semilinear SPDE's with local Lipschitz drift coefficients and applications", J. Evol. Equ., 8:1 (2008), 129-154.

[143] F. Flandoli, Regularity theory and stochastic flows for parabolic SPDEs, Stochastics Monographs, 9, Gordon and Breach, Yverdon, 1995.

[144] B. Goldys, B. Maslowski, "Lower estimates of transition densities and bounds on exponential ergodicity for stochastic PDE's", Ann. Probab., 34:4 (2006), 1451-1496.

[145] M. Hairer, J. C. Mattingly, "Spectral gaps in Wasserstein distances and the 2D stochastic Navier-Stokes equations", Ann. Probab., 36:6 (2008), 2050-2091.

[146] B. Maslowski, J. Seidler, "Invariant measures for nonlinear SPDE's: uniqueness and stability", Arch. Math. (Brno), 34:1 (1998), 153-172.

[147] M. Röckner, F.-Y. Wang, "Concentration of invariant measures for stochastic generalized porous media equations", Infin. Dimens. Anal. Quantum Probab. Relat. Top., 10:3 (2007), 397-409.

[148] A. Shirikyan, "Qualitative properties of stationary measures for three-dimensional Navier-Stokes equations", J. Funct. Anal., 249:2 (2007), 284-306.

[149] С. Альбеверио, Ю. Кондратьев, Т. Пазурек, М. Рекнер, “Существование и априорные оценки для евклидовых гиббсовских состояний”, Тр. MMO, 67 (2006), 3-103; англ. пер.: S. Albeverio, Yu. Kondrat'ev, T. Pazurek, M. Röckner, "Existence and a priori estimates for Euclidean Gibbs states", Trans. Moscow Math. Soc., 2006, $1-85$.

[150] S. Albeverio, Yu. G. Kondratiev, M. Röckner, T. V. Tsikalenko, "A priori estimates for symmetrizing measures and their applications to Gibbs states", J. Funct. Anal., 171:2 (2000), 366-400.

[151] P. Cattiaux, S. Roelly, H. Zessin, "Une approche gibbsienne des diffusions browniennes infini-dimensionnelles", Probab. Theory Related Fields, 104:2 (1996), 147-179.

[152] J. Fritz, "Stationary measures of stochastic gradient systems, infinite lattice models", Z. Wahrscheinlichkeitstheor. verw. Geb., 59:4 (1982), 479-490.

[153] J. Fritz, T. Funaki, J. L. Lebowitz, "Stationary states of random Hamiltonian systems", Probab. Theory Related Fields, 99:2 (1994), 211-236.

[154] R. A. Holley, D. W. Stroock, "In one and two dimensions, every stationary measure for a stochastic Ising model is a Gibbs state", Comm. Math. Phys., 55:1 (1977), $37-45$.

[155] M. Namiki, Stochastic quantization, Lecture Notes in Phys. New Ser. m Monogr., 9, Springer-Verlag, Berlin, 1992. 
[156] P.-L. Chow, R.Z. Khasminskii, "Stationary solutions of nonlinear stochastic evolution equations", Stochastic Anal. Appl., 15:5 (1997), 671-699.

[157] G. Leha, G. Ritter, "Lyapunov-type conditions for stationary distributions of diffusion processes in Hilbert spaces", Stochastics, 48:3-4 (1994), 195-225.

[158] G. Leha, G. Ritter, A. Wakolbinger, "An improved Lyapunov-function approach to the behavior of diffusion processes in Hilbert spaces", Stochastic Anal. Appl., 15:1 (1997), 59-89.

[159] V.I. Bogachev, "Differentiable measures and the Malliavin calculus", J. Math. Sci. (New York), 87:4 (1997), 3577-3731.

[160] S. Albeverio, M. Röckner, "Stochastic differential equations in infinite dimensions: solutions via Dirichlet forms", Probab. Theory Related Fields, 89:3 (1991), 347-386.

[161] H. Long, "Necessary and sufficient conditions for the symmetrizability of differential operators over infinite dimensional state spaces", Forum Math., 12:2 (2000), 167-196.

[162] В. И. Богачев, Дж. Да Прато, М. Рёкнер, "Параболические уравнения для мер на бесконечномерных пространствах", Докл. РАН, 421:4 (2008), 439-444; англ. пер.: V.I. Bogachev, G. Da Prato, M. Röckner, "Parabolic equations for measures on infinite-dimensional spaces", Dokl. Math., 78:1 (2008), 544-549.

[163] L. Manca, "Kolmogorov equations for measures", J. Evol. Equ., 8:2 (2008), 231-262; arXiv: math/0703654, 2007.

[164] В.И. Богачев, Дж. Да Прато, М. Рёкнер, "Инвариантные меры обобщенных стохастических уравнений пористых сред”, Докл. РАН, 396:1 (2004), 7-11; англ. пер.: V. I. Bogachev, G. Da Prato, M. Röckner, "Invariant measures of generalized stochastic porous medium equations", Dokl. Math., 69:3 (2004), 321-325.

[165] V. Barbu, V. I. Bogachev, G. Da Prato, M. Röckner, "Weak solutions to the stochastic porous media equation via Kolmogorov equations: The degenerate case", J. Funct. Anal., 237:1 (2006), 54-75.

[166] A. Agrachev, S. Kuksin, A. Sarychev, A. Shirikyan, "On finite-dimensional projections of distributions for solutions of randomly forced 2D Navier-Stokes equations", Ann. Inst. H. Poincaré Probab. Statist., 43:4 (2007), 399-415.

[167] V. I. Bogachev, G. Da Prato, M. Röckner, "Regularity of invariant measures for a class of perturbed Ornstein-Uhlenbeck operators", NoDEA Nonlinear Differential Equations Appl., 3:2 (1996), 261-268.

[168] G. Da Prato, A. Debussche, "Absolute continuity of the invariant measures for some stochastic PDEs", J. Statist. Phys., 115:1-2 (2004), 451-468.

[169] G. Da Prato, A. Debussche, B. Goldys, "Some properties of invariant measures of non symmetric dissipative stochastic systems", Probab. Theory Related Fields, 123:3 (2002), 355-380.

[170] A. Es-Sarhir, "Sobolev regularity of invariant measures for generalized Ornstein-Uhlenbeck operators", Infin. Dimens. Anal. Quantum Probab. Relat. Top., 9:4 (2006), 595-606.

[171] B. Gaveau, J.-M. Moulinier, "Régularité des mesures et perturbations stochastiques de champs de vecteurs sur des espaces de dimension infinie", Publ. Res. Inst. Math. Sci., 21:3 (1985), 593-616.

[172] M. Hino, "Existence of invariant measures for diffusion processes on a Wiener space", Osaka J. Math., 35:3 (1998), 717-734.

[173] Н. А. Толмачев, "О гладкости и сингулярности инвариантных мер и переходных вероятностей бесконечномерных диффузий”, Теория вероятн. и ее примен., 43:4 (1998), 798-808; англ. пер.: N. A. Tolmachev, "On the smoothness and singularity of invariant measures and transition probabilities of infinite-dimensional diffusions", Theory Probab. Appl., 43:4 (1999), 655-664. 
[174] I. Shigekawa, "Existence of invariant measures of diffusions on an abstract Wiener space", Osaka J. Math., 24:1 (1987), 37-59.

[175] A.F. Ramírez, "Relative entropy and mixing properties of infinite dimensional diffusions", Probab. Theory Related Fields, 110:3 (1998), 369-395.

[176] A.F. Ramírez, "Uniqueness of invariant product measures for elliptic infinite dimensional diffusions and particle spin systems", ESAIM Probab. Statist., 6 (2002), $147-155$.

[177] A. F. Ramirez, S. R. S. Varadhan, "Relative entropy and mixing properties of interacting particle systems", J. Math. Kyoto Univ., 36:4 (1996), 869-875.

[178] R. Holley, D. Stroock, "Diffusions on an infinite dimensional torus", J. Funct. Anal., 42:1 (1981), 29-63.

[179] D. Bakry, M. Ledoux, "Lévy-Gromov's isoperimetric inequality for an infinite-dimensional diffusion generator", Invent. Math., 123:2 (1996), 259-281.

[180] V. Liskevich, M. Röckner, "Strong uniqueness for certain infinite-dimensional Dirichlet operators and applications to stochastic quantization", Ann. Scuola Norm. Sup. Pisa Cl. Sci. (4), 27:1 (1998), 69-91; arXiv: math/9801144, 1998.

[181] J. M. A. M. van Neerven, "Second quantization and the $L^{p}$-spectrum of nonsymmetric Ornstein-Uhlenbeck operators", Infin. Dimens. Anal. Quantum Probab. Relat. Top., 8:3 (2005), 473-495.

В. И. Богачев (V.I. Bogachev)

Московский государственный университет

Поступила в редакцию

им. М. В. Ломоносова

E-mail: vibogach@mail.ru

Н. В. Крылов (N. V. Krylov)

University of Minnesota, Minneapolis, USA

E-mail: krylov@math.umn.edu

M. Pёкнер (M. Röckner)

Bielefeld University, Germany

E-mail: roeckner@math.uni-bielefeld.de 